\title{
Os exemplos de Superfícies Mínimas
} de Chen-Gackstätter

\author{
José Antenia Moraes Vilhena
}

\author{
DISSERTAÇÃO APRESENTADA \\ $\mathrm{AO}$ \\ INSTITUTO DE MATEMÁTICA E ESTATÍSTICA \\ DA \\ UNIVERSIDADE DE SÃO PAULO \\ PARA \\ OBTENÇÃO DO GRAU DE MESTRE \\ EM \\ MATEMÁTICA
}

\section{Área de Concentração: Geometria Diferencial Orientador: Prof. Dr. Plínio Amarante Quirino Simões}

Durante a elaboração deste trabalho o autor recebeu apoio financeiro do CNPq 


\title{
Os exemplos de Superfícies Mínimas de Chen-Gackstätter
}

\author{
Este exemplar corresponde à redação \\ final da dissertação devidamente \\ corrigida e defendida por \\ José Antonio Moraes Vilhena \\ e aprovada pela comissão julgadora.
}

São Paulo, maio de 1999.

Banca examinadora:

- Prof. Dr. Plínio Amarante Quirino Simões (Orientador) - IME-USP

- Prof. Dra. Maria Elisa Esteves Lopes Galvão - IME-USP

- Prof. Dr. Celso José da Costa - UFF 


\section{RESUMO}

Em 1981, Fritz Gackstätter e Chi Cheng Chen construíram duas superfícies mínimas completas orientadas em $\mathbb{R}^{3}$ do tipo Enneper, respectivamente, com curvatura total finita $-8 \pi$ e $-12 \pi$ e gênero 1 e 2 . Este trabalho, estuda a existência desses exemplos, assim como suas generalizações à uma família de superfícies mínimas completas do tipo Enneper em $\mathbb{R}^{3}$, respectivamente com curvatura total $-8 k \pi$ e $-12 k \pi$ e gênero $k$ e $2 k$, onde $k \in \mathbb{Z}_{+}$, obtidas em 1991 pelos matemáticos Hong Wang e Jianling Kang.

\footnotetext{
ABSTRACT

In 1981, Fritz Gackstätter and Chi Cheng Chen constructed two complete orientable minimal surfaces in $\mathbb{R}^{3}$, of Enneper's type, with finite total curvature $-8 \pi$ and $-12 \pi$ and genus 1 and 2 , respectively. This work is concerned with the existence of these examples, as well as their generalizations to a family of complete minimal surfaces of Enneper's type in $\mathbb{R}^{3}$, with total curvature $8 k \pi$ and $-12 k \pi$ and genus $k$ and $2 k$, respectively, where $k \in \mathbb{Z}_{+}$, obtained in 1991 by the mathematicians Hong Wang and Jianling Kang.
} 
Aos meus avós. 
Agradecimentos:

- Antes de tudo quero agradecer a Deus pela força espiritual.

- Aos meus avós, Manoel Olindo e Jovelina e meus pais, José e Benedita por terem me ajudado nesta caminhada.

- Ao Plínio pela excelente orientação.

- Aos professores Elza, Possani, Asperti, Célia, Maria Elisa, Verderesi, Rosa, Cláudia, Henrique, Cláudio pela amizade e ensinamentos matemáticos.

- Aos professores: José Miguel e Cleyton pelo apoio e incentivo.

- Ao: Brandemberg, Jorge, Silvano, Renato, Ermínio, João , João Batista, Adílson, Carlos, Ducival, Juacy e Márcio meus amigos e professores da UFPa.

- Aos colegas e amigos da UFPa: Lindomar, Izonete, Elielba, Sônia, Lília, Eucilene, Sandra, Cláudia, Ivan, Ulisses, Nonato, Washington, Larangeira, Antenor, Lia, Epaminondas, Benedito, Kátia, Albetã, Edvaldo, Renato, Érika, Ruth, Cláudio, Half, Adriana, Elena, Nelane e Amirando; pela grande amizade que temos.

- Aos meus irmãos: Maria José, José Luís, José Benedito, Maria Benedita, Maria de Jesus, Maria Lúcia, José Marcos, Maria Isabel, José Roberto, José Geraldo.

- Aos meus primos: Marlene, Socorro, Izonete, Carlos, Sérgio, Beto, Márcio e Sílvia.

- Aos meus tios, padrinhos e professores, João e Izolina.

- Aos meus tios e amigos do Miri: Lito, Marita, Raminho, Maroca, Benedito, Sílvia, Sidico, Maria, Maria José, Francisco, Guina, Bena, Dica, Cabral, Benedito, Sílvia.

- Aos amigos de Altamira: Júnior, Regilma, Judith, Alexandra, Marleide, Socorro, Cila, Íris, Patrícia, Claudomiro, João, Marizete, Sena, Marcos e Karina.

- Aos meus amigos do IME: Cecilia, Bárbara, Samuel, Major, Jorge, Regina, Raul, Pablo, Daniel, Liane, Adriana, Sidney, Marco Aurélio, Edson, Rolo, Caputi, Paulo, Ayumi, Marcela, Jair, Gláucio, Walter, Daniela, Clézio, Leandro, Alice, Sonti, Flávio, Jocirei, Ramiro, Ronaldo, Douglas, Kao, Maria, Walquíria, Cláudia, Raul, Xyoby, Antonio, Fernando, Daniela e Marcos. Pela amizade e pelo bom convívio.

- A los uruguayos: Eduardo, Arcadio, Perla, Nora, Jorge, Alicia, Silvia, Gustavo, Laura, Pablo, Leticia, Analia, Lucia, Alejandro, Nicolás.

- À Irene minha companheira de todos os momentos. Valeu. 


\section{Sumário}

Introdução

1

1 Resultados sobre Superfícies de Riemann, Funções Elípticas e Superfícies Mínimas 3

1.1 Superfícies de Riemann . . . . . . . . . . . . . . . . 3

1.1.1 Variedades Diferenciáveis. . . . . . . . . . . . . 3

1.1.2 Superfícies de Riemann Conformes . . . . . . . . . . 8

1.2 Construção de Funções Meromorfas sobre Superfícies de Riemann Compactas ............................. 9

1.3 Funçōes Algébricas. . . . . . . . . . . . . . . . . . . . . . . . 18

1.3.1 Superfícies de Riemann de Funções Algébricas . . . . . . . . 18

1.3.2 Alguns Exemplos de Superfícies de Funções Algébricas . . . . 21

1.3.3 Superfícies Hiperelípticas e Equações Algébricas . . . . . . . . 28

1.4 Funçòes Elípticas . . . . . . . . . . . . . . . . . . . . . . . . . . . . . 29

1.4.1 Definição da Função $\wp$-Weierstrass . . . . . . . . . . . . . . . 30

1.4 .2 Relação de Legendre . . . . . . . . . . . . . . . . . . . . . . 32

1.4.3 A Equação Diferencial da Função $\wp . . . . . .35$

1.4.4 A Aplicação Conforme pela Função $\wp . . . .36$

1.5 Superfícies Mínimas em $\mathbb{R}^{3}$. . . . . . . . . . . . . . . . . 40

1.5.1 Propriedades Locais . . . . . . . . . . . . . . . . 40

1.5.2 Resultados Globais sobre Superfícies Mínimas . . . . . . . . . 48 
2 Superfície Mínima de Chen-Gackstätter de Gênero 1

2.1 Fórmulas de $\wp$ e $\zeta$ no quadrado $[0,1,1+i, i] \ldots \ldots . \ldots . \ldots 5$

2.2 Exemplo de Chen-Gackstätter de gênero $1 \ldots \ldots . . \ldots 58$

3 Superfície Mínima de Chen-Gackstätter de Gênero 2

3.1 Adaptação à Linguagem Hiperelíptica do Exemplo de gênero 1 . . . 70

3.2 Exemplo de Chen-Gackstätter de gênero $2 \ldots \ldots . \ldots . . \ldots 72$

4 Generalização da Superfície de Chen-Gackstätter de Gênero $1 \quad 100$

5 Generalização da Superfície de Chen-Gackstätter de Gênero 2116

$\begin{array}{ll}\text { Referências Bibliográficas } & 134\end{array}$

$\begin{array}{lr}\text { Índice Remissivo } & 136\end{array}$ 


\section{INTRODUÇÃO}

Em 1981, os matemáticos C.C.Chen e F. Gackstätter construíram dois exemplos de superfícies mínimas completas de $\mathbb{R}^{3}$.

O primerio exemplo tem gênero 1 , curvatura total $-8 \pi$ e um único fim, e é construído usando, na representação de Weierstrass, a função $\wp$ de Weierstrass.

O segundo exemplo tem gênero 2 , curvatura total $-12 \pi$ e um único fim, e é construído usando na representação de Weierstrass uma função algébrica associada a uma superfície de Riemann hiperelíptica.

Estes exemplos foram os primeiros a serem obtidos explicitamente, especificandose propriedades geométricas, no caso as simetrias e o comportamento do fim do tipo apresentado pela superfície de Enneper.

O objetivo da dissertação é uma exposição da existência dos exemplos de C.C. Chen e F. Gackstätter, descritos acima, e suas generalizações obtidas pelos matemáticos, H. Wang e J. Kang em 1991, em [26], que consiste na construção de uma família de exemplos de superfícies mínimas completas do tipo Enneper, com gênero igual a $k$ e $2 k$, curvatura total, $-8 k \pi$ e $-12 k \pi$, e um único fim de ordem $2 k+1$, respectivamente.

No capítulo 1, apresentaremos resultados sobre superfícies mínimas, superfícies de Riemann hiperelípticas, funções algébricas, funções elípticas e superfícies mínimas em $\mathbb{R}^{3}$.

No capítulo 2 , estudaremos as funções, $\wp$ de Weierstrass e $\zeta$ de Weierstrass no caso em que o paralelogramo fundamental é o quadrado de vértices $[0,1,1+i, i]$ e daremos a demonstração do primeiro, dos quatro teoremas centrais deste trabalho, o qual consiste no primeiro exemplo de Chen-Gackstätter, cuja representação de Weierstrass é $g=\frac{A \wp^{\prime}}{\wp}$ e $\eta=2 \wp d z$.

No capítulo 3, faremos inicialmente uma adaptação à linguagem hiperelíptica da representação $g=\frac{A \wp^{\prime}}{\wp}$ e $\eta=2 \wp d z$ do capítulo 2 , e usando a relação entre a teoria de superfícies de Riemann e superfícies mínimas em $\mathbb{R}^{3}$, apresentaremos a demonstração da existência do segundo exemplo de Chen-Gackstätter. 
Os capítulo 4 e 5, estão direcionados para a demonstração da existência dos exemplos de H. Wang e J. Kang, o primeiro é obtido imergindo em $\mathbb{R}^{3}$ a superfície de Riemann compacta $\bar{R}_{k}$ de gênero $k$, associada a função algébrica

$$
w(z)=\left[z^{k}\left(z^{2}-a^{2}\right)\right]^{\frac{1}{k+1}},
$$

menos um ponto conveniente, e o segundo exemplo é obtido imergindo a superfície de Riemann compacta $\bar{R}_{2 k}$, da equação algébrica

$$
w(z)=\left[z\left(z^{2}-a^{2}\right)^{k}\left(z^{2}-b^{2}\right)\right]^{\frac{1}{k+1}},
$$

menos um ponto de ramificação correspondente ao infinito. 


\section{Capítulo 1}

\section{Resultados sobre Superfícies de Riemann, Funções Elípticas e Superfícies Mínimas}

Neste capítulo exporemos alguns resultados sobre superfícies de Riemann, funções elípticas e superfícies mínimas em $\mathbb{R}^{3}$.

\subsection{Superfícies de Riemann}

\subsubsection{Variedades Diferenciáveis}

Definição 1.1.1 Uma variedade diferenciável de dimensão 2 , de classe $C^{\infty}$, é um espaço topológico $M$ de Hausdorff com base enumerável, munido de uma família

$$
\mathcal{F}(M):=\left\{\left(U_{\alpha}, V_{\alpha}, z_{\alpha}\right), \alpha \in I\right\}
$$

de homeomorfismos $z_{\alpha}: U_{\alpha} \longrightarrow V_{\alpha}$, onde $U_{\alpha}$ é um aberto de $M$ e $V_{\alpha}$ aberto de $\mathbb{C}$, tal que:

(i) $\bigcup_{\alpha \in I} U_{\alpha}=M$ 
(ii) Se $\left(U_{\alpha}, V_{\alpha}, z_{\alpha}\right),\left(U_{\beta}, V_{\beta}, z_{\beta}\right) \in \mathcal{F}(M)$ são tais que $U_{\alpha} \cap U_{\beta} \neq \emptyset$, para todo $\alpha, \beta \in I$, então $z_{\beta} \circ z_{\alpha}^{-1}$ e $z_{\alpha} \circ z_{\beta}^{-1}$ são difeomorfismos de classe $C^{\infty}$.

(iii) Seja $(U, V, z)$, onde $U$ é um aberto de $M, V$ é um aberto de $\mathbb{C}, z: U \longrightarrow V$ um homeomorfismo entre $U$ e $V, \quad e\left(U_{\alpha}, V_{\alpha}, z_{\alpha}\right) \in \mathcal{F}(M)$ é tal que $U \cap U_{\alpha} \neq \emptyset$, $z \circ z_{\alpha}^{-1} \quad$ e $z_{\alpha} \circ z^{-1}$ são difeomorfismos $C^{\infty}$, então $(U, V, z) \in \mathcal{F}(M)$.

\section{Observação:}

(1) $\mathcal{F}(M)$ é a estrutura diferenciável da variedade $M$.

(2) Os elementos $\left(U_{\alpha}, V_{\alpha}, z_{\alpha}\right) \in \mathcal{F}(M)$ são coordenadas locais de $M$, às vezes denotaremos apenas por $z_{\alpha}$.

Um atlas sobre $M$ é uma família de coordenadas locais, $\mathcal{F}_{0}=\left\{\left(U_{\alpha}, V_{\alpha}, z_{\alpha}\right) ; \alpha \in I\right\}$ satisfazendo as propriedades $(i)$ e $($ ii $)$, que induz uma estrutura diferenciável $\mathcal{F}(M)$ contendo $\mathcal{F}_{0}$, dada por:

$\mathcal{F}(M):=\left\{(U, V, z) ; z \circ z_{\alpha}^{-1}\right.$ e $z_{\alpha} \circ z^{-1}$ são difeomorfismos $\left.C^{\infty},\left(U_{\alpha}, V_{\alpha}, z_{\alpha}\right) \in \mathcal{F}_{0}\right\}$

Definição 1.1.2 Uma superfície de Riemann $M$ é uma variedade de dimensão 2 conexa, com uma família máxima $\mathcal{H}(M) \subset \mathcal{F}(M)$ de homeomorfismos, onde as mudanças de coordenadas são funções holomorfas. $\mathcal{H}(M)$ é a estrutura holomorfa da superfície de Riemann.

Observação: Uma superfície de Riemann é um par $(M, \mathcal{H}(M))$, usualmente denotaremos por $M$.

Seja $\mathcal{H}_{0}$ um atlas holomorfo sobre $M$, isto é, uma família de coordenadas locais tais que cobrem $M$ e a mudança de coordenada é uma função holomorfa. Então, $\mathcal{H}_{0}$ induz uma estrutura holomorfa sobre $M$ contendo $\mathcal{H}_{0}$,

$$
\mathcal{H}(M):=\left\{(U, V, z) ; z \circ z_{\alpha}^{-1} \text { e } z_{\alpha} \circ z^{-1} \text { são holomorfas }, \forall\left(U_{\alpha}, V_{\alpha}, z_{\alpha}\right) \in \mathcal{H}_{0}\right\}
$$

Assim, $(M, \mathcal{H}(M))$ é uma superfície de Riemann.

Exemplos: 
(1) O plano complexo $\mathbb{C}:=\{z=u+i v ; u, v \in \mathbb{R}\}$.

$$
\mathcal{H}_{0}:=\{(\mathbb{C}, \mathbb{C}, i d) ; \text { id }: \mathbb{C} \longrightarrow \mathbb{C} \text { é a função identidade }\}
$$

é um atlas holomorfo sobre $\mathbb{C}$, que induz uma estrutura holomorfa que torna $\mathbb{C}$ uma superfície de Riemann. É o plano complexo.

(2) A esfera de Riemann $\mathbb{C} \cup\{\infty\}$. Sobre a esfera unitária,

$$
\begin{gathered}
S^{2}(1):=\left\{(u, v, t) \in \mathbb{R}^{3} ; u^{2}+v^{2}+t^{2}=1\right\} \text { em } \mathbb{R}^{3}, \\
\mathcal{H}_{0}:=\left\{\left(S^{2}(1) \backslash\{(0,0,1)\}, \mathbb{C}, \pi_{1}\right),\left(S^{2}(1) \backslash\{(0,0,-1)\},(\mathbb{C} \backslash\{0\}) \cup\{\infty\}, \psi \circ \pi_{2}\right)\right\},
\end{gathered}
$$

É um atlas holomorfo, que induz uma estrutura holomorfa que torna $S^{2}(1)$ uma superfície de Riemann indicada por $\mathbb{C} \cup\{\infty\}$. É a esfera de Riemann.

onde, $\quad \pi_{1}(u, v, t)=\frac{u+i v}{1-t}, \pi_{2}(u, v, t)=\frac{u+i v}{1+t} \quad$ e $\quad \psi(z):=\bar{z}, z=u+i v$

Donde segue,

$\pi_{1}^{-1}(z)=\left(\frac{2 u}{|z|^{2}+1}, \frac{2 v}{|z|^{2}+1}, \frac{|z|^{2}-1}{|z|^{2}+1}\right)$ e $\left(\psi \circ \pi_{2}\right)^{-1}(z)=\left(\frac{2 u}{|z|^{2}+1}, \frac{-2 v}{|z|^{2}+1}, \frac{1-|z|^{2}}{|z|^{2}+1}\right)$.

Logo, as mudanças de coordenadas são:

$$
\begin{aligned}
\left(\psi \circ \pi_{2}\right) \circ \pi_{1}^{-1}, \pi_{1} \circ\left(\psi \circ \pi_{2}\right)^{-1}: \mathbb{C} \backslash\{0\} & \longrightarrow \mathbb{C} \backslash\{0\} \\
z & \longmapsto \frac{1}{z}
\end{aligned}
$$

O terceiro exemplo que faremos de superfície de Riemann são os toros $T_{\left(w_{1}, w_{2}\right)}$. Para a descrição desses exemplos precisamos de algumas definições:

\section{Definição 1.1.3}

(i) O reticulado em $\mathbb{C}$ com geradores $w_{1}$ e $w_{2}$, é o conjunto:

$$
L=L\left(w_{1}, w_{2}\right):=\left\{w=m w_{1}+n w_{2} ; m, n \in \mathbb{Z} e \operatorname{Im}\left(\frac{w_{2}}{w_{1}}\right)>0\right\}
$$




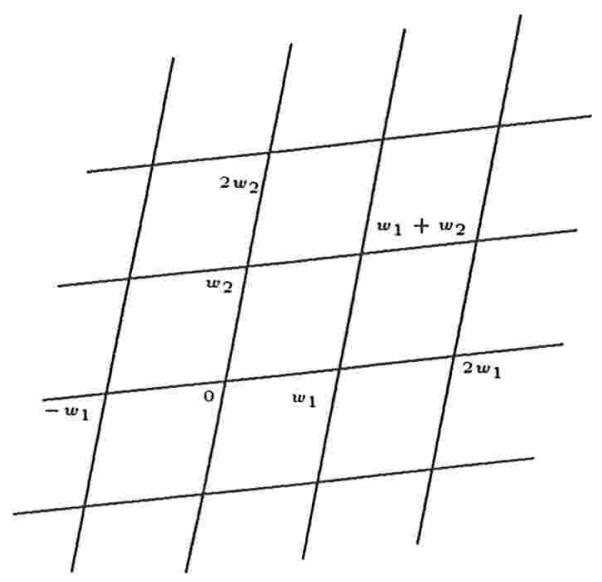

Figura 1.1

(ii) Dados dois números complexos $z, z^{\prime}$, dizemos que $z$ é L-equivalente a $z^{\prime}$ se existem inteiros $m, n$ tais que $z-z^{\prime}=m w_{1}+n w_{2} \in L\left(w_{1}, w_{2}\right)$. Sejam ainda $[z]=\left\{z^{\prime}: z-z^{\prime} \in L\right\}$ a classe de equivalência que tem como representante o $z, e$ $\mathbb{C} / L$ o conjunto de todas as classes de equivalência.

(iii) Dado $z_{0} \in L\left(w_{1}, w_{2}\right)$, definimos o conjunto

$$
F=F\left(z_{0}\right):=\left\{z=z_{0}+\alpha w_{1}+\beta w_{2} ; 0 \leq \alpha, \beta<1\right\},
$$

como na figura 1.2 .

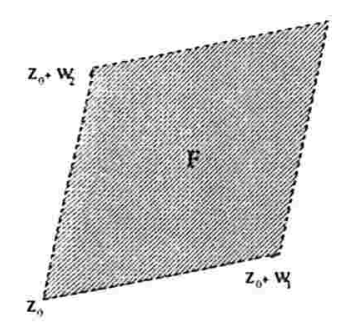

Figura 1.2

Atribuindo a $\mathbb{C} / L$, a topologia quociente, $\mathbb{C} / L$ é de Hausdorff, tem base enumerável e a projeção canônica:

$$
\begin{aligned}
& \pi: \mathbb{C} \longrightarrow \mathbb{C} / L \\
& z \longmapsto[z]
\end{aligned}
$$


é contínua e aberta. Além disso, se $V$ é um subconjunto aberto de $\mathbb{C}$ que não tem dois pontos equivalentes sobre $L$, então o atlas $\mathcal{H}_{0}=\left\{\left(\pi(V), V,\left(\left.\pi\right|_{\pi(V)}\right)^{-1}\right)\right\}$ induz uma estrutura holomorfa $\mathcal{H}(\mathbb{C} / L)$ contendo $\mathcal{H}_{0}$, que torna $\mathbb{C} / L$ uma superfície de Riemann, que denotaremos por $T_{\left(w_{1}, w_{2}\right)}$.

Consideremos agora o homeomorfismo,

$$
\begin{gathered}
\varphi: \quad F(0) \subset \mathbb{C} \longrightarrow S^{1} \times S^{1} \\
z \longmapsto\left(e^{2 \pi i \alpha}, e^{2 \pi i \beta}\right)
\end{gathered}
$$

onde $z=\alpha w_{1}+\beta w_{2}, 0 \leq \alpha, \beta<1$ e $S^{1}=\{z \in \mathbb{C} ;|z|=1\}$. Logo, a aplicação $\psi$ dada no diagrama abaixo é um homeomorfismo.

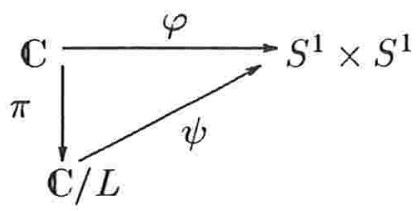

Portanto, se $z \in F(0)$ temos que $\mathbb{C} / L$ é homeomorfo ao toro $S^{1} \times S^{1}$.

Sejam agora $L=L\left(w_{1}, w_{2}\right)$ e $L^{\prime}=L^{\prime}\left(w_{1}^{\prime}, w_{2}^{\prime}\right)$ reticulados de $\mathbb{C}$ e as superfícies de Riemann compactas de gênero $1, T_{\left(w_{1}, w_{2}\right)}=\mathbb{C} / L$ e $T_{\left(w_{1}, w_{2}\right)}^{\prime}=\mathbb{C} / L^{\prime}$ como no exemplo 3 acima. Dizemos que as estruturas complexas de $T_{\left(w_{1}, w_{2}\right)}$ e $T_{\left(w_{1}, w_{2}\right)}^{\prime}$ são equivalentes se existir um difeomorfismo holomorfo, isto é um biholomorfismo $f: T \longrightarrow T^{\prime}$. O teorema enunciado abaixo descreve todas as estruturas complexas de gênero 1 .

Teorema 1.1.4 Seja $F M \subset \mathbb{C}$ o conjunto figura modular, definido por:

$$
F M=\{\tau \in \mathbb{C}:|\tau| \geq 1,|\operatorname{Re}\{\tau\}| \leq 1 / 2 \text { e } \operatorname{Im}\{\tau\}>0\}
$$

\section{Então,}

i) Se $M$ é uma superfície de Riemann compacta de gênero 1, existe $\tau \in F M$ tal que $M$ é conformemente equivalente a $\mathbb{C} / L(1, \tau)$;

ii) Se $\tau, \tau^{\prime} \in F M$ e $\mathbb{C} / L(1, \tau)$ é conformemente equivalente a $\mathbb{C} /\left(1, \tau^{\prime}\right)$ temos que $\tau=\tau^{\prime}$ ou $\tau, \tau^{\prime} \in \partial F M$ e $\tau^{\prime}=-\bar{\tau}$. 
Demonstração: ver [4]

Observação: A condição (ii) do teorema acima às vezes aparece na literatura, como: $\mathbb{C} / L(1, \tau)$ é conformemente equivalente a $\mathbb{C} /\left(1, \tau^{\prime}\right)$ se,

$$
\tau=\frac{a \tau^{\prime}+b}{c \tau^{\prime}+d}, \text { onde } a, b, c, d \text { são inteiros tais que } a d-b c= \pm 1 .
$$

\subsubsection{Superfícies de Riemann Conformes}

Definição 1.1.5 Uma função contínua $f: M \longrightarrow N$ entre duas superfícies de Riemann é holomorfa se para qualquer coordenada local $(U, z(U), z) \in \mathcal{H}(M)$ e $(V, \zeta(V), \zeta) \in \mathcal{H}(N)$, respectivamente de $M$ e $N$, tal que $U \cap f^{-1}(V) \neq \emptyset$, a função

$$
\zeta \circ f \circ z^{-1}: z\left(U \cap f^{-1}(V)\right) \longrightarrow \zeta(V)
$$

é holomorfa. Além disso se $f$ é bijetora, então $M$ é conformemente equivalente (ou biholomorfa) à $N$.

Teorema 1.1.6 Sejam $M$ e $N$ superfícies de Riemann compactas e $f: M \longrightarrow N$ uma função holomorfa não constante. Então fé sobrejetora.

Demonstração: Como $f(M)$ é um subconjunto aberto e fechado de $N$ e $N$ conexo, então $f(M)=N$.

Seja $f: M \longrightarrow N$ uma função holomorfa não constante entre as superfícies de Riemann $M$ e $N$. Escolhendo convenientemente as coordenadas locais $(\tilde{U}, \tilde{V}, \tilde{z})$ de $M$ se anulando em $P \in \tilde{U}$ e $(U, V, \zeta)$ de $N$ se anulando em $f(P) \in U, \operatorname{com} \tilde{U}, U$ simplesmente conexos,$f$ é localmente dada por

$$
\begin{aligned}
\zeta=f(\tilde{z}) & =\tilde{z}^{n}\left(a_{0}+a_{1} \tilde{z}+\ldots\right), \quad n>0, \quad a_{0} \neq 0 \\
& =\tilde{z}^{n} g(\tilde{z}),
\end{aligned}
$$


onde $g(\tilde{z})$ é analítica e $g(0) \neq 0$. Logo, existe uma função analítica $h(\tilde{z})$ ( em um disco $D \subset \tilde{V})$ tal que $g(\tilde{z})=[h(\tilde{z})]^{n}$. Então $z=\tilde{z} h(\tilde{z})$ ainda é coordenada local que se anula em $P$. Em termos desta coordenada $f$ é dada por,

$$
\zeta=z^{n}
$$

Como esse $n$ independe da particular coordenada local, dizemos que $f$ tem multiplicidade $n$ em $P \in M$, ou que $f$ assume o valor $f(P) n$ vezes em $P$. O ponto $P$ é chamado ponto de ramificação de $f$ se $n>1$ e o número $n-1$ será chamado de ordem de ramificação de $f$ em $P$.

Proposição 1.1.7 Seja $f: M \longrightarrow N$ uma função holomorfa não constante entre superfícies de Riemann compactas. Existe um inteiro positivo $m$ tal que qualquer $Q \in N$ é assumido precisamente $m$ vezes em $M$ por $f$, levando-se em conta as multiplicidades; isto é, para todo $Q \in N$,

$$
\sum_{P_{i} \in f^{-1}(Q)} n_{i}=m, \quad \text { onde } n_{i} \text { é a multiplicidade de } f \text { em } P_{i} \text {. }
$$

Demonstração: ver [7] pag. 12.

Corolário 1.1.8 Se $M$ é uma superfície de Riemann compacta ef $: M \longrightarrow \mathbb{C} \cup\{\infty\}$ é holomorfa, então, levando-se em conta as multiplicidades, todo $Q \in \mathbb{C} \cup\{\infty\}$ é assumido um número fixo $m$ de vezes.

Definição 1.1.9 O número $m$ dado em 1.2, é o grau de $f$, e é denotado por grau $(f)$.

\subsection{Construção de Funções Meromorfas sobre Su- perfícies de Riemann Compactas}

O que podemos dizer a respeito das funções meromorfas sobre uma superfície compacta $M$ de gênero $p$ com polos somente em um ponto?. Se existe essa função 
qual a ordem do polo?. Podemos responder estas perguntas aplicando o teorema de Riemann-Roch, o qual relaciona a dimensão de determinados espaços vetoriais de funções meromorfas e diferenciais abelianas, a ordem da função meromorfa sobre $M$ e o gênero $p$ de $M$. Veremos também que existe um número finito de pontos $P_{i} \in M$ (chamados pontos de Weierstrass) para os quais existe sobre $M$ uma função meromorfa $f$, holomorfa em $M \backslash\left\{P_{i}\right\}$, com polo em $P_{i}$ de ordem no máximo igual a $p$.

Agora daremos algumas definições prévias. Primeiro definiremos 0 -formas e 1-formas sobre superfícies de Riemann:

Definição 1.2.1 Uma 0-forma em uma superfície de Riemann $M$ é uma função diferenciável sobre $M$. Uma 1-forma diferenciável $\omega \in \Lambda^{1}(M)$ (espaço vetorial das 1-formas) é uma associação ordenada de duas funções contínuas $f$ e g para cada coordenada local $\left(U_{1}, V_{1}, z=x+i y\right)$ sobre $M$ tal que

$$
\omega:=f d x+g d y
$$

é invariante por mudança de coordenadas, isto é, se $\left(U_{2}, V_{2}, \tilde{z}=\tilde{x}+i \tilde{y}\right)$ é outra coordenada local sobre $M$ e $U_{1} \cap U_{2} \neq \emptyset$, e se $\omega$ associa as funções $\tilde{f}, \tilde{g}$ à $\tilde{z}$ então,

$$
\left(\begin{array}{l}
\tilde{f}(\tilde{z}) \\
\tilde{g}(\tilde{z})
\end{array}\right)=\left(\begin{array}{ll}
\frac{\partial x}{\partial \tilde{x}} & \frac{\partial y}{\partial \tilde{x}} \\
\frac{\partial x}{\partial \tilde{y}} & \frac{\partial y}{\partial \tilde{y}}
\end{array}\right)\left(\begin{array}{c}
f(z(\tilde{z})) \\
g(z(\tilde{z}))
\end{array}\right)
$$

As 1-formas meromorfas são chamadas de diferenciais abelianas. As diferenciais abelianas que são holomorfas são as de primeira espécie; enquanto que as diferenciais abelianas meromorfas com resíduo zero são as de segunda espécie. Finalmente uma diferencial abeliana geral, a qual pode ter resíduo não nulo será chamada de terceira espécie.

Proposição 1.2.2 Sobre uma superfície de Riemann compacta $M$ de gênero $p$, o espaço vetorial das diferenciais holomorfas tem dimensão $p$.

Demonstração: ver [24] 
Definição 1.2.3 Um divisor sobre $M$ é um símbolo formal $D=P_{1}{ }^{\mu_{1}} P_{2}{ }^{\mu_{2}} \ldots P_{m}{ }^{\mu_{m}}$, $\operatorname{com} P_{i} \in M, \mu_{i} \in \mathbb{Z}$.

$O$ inteiro $\mu_{k}$ é chamado ordem de $P_{k}$, e o grau de $D$, denotado por gr $[D]$ é a soma das ordens de $D$,

$$
\operatorname{gr}[D]=\sum_{k=1}^{m} \mu_{k}
$$

Se $D_{1}=P_{1}{ }^{\mu_{1}} P_{2}^{\mu_{2}} \ldots P_{m}^{\mu_{m}}$ e $D_{2}=Q_{1}{ }^{\nu_{1}} Q_{2}{ }^{\nu_{2}} \ldots Q_{n}{ }^{\nu_{n}}$,

$$
D_{1} D_{2}=P_{1}^{\mu_{1}} P_{2}^{\mu_{2}} \ldots P_{m}^{\mu_{m}} Q_{1}^{\nu_{1}} Q_{2}{ }^{\nu_{2}} \ldots Q_{n}{ }^{\nu_{n}}
$$

onde $P_{i}^{\mu_{i}} Q_{j}{ }^{\nu_{\jmath}}=Q_{j}{ }^{\nu_{\jmath}} P_{i}^{\mu_{i}}, \quad P_{i}^{\mu_{i}} P_{i}^{\nu_{i}}=P_{i}^{\mu_{i}+\nu_{i}}$ é o produto de $D_{1}$ por $D_{2}$ e

$$
D_{2}^{-1}=\frac{1}{D_{2}}=Q_{1}^{-\nu_{1}} Q_{2}^{-\nu_{2}} \ldots Q_{n}^{-\nu_{n}}
$$

é o inverso de $D_{2}$. Logo,

$$
\operatorname{gr}\left[D_{1} D_{2}^{-1}\right]=\sum_{k=1}^{m} \mu_{k}-\sum_{j=1}^{n} \nu_{j}=\operatorname{gr}\left[D_{1}\right]-\operatorname{gr}\left[D_{2}\right]
$$

O divisor $D=P_{1}{ }^{\mu_{1}} P_{2}{ }^{\mu_{2}} \ldots P_{m}{ }^{\mu_{m}}$ é inteiro se $\mu_{k} \geq 0 \quad \forall k=1,2, \ldots, m$. Se $D_{1} D_{2}^{-1}$ é inteiro, dizemos que $D_{2}$ divide $D_{1}$ ou que $D_{1}$ é múltiplo de $D_{2}$.

Se $f \not \equiv 0$ é uma função meromorfa sobre $M$, então a ordem de $f$ no ponto $P_{i}$, em termos de uma coordenada local $z_{\alpha}$, tal que $z_{\alpha}\left(P_{i}\right)=0$, é dada por :

$$
\operatorname{ord}_{P_{i}}[f]:=\left\{\begin{array}{cl}
n, & \text { se } f \text { tem um zero de ordem } n \text { em } P_{i} \\
0, & \text { se } f \text { é regular e não nula em } P_{i} \\
-m, & \text { se } f \text { tem um polo de ordem } m \text { em } P_{i}
\end{array}\right.
$$

E se $\eta$ é uma diferencial abeliana, dada localmente por $\eta=f d z_{\alpha}$, então a ordem de $\eta$ no ponto $P_{i}$ é igual a ordem de $f$ em $P_{i}$.

Definição 1.2.4 Se $f$ é uma função meromorfa ( $f \not \equiv 0$ ) sobre $M$ e $\eta$ é uma diferencial abeliana sobre $M$, o divisor $(f)$ de $f$ é dado por:

$$
(f)=\prod_{P \in M} P^{\operatorname{ord} P[f]}
$$


e o divisor $(\eta)$ de $\eta$ por:

$$
\left.(\eta)=\prod_{P \in M} P^{o r d} d_{P} \eta\right]
$$

Dizemos que $f, \eta$ são múltiplos de um divisor $D$, se $D$ divide, respectivamente $(f)$ ou $(\eta)$. Isto significa que se

$$
D=\frac{1}{P_{1}^{\mu_{1}} P_{2}^{\mu_{2}} \ldots P_{m}{ }^{\mu_{m}}}, \quad \mu_{i} \geq 0
$$

a função $f$ ou a diferencial $\eta=f(z) d z$, têm respectivamente polos de ordem no máximo $\mu_{i}$ em $P_{i}$.

$\mathrm{Se}$

$$
D=\frac{P_{1}^{\mu_{1}} P_{2}^{\mu_{2}} \ldots P_{m}^{\mu_{m}}}{Q_{1}^{\nu_{1}} Q_{2}{ }^{\nu_{2}} \ldots Q_{n}^{\nu_{n}}}, \quad \mu_{i} \geq 0, \nu_{j} \geq 0
$$

$f$ e $\eta$ têm respectivamente polos de ordem no máximo $\nu_{i}$ em $Q_{i}$ e têm zeros de ordem no mínimo $\mu_{j}$ em $P_{j}$.

É interessante observar que para qualquer função meromorfa $f$ sobre uma superfície de Riemann compacta, o número de zeros é igual ao número dos polos, levando-se em conta as multiplicidades. Portanto, $\operatorname{gr}[(f)]=0$.

Observe que nem todos os divisores são divisores de funções meromorfas. O divisor é principal se ele é um divisor de uma função meromorfa.

$\mathrm{O}$ conjunto de todos os divisores formam um grupo comutativo em relação ao produto 1.3. Se $D_{1}=(f)$ e $D_{2}=(g)$, então $D_{1}^{-1}=\left(\frac{1}{f}\right)$ e $D_{1} D_{2}=(f g)$, de modo que os divisores principais formam um subgrupo do grupo de todos os divisores. Podemos formar o grupo quociente de todos os divisores sobre o subgrupo dos divisores principais. Cada elemento do grupo quociente é uma classe de divisores. $D_{1}$ e $D_{2}$ pertencem a mesma classe $\left(D_{1} \sim D_{2}\right)$ se, e somente se $D_{1} D_{2}^{-1}=(f)$, onde $f$ é uma função meromorfa sobre $M$, e então $\operatorname{gr}\left[D_{1}\right]=\operatorname{gr}\left[D_{2}\right]$.

Se $\eta_{1}$ e $\eta_{2}$ são duas diferenciais abelianas sobre $M, \frac{\eta_{1}}{\eta_{2}}$ é uma função meromorfa sobre $M, \operatorname{logo}\left(\eta_{1}\right) \sim\left(\eta_{2}\right)$. Assim os divisores de todas as diferenciais estão na mesma classe.

Dado o divisor $D=P_{1}{ }^{\mu_{1}} P_{2}{ }^{\mu_{2}} \ldots P_{m}{ }^{\mu_{m}}$. Sejam:

$$
L[D]:=\left\{f ; f \text { é meromorfa, } \quad \operatorname{ord}_{P_{i}}[f] \geq \mu_{i}, \forall i=1, \ldots, m\right\}
$$


o espaço vetorial das funções meromorfas sobre $M$ que são múltiplas de $D$, e

$$
\Omega[D]:=\left\{\eta ; \eta \text { diferencial abeliana, } \operatorname{ord}_{P_{\mathrm{i}}}[\eta] \geq \mu_{i}, \forall i=1, \ldots, m\right\},
$$

o espaço vetorial de todas as diferenciais abelianas $\eta$, cujos divisores $(\eta)$, são múltiplos de $D$. Denotaremos suas dimensões, respectivamente, por $r[D]$ e $i[D]$.

Lema 1.2.5 Os espaços vetoriais $L[D]$ e $\Omega[D]$ só dependem da classe dos divisores, isto é,

$$
D_{1} \sim D_{2} \Rightarrow\left\{\begin{array}{lll}
L\left[D_{1}\right] & \text { é isomorfo a } & L\left[D_{2}\right] \\
\Omega\left[D_{1}\right] & \text { é isomorfo a } & \Omega\left[D_{2}\right]
\end{array}\right.
$$

Demonstração: Como $\frac{D_{1}}{D_{2}}=(f)$, para alguma função meromorfa $f$. As aplicações:

$$
\begin{aligned}
\mathrm{L}\left[D_{2}\right] & \longrightarrow L\left[D_{1}\right] & \Omega\left[D_{2}\right] & \longrightarrow \Omega\left[D_{1}\right] \\
h & \longmapsto h f & \omega & \longmapsto \omega f
\end{aligned}
$$

são isomorfismos.

Proposição 1.2.6 Se $\eta$ é qualquer diferencial abeliana, $\eta \not \equiv 0$, então $i[D]=r\left[\frac{D}{(\eta)}\right]$, para qualquer divisor $D$.

Demonstração: Seja $\omega \in \Omega[D]$, então $(\omega)$ é múltiplo de $D$, ou seja $\frac{(\omega)}{D}$ é inteiro. Como, $\left(\frac{\omega}{\eta}\right)=\frac{(\omega)}{D} \frac{D}{(\eta)},\left(\frac{\omega}{\eta}\right)$ é múltiplo de $\frac{D}{(\eta)}, \log o \frac{\omega}{\eta} \in L\left[\frac{D}{(\eta)}\right]$. A aplicação,

$$
\begin{aligned}
\Omega[D] & \longrightarrow L\left[\frac{D}{(\eta)}\right] \\
\omega & \longmapsto \frac{\omega}{\eta}
\end{aligned}
$$

é um isomorfismo. De fato ela é linear. Injetora, pois de $\frac{\omega}{\eta} \equiv 0$, temos que $\omega \equiv 0$ e é sobrejetora já que $f \in L\left[\frac{D}{(\eta)}\right]$ é imagem de $f \eta \in \Omega[D]$. Portanto, $i[D]=r\left[\frac{D}{(\eta)}\right]$.

Teorema 1.2.7 (Riemann-Roch) Seja $M$ uma superfície de Riemann compacta de gênero p. Então:

$$
r\left[\frac{1}{D}\right]=\operatorname{gr}[D]+i[D]-p+1
$$


Demonstração: ver [24]

Definição 1.2.8 Seja $D$ um dividor inteiro, chamamos de inequação de Riemann a desigualdade

$$
r\left[\frac{1}{D}\right] \geq g r[D]-p+1
$$

Esta desigualdade significa que o número de funções meromorfas linearmente independentes com polos de ordem no máximo $\mu_{k}$ nos $m$ pontos $P_{k}, \quad k=1, \ldots, m$, é no mínimo $\sum_{k=1}^{m} \mu_{k}-p+1$. Se vale a igualdade, $i[D]=0$; logo não existem diferenciais abelianas não nulas cujos divisores são múltiplos de $D$.

Corolário 1.2.9 Para qualquer diferencial abeliana $\eta$, sobre uma superfície de Riemann compacta de gênero $p$ vale a fórmula $g r[(\eta)]=2 p-2$.

Demonstração: Pondo $D=(\eta)$ na fórmula 1.4 temos,

$$
r\left[\frac{1}{(\eta)}\right]=\operatorname{gr}[(\eta)]+i[(\eta)]-p+1
$$

Por outro lado, indicando-se por 1 a unidade do grupo dos divisores, segue da proposição 1.2.6, que $i[(\eta)]=r[1]$ e $r\left[\frac{1}{(\eta)}\right]=i[1]$. Como $r[1]=1$, temos $i[(\eta)]=1$. Por último usando a proposição 1.2 .2 , temos $i[1]=p$, logo $r\left[\frac{1}{(\eta)}\right]=p$. Portanto,

$$
p=\operatorname{gr}[(\eta)]-p+2 \Rightarrow \operatorname{gr}[(\eta)]=2 p-2
$$

Veremos agora outras consequências imediatas do teorema de Riemann-Roch.

Quando $p=1$, para toda diferencial abeliana $\eta$, temos $\operatorname{gr}[(\eta)]=2.1-2=0$. Portanto, para qualquer diferencial abeliana sobre um toro o número de zeros é igual ao número de polos. Em particular como uma diferencial de primeira espécie não tem polos ela também não tem zeros, isto é, $i[P]=0$. Então, aplicando o teorema de Riemann-Roch, temos

$$
r\left[\frac{1}{P}\right]=\operatorname{gr}[P]+i[P]-p+1=1+0-1+1=1
$$


Como $r\left[\frac{1}{P}\right]=1$, então as funções constantes formam uma base para $L\left[\frac{1}{P}\right]$. Em outras palavras, uma função meromorfa não pode ter um polo simples sobre uma superfície compacta de gênero 1 .

Corolário 1.2.10 Se $\operatorname{gr}[D]<0$, então $r\left[\frac{1}{D}\right]=0$.

\section{Demonstração:}

Se não, existiria $f \in L\left[\frac{1}{D}\right]$, a qual seria uma função meromorfa com mais polos do que zeros. pois $\operatorname{gr}\left[\frac{1}{D}\right]=-\operatorname{gr}[D]>0$. Isto contradiz o resultado que se " $f$ é uma função meromorfa sobre uma superfície de Riemann compacta, então a soma das ordens dos zeros é igual a soma das ordens dos polos". Isto é, dimensão de $L\left[\frac{1}{D}\right]$ é zero, $r\left[\frac{1}{D}\right]=0$.

Corolário 1.2.11 Se $\operatorname{gr}[D]>2 p-2$, então $i[D]=0$.

\section{Demonstração:}

De fato, pela proposição $(1.2 .6), \quad i[D]=r\left[\frac{D}{(\eta)}\right]$, onde $\eta$ é uma diferencial abeliana. Como $g r[(\eta)]=2 p-2$ pelo corolário 1.2 .9 , temos

$$
\operatorname{gr}\left[\frac{(\eta)}{D}\right]=\operatorname{gr}[(\eta)]-\operatorname{gr}[D]=(2 p-2)-\operatorname{gr}[D]<0 .
$$

Pelo corolário (1.2.10), segue que $r\left[\left(\frac{(\eta)}{D}\right)^{-1}\right]=r\left[\frac{D}{(\eta)}\right]=0$, e portanto $i[D]=0$.

Corolário 1.2.12 Uma diferencial holomorfa (de primeira espécie) $\eta$ tem $2 p-2$ zeros sobre uma superfície de Riemann compacta $M$ de gênero $p$.

\section{Demonstração:}

É imediato, pois $\eta$ não tem polos sobre $M$ e do corolário $1.2 .9, g r[(\eta)]=2 p-2=$ $\mathcal{Z}-\mathcal{P}$, onde $\mathcal{Z}$ é o número de zeros e $\mathcal{P}$ é o número de polos. Logo $\mathcal{Z}=2 p-2$.

Teorema 1.2.13 Sejam $M$ uma superfície de Riemann compacta de gênero $p$, e a função holomorfa $f: M \longrightarrow \mathbb{C} \cup\{\infty\}$ com grau $n$, tendo $P_{1}, P_{2}, \ldots, P_{N}$ como 
pontos de ramificação, com respectivas multiplicidades $r_{i}$ (isto é, $f$ em $P_{i}$ tem ordem de ramificação $\left.r_{i}-1\right)$. Então:

$$
V=\sum_{i=1}^{N}\left(r_{i}-1\right)=2(n+p-1), \quad \text { onde } V \text { é o indice de ramificação de } f \text {. }
$$

Demonstração: Vamos classificar os pontos $P_{i}$ em dois tipos:

(a) pontos $P_{j}^{\prime}$ com ordem de ramificação $r_{j}^{\prime}-1$, tais que $z\left(P_{j}^{\prime}\right) \neq \infty$.

(b) pontos indicados por $P_{k}^{\prime \prime}$ com ordem de ramificação $r_{k}^{\prime \prime}-1$, tais que $z\left(P_{k}^{\prime \prime}\right)=\infty$.

Localmente: em $P_{j}^{\prime}, f$ é dada por $z \longmapsto z^{r^{\prime}}$ e $d f=r_{j}^{\prime} z^{r_{j}^{\prime}-1} d z$; e em $P_{k}^{\prime \prime}, f$ é dada por $z \longmapsto \frac{1}{z_{k}^{r_{k}^{\prime \prime}}}$ e $d f=\frac{-r_{k}^{\prime \prime}}{z_{k}^{r_{k}^{\prime \prime}+1}} d z$. Então, $d f$ em $P_{j}^{\prime}$ e $P_{k}^{\prime}$ tem respectivamente, um zero de ordem $r_{j}^{\prime}-1$ e um polo de ordem $r_{k}^{\prime \prime}+1$.

Pelo corolário 1.2.9, $g r[(d z)]=2 p-2$ Logo,

$$
\mathcal{Z}-\mathcal{P}=\sum_{j=1}^{N_{1}}\left(r_{j}^{\prime}-1\right)-\sum_{k=1}^{N_{2}}\left(r_{k}^{\prime \prime}+1\right)=2 p-2, \quad N_{1}+N_{2}=N
$$

Mas $\operatorname{grau}(f)=n$, que por sua vez é igual ao número de polos de $f$. Portanto:

$$
\sum_{k=1}^{N_{2}} r_{k}^{\prime \prime}=n
$$

Segue-se,

$$
\begin{aligned}
& \sum_{j=1}^{N_{1}}\left(r_{j}^{\prime}-1\right)-\sum_{k=1}^{N_{2}}\left(r_{k}^{\prime \prime}+1\right)=\sum_{j=1}^{N_{1}}\left(r_{j}^{\prime}-1\right)+\sum_{k=1}^{N_{2}}\left(r_{k}^{\prime \prime}-1\right)-2 \sum_{k=1}^{N_{2}} r_{k}^{\prime \prime}=2 p-2, \quad \mathrm{e} \\
& \text { então: } \quad \sum_{j=1}^{N_{1}}\left(r_{j}^{\prime}-1\right)+\sum_{k=1}^{N_{2}}\left(r_{k}^{\prime \prime}-1\right)=2 n+2 p-2 \Rightarrow \sum_{i=1}^{N}\left(r_{i}-1\right)=2(n+p-1)
\end{aligned}
$$

Observação: A fórmula dada em 1.5 é chamada relação de Riemann

Teorema 1.2.14 ("Gap" de Weierstrass) Seja M uma superfície de Riemann compacta de gênero $p \geq 1$. Existem exatamente $p$ números inteiros $n_{i}$ satisfazendo $0<n_{1}<n_{2}<\ldots<n_{p}<2 p$, tais que eles não podem ser especificados como ordens de polos de uma função meromorfa sobre $M$. 
Demonstração: ver [24]

\section{Observações:}

(1) Se $p=0$, a superfície compacta $M$ é homeomorfa a esfera, e como as funções meromorfas sobre $M$ são funções racionais, o teorema não se aplica.

(2) Se $p=1$, temos $0<n_{1}<2 \operatorname{logo} n_{1}=1$, e não existe função meromorfa com um polo simples em $P \in M$.

Seja $D=P^{n}$, entào $\operatorname{gr}\left[P^{n}\right]=n$, se $n>p \Rightarrow n-p \geq 1$. Pelo teorema de Riemann-Roch,

$$
r\left[\frac{1}{P^{n}}\right]=\operatorname{gr}\left[P^{n}\right]+i\left[P^{n}\right]-p+1 \Rightarrow r\left[\frac{1}{P^{n}}\right]=n-p+1+i\left[P^{n}\right] \geq i\left[P^{n}\right]+2
$$

Se $i\left[P^{n}\right] \geq 0$, então $r\left[\frac{1}{P^{n}}\right] \geq 2$. Daí podemos encontrar uma função meromorfa pertencente a $L\left[\frac{1}{P^{n}}\right]$, cuja única singularidade é um polo de ordem no máximo $n$ em $P$. Se $n=p$, existe $f \neq$ cte, $f \in L\left[\frac{1}{P^{p}}\right]$ somente quando $i\left[P^{p}\right]>0$.

Teorema 1.2.15 Existe somente um número finito de pontos $P$ sobre uma superfície de Riemann compacta de gênero $p$, em que $i\left[P^{p}\right]>0$.

Definição 1.2.16 Chama-se ponto de Weierstrass de uma superfície de Riemann compacta de gênero $p$ a um ponto $P$ tal que exista uma função meromorfa não constante cuja única singularidade é um polo em $P$ de ordem menor ou igual ao gênero p. Isto só ocorre nos pontos em que $i\left[P^{p}\right]>0$.

Obviamente, se $p=0$ ou $p=1$, não existem pontos de Weierstrass. Se $p \geq 2$, sempre existem pontos de Weierstrass. Mais precisamente, se $W$ indica o número de pontos de Weierstrass, então $2 p+2 \leq W \leq p^{3}-p$ ver [7].

Definição 1.2.17 Uma superfície de Riemann compacta de gênero $p, M$ é hiperelíptica se existe um divisor $D=P_{1}^{\mu_{1}} P_{2}^{\mu_{2}} \ldots P_{m}^{\mu_{m}}, \mu_{i} \geq 0$ sobre $M$ com :

$$
\operatorname{gr}[D]=2, \quad r\left[\frac{1}{D}\right] \geq 2 \quad \text { e } \quad p \geq 2
$$


Em outras palavras, a definição significa que uma superfície de Riemann compacta $M$ é hiperelíptica se, e somente se, existe uma função meromorfa não constante $f$ de grau 2 sobre $M$.

\subsection{Funções Algébricas.}

O objetivo desta seção é estabelecer uma relação entre superfície de Riemann abstrata como na definiçào 1.1.2 da seção 1.1, e as superfícies de Riemann de equações algébricas.

\subsubsection{Superfícies de Riemann de Funções Algébricas}

Seja $M$ uma superfície de Riemann compacta de gênero $p$ e $z: M \longrightarrow \mathbb{C}$ uma função meromorfa não constante de grau $n$ sobre $M$. De fato, esta função existe já que para todo ponto $P \in M$, se considerarmos o divisor $D=P^{p+1}$, pelo teorema de Riemann-Roch 1.2.7, $r\left[1 / P^{p+1}\right] \geq 2$. Logo existe uma tal função $z$ para algum $n \leq p+1$. Seja $w$ uma outra função meromorfa sobre $M$. Removemos de $\mathbb{C} \cup\{\infty\}$ os pontos: $\infty$, as imagens dos pontos de ramificação de $z$ e os pontos cuja imagem inversa de $z$ é constituído por polos de $w$. Seja $S^{\prime}$ a esfera de Riemann sem esses pontos; para cada $z^{\prime} \in S^{\prime}, z^{-1}\left(z^{\prime}\right)=\left\{P_{1}, P_{2}, \ldots, P_{n}\right\}$ e $w\left(P_{i}\right) \in \mathbb{C}, i=1, \ldots, n$. Sejam ainda,

$$
\sigma_{\nu}(z):=(-1)^{\nu} \sum_{1 \leq n_{1} \leq \ldots \leq n_{\nu} \leq n} w\left(P_{n_{1}}\right) \ldots w\left(P_{n_{\nu}}\right),
$$

a $\nu$-ésima função simétrica relativa ao $w\left(P_{i}\right)$ e $\mathbb{P}(z, x)$ o polinômio de duas veriáveis dado por:

$$
\mathbb{P}(z, x):=x^{n}+r_{1}(z) x^{n-1}+\ldots+r_{n-1}(z) x+r_{n}(z),
$$

onde $r_{\nu}(z)$ são funções racionais. Temos a definição: 
Definição 1.3.1 Uma função $w=w(z)$ é uma função algébrica, se satisfaz a equação algébrica $\mathbb{P}(z, w(z))=0$.

Teorema 1.3.2 Sobre a superficie de Riemann compacta $M$ sejam, $z=z(P)$ uma função meromorfa de grau n e $w=w(P)$ uma outra função meromorfa sobre $M$. Então w satisfaz uma equação algébrica de grau $n$

$$
w^{n}+\sigma_{1}(z) w^{n-1}+\ldots+\sigma_{n-1}(z) w+\sigma_{n}(z)=0
$$

onde $\sigma_{\nu}(z), \nu=1,2, \ldots, n$, são funções racionais de $z$.

Demonstração: ver [24], pag. 286 ou [14] pag. 225

Teorema 1.3.3 Se z é uma função meromorfa de grau n, sobre uma superfície de Riemann compacta $M$, então existe uma segunda função meromorfa $w$ sobre $M$ que satisfaz a equação algébrica 1.7 onde $\operatorname{IP}(z, x)$ de 1.6 é irredutivel.

Demonstração: ver [24] ou [14] pag. 225

Segue dos teoremas (1.3.2) e (1.3.3) que se $M$ é uma superfície de Riemann compacta, $z$ uma função meromorfa não constante em $M$, e se $w$ é uma outra função meromorfa sobre $M$, então existe um polinômio irredutível $\mathbb{P}(z, w)$ tal que $\mathbb{P}(z(P), w(P))=0$, para todo $P$ pertencente a $M$.

Reciprocamente, dado qualquer equação algébrica como em 1.7 podemos construir uma superfície de Riemann compacta $\bar{R}$ associada a ela.

Definição 1.3.4 Um elemento de função $\left(z_{0}, w\right)$ em uma superfície de Riemann $M$ é uma série de potências

$$
\sum_{n=0}^{\infty}\left(z-z_{0}\right)^{n} \text {, onde } z \text { é uma coordenada local de } M, z(P)=z_{0} \text {, }
$$

a qual converge para uma função analítica $w$ em $\left|z-z_{0}\right|<r(r$ pode ser $\infty)$. 
Na vizinhança de $z(P)=z_{0} \in S^{\prime}$, existem $n$ elementos de função $\left(z, w_{1}(z)\right),\left(z, w_{2}(z)\right), \ldots,\left(z, w_{n}(z)\right)$ distintas, que satisfazem uma equação algébrica $\mathbb{P}\left(z, w_{k}(z)\right)=0, k=1, \ldots, n$, e qualquer continuação analítica de tais elementos de função, satisfazem a mesma equação.

Teorema 1.3.5 Seja $\mathbb{P}(z, w)$ um polinômio irredutivel, e suponhamos que numa vizinhança $V$ de um ponto $z_{0}$ esteja definido um elemento de função $w(z)$ tal que $\mathbb{P}(z, w(z))=0$, para todo $z$ pertencente a $V$. Então:

(1) A superfície de Riemann $\bar{R}$ de $w=w(z)$ é compacta;

(2) Se $w_{1}(z)$ é um elemento de função definido numa vizinhança $V_{1}$ de um certo ponto $z_{1}$ pertencente a $S^{\prime}$, tal que $\mathbb{P}\left(z, w_{1}(z)\right)=0$, para todo $z$ pertencente a $V_{1}$, então $w_{1}$ pode ser obtido de $w(z)$ por prolongamento analítico.

Demonstração: ver [20] pag. 62

Dada uma superfície compacta $M$, existem um elemento de função $(z, w)$ e $\mathbb{P}(z, x)$ tal que $\mathbb{P}(z(P), w(P))=0$ para todo $P$ pertencente a $M$. Por outro lado, pelo teorema (1.3.5) a equação $\mathbb{P}(z, w)=0$ determina uma superfície de Riemann compacta $\bar{R}$; de acordo com o seguinte esquema:

$$
M \longrightarrow(z, w) \longrightarrow \mathbb{P}(z, w)=0 \longrightarrow \bar{R}
$$

Teorema 1.3.6 Qualquer superfície de Riemann compacta $M$ pode ser ser realizada como a superfície de Riemann compacta $\bar{R}$ de uma equação algébrica irredutível $\mathbb{P}(z, w)=0$. Mais precisamente, para qualquer função meromorfa $z=z(P)$ sobre $M$, podemos construir uma função meromorfa $w=w(P)$, de tal modo que a aplicação $P \longrightarrow(z, w)$ é uma bijeção conforme de $M$ sobre a superfície $\bar{R}$ associada a equação irredutível satisfeita por $w$.

\section{Demonstração:}

Seja $\bar{R}$ a superfície de Riemann do teorema 1.3 .5 , e seja a aplicação:

$$
\begin{aligned}
\varphi: M & \longrightarrow \bar{R} \\
P & \longmapsto \varphi(P):=(z(P), w(P))
\end{aligned}
$$


onde $(z, w)$ é um elemento de função de $M$ na vizinhança de $P$ tal que $\mathbb{P}(z(P), w(P))=0$. $\varphi$ é um biholomorfismo. De fato, sejam $P_{1}, P_{2} \in M$, e $z\left(P_{1}\right) \neq z\left(P_{2}\right)$, então os elementos de função são distintos, $\left(z\left(P_{1}\right), w\left(P_{1}\right)\right) \neq\left(z\left(P_{2}\right), w\left(P_{2}\right)\right)$; no caso em que $z\left(P_{1}\right)=z\left(P_{2}\right)$ os elementos de função também são distintos. Portanto, $\varphi$ é injetiva. Além disso, uma coordenada local de $M$ se transforma numa coordenada local da superfície $\bar{R}$, logo temos uma aplicação injetiva e conforme. Pelo teorema (1.1.6), temos que esta aplicação também é sobrejetora.

Então todas as superfícies de Riemann compactas definidas abstratamente podem ser realizadas por meio de uma equação algébrica.

\subsubsection{Alguns Exemplos de Superfícies de Funções Algébricas}

As funções algébricas mais simples são aquelas definidas por uma equação da forma $p_{0}(z) w+p_{1}(z)=0, p_{0}(z) \neq 0$, onde $p_{0}(z)$ e $p_{1}(z)$ são polinômios. Então, $w=-p_{1}(z) / p_{0}(z)=r_{1}(z)$ é uma função racional de $z$. Neste caso $w: \mathbb{C} \cup\{\infty\} \longrightarrow \mathbb{C}$ é uma funçào regular exceto para um número finito de polos.

Vamos analisar agora as funções algébricas definidas por equações de grau $2 \mathrm{em}$ $w ; p_{0}(z) w^{2}+p_{1}(z) w+p_{2}(z)=0$. Obteremos, portanto, uma superfície de Riemann construída com 2 folhas (regiões onde cada ramo de $w(z)$ está bem definido). Pondo, $\zeta:=2 p_{0}(z) w(z)+p_{1}(z)$ e $p(z):=p_{1}{ }^{2}(z)-4 p_{0}(z) p_{2}(z)$, obtém-se $\zeta^{2}-p(z)=0$. (1) Se $p(z)=z, \sqrt{z}$ não esta bem definida em $\mathbb{C} \cup\{\infty\}$. É fácil ver que $w$ está bem definida na região como na figura 1.3. Para construir a superfície de Riemann $\bar{R}$ de $w=w(z)$, colamos $I_{+}$com $I I_{-}$e $I_{-}$com $I I_{+}$, isto é, colamos o bordo dos cortes da Folha $I$ e Folha $I I$ em cruz. Portanto, $w: \bar{R} \longrightarrow \mathbb{C}$ está bem definida. Aqui $\bar{R}$ é uma superfície de Riemann de duas folhas, e será representada como na figura 1.3. 

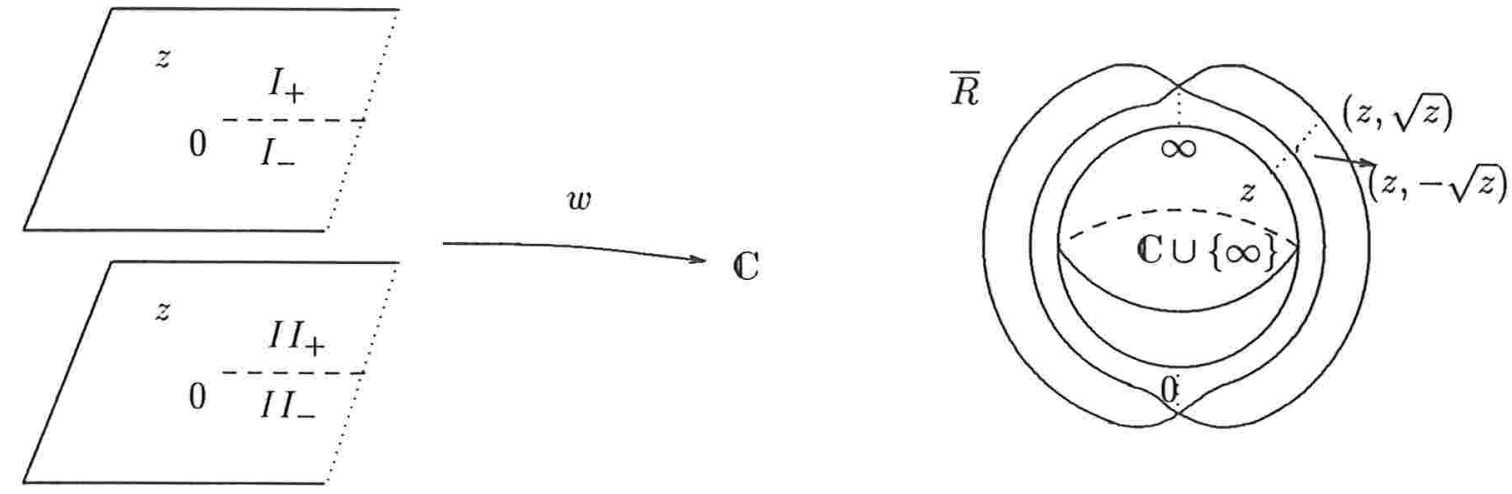

Figura 1.3

Para cada ponto base $z$, existem dois valores para $w=\sqrt{z}$, exceto em $z=0$ e $z=\infty$, que são os pontos de ramificação de $w=\sqrt{z}$.

Cada ponto de $\bar{R}$ é representado por um par $(z, w)$, neste caso $(z, \sqrt{z})$ pertence a folha $I$ e $(z,-\sqrt{z})$ pertence a folha $I I$.

Mostraremos agora que a superfície de Riemann de duas folhas $\bar{R}$ pode ser aplicada homeomorficamente sobre a esfera $S^{2}(1)$, superfície compacta de gênero $p=0$. Para estabelecer este homeomorfismo, consideremos a inversa de projeção estereográfica para as duas folhas de $w=\sqrt{z}$ veja figura 1.4 , depois suponha que $S^{2}(1)$ cortado ao longo do meridiano possa ser deformada em um hemisfério, rotacionando um dos hemisférios, de modo que seja possível colar os bordos com sinais opostos.
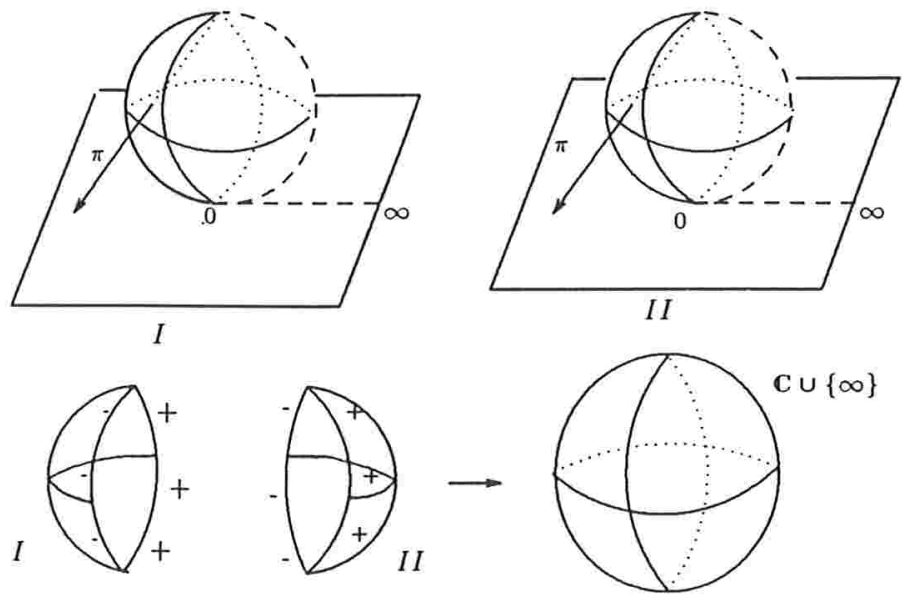


\section{Figura 1.4}

Quando, $p(z)=a_{0} z+a_{1}, a_{0} \neq 0, a_{0}, a_{1} \in \mathbb{C}$, nada muda essencialmente, basta fazer o corte de $z=-\frac{a_{0}}{a_{1}}$ para $z=\infty$ no lugar de $z=0$ para $\infty$.

(2) Se $w^{2}=a_{0} z^{2}+a_{1} z+a_{2}, a_{0} \neq 0$ e $a_{1}^{2}-4 a_{2} a_{0} \neq 0$. Por fatoração, $w^{2}=a_{0}\left(z-r_{1}\right)\left(z-r_{2}\right)$. Aqui também não tem grandes mudanças, salvo que as duas folhas são obtidas fazendo um corte de $r_{1}$ para $r_{2}$ no plano $z$, os quais podem ser levados pela transformação $\tau(z)=\frac{z-r_{1}}{z-r_{2}}$ à $\tau=0$ e $\tau=\infty$. E segue como antes que a superfície de Riemann de $w=\sqrt{a_{0}\left(z-r_{1}\right)\left(z-r_{2}\right)}$ é homeormorfa a $S^{2}$.

(3) A superfície homeomorfa a superfície de Riemann da função algébrica definida por $w^{2}=a_{0}\left(z-r_{1}\right)\left(z-r_{2}\right)\left(z-r_{3}\right)$, onde $r_{1}, r_{2}, r_{3}$ são distintos, é topologicamente diferente dos casos anteriores. Neste caso os pontos de ramificação são $r_{1}, r_{2}, r_{3}$ e $\infty$. $\mathrm{E}$ as duas folhas são obtidas por meio de cortes ligando $r_{1}$ para $r_{2}$ e de $r_{3}$ para $\infty$. Pictoricamente temos:

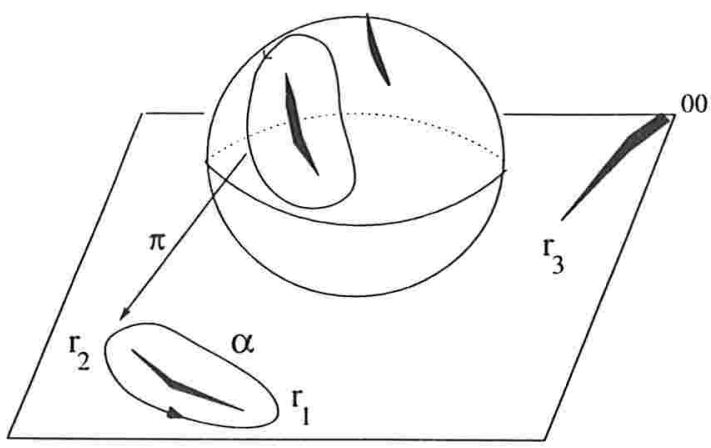

I

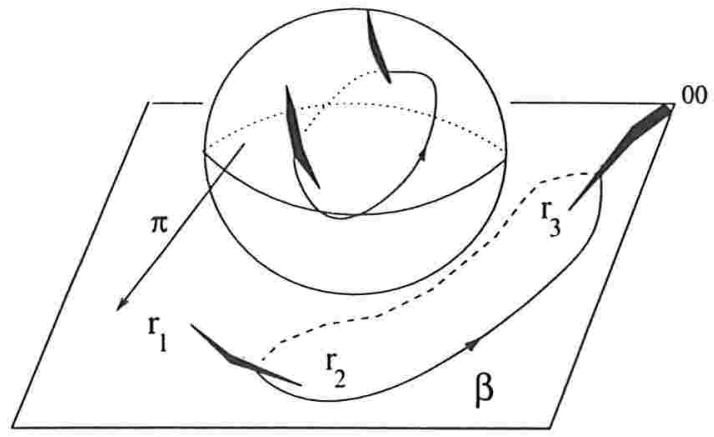

II

Figura 1.5

Seja $\alpha$ a curva fechada dando uma volta em torno do corte feito de $r_{1}$ para $r_{2}$ em uma folha de $\bar{R}$. por exemplo a folha $I$, esta curva existe porque o cruzamento entre as folhas é feito somente a través dos cortes. Seja $\beta$ uma curva fechada iniciando de um ponto sobre um dos lados do corte feito de $r_{1}$ para $r_{2}$ na folha $I I$ e une a um 
ponto sobre um lado do corte de $r_{3}$ para $\infty$, ainda na folha $I I$. E retorna ao ponto inicial pela folha $I$. Estas curvas correspondem a uma base para homologia de $\bar{R}$.

Esticando os cortes até formar tubos e rotacionando uma das esferas a fim de que o bordo + de um tubo é oposto ao bordo - de outro tubo para obter a superfície de Riemann de $w$, a qual é homeomorfa a um toro.
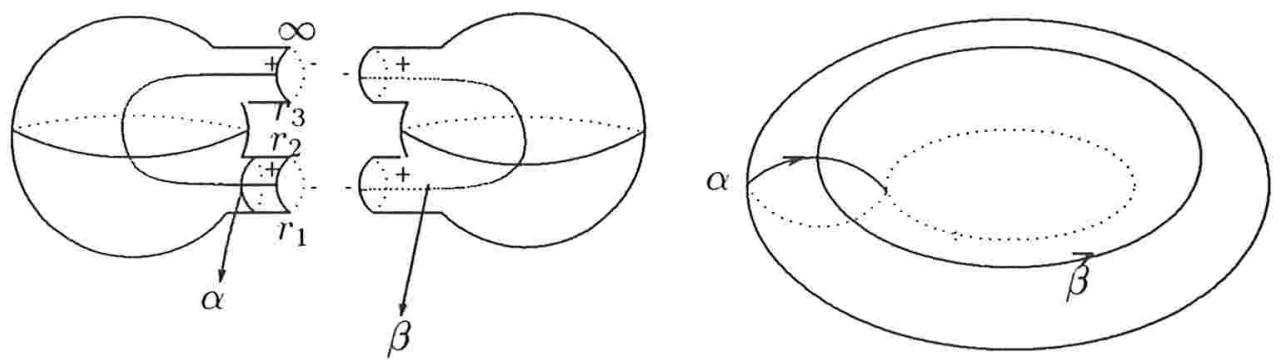

\section{Figura 1.6}

A situação é semelhante quando $w^{2}=w^{2}=a_{0}\left(z-r_{1}\right)\left(z-r_{2}\right)\left(z-r_{3}\right)\left(z-r_{4}\right)$, onde $r_{1}, r_{2}, r_{3}, r_{4}$ são distintos. Neste caso os cortes são feitos entre $r_{1}$ e $r_{2}$ e entre $r_{3}$ e $r_{4}$. Uma representação esquemática da superfície de Riemann acima pode ser dada pela figura $1 . \tau$.

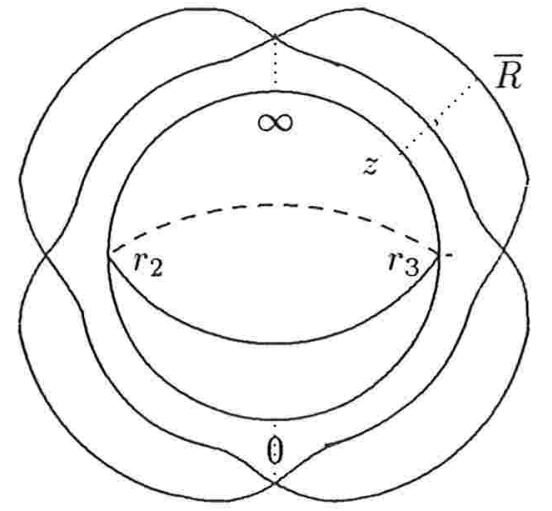

Figura 1.7 
(4) Se $w^{2}=a_{4}\left(z-r_{1}\right)\left(z-r_{2}\right)\left(z-r_{3}\right)\left(z-r_{4}\right)\left(z-r_{5}\right), r_{i} \neq r_{j}$ para $i \neq j, i, j=1, \ldots, 5$. Temos:

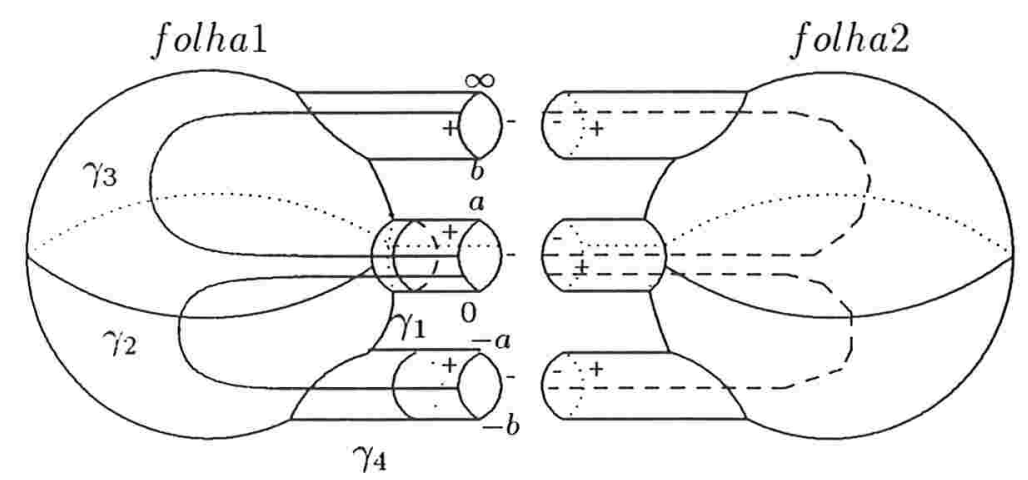

Figura 1.8

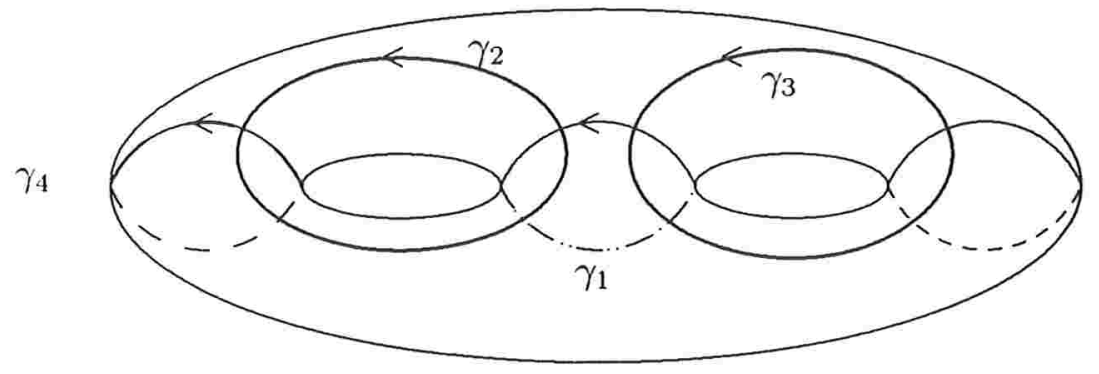

Figura 1.9

(5) Para completar a discussão do caso especial $w^{2}-p(z)=0$, fazemos $w=w(z)$ definida por:

$$
w^{2}=a_{0}\left(z-r_{1}\right)\left(z-r_{2}\right) \ldots\left(z-r_{n}\right) \quad \text { com } r_{i} \neq r_{j}, i \neq j, i, j=1, \ldots, n
$$

Neste caso temos uma superfície de Riemann de duas folhas com pontos de ramificação $r_{1}, \ldots, r_{n}$, e se $n$ é impar, $z=\infty$ é também um ponto de ramificação. Para obter as duas folhas da superfície de Riemann, separamos os pontos em pares $\left(r_{1} r_{2}\right),\left(r_{3} r_{4}\right), \ldots,\left(r_{n-1} r_{n}\right)$ e fazemos os cortes unindo $r_{1}$ a $r_{2}, r_{3}$ a $r_{4}, \ldots$ e $r_{n-1}$ a 
$r_{n}$ se $n$ é par. Se $n$ é impar o último corte é feito unindo $r_{n}$ ao $\infty$.

Observemos que quando $n$ é par, temos $\frac{n}{2}$ tubos e quando $n$ é impar temos $\frac{n+1}{2}$ tubos. Então o gênero $p$ da superfície de Riemann $\bar{R}$ é dado por:

$$
p=\left\{\begin{array} { l l } 
{ \frac { n } { 2 } - 1 , } & { \text { se n é par } } \\
{ \frac { n + 1 } { 2 } - 1 , } & { \text { se n é impar } }
\end{array} \quad \text { isto é, } \left\{\begin{array}{ll}
\frac{n-2}{2}, & n=2 m+2, m \in \mathbb{N} \\
\frac{n-1}{2}, & n=2 m+1
\end{array}\right.\right.
$$

Logo,

$$
\begin{aligned}
& p=\frac{n-2}{2}=\frac{2 m+2-2}{2}=m \\
& p=\frac{n-1}{2}=\frac{2 m+1-1}{2}=m
\end{aligned}
$$

portanto, $p=m$ e

$$
\begin{gathered}
w^{2}=a\left(z-r_{1}\right)\left(z-r_{2}\right) \ldots\left(z-r_{2 p+1}\right) \quad \text { ou } \\
w^{2}=a\left(z-r_{1}\right)\left(z-r_{2}\right) \ldots\left(z-r_{2 p+2}\right),
\end{gathered}
$$

realizam uma superfície de Riemann $\bar{R}$ de gênero $p$, que é topologicamente equivalente a uma esfera com $p$ alças, que por sua vez é homeomorfa a um $p$-toro.

Observação: Quando $w^{2}=Q(z)$, onde $Q(z)=\left(z-e_{1}\right)\left(z-e_{2}\right) \ldots\left(z-e_{k}\right)$, podemos supor grau de $Q(z)$ impar, $k=2 m+1, m \in \mathbb{N}$, pois se $k$ for par, $k=2 m+2$. basta fazer $e_{k} \rightarrow \infty$ por uma mudança de coordenadas do tipo $\zeta=\frac{1}{\left(z-e_{k}\right)}$ e trocar $w$ por

$$
w_{1}=\frac{\zeta^{m+1} w}{\left[\prod_{j=1}^{k-1}\left(e_{j}-e_{k}\right)\right]^{1 / 2}}
$$

Logo, $w_{1}{ }^{2}=\left(\zeta-c_{1}\right)\left(\zeta-c_{2}\right) \ldots\left(\zeta-c_{k-1}\right)$, i.e. $w_{1}^{2}=\left(\zeta-c_{1}\right)\left(\zeta-c_{2}\right) \ldots\left(\zeta-c_{2 m+1}\right)$ onde $c_{1}, c_{2}, \ldots, c_{2 m+1}$ são distintos. Assim podemos considerar

$$
w_{1}^{2}=\left(z-e_{1}\right)\left(z-e_{2}\right) \ldots\left(z-e_{2 m+1}\right) .
$$

Como $p=\frac{k-1}{2}$, no caso $k$ ímpar, $k=2 m+1$ temos

$$
w_{1}^{2}=\left(z-e_{1}\right)\left(z-e_{2}\right) \ldots\left(z-e_{2 p+1}\right)
$$

Agora vamos construir uma base canônica para homologia da superfície de Riemann $\bar{R}$ da função algébrica $w$ definida por

$$
w=\sqrt{\prod_{j=1}^{2 p+1}\left(z-r_{j}\right)} .
$$


Representamos uma das folhas de $\bar{R}$, (por exemplo a folha $I$, como na figura 1.10 abaixo. Sejam as curvas simples fechadas diferenciáveis $\alpha_{k}, k=1, \ldots p$ dando uma volta em torno dos cortes feitos ligando $r_{2 k-1}$ à $r_{2 k}$ em uma folha de $\bar{R}$ orientada como na figura 1.10. Para completar a base para homologia de $\bar{R}$, escolhemos curvas $\beta_{k}, k=1, \ldots p-1$, iniciando em um ponto sobre um lado inferior do corte feito de $r_{2 k-1}$ a $r_{2 k}$ na folha $I I$, unindo a um ponto sobre o corte feito de $r_{2 k+1}$ à $r_{2 k+2}$ da mesma folha e retornando ao ponto inicial pela folha $I$. Quando $k=p$, a idéia é a mesma feita nos cortes ligando $r_{2 p-1}$ à $r_{2 p}$ e $r_{2 p+1}$ à $\infty$.

Observação: Na figura 1.10, a parte pontilhada das curvas $\beta_{k}$ pertencem a folha $I I$.

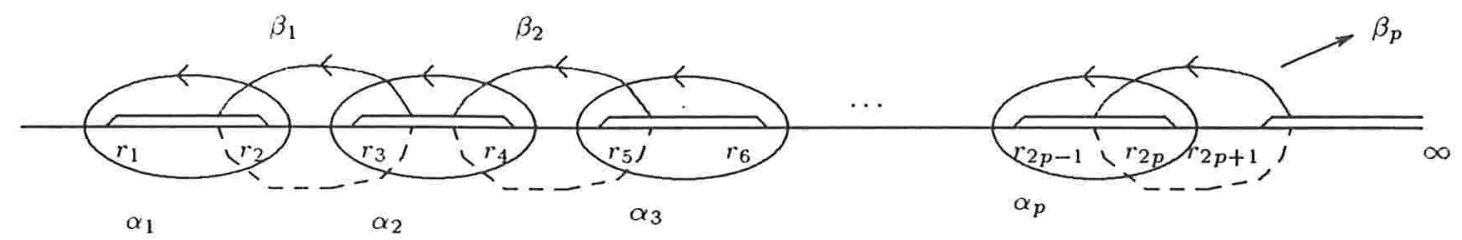

Figura 1.10

O conjunto com $2 p$ curvas dado por:

$$
\left\{\alpha_{1}, \alpha_{2}, \alpha_{3}, \ldots, \alpha_{p}, \beta_{1}, \beta_{2}, \beta_{3}, \ldots, \beta_{p}\right\}
$$

forma uma base para a homologia de $\bar{R}$, isto é, qualquer outra curva fechada simples em $\bar{R}$ pode ser escrita como combinação linear dessas curvas. Outra maneira de ver essas curvas é a seguinte:

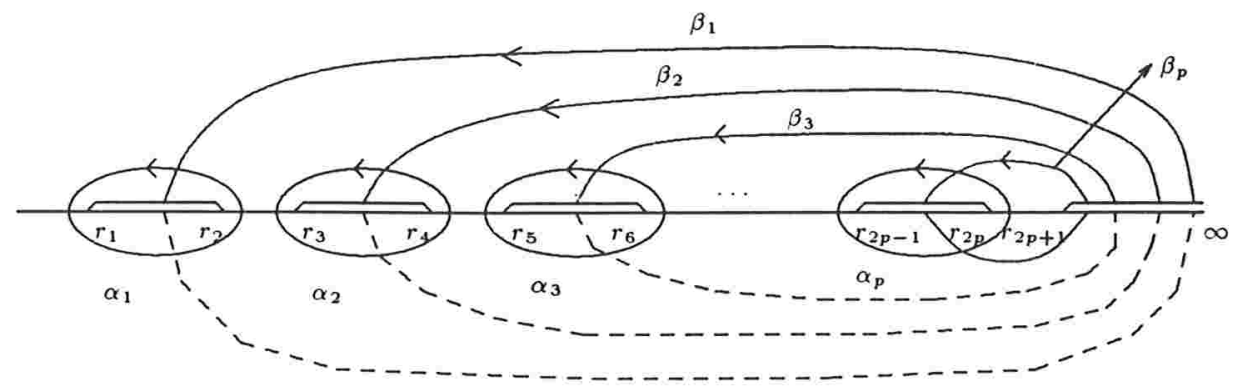

Figura 1.11 


\subsubsection{Superfícies Hiperelípticas e Equações Algébricas}

De acordo com a definição 1.2.17 de superfícies hiperelípticas, existe uma função meromorfa não constante $w$, que satisfaz uma equação algébrica irredutível, $w^{2}=\left(z-e_{1}\right)\left(z-e_{2}\right) \ldots\left(z-e_{k}\right), \quad$ com $e_{1}, e_{2}, \ldots, e_{k} \quad$ distintos $\quad$ e $k=2 p+1$.

Os $k$ pontos $e_{1}, e_{2}, \ldots, e_{k}$, são os pontos de ramificação da superfície $\bar{R}$. Já vimos que se $k$ é ímpar. $z=\infty$ também é um ponto de ramificação. Uma outra maneira de verificar 1.8 é usando a relação de Riemann 1.5 dada no teorema 1.2.13.

$$
V=2(n+p-1), \quad \text { onde neste caso } n=2 .
$$

Se $k$ é par. então $V=k+0, \operatorname{logo}, k=2(2+p-1)$ e, portanto $p=\frac{k-2}{2}$.

Se $k$ é impar, $V=k+1$, portanto $k+1=2+2 p$ e $p=\frac{k-1}{2}$.

Segue que $p \geq 2$ se, e só se, $k>4$. Logo uma superfície hiperelíptica pode ser realizada pela função algébrica

$$
w^{2}=\left(z-e_{1}\right)\left(z-e_{2}\right) \ldots\left(z-e_{k}\right), \quad \text { onde } k>4
$$

Estudaremos agora as diferenciais abelianas. Seja

$$
w^{2}=\left(z-e_{1}\right)\left(z-e_{2}\right) \ldots\left(z-e_{2 p+1}\right)
$$

temos o teorema:

Proposição 1.3.7 As diferenciais

$$
\eta_{1}=\frac{d z}{w}, \quad \eta_{2}=\frac{z d z}{w}, \quad \eta_{3}=\frac{z^{2} d z}{w}, \quad \ldots, \quad \eta_{p}=\frac{z^{p-1} d z}{w}
$$

formam uma base para o espaço das diferenciais de primeira espécie.

Demonstração: ver [24]

Lema 1.3.8 Se $p>0$, não existe um ponto $P_{1} \in M$, no qual todas as diferenciais abelianas de primeira espécie se anulem, isto é, $i\left[P_{1}\right]<p$. 


\section{Demonstração:}

Suponhamos que $i\left[P_{1}\right]=p$, para toda diferencial abeliana $\omega \neq \equiv 0$, onde $\operatorname{ord}_{P_{1}}[(\omega)]>0$. Então, pelo teorema de Riemann-Roch,

$$
r\left[\frac{1}{P_{1}}\right]=1+p-p+1=2 .
$$

Logo, existe uma função meromorfa não constante $f \in L\left[\frac{1}{P_{1}}\right]$, com polo simples em $P_{1}$, isto é, $f$ tem grau 1. Portanto, $f: M \longrightarrow S^{2}(1)$ é uma aplicação conforme injetiva. Então $M$ tem gênero $p=0$, o que contradiz a hipótese.

Proposição 1.3.9 Toda superfície de Riemann compacta $M$ de gênero $p=2$ é hiperelíptica.

\section{Demonstração:}

Pela proposição 1.2 .2 temos, $i[1]=p=2$. Então, $r[1]=0+2-2+1=1$. Seja $\omega \in \Omega\left[P_{1} P_{2}\right]$, logo pela proposição 1.2.6,

$$
i\left[P_{1} P_{2}\right]=r\left[\frac{P_{1} P_{2}}{(\omega)}\right]=r[1]=1 .
$$

Portanto, pelo teorema de Riemann-Roch,

$$
r\left[\frac{1}{P_{1} P_{2}}\right]=2 \text {. }
$$

Agora, para qualquer divisor inteiro $D=P_{1} \ldots P_{n}$, temos que $r\left[\frac{1}{D}\right] \geq 1$. Logo, $i[D] \geq p-n$. Mas, pelo lema 1.3.8, vimos que $i\left[P_{1}\right] \leq p-1$, então, $i\left[P_{1}\right]=p-1=$ $2-1=1$, e portanto.

$$
r\left[\frac{1}{P_{1}}\right]=r\left[\frac{1}{P_{2}}\right]=1 .
$$

Concluímos então que, existe uma função meromorfa $f$ sobre $M$ com polos simples em $P_{1}$ e $P_{2}$, isto é, grau de $f$ é 2 .

\subsection{Funções Elípticas}

Todos os resultados desta seção que não forem demonstrado podem ser encontrados por exemplo em [18]. 


\subsubsection{Definição da Função $\wp$-Weierstrass}

Definição 1.4.1 Uma função $f$ é chamada elíptica com respeito ao reticulado $L\left(w_{1}, w_{2}\right)$ de $\mathbb{C}$, se:

i) $f$ é meromorfa em $\mathbb{C}$;

ii) $f$ é duplamente periódica $\left\{\begin{array}{l}f\left(z+w_{1}\right)=f(z) \\ f\left(z+w_{2}\right)=f(z)\end{array}\right.$

Agora vamos enunciar alguns fatos básicos sobre a teoria das funções elípticas.

Teorema 1.4.2 Não existe função elíptica holomorfa no plano inteiro.

Teorema 1.4.3 A soma dos resíduos de uma função elíptica com respeito a $L\left(w_{1}, w_{2}\right)$ nos polos $a_{1}, \ldots, a_{k}$ pertencentes ao paralelogramo fundamental $F$ é igual a zero. Isto é, $\sum_{j=1}^{k} \operatorname{Res}\left(f ; a_{j}\right)=0$.

Como consequência imediata desse teorema, temos que $f$ não pode ter um polo simples.

Corolário 1.4.4 A soma das ordens dos polos de uma função elíptica em $F$ é no mínimo 2.

Seja $f$ uma função elíptica com polos $a_{1}, \ldots, a_{k}$ de ordem $\mu_{1}, \ldots, \mu_{k}$ e zeros $z_{1}, \ldots, z_{l}$ de ordem $\nu_{1}, \ldots, \nu_{l}$ no paralelogramo fundamental $F$, e $f(z) \neq 0 \quad \forall z \in \partial F$ (bordo de $F$ ). Segue o teorema,

Teorema 1.4.5 Seja $f$ uma função elíptica em $L\left(w_{1}, w_{2}\right)$, então o número de zeros é igual ao número de polos, levando-se em conta suas respectivas ordens. Isto é, $\sum_{i=1}^{k} \mu_{i}-\sum_{i=1}^{l} \nu_{i}=0$.

Corolário 1.4.6 Um função elíptica $f$ assume qualquer valor um número fixo de vezes digamos $m$, em um paralelogramo fundamental. 
Para demonstrar este corolário basta aplicar o teorema (1.4.5) a função $g(z)=f(z)-\alpha$, onde $\alpha \in \mathbb{C}$ é qualquer constante arbitrária.

Definição 1.4.7 O grau de uma função elíptica $f$, grau(f), é igual ao número de polos ou zeros, levando em conta suas ordens.

Teorema 1.4.8 Seja $f$ uma função elíptica relativa a $L\left(w_{1}, w_{2}\right), F$ o paralelogramo fundamental, $a_{1}, \ldots, a_{k} \in F$ polos de $f$ de ordem $\mu_{1}, \ldots, \mu_{k} ; z_{1}, \ldots, z_{l} \in F$, zeros de $f$ com ordens $\nu_{1}, \ldots, \nu_{l}$. Então $\sum_{j=1}^{k} \mu_{j} a_{i}-\sum_{i=1}^{l} \nu_{i} z_{i}=w$, onde $w=m w_{1}+n w_{2} \in L\left(w_{1}, w_{2}\right)$

A função meromorfa mais simples com respeito ao reticulado $L\left(w_{1}, w_{2}\right)$ é uma função com polo simples nos pontos $w=m w_{1}+n w_{2}$ e parte principal $\frac{1}{z-w}$. Ela é chamada função $\zeta$ de Weierstrass.

Proposição 1.4.9 A função $\zeta$ relativa ao reticulado $L\left(w_{1}, w_{2}\right)$ definida por

$$
\zeta(z):=\frac{1}{z}+\sum_{w \neq 0}\left\{\frac{1}{z-w}+\frac{1}{w}+\frac{z}{w^{2}}\right\}, \quad \text { onde } w \in L\left(w_{1}, w_{2}\right)
$$

é meromorfa em $\mathbb{C}$, com polo de ordem 1 em $L\left(w_{1}, w_{2}\right)$ e parte principal $\frac{1}{z-w}$.

Observação: A função $\zeta$ de Weierstrass não é elíptica.

A função elíptica mais simples relativa ao reticulado $L\left(w_{1}, w_{2}\right)$ é uma função com polo de ordem 2 em $w \in L\left(w_{1}, w_{2}\right)$ e regular nos outros pontos. É a função $\wp$-Weierstrass da proposição que segue.

Proposição 1.4.10 A função $\wp$ relativa à $L\left(w_{1}, w_{2}\right)$ definida por

$$
\wp(z):=\frac{1}{z^{2}}+\sum_{w \neq 0}\left\{\frac{1}{(z-w)^{2}}-\frac{1}{w^{2}}\right\}, \quad \text { onde } w \in L\left(w_{1}, w_{2}\right)
$$

é uma função elíptica par com polos de ordem 2 nos pontos do reticulado $w \in L\left(w_{1}, w_{2}\right)$ e holomorfa em $\mathbb{C} \backslash L\left(w_{1}, w_{2}\right)$. Além disso vale $\wp(z)=-\zeta^{\prime}(z)$. 
Da relação, $\zeta^{\prime}(z)=-\wp(z)$, segue-se $\zeta\left(z+w_{j}\right)=\zeta(z)+c$, onde $c$ é uma constante e $j=1,2$.

Por conveniência faremos,

$$
2 \eta_{j}:=\zeta\left(z+w_{j}\right)-\zeta(z), \quad j=1,2
$$

\subsubsection{Relação de Legendre}

Veremos agora como as constantes $\eta_{1}$ e $\eta_{2}$ de 1.9 são relacionadas com $w_{1}, w_{2}$. Consideremos o paralelogramo fundamental $F$ de modo que $z=0$ seja seu centro como na figura 1.12 abaixo. Aplicando o teorema do resíduo, obtemos:

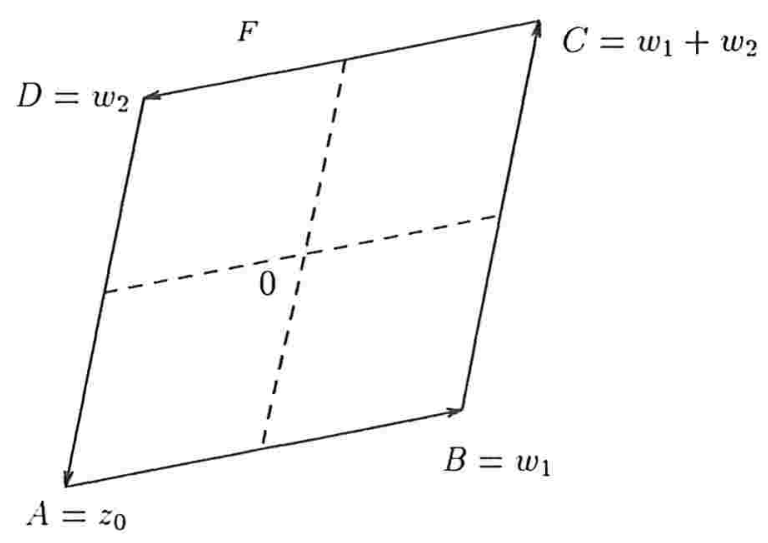

Figura 1.12

$$
\int_{\partial F} \zeta(z) d z=2 \pi i \operatorname{Res}(\zeta(z) ; 0)=2 \pi i
$$

Por outro lado, as relações (1.9) implicam que

$$
\begin{aligned}
& \int_{\partial F} \zeta(z) d z=\left\{\left(\int_{\overline{A B}} \zeta(z) d z+\int_{\overline{C D}} \zeta(z) d z\right)+\left(\int_{\overline{B C}} \zeta(z) d z+\int_{\overline{D A}} \zeta(z) d z\right)\right\} \\
& \int_{\overline{A B}} \zeta(z) d z+\int_{\overline{C D}} \zeta(z) d z=\int_{\overline{A B}} \zeta(z) d z+\int_{\overline{B A}} \zeta\left(z+w_{2}\right) d z
\end{aligned}
$$




$$
\begin{aligned}
& =\int_{\overline{A B}} \zeta(z) d z-\int_{\overline{A B}} \zeta(z) d z-2 \eta_{2} \int_{\overline{A B}} d z=-2 \eta_{2} w_{1} \\
\int_{\overline{B C}} \zeta(z) d z+\int_{\overline{D A}} \zeta(z) d z & =\int_{\overline{A D}} \zeta\left(z+w_{1}\right) d z+\int_{\overline{D A}} \zeta(z) d z \\
& =\int_{\overline{A D}} \zeta(z) d z+2 \eta_{1} \int_{\overline{D A}} d z-\int_{\overline{A D}} \zeta(z) d z=2 \eta_{1} w_{2}
\end{aligned}
$$

Portanto:

$$
\begin{gathered}
\int_{\partial F} \zeta(z) d z=2 \eta_{1} w_{2}-2 \eta_{2} w_{1} \Rightarrow 2\left(\eta_{1} w_{2}-\eta_{2} w_{1}\right)=2 \pi i \\
\eta_{1} w_{2}-\eta_{2} w_{1}=\pi i \quad(\text { relação de Legendre })
\end{gathered}
$$

É fácil ver que $\zeta(-z)=-\zeta(z)$, basta usar na definição de $\zeta(z)$ um rearranjo nos termos da soma. já que esta é absolutamente convergente no disco $|z| \geq \rho$ e $|w|>2 \rho$. Podemos expressar as constante $\eta_{1}$ e $\eta_{2}$ em termos de valores de $\zeta(z)$. De fato, se $z=w_{1} / 2$, então

$$
\begin{aligned}
& \zeta\left(\frac{-w_{1}}{2}\right)=-\zeta\left(\frac{w_{1}}{2}\right) \quad \text { e } \\
& \zeta\left(\frac{w_{1}}{2}\right)=\zeta\left(\frac{-w_{1}}{2}+w_{1}\right)=\zeta\left(\frac{-w_{1}}{2}\right)+2 \eta_{1} \quad \text { por } 1.9 \\
& \Rightarrow \zeta\left(\frac{w_{1}}{2}\right)=-\zeta\left(\frac{w_{1}}{2}\right)+2 \eta_{1} \Rightarrow \eta_{1}=\zeta\left(\frac{w_{1}}{2}\right)
\end{aligned}
$$

Similarmente, $\eta_{2}=\zeta\left(\frac{w_{2}}{2}\right)$.

Reescrevendo a relação de Legendre, temos

$$
\zeta\left(\frac{w_{1}}{2}\right) w_{2}-\zeta\left(\frac{w_{2}}{2}\right) w_{1}=\pi i
$$

Proposição 1.4.11

Os únicos zeros de $\rho^{\prime}(z):=\frac{-2}{z^{3}}+\sum_{w \neq 0}\left\{\frac{-2}{(z-w)^{3}}\right\}$ no paralelogramo fundamental $F$ são os zeros simples $z_{1}=\frac{w_{1}}{2}, \quad z_{2}=\frac{w_{1}+w_{2}}{2}$ e $z_{3}=\frac{w_{2}}{2}$. 


\section{Demonstração:}

É fácil ver que $\wp^{\prime}(z)$ é uma função elíptica impar. Sejam

$$
z_{1}=\frac{w_{1}}{2}, \quad z_{2}=\frac{w_{1}+w_{2}}{2} \text { e } z_{3}=\frac{w_{2}}{2}
$$

pontos de $F$ como na figura 1.13.

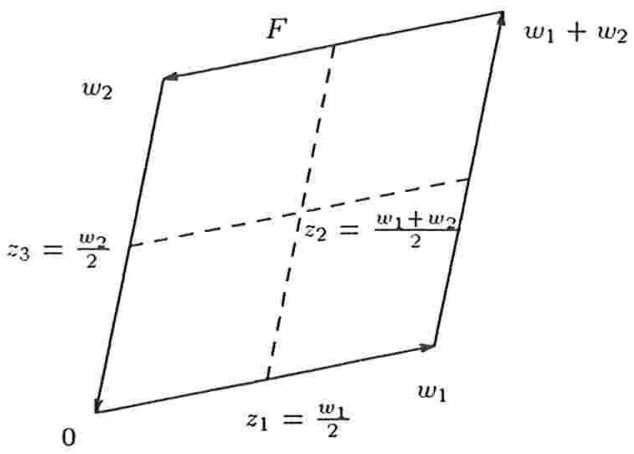

Figura 1.13

Assim:

$$
\left.\begin{array}{l}
\wp^{\prime}\left(-\frac{w_{1}}{2}\right)=-\wp^{\prime}\left(\frac{w_{1}}{2}\right), \\
\wp^{\prime}\left(\frac{w_{1}}{2}\right)=\wp^{\prime}\left(w_{1}-\frac{w_{1}}{2}\right)=\wp^{\prime}\left(-\frac{w_{1}}{2}\right)=-\wp^{\prime}\left(\frac{w_{1}}{2}\right)
\end{array}\right\} \Rightarrow 2 \wp^{\prime}\left(\frac{w_{1}}{2}\right)=0 \Rightarrow \wp^{\prime}\left(\frac{w_{1}}{2}\right)=0 .
$$

Então $z_{1}=\frac{w_{1}^{\prime}}{2}$ é um zero de ordem $\mu_{1}$ de $\wp^{\prime}$. De modo análogo, $z_{2}$ e $z_{3}$ são zeros de $\wp^{\prime}$ de ordem $\mu_{2}$ e $\mu_{3}$ respectivamente. O único polo de $\wp^{\prime}$ em $F$ é assumido em $z=0$ e é de ordem $\nu_{1}=3$. Pelo teorema 1.4.5, $\mu_{1}+\mu_{2}+u_{3}=\nu_{1}$, logo $\mu_{1}+\mu_{2}+\mu_{3}=3$, e portanto, $\mu_{1}=\mu_{2}=\mu_{3}=1$, já que $\mu_{i} \geq 1$. E pelo teorema 1.4.8, se existisse mais um zero, digamos $z_{4}=m_{1} w_{1}+n_{1} w_{2}$, então $\left(m_{1}+1\right) w_{1}+\left(n_{1}+1\right) w_{2}=m w_{1}+n w_{2} \in F$. Logo, $m=n=1$ e $m_{1} w_{1}+n_{1} w_{2}=0, \operatorname{logo} m_{1}=0$ e $n_{1}=0$, e portanto, $z_{4}=0$, contradição pois $a=0$ é um polo de ordem 3 de $\wp^{\prime}$.

Usaremos daqui para a frente a notação:

$$
e_{1}:=\wp\left(\frac{w_{1}}{2}\right), \quad e_{2}:=\wp\left(\frac{w_{1}+w_{2}}{2}\right) \quad \text { e } \quad e_{3}:=\wp\left(\frac{w_{2}}{2}\right)
$$




\subsubsection{A Equação Diferencial da Função $\wp$.}

Da convergência absoluta da série que define $\zeta(z)$ na $|z|<|w|$, onde $|w| \geq$ $2 R, R>0$ e do fato, $\zeta(-z)=-\zeta(z)$, temos,

$$
\zeta(z)=\frac{1}{z}-\left(\sum_{w \neq 0} \frac{1}{w^{4}}\right) z^{3}-\left(\sum_{w \neq 0} \frac{1}{w^{6}}\right) z^{5}-\ldots
$$

Sendo $G_{k}:=\sum_{w \neq 0} \frac{1}{w^{k}}, k=4,6,8, \ldots, \quad$ temos $\zeta(z)=\frac{1}{z}-\sum_{k=2}^{\infty} G_{2 k} z^{2 k-1}$

Pela proposiçào (1.4.10), temos $\wp(z)=-\zeta^{\prime}(z)$. Logo

$$
\begin{aligned}
& \wp(z)=-\left(-\frac{1}{z^{2}}-\sum_{k=2}^{\infty}(2 k-1) G_{2 k} z^{2(k-1)}\right) \\
& \wp(z)=\frac{1}{z^{2}}+\sum_{k=2}^{\infty}(2 k-1) G_{2 k} z^{2(k-1)} \\
& \wp(z)=\frac{1}{z^{2}}+3 G_{4} z^{2}+5 G_{6} z^{4}+\ldots \\
& \wp^{\prime}(z)=-\frac{2}{z^{3}}+6 G_{4} z+20 G_{6} z^{3}+\ldots
\end{aligned}
$$

Por um cálculo simples, tem-se

$$
\wp^{\prime 2}-\left\{4 \wp^{3}-60 G_{4} \wp-140 G_{6}\right\}=o\left(z^{2}\right)
$$

O lado esquerdo da equação (1.14) é elíptica, e a única possibilidade de polo no paralelogramo fundamental $F$ é em $z=0$. Mas o lado direito é regular em $z=0$, e vale zero. Logo pelo teorema (1.4.2),

$$
\wp^{\prime 2}=4 \wp^{3}-60 G_{4 \wp}-140 G_{6}
$$

Pondo $g_{2}:=60 G_{4}=60 \sum_{w \neq 0} \frac{1}{w^{4}}$ e $g_{3}:=140 G_{6}=140 \sum_{w \neq 0} \frac{1}{w^{6}}$, escrevemos

$$
\wp^{\prime 2}=4 \wp^{3}-g_{2} \wp-g_{3}
$$


Vimos na proposição 1.4.11, que $\frac{w_{1}}{2}, \frac{w_{1}+w_{2}}{2}$ e $\frac{w_{2}}{2}$ são zeros simples de $\wp^{\prime}$. logo são raízes do polinômio $4 \rho^{3}-g_{2} \wp-g_{3}$, que por sua vez pode ser fatorado

$$
4 \wp^{3}-g_{2} \wp-g_{3}=4\left(\wp(z)-\wp\left(\frac{w_{1}}{2}\right)\right)\left(\wp(z)-\wp\left(\frac{w_{1}+w_{2}}{2}\right)\right)\left(\wp(z)-\wp\left(\frac{w_{2}}{2}\right)\right)
$$

Portanto,

$$
\wp^{\prime 2}=4\left(\wp-e_{1}\right)\left(\wp-e_{2}\right)\left(\wp-e_{3}\right)
$$

Acabamos de provar a,

Proposição 1.4.12 Seja a função $\wp$ de Weierstrass relativa ao reticulado $L\left(w_{1}, w_{2}\right)$. Temos,

$$
\wp^{\prime 2}=4 \wp^{3}-g_{2} \wp-g_{3} \quad \text { ou } \quad \wp^{\prime 2}=4\left(\wp-e_{1}\right)\left(\wp-e_{2}\right)\left(\wp-e_{3}\right)
$$

Corolário 1.4.13 Sejam $e_{1}, e_{2}, e_{3}$ como acima. Valem as seguintes equações:

(i) $e_{1}+e_{2}+e_{3}=0$.

(ii) $e_{1} e_{2}+e_{2} e_{3}+e_{3} e_{1}=-g_{2} / 4$

(iii) $e_{1} e_{2} \epsilon_{3}=g_{3} / 4$

(iv) $\Delta=16\left(e_{1}-e_{2}\right)^{2}\left(e_{2}-e_{3}\right)^{2}\left(e_{3}-e_{1}\right)^{2}=g_{2}{ }^{3}-27 g_{3}{ }^{2} \neq 0$

Proposição 1.4.14 Sejam as funções elípticas $\wp(z)$ e $\rho^{\prime}(z)$, relativas ao reticulado $L\left(w_{1}, w_{2}\right)$. Vale a fórmula,

$$
\wp\left(z+z_{0}\right)+\wp(z)+\wp\left(z_{0}\right)=\frac{1}{4}\left[\frac{\wp^{\prime}(z)-\wp^{\prime}\left(z_{0}\right)}{\wp(z)-\wp\left(z_{0}\right)}\right]^{2}, \quad z_{0} \neq w \in L\left(w_{1}, w_{2}\right)
$$

chamada fórmula de adição para §.

\subsubsection{A Aplicação Conforme pela Função $\wp$.}

No caso em que $\operatorname{Re}\left\{w_{2} / w_{1}\right\}=0$, o paralelogramo fundamental $F$ é um retângulo ver figura 1.14. Estamos interessados agora, na aplicação conforme da função $\wp$. Sabemos que $\wp$ tem grau 2, i.e. assume qualquer valor duas vezes em $F$, e que $0, \frac{w_{1}}{2}$, $\frac{w_{1}+w_{2}}{2}$ e $\frac{w_{2}}{2}$ são os pontos de ramificação de ordem 1 , já que $z=0$ é um polo de ordem 2 e os outros pontos são zeros simples de $\wp^{\prime}$. 


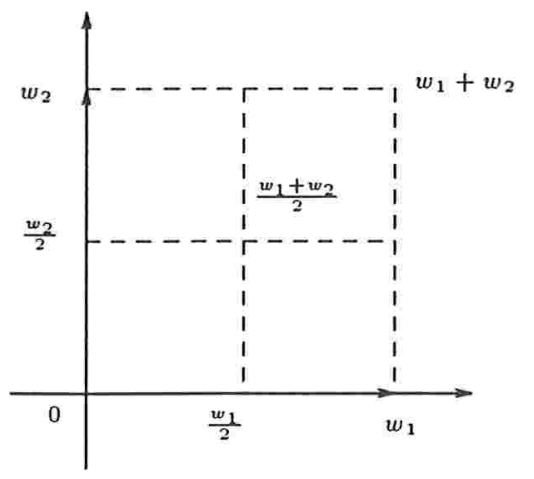

Figura 1.14

Proposição 1.4.15 Seja a função elíptica $\wp \mathrm{em} \mathbb{C}$, relativa ao reticulado $L\left(w_{1}, w_{2}\right)$. Valem as seguintes propriedades:

(i) Se $z=x \in \mathbb{R}$, então $\wp(x) \in \mathbb{R} \cup\{\infty\}$

(ii) Se $z=i y, y \in \mathbb{R}$, então $\wp(i y) \in \mathbb{R} \cup\{\infty\}$

(iii) $\wp\left(x+n w_{2}\right), \wp\left(i y+m w_{1}\right) \in \mathbb{R} \cup\{\infty\}(m, n) \in \mathbb{Z}^{2}$

(iv) $S e z=x+w_{2} / 2, x \in \mathbb{R}$, então $\wp\left(x+w_{2} / 2\right) \in \mathbb{R}$

(v) Se $z=i y+w_{1} / 2, y \in \mathbb{R}$, então $\wp\left(i y+w_{1} / 2\right) \in \mathbb{R}$.

\section{Demonstração:}

Vamos provar primeiro $\overline{\wp(z)}=\wp(\bar{z})$, onde $z=x+i y \in F$. De fato,

$$
\overline{\wp(x+i y)}=\frac{1}{(x-i y)^{2}}+\sum_{w \neq 0}\left\{\frac{1}{(x-i y-\bar{w})^{2}}-\frac{1}{\bar{w}^{2}}\right\}
$$

Por um rearranjo nos termos da série de $\bar{w}=m w_{1}+n \overline{w_{2}}$ para $w=m w_{1}+n w_{2}$, temos

$$
\overline{\wp(x+i y)}=\wp(x-i y)
$$

Em particular se $y=0$, temos $\overline{\wp(x)}=\wp(x) \Rightarrow \wp(x) \in \mathbb{R}$. Como em $z=x=0$, $\wp$ tem um polo, então $\wp(x) \in \mathbb{R} \cup\{\infty\}$.

Suponhamos agora $z=i y$, então pela igualdade $(1.16) \overline{\wp(i y)}=\wp(-i y)$, como $\wp$ é par temos $\wp(-i y)=\wp(i y), \log \overline{\wp(i y)}=\wp(i y)$ e, portanto, $\wp(i y) \in \mathbb{R} \cup\{\infty\} .0$ 
que prova os ítems $(i)$ e $(i i)$. Como $\wp$ é elíptica segue imediatamente o ítem (iii). Seja $z=x+w_{2} / 2, x \in \mathbb{R}$, então

$$
\begin{aligned}
\overline{\wp\left(x+\frac{w_{2}}{2}\right)} & =\frac{1}{\left(\overline{x+\frac{w_{2}}{2}}\right)^{2}}+\sum_{w \neq 0}\left\{\frac{1}{\left(\left(\overline{x+\frac{w_{2}}{2}}\right)-\bar{w}\right)^{2}}-\frac{1}{\bar{w}^{2}}\right\} \\
& =\frac{1}{\left(x-\frac{w_{2}}{2}\right)^{2}}+\sum_{w \neq 0}\left\{\frac{1}{\left(\left(x-\frac{w_{2}}{2}\right)-\bar{w}\right)^{2}}-\frac{1}{\bar{w}^{2}}\right\}
\end{aligned}
$$

por um rearranjo na soma dos termos $\bar{v}$ do reticulado $L\left(w_{1}, w_{2}\right)$ a fim de que a soma fique em $w$, segue

$$
\begin{gathered}
\overline{\wp\left(x+\frac{w_{2}}{2}\right)}=\frac{1}{\left(x-\frac{w_{2}}{2}\right)^{2}}+\sum_{w \neq 0}\left\{\frac{1}{\left(\left(x-\frac{w_{2}}{2}\right)-w\right)^{2}}-\frac{1}{w^{2}}\right\}=\wp\left(x-\frac{w_{2}}{2}\right) \\
\wp\left(x+\frac{w_{2}}{2}\right)=\wp\left(x-\frac{w_{2}}{2}\right)=\wp\left(x+\frac{w_{2}}{2}-w_{2}\right)=\wp\left(x+\frac{w_{2}}{2}\right)
\end{gathered}
$$

Portanto, $\wp\left(x+\frac{w_{2}}{2}\right) \in \mathbb{R}$.

De modo similar temos $\wp\left(i y+\frac{w_{1}}{2}\right) \in \mathbb{R}$.

Corolário 1.4.16 Os pontos de ramificação $e_{1}, e_{2}$ e e $e_{3}$ de $\wp$ são reais e $e_{1}>e_{2}>e_{3}$.

A função $\wp$ aplica o retângulo de vértices $0, \frac{w_{1}}{2}, \frac{w_{1}+w_{2}}{2}, \frac{w_{2}}{2}$ conformemente sobre o lado inferior do plano, região $\wp(I)$ como na figura 1.16. Assim como $\wp$ aplica o retângulo $I I$ sobre o lado superior do plano, região indicada na figura por $\wp(I I)$

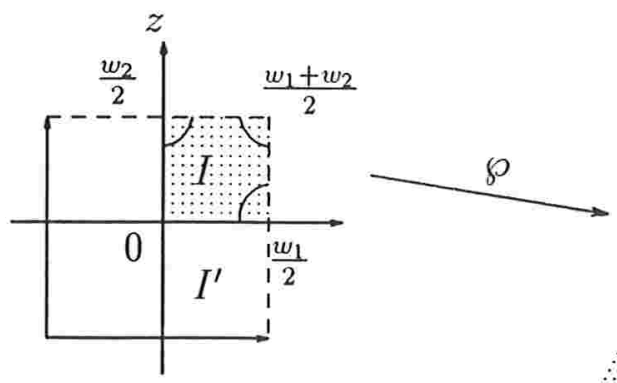

$$
\wp(z)
$$

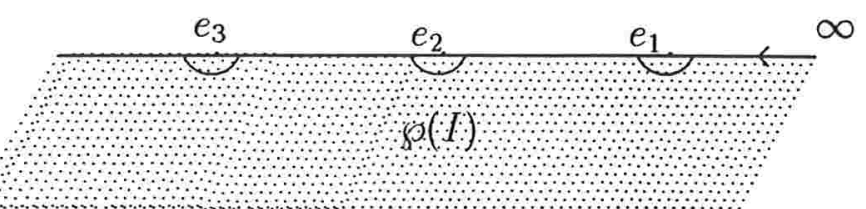


Figura 1.15

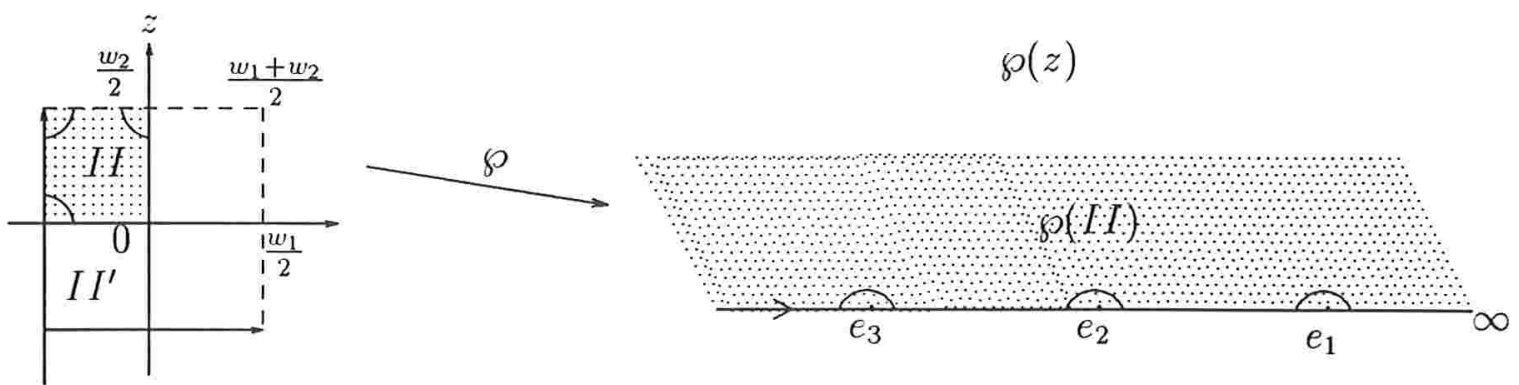

Figura 1.16

A regiào $\wp(I I)$ está ligada a $\wp(I)$ ao longo do arco de $e_{3}$ para $\infty$. Isto nos dá a primeira folha.

Do mesmo modo a região $I^{\prime}$ e $I I^{\prime}$ do paralelogramo fundamental $F$ é aplicada na segunda folha de uma superfície de Riemann. Assim $F$ é aplicado sobre uma superfície de Riemann de duas folhas unidas em cruz ao longo de $e_{1}$ para $\infty$. Ambas folhas são cortadas ao longo de $\left(e_{1} e_{2} e_{3}\right)$.

Qualquer paralelogramo fundamental $F$ é aplicado sobre uma superfície de Riemann de duas folhas cortadas ao longo do arco $\left(e_{1} e_{2} e_{3}\right)$. Essas superfícies são unidas uma a outra ao longo dos cortes $\left(e_{1} e_{2}\right)$ e $\left(e_{2} e_{3}\right)$. Portanto temos,

Proposição 1.4.17 O plano $\mathbb{C}$ pode ser aplicado sobre uma superfície de Riemann de infinitas folhas e com infinitos pontos de ramificação de primeira ordem sobre os pontos $\infty, e_{1}, e_{2}, e_{3}$. 


\subsection{Superfícies Mínimas em $\mathbb{R}^{3}$.}

Nesta seção exporemos alguns resultados clássicos da teoria das superfícies mínimas. $\mathrm{Na}$ primeira subseção faremos uma abordagem local de propriedades de imersão conforme, e na segunda tratamos de conceitos globais de superfícies mínimas..

\subsubsection{Propriedades Locais}

Se $\langle$,$\rangle é a métrica Riemanniana usual do \mathbb{R}^{3}, X: M \longrightarrow \mathbb{R}^{3}$ é uma imersão de uma variedade diferenciável de dimensão 2 em $\mathbb{R}^{3}$, isto é, a diferencial $d X_{P}: T_{P} M \longrightarrow T_{X(P)}\left(\mathbb{R}^{3}\right) \simeq \mathbb{R}^{3}$ é injetiva para todo $P \in M$, onde $T_{P} M$ é o espaço tangente a $M$ no ponto $P$, então $X$ induz uma métrica Riemanniana em $M$, $d s^{2}=X^{*}\langle,\rangle_{P}(\tilde{u} . \tilde{v}):=\left\langle d X_{P}(\tilde{u}), d X_{P}(\tilde{v})\right\rangle_{X(P)}$, onde $\tilde{u}, \tilde{v} \in T_{P} M$.

Definição 1.5.1 Seja $\mathcal{F}_{0} \subset \mathcal{F}$ um atlas de $M$ tal que $\left(U_{\alpha}, V_{\alpha}, z_{\alpha}\right) \in \mathcal{F}_{0}, \quad z_{\alpha}$ são coordenadas isotérmicas de $M$ se para todo $P \in M, \tilde{z}_{\alpha}(P)=z=u+i v \in V_{\alpha}$ vale,

$$
\begin{gathered}
\left\langle\frac{\partial}{\partial u}\left(X \circ z_{\alpha}^{-1}\right)(z), \frac{\partial}{\partial u}\left(X \circ z_{\alpha}^{-1}\right)(z)\right\rangle^{\frac{1}{2}}=\left\langle\frac{\partial}{\partial v}\left(X \circ z_{\alpha}^{-1}\right)(z), \frac{\partial}{\partial v}\left(X \circ z_{\alpha}^{-1}\right)(z)\right\rangle^{\frac{1}{2}}=: \lambda>0 \\
e \quad\left\langle\frac{\partial}{\partial u}\left(X \circ z_{\alpha}^{-1}\right)(z), \frac{\partial}{\partial v}\left(X \circ z_{\alpha}^{-1}\right)(z)\right\rangle=0,
\end{gathered}
$$

onde

$$
X_{u}(z):=\frac{\partial}{\partial u}\left(X \circ z_{\alpha}^{-1}\right)(z), \quad X_{v}(z):=\frac{\partial}{\partial v}\left(X \circ z_{\alpha}^{-1}\right)(z) .
$$

Isto é, $\mathcal{F}_{0}$ é um atlas constituído por coordenadas isotérmicas.

A existência de $\mathcal{F}_{0}$ é garantida pelo teorema:

Teorema 1.5.2 (Existência de Coordenadas Isotérmicas) Seja $M$ uma variedade diferenciável de dimensão 2 com a métrica Riemanniana $\langle$,$\rangle , e z: U \longrightarrow V$ uma coordenada local, onde $U$ é um aberto de $M$, com $P \in U$ e $V$ um aberto de C. Então exitem abertos $V^{\prime}$ e $W$ de $\mathbb{C}$, onde $z(P) \in V^{\prime} \subset V$ e $\varphi: V^{\prime} \longrightarrow W$ um difeomorfismo conforme, tal que $\tilde{z}=\varphi$ o $z$ são coordenadas isotérmicas de $M$. 
Demonstração: ver [23], pag. 458. No caso particular deste teorema, onde $M$ é mínima, podemos encontrar uma demonstração na referência [19] pag. 31.

Observação:

(1) Se $M$ é conexa e orientada e $\mathcal{F}_{0}$ é um atlas de $M$ constituído por coordenadas isotérmicas, então $\mathcal{F}_{0}$ é um atlas holomorfo, e portanto, a estrutura holomorfa que contém $\mathcal{F}_{0}$, torna $M$ uma superfície de Riemann.

(2) Se $M$ é uma variedade diferenciável de dimensão 2 , conexa, orientada e $X: M \longrightarrow \mathbb{R}^{3}$ é uma imersão, então $X$ induz uma estrutura holomorfa sobre $M$, que torna $X$ uma imersão conforme.

Definição 1.5.3 Sejam $M$ uma superfície de Riemann e $X: M \longrightarrow X(M) \subset \mathbb{R}^{3}$ uma imersão conforme, então dizemos que $S=X(M)$ é uma superfície em $\mathbb{R}^{3}$

\section{Observação:}

(1) Desta definição segue que $S=X(M)$ é orientada;

(2) A definição acima não faz restrição a resultados locais ou para superfícies simplesmente conexas;

(3) Como $X^{\prime}: M \longrightarrow X(M)$ é uma imersão, então $S=X(M)$ tem um plano tangente bem definido em cada ponto.

Se $\left(U_{\alpha}, V_{\alpha}, z_{\alpha}\right)$ é uma coordenada local de $M$, tal que $z_{\alpha}(P)=z=u+i v \in V_{\alpha} \subset \mathbb{C}$, e $\left(x_{1}, x_{2}, x_{3}\right)$ são coordenadas em $\mathbb{R}^{3}$, então $X: M \longrightarrow X(M)$ é dada localmente na forma:

$$
X(z)=\left(\left(x_{1} \circ z_{\alpha}^{-1}\right)(z),\left(x_{2} \circ z_{\alpha}^{-1}\right)(z),\left(x_{3} \circ z_{\alpha}^{-1}\right)(z)\right)
$$

A conformidade da aplicação $X$ dada na definição 1.5.3 é expressa em termos da coordenada local $\left(U_{\alpha}, V_{\alpha}, z_{\alpha}\right)$ por:

$$
\left\langle X_{u}, X_{u}\right\rangle^{\frac{1}{2}}=\left\langle X_{v}, X_{v}\right\rangle^{\frac{1}{2}}=\lambda(u, v)>0 \quad \text { e } \quad\left\langle X_{u}, X_{v}\right\rangle=0
$$

e então $z=u+i v$ são coordenadas isotérmicas para $M$.

\section{Observação:}

Sendo $\left(U_{\alpha}, V_{\alpha}, z_{\alpha}\right), g_{11}:=\left\langle X_{u}, X_{u}\right\rangle, g_{22}:=\left\langle X_{v}, X_{v}\right\rangle$ e $g_{12}:=\left\langle X_{u}, X_{v}\right\rangle$ os coeficientes 
da primeira forma fundamental e $b_{11}:=\left\langle X_{u u}, N\right\rangle, b_{22}:=\left\langle X_{v v}, N\right\rangle$ e $b_{12}:=\left\langle X_{u v}, N\right\rangle$ os coeficientes da segunda forma fundamental, então:

$$
h(z):=\frac{g_{22} b_{11}+g_{11} b_{22}-2 g_{12} b_{12}}{2\left(g_{11} g_{22}-g_{12}^{2}\right)}(z)
$$

é a curvatura média de $S$ em $P \in U_{\alpha}$ e $H(z)$ definido por

$$
H(z):=h(z) \cdot N(z) \quad \text { é o vetor curvatura média, }
$$

onde

$$
N(z):=\frac{X_{u} \times X_{v}}{\left\|X_{u} \times X_{v}\right\|}(z)
$$

é o vetor normal de $S$.

Definição 1.5.4 Seja $S=X(M)$ uma superfície do $\mathbb{R}^{3}$. Se o vetor $H$ é identicamente nulo $(H \equiv 0)$, diz-se que $S$ é uma superfície mínima, ou que X é uma imersão minima.

Exemplos de superfícies mínimas:

(a) Superfície de Enneper:

$$
X(u, v)=\left(u-\frac{u^{3}}{3}+u v^{2} ; v-\frac{v^{3}}{3}+v u^{2} ; u^{2}-v^{2}\right), \quad \text { onde } \quad(u, v) \in \mathbb{C}
$$

(b) Catenóide:

$$
X(u, v)=(\cosh (u) \cos (v), \cosh (u) \operatorname{sen}(v), u), \quad \text { onde } \quad(u, v) \in \mathbb{C} \backslash\{0\}
$$

Observação: A razão do adjetivo mínima na definição de superfície mínima é que localmente ela minimiza área dentre uma família de superfícies de classe $C^{2}$ do $\mathbb{R}^{3}$ que tem como bordo uma curva regular fechada.

Em coordenadas isotérmicas muitos dos objetos geométricos de $S=X(M)$ têm expressões analíticas simplificadas. Por exemplo sobre $M$, a métrica induzida de $\mathbb{R}^{3}$ por $X$ é dada em termos da coordenada local $\left(U_{\alpha}, V_{\alpha}, z_{\alpha}\right)$ tal que $z_{\alpha}(P)=z=$ $u+i v \in V_{\alpha}$ por:

$$
d s^{2}=X^{*}(\langle,\rangle)=\lambda^{2}\left(d u^{2}+d v^{2}\right)=\lambda^{2}\left|d z_{\alpha}\right|^{2}
$$


Também temos,

$$
\operatorname{det}\left(g_{i j}\right)=\lambda^{4}>0 \text { e } h=\frac{b_{11}+b_{22}}{2 \lambda^{2}} .
$$

Além disso, se

$$
\Delta:=\frac{\partial^{2}}{\partial u^{2}}+\frac{\partial^{2}}{\partial v^{2}}, \quad \Delta\left(X \circ z_{\alpha}^{-1}\right):=\left(\Delta\left(x_{1} \circ z_{\alpha}^{-1}\right), \Delta\left(x_{2} \circ z_{\alpha}^{-1}\right), \Delta\left(x_{3} \circ z_{\alpha}^{-1}\right)\right),
$$

onde $x_{j} \circ z_{\alpha}^{-1}: V_{\alpha} \longrightarrow \mathbb{R}, j=1,2,3$ são as funções coordenadas de $X$, temos a:

Proposição 1.5.5 Seja $X: M \longrightarrow X(M)$ uma imersão conforme de uma superfície de Riemann $M$ em $\mathbb{R}^{3}$, então

$$
\Delta\left(X \circ z_{\alpha}^{-1}\right)=2 \lambda^{2} H
$$

Demonstração: ver [19].

Observemos que desta proposição e a definição 1.5.4, concluímos que uma superfície é mínima se, e só se as funções coordenadas de $X$ são harmônicas em $V_{\alpha}$.

Corolário 1.5.6 $S=X(M)$ é uma superfície mínima se, e somente se as funções coordenadas de $X(z)$ são harmônicas. Em particular $S$ não é compacta.

No que segue $M$ é uma superfície de Riemann não compacta, $\mathcal{H}_{0}=\left\{\left(U_{\alpha}, v_{\alpha}, z_{\alpha}\right) ; \alpha \in I\right\}$ o Atlas holomorfo que induz a estrutura holomorfa de $M$ e $X: M \longrightarrow X(M)$ uma imersão conforme que define a superfície $S=X(M)$ em $\mathbb{R}^{3}$. Seja $\left(U_{\alpha}, V_{\alpha}, z_{\alpha}\right)$ uma coordenada local de $M$, tal que $z_{\alpha}(P)=z=u+i v$, para todo $P \in U_{\alpha}$. Se

$$
\frac{\partial}{\partial z}:=\frac{1}{2}\left(\frac{\partial}{\partial u}-i \frac{\partial}{\partial v}\right) \quad \text { e } \frac{\partial}{\partial \bar{z}}:=\frac{1}{2}\left(\frac{\partial}{\partial u}+i \frac{\partial}{\partial v}\right)
$$

Então

$$
\Delta=4 \frac{\partial^{2}}{\partial \bar{z} \partial z}=4 \frac{\partial^{2}}{\partial z \partial \bar{z}}
$$

e,

$$
\phi_{j}^{\alpha}(z):=2\left(\frac{\partial}{\partial z}\left(x_{j} \circ z_{\alpha}^{-1}\right)(z)\right) d z_{\alpha}, \quad j=1,2,3
$$


são 1-formas diferenciais para cada coordenada local $z_{\alpha}$ de $M$.

Agora se $S=X(M)$ é uma superfície mínima, segue-se de $1.21,1.23$ e proposição 1.5 .5 que

$$
\frac{\partial}{\partial \bar{z}} \phi_{j}^{\alpha}(z)=\frac{\partial}{\partial \bar{z}}\left[2\left(\frac{\partial}{\partial z}\left(x_{j} \circ z_{\alpha}^{-1}\right)(z)\right) d z_{\alpha}\right]=2\left(\frac{\partial^{2}}{\partial \bar{z} \partial z}\left(x_{j} \circ z_{\alpha}^{-1}\right)(z)\right) d z_{\alpha}=0,
$$

$\operatorname{logo} \phi_{j}^{\alpha}(z)$ é holomorfa em $V_{\alpha}$. Além disso, se $\left(U_{\alpha}, V_{\alpha}, \tilde{z}_{\alpha}\right)$ e $\left(U_{\beta}, V_{\beta}, z_{\beta}\right)$ são duas coordenadas locais de $M$, tais que $z_{\alpha}(P)=z=u+i v$ e $z_{\beta}(P)=\tilde{z}=\tilde{u}+i \tilde{v}$ para todo $P \in U_{\alpha} \cap U_{3}$ vale:

$$
\frac{\partial}{\partial z}(\tilde{z})=\left(z_{\beta} \circ z_{\alpha}^{-1}\right)^{\prime}(z) \frac{\partial}{\partial z}(z)
$$

Então pela definição 1.2 .1 de 1 -formas diferenciais, temos que 1.23 definem 1 -formas diferenciais holomorfas $\phi_{j}(z), j=1,2,3$, globalmente em $M$.

No que segue,

$$
\frac{\partial}{\partial z} X(z):=\frac{\partial}{\partial z}\left(X \circ z_{\alpha}^{-1}\right)(z)=\frac{1}{2}\left(X_{u}-i X_{v}\right)(z)
$$

Então podemos escrever,

$$
\begin{gathered}
\frac{\partial}{\partial z} X(z) d z_{\alpha}=\frac{1}{2}\left(\frac{\partial}{\partial u}\left(x_{1} \circ z_{\alpha}^{-1}\right)-i \frac{\partial}{\partial v}\left(x_{1} \circ z_{\alpha}^{-1}\right), \frac{\partial}{\partial u}\left(x_{2} \circ z_{\alpha}^{-1}\right)-i \frac{\partial}{\partial v}\left(x_{2} \circ z_{\alpha}^{-1}\right)\right. \\
\left.\frac{\partial}{\partial u}\left(x_{3} \circ z_{\alpha}^{-1}\right)-i \frac{\partial}{\partial v}\left(x_{3} \circ z_{\alpha}^{-1}\right)\right)(z) d z_{\alpha} \\
\text { e } \quad \Phi^{\alpha}:=\left(\phi_{1}^{\alpha}, \phi_{2}^{\alpha}, \phi_{3}^{\alpha}\right)=2 \frac{\partial}{\partial z} X(z) d z_{\alpha}
\end{gathered}
$$

Observação: No teorema abaixo, $\langle,\rangle^{\mathbb{C}}$ denota à extensão a $\mathbb{C}^{3}$ do produto interno usual do $\mathbb{R}^{3}$. Sempre que não houver dúvidas, $\langle,\rangle^{\mathbb{C}}$ será ainda indicado por $\langle$,$\rangle .$

Teorema 1.5.7 Sejam $S=X(M)$ uma superfície do $\mathbb{R}^{3}$ e $\left(U_{\alpha}, V_{\alpha}, z_{\alpha}\right)$ uma coordenada local de $M$, onde para todo $P \in U_{\alpha}, z_{\alpha}(P)=z=u+i v \in V_{\alpha}$. Se $\phi_{j}^{\alpha}(z), j=1,2,3$ é dado por 1.23 , então:

(i) $\phi_{j}^{\alpha}$ é holomorfa $\Leftrightarrow x_{j} \circ z_{\alpha}^{-1}$ é harmônica em $V_{\alpha}$, isto é, $\Delta\left(x_{j} \circ z_{\alpha}^{-1}\right)(z)=0, \forall z \in V_{\alpha}$.

(ii) $\left\langle\Phi^{\alpha}, \Phi^{\alpha}\right\rangle^{\mathbb{C}}=\left(\phi_{1}^{\alpha}\right)^{2}+\left(\phi_{2}^{\alpha}\right)^{2}+\left(\phi_{3}^{\alpha}\right)^{2}=0$.

(iii) $S$ é regular para todo ponto $P \in U_{\alpha} \Leftrightarrow\left\langle\Phi^{\alpha}, \overline{\Phi^{\alpha}}\right\rangle^{\mathbb{C}}=\left|\phi_{1}^{\alpha}\right|^{2}+\left|\phi_{2}^{\alpha}\right|^{2}+\left|\phi_{3}^{\alpha}\right|^{2} \neq 0$. 
Reciprocamente, se $V_{\alpha}$ é um aberto de $\mathbb{C}$ simplesmente conexo e $\phi_{j}^{\alpha}, j=1,2,3$ são funções holomorfas em $V_{\alpha}$ satisfazendo (ii) e (iii), então $S=X\left(V_{\alpha}\right)$ dada por:

$$
X(z)=\operatorname{Re}\left\{\int_{z_{0}}^{z} \Phi^{\alpha}\right\}, \quad z_{0} \quad \text { fixo } \quad \text { e } z_{0}, z \in V_{\alpha}
$$

é uma superfície mínima do $\mathbb{R}^{3}$

Demonstração: ver [19] ou [10]

Usando o fato, $z_{\alpha}$ coordenada isotérmica o que implica pelo ítem (ii) do teorema 1.5.7 que $\left(\phi_{1}^{\alpha}\right)^{2}+\left(\phi_{2}^{\alpha}\right)^{2}+\left(\phi_{3}^{\alpha}\right)^{2}=0$, e que $\phi_{1}^{\alpha} \neq i \phi_{2}^{\alpha}$, temos o teorema:

Teorema 1.5.8 Sejam $V_{\alpha}$ um aberto de $\mathbb{C}, g: V_{\alpha} \longrightarrow \mathbb{C}$ uma função meromorfa e $\eta:=f d z_{\alpha}$ uma diferencial holomorfa( i.e, $f: V_{\alpha} \longrightarrow \mathbb{C}$ é uma função holomorfa) tendo a propriedade que em cada ponto onde $g$ tem um polo de ordem $m, \eta$ tem um zero de ordem $2 \mathrm{~m}$. As diferenciais,

$$
\phi_{1}^{\alpha}:=\frac{1}{2}\left(1-g^{2}\right) \eta, \quad \phi_{2}^{\alpha}:=\frac{i}{2}\left(1+g^{2}\right) \eta, \quad \phi_{3}^{\alpha}:=g \eta
$$

são holomorfas em $V_{\alpha}$ e satisfazem $\left(\phi_{1}^{\alpha}\right)^{2}+\left(\phi_{2}^{\alpha}\right)^{2}+\left(\phi_{3}^{\alpha}\right)^{2}=0$. Reciprocamente, quaisquer três funções holomorfas em $V_{\alpha} ; \phi_{1}^{\alpha}, \phi_{2}^{\alpha}$ e $\phi_{3}^{\alpha}$ satisfazendo $\left(\phi_{1}^{\alpha}\right)^{2}+\left(\phi_{2}^{\alpha}\right)^{2}+\left(\phi_{3}^{\alpha}\right)^{2}=0$ podem ser escritas como em 1.26 se $\phi_{1}^{\alpha}-i \phi_{2}^{\alpha} \neq 0$.

A demonstração é imediata se escrevermos

$$
\left(\phi_{1}^{\alpha}+i \phi_{2}^{\alpha}\right)\left(\phi_{1}^{\alpha}-i \phi_{2}^{\alpha}\right)=-\left(\phi_{3}^{\alpha}\right)^{2}
$$

E fizermos

$$
g:=\frac{\phi_{3}^{\alpha}}{\phi_{1}^{\alpha}-i \phi_{2}^{\alpha}} \quad \text { e } \quad \eta:=\phi_{1}^{\alpha}-i \phi_{2}^{\alpha}
$$

Observação: Quando $\phi_{1}^{\alpha}-i \phi_{2}^{\alpha}=0$, de $1.27, \phi_{3}^{\alpha}=0$, logo a função coordenada $x_{3}$ é uma constante e portanto se $x_{3}$ for uma coordenada de uma imersão $X$ que define $S$, esta estaria contida num plano do $\mathbb{R}^{3}$.

Segue de 1.25 e 1.26 que, no caso em que $V_{\alpha}$ é simplesmente conexo e quando $\left|\phi_{1}^{\alpha}\right|^{2}+\left|\phi_{2}^{\alpha}\right|^{2}+\left|\phi_{3}^{\alpha}\right|^{2} \neq 0$, então:

$$
X(z)=\left(\operatorname{Re}\left\{\int_{z_{0}}^{z} \frac{1}{2}\left(1-g^{2}\right) \eta\right\}, \operatorname{Re}\left\{\int_{z_{0}}^{z} \frac{i}{2}\left(1+g^{2}\right) \eta\right\}, \operatorname{Re}\left\{\int_{z_{0}}^{z} g \eta\right\}\right)
$$


é uma imersão conforme mínima. Chamaremos o par $(g, \eta)$, de acordo com 1.26 e 1.29 , de representação de Weierstrass de $S=X\left(V_{\alpha}\right)$

De 1.26 é fácil ver que $\left(\phi_{1}^{\alpha}\right)^{2}+\left(\phi_{2}^{\alpha}\right)^{2}+\left(\phi_{3}^{\alpha}\right)^{2}=0$. Além disso,

$$
\left\langle\Phi^{\alpha}, \overline{\Phi^{\alpha}}\right\rangle=\left|\phi_{1}^{\alpha}\right|^{2}+\left|\phi_{2}^{\alpha}\right|^{2}+\left|\phi_{3}^{\alpha}\right|^{2}=2 g_{11}\left|d z_{\alpha}\right|^{2}=2 \lambda^{2}\left|d z_{\alpha}\right|^{2}
$$

Por outro lado, substituindo em 1.30 as expressões de $\phi_{1}^{\alpha}, \phi_{2}^{\alpha}$ e $\phi_{3}^{\alpha}$ dadas em 1.26 temos,

$$
\lambda^{2}\left|d z_{\alpha}\right|^{2}=\left(\frac{\left(1+|g|^{2}\right)|\eta|}{2}\right)^{2}
$$

Portanto a métrica de $X$ é dada por,

$$
d s^{2}=\frac{1}{4}\left(1+|g|^{2}\right)^{2}|\eta|^{2} \quad \text { ou } \quad d s^{2}=\lambda^{2}\left|d z_{\alpha}\right|^{2}, \quad \text { onde } \quad \lambda=\frac{1}{2}|f|\left(1+|g|^{2}\right)
$$

Por um simples cálculo se verifica que em coordenadas $z_{\alpha}$,

$$
X_{u} \times X_{v}=\operatorname{Im}\left\{\left(\phi_{2}^{\alpha} \overline{\phi_{3}^{\alpha}}, \phi_{3} \overline{\phi_{1}^{\alpha}}, \phi_{1} \overline{\phi_{2}^{\alpha}}\right)\right\} /\left|d z_{\alpha}\right|^{2}
$$

E como,

$$
\begin{array}{r}
\left\|X_{u} \times X_{v}\right\|^{2}=\lambda^{4}=\left[\frac{|f|\left(1+|g|^{2}\right)}{2}\right]^{4} \text { segue } \\
N(z)=\frac{X_{u} \times X_{v}}{\left\|X_{u} \times X_{v}\right\|}=\left(\frac{2 \operatorname{Re}\{g\}}{|g|^{2}+1}, \frac{2 \operatorname{Im}\{g\}}{|g|^{2}+1}, \frac{|g|^{2}-1}{|g|^{2}+1}\right)
\end{array}
$$

que é o vetor unitário normal a superfície $S$. Podemos visualizar $N$ dado em 1.32 como uma aplicação que vai de $M$ sobre a esfera unitária $S^{2}(1) \subset \mathbb{R}^{3}, M \longrightarrow S^{2}(1)$. Esta aplicação é chamada de aplicação normal de Gauss da superfície $S=X(M)$. Observemos que pelo exemplo (2) da seção 1.1 temos $g=\pi_{1} \circ N$. Logo $g$ é a representação analítica da aplicação normal de Gauss da imersão.

Proposição 1.5.9 A curvatura Gaussiana $K$ de uma superfície $S$, definida pela imersão conforme $X: M \longrightarrow X(M) \subset \mathbb{R}^{3}$ é dada localmente por:

$$
K=-\frac{\Delta \log \lambda}{\lambda^{2}}, \quad \text { onde } \quad \lambda^{2}=g_{i j} \delta_{i j}
$$


Demonstração: Basta usar a fórmula da curvatura Gaussiana em coordenadas ortogonais.

$$
K=\frac{-1}{2 \sqrt{g_{11} g_{22}}}\left\{\frac{\partial}{\partial u}\left(\frac{\left(g_{22}\right)_{u}}{\sqrt{g_{11} g_{22}}}\right)+\frac{\partial}{\partial v}\left(\frac{\left(g_{11}\right)_{v}}{\sqrt{g_{11} g_{22}}}\right)\right\}
$$

Proposição 1.5.10 Seja S uma superfície mínima definida pela imersão conforme $X: M \longrightarrow X(M) \subset \mathbb{R}^{3}$. Então usando a representação (1.26), a curvatura de Gauss em cada ponto é dada por

$$
K=-4 \frac{\left|\left\langle\frac{\partial}{\partial z} \Phi^{\alpha}, N\right\rangle\right|}{\left\|\Phi^{\alpha}\right\|^{4}} \quad \text { ou equivalentemente } \quad K=-\left[\frac{4\left|g^{\prime}\right|}{|f|\left(1+|g|^{2}\right)^{2}}\right]^{2}
$$

\section{Demonstração:}

Usando que $\Phi^{\alpha}=2 \frac{\partial}{\partial z} X d z_{\alpha}$, temos $\frac{\partial}{\partial z} \Phi^{\alpha}=\left(\frac{1}{2}\left(X_{u u}-X_{v v}\right)-i X_{u v}\right)\left(d z_{\alpha}\right)^{2}$

Como as coordenadas são isotérmicas segue do teorema 1.5 .8 que $\Phi^{\alpha}$ é dado pelas diferenciais holomorfas 1.26 o que implica $\Delta\left(x_{j} \circ z_{\alpha}^{-1}\right)=0$. Disto segue,

$$
\left|\left\langle\frac{\partial}{\partial z} \Phi^{\alpha}, . V\right\rangle\right|^{2}=\left(b_{11}^{2}+b_{12}^{2}\right)\left|d z_{\alpha}\right|^{4} \quad \text { e } \quad\left\|\Phi^{\alpha}\right\|^{4}=4 \lambda^{4}\left|d z_{\alpha}\right|^{4}
$$

Temos,

$$
\frac{-4\left|\left\langle\frac{\partial}{\partial z} \Phi^{\alpha}, N\right\rangle\right|^{2}}{\left\|\Phi^{\alpha}\right\|^{4}}=\frac{-4\left(b_{11}^{2}+b_{12}^{2}\right)\left|d z_{\alpha}\right|^{4}}{4 \lambda^{4}\left|d z_{\alpha}\right|^{4}}=\frac{-\left(b_{11}^{2}+b_{12}^{2}\right)}{\lambda^{4}}=K
$$

Por outro lado,

$\lambda^{4}=\frac{|f|^{4}\left(1+|g|^{2}\right)^{4}}{16}$ e $\left\langle\frac{\partial}{\partial z} \Phi^{\alpha}, N\right\rangle=-f g^{\prime}\left(d z_{\alpha}\right)^{2}, \quad \operatorname{logo} \quad K=-\left(\frac{4\left|g^{\prime}\right|}{|f|\left(1+|g|^{2}\right)^{2}}\right)^{2}$

Podemos agora dar uma interpretação geométrica da curvatura Gaussiana em termos de área sobre a esfera unitária através da aplicação normal de Gauss.

Definição 1.5.11 Sejam $S$ uma superfície definida pela imersão conforme $X: M \longrightarrow X(M) \subset \mathbb{R}^{3}$. Definimos a curvatura total de $S$ por:

$$
C(S):=\int_{M} K d M
$$

onde $d M$ é o elemento de área de $M$. 
Sejam $\left(U_{\alpha}, V_{\alpha}, \tilde{z}_{\alpha}\right)$ uma coordenada local de $M, D \subset V_{\alpha}$ uma região $C^{1}$ por partes de $\mathbb{C}$, a curvatura total de $S_{D}=X\left(z_{\alpha}^{-1}(D)\right)$ é $C\left(S_{D}\right)=\iint_{D} K \lambda^{2} d u d v$.

$$
\begin{aligned}
& \text { Como } K \lambda^{2}=-\left(\frac{2\left|g^{\prime}\right|}{\left(1+|g|^{2}\right)^{2}}\right)^{2} \text { e } \\
& \text { Área }\left(N\left(S_{D}\right)\right)=\iint_{D} d A=\iint_{D}\left\|N_{u} \times N_{v}\right\| d u d v=\iint_{D}\left(\frac{2\left|g^{\prime}\right|}{\left(1+|g|^{2}\right)^{2}}\right)^{2} d u d v,
\end{aligned}
$$

onde $d A$ é o elemento de área de $S^{2}(1)$. Então, $C\left(S_{D}\right)=-\operatorname{Área}\left(N_{D}\right)$.

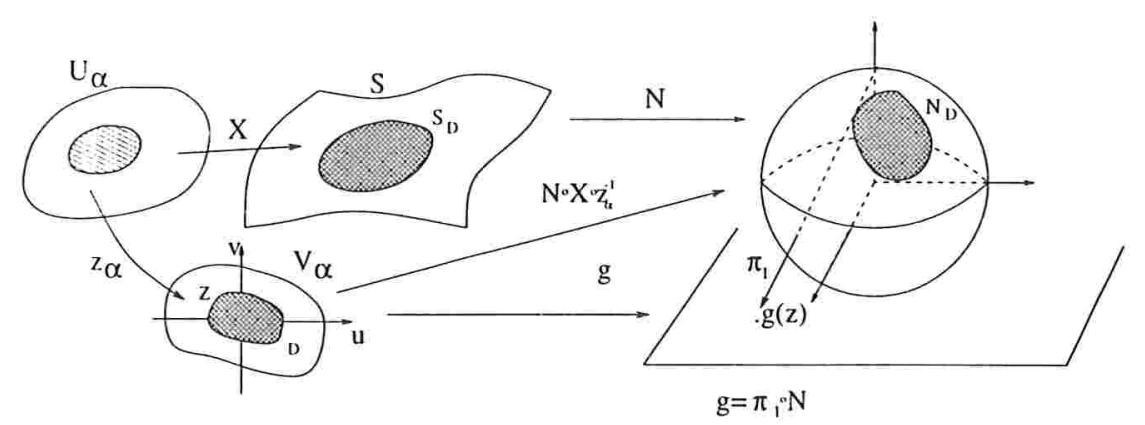

\section{Figura 1.17}

\subsubsection{Resultados Globais sobre Superfícies Mínimas}

Na subseção anterior estudamos algumas propriedades locais de imersão conforme. Agora resumiremos resultados que nos permitirão estudar superfícies mínimas globalmente, dando-nos uma ferramenta importante na construção de exemplos.

Definição 1.5.12 1) Um curva diferenciável $\gamma=X \circ \alpha:[0,1) \longrightarrow S$ é chamada divergente, se para qualquer subconjunto compacto $\mathcal{Q}$ de $M$, existe $t_{0} \in[0,1)$ tal que $\alpha(t) \notin \mathcal{Q}$ para $t>t_{0}$.

2) O comprimento da curva $\alpha:[0,1) \longrightarrow M$ é dada por:

$$
\operatorname{comp}(\alpha)=\lim _{t \rightarrow 1^{-}} \int_{0}^{t}\left\|\alpha^{\prime}(t)\right\| d t
$$


Definição 1.5.13 Uma superfície $S=X(M)$ é completa, se toda curva divergente de $M$, tem comprimento infinito.

A representação de Weierstrass local de acordo com 1.26 e 1.29, também podem ser definidas em termos globais sobre $M$. Já vimos que em 1.23

$$
\phi_{j}^{\alpha}(z)=2\left(\frac{\partial}{\partial z}\left(x_{j} \circ z_{\alpha}^{-1}\right)(z)\right) d z_{\alpha}, \quad j=1,2,3
$$

definem 1-formas diferenciais holomorfas globalmente em $M$, que chamaremos de $\phi_{j}(z), \quad j=1,2,3$,

No que segue,

$$
\Phi(z):=\left(\phi_{1}, \phi_{2}, \phi_{3}\right)=\left(\frac{1}{2}\left(1-g^{2}\right) \eta, \frac{i}{2}\left(1+g^{2}\right) \eta, g \eta\right)
$$

As demonstraçòes dos dois teoremas abaixo podem ser encontradas, por exemplo, em [19].

Teorema 1.5.14 Seja M uma variedade Riemanniana de dimensão 2 conexa, orientada, completa, com curvatura Gaussiana $K \leq 0$ e curvatura total $C(S)>-\infty$. Existe uma variedade compacta $\bar{M}$ de dimensão 2 , e um número finito de pontos $P_{1}, \ldots, P_{N}$ sobre $\bar{M}$, tais que $M$ é isométrica a $\bar{M} \backslash\left\{P_{1}, \ldots, P_{N}\right\}$.

Teorema 1.5.15 Seja $S$ uma superfície mínima regular completa, definida por $X: M \longrightarrow \mathbb{R}^{3}$. Se a curvatura total de $S$ é finita, então existe uma superfície de Riemann compacta $\bar{M}$, um número finito de pontos $P_{1}, \ldots, P_{N}$ de $\bar{M}$, tal que $M$ é conformemente equivalente à $\bar{M} \backslash\left\{P_{1}, P_{2}, \ldots, P_{N}\right\}$. Além disso a função $g: M \longrightarrow \mathbb{C}$ se estende meromorficamente sobre $\bar{M}$.

A função $g: \bar{M} \longrightarrow \mathbb{C} \cup\{\infty\}$ é holomorfa. Pelo teorema 1.1.6 $g$ é sobrejetora, e cada valor de $\mathbb{C} \cup\{\infty\}$ é assumido um número fixo de vezes, digamos $m$. Este número é o grau de $g$. 
Definição 1.5.16 Seja $M$ uma superfície de Riemann completa conformemente equivalente a uma superfície de Riemann compacta $\bar{M}$ de gênero $p$, menos um número finito de pontos $P_{1}, P_{2}, \ldots, P_{N}$. Dizemos que $M$ é de gênero $p$ com $N$ fins.

Sejam $F_{j}:=X\left(D_{j} \backslash\left\{P_{j}\right\}\right), j=1, \ldots, N$ os fins da imersão $X: \bar{M} \backslash\left\{P_{1}, \ldots, P_{N}\right\} \longrightarrow \mathbb{R}^{3}$ que define uma superfície completa $S=X(M)$, onde $D_{j} \backslash\left\{P_{j}\right\}$ é um disco topológico de $M$ perfurado em $P_{j}$, conforme a um disco perfurado de $\mathbb{C}$. Podemos dar um significado geométrico para as ordens $k_{\nu}$ dos fins de $S$. Nesse sentido, temos:

Teorema 1.5.17 Seja $X: M \longrightarrow \mathbb{R}^{3}$ uma imersão completa, onde $M$ é conformemente equivalente a uma superfície compacta menos um número finito de pontos,ou seja, $M$ é conformemente equivalente à $\bar{M} \backslash\left\{P_{1}, P_{2}, \ldots, P_{N}\right\}$. Se a aplicação normal de Gauss se extende continuamente à $\bar{M}$, temos:

(i) X é imersão própria.

(ii) Para $r$ suficientemente grande, $\frac{1}{r}\left(X(M) \cap S^{2}(r)\right) \subset S^{2}(1)$, onde $S^{2}(r)=\left\{x \in \mathbb{R}^{3} ;\|x\|=r\right\}$ consiste de $N$ curvas fechadas $\left\{\gamma_{1}^{r}, \gamma_{2}^{r}, \ldots, \gamma_{N}^{r}\right\}$ imersas em $S^{2}(1)$.

(iii) Quando $r \rightarrow \infty, \gamma_{j}^{r}$ converge $C^{1}$ à uma geodésica com multiplicidade em $S^{2}(1)$. (iv) Se X é uma imersão mínima, a convergência descrita no ítem (iii) é de classe $C^{\infty}$

Demonstração: ver [4] pag. 88 .

Proposição 1.5.18 Seja $S=X(M)$ uma superfície mínima completa em $\mathbb{R}^{3}$. Então a curvatura total de $S$ é dada por: $C(S)=-4 \pi \cdot \operatorname{grau}(g)$, ou $C(S)=-\infty$.

Teorema 1.5.19 (Jorge-Meeks-Gackstätter) Seja S uma superfície mínima completa do $\mathbb{R}^{3}$ com gênero $p$, curvatura total finita $C(S)$, definida pela imersão $X: \bar{M} \backslash\left\{P_{1}, \ldots, P_{N}\right\} \longrightarrow \mathbb{R}^{3}$. Sejam $F_{j}$ os fins de $X$, associados a $P_{j}, j=1,2, \ldots, N$ e $\Gamma_{r}^{j}=\frac{1}{r} F_{j} \cap S^{2}(r) \subset S^{2}(1)$, como no teorema 1.5 .17 , convergindo $C^{\infty}$ para a geodésica $\gamma_{j}$ de $S^{2}(1)$ com multiplicidade $k_{\nu}$, quando $r$ tende para o infinito. Então 
vale:

(i) $C(S)=2 \pi\left(\chi-\sum_{\nu=1}^{N} k_{\nu}\right) \quad$ ou equivalentemente $2 m=2 p-2+\sum_{\nu=1}^{N}\left(k_{\nu}+1\right)$, onde $m$ é o grau de $g$ e $\chi=2-2 p-N$.

ii) $F_{j}$ é mergulhado se, e somente se $k_{\nu}=1$. Isto é, $C(S)=2 \pi(\chi-N)$ se e só se os fins da imersão são mergulhados.

Demonstração: ver [13] ou [8]

Agora se $m_{\nu}, \nu=1, \ldots, N$ são as ordens dos polos de $\eta(z)=f(z) d z$ em $P_{j}$, temos a proposiçào:

Proposição 1.5.20 Seja $F_{j}$ um fim correspondente $\grave{a} P_{j}, j=1, \ldots, N$, da imersão $X$ de uma superfície mínima completa $S$ de curvatura total finita. Se $(g, \eta)$ é uma representaçào de Weierstrass de $S$, tal que $g\left(P_{j}\right)=0$, temos que $F_{j}$ é mergulhada se, e somente se $\eta$ tem um polo de ordem $2 \mathrm{em} P_{j}$. Em geral se $\eta$ tem um polo de ordem $m_{\nu} \geq 2 \mathrm{em} P_{j}$, então $k_{\nu}=m_{\nu}-1$.

Demonstração: ver [4]

O teorema que enunciaremos abaixo contém os passos fundamentais, os quais seguirei, para a demonstração de existência dos exemplos de superfícies mínimas contidas neste trabalho.

Teorema 1.5.21 (Teorema de Huber-Osserman) Sobre uma superfície de Riemann compacta $\bar{M}$ de gênero $p$, sejam g uma função meromorfa e $\eta$ uma diferencial meromorfa. Sejam $P_{1}, P_{2}, \ldots, P_{N}$ pontos de $\bar{M}$ e $M$ conformemente equivalente a $\bar{M} \backslash\left\{P_{1}, P_{2}, \ldots, P_{N}\right\}$.

Sejam $\phi_{1}, \phi_{2}, \phi_{3}$ diferenciais holomorfas sobre $M$ definidas por:

$$
\phi_{1}=\frac{1}{2}\left(1-g^{2}\right) \eta, \quad \phi_{2}=\frac{i}{2}\left(1+g^{2}\right) \eta, \quad \phi_{3}=g \eta
$$

e a função $X=\left(x_{1}, x_{2}, x_{3}\right): M \longrightarrow \mathbb{R}^{3}$, onde

$$
x_{j}=\operatorname{Re}\left\{\int_{z_{0}}^{z} \phi_{j}\right\}, j=1,2,3, z_{0}, z \in M, z_{0} \text { fixado } e
$$




$$
X(z)=\left(\operatorname{Re}\left\{\int_{z_{0}}^{z} \frac{1}{2}\left(1-g^{2}\right) \eta\right\}, \operatorname{Re}\left\{\int_{z_{0}}^{z} \frac{i}{2}\left(1+g^{2}\right) \eta\right\}, \operatorname{Re}\left\{\int_{z_{0}}^{z} g \eta\right\}\right)
$$

Então, se as condições $\mathbf{c}_{1}, \mathbf{c}_{2}, \mathbf{c}_{3}$ e $\mathbf{c}_{4}$ abaixo são verificadas, $X$ é uma imersão mínima completa em $\mathbb{R}^{3}$ de uma superfície de gênero $p$ e $N$ fins com curvatura total finita. Sob estas condições o par $(g, \eta)$ é chamado representação de Weierstrass da imersão.

( $\left.\mathbf{c}_{1}\right) \eta$ é holomorfa em $M$. Os zeros de $\eta \mathrm{em} M$ coincidem com os polos de $\mathrm{g} \mathrm{em}$ $M$. Além disso. $P \in M$ é um polo de ordem $m$ de $g$ se e somente se $P$ é um zero de ordem $2 m$ de $\eta$.

(c) Se a é uma curva fechada em $M$ e não trivial na homologia de $\bar{M}$, então $\operatorname{Re}\left\{\int_{\alpha} \phi_{j}\right\}=0, \forall j=1,2,3$.

$\left(\mathrm{c}_{3}\right)$ Os resíduos de $\phi_{j}$ em $P_{k}$ são reais, isto é,

$\operatorname{Res}\left(\phi_{j}, P_{k}\right) \in \mathbb{R} \quad \forall j=1,2,3$ e $k=1,2, \ldots, N$. Ou equivalentemente,

$$
\operatorname{Res}\left(g \eta ; P_{k}\right) \in \mathbb{R} \text { e } \overline{\operatorname{Res}\left(\eta, P_{k}\right)}+\operatorname{Res}\left(g^{2} \eta ; P_{k}\right)=0
$$

( $\left.\mathbf{c}_{4}\right)$ Toda curva divergente $\gamma$ em M possui comprimento infinito. Como a métrica é:

$$
\begin{gathered}
d s^{2}=\left[\frac{1}{2}\left(1+|g|^{2}\right)|\eta|\right]^{2}, \quad \text { se } \eta=f d z \text { temos } \\
L(\gamma)=\int_{\gamma} d s=\frac{1}{2} \int_{\gamma}|f|\left(1+|g|^{2}\right)|d z|=\infty .
\end{gathered}
$$




\section{Capítulo 2}

\section{Superfície Mínima de Chen-Gackstätter de Gênero 1}

Neste capítulo será demonstrado que existe uma superfície mínima completa do $\mathbb{R}^{3}$ com gênero um e curvatura total finita $-8 \pi$. Na primeira seção exporemos resultados importantes, usados no decorrer na demonstração do teorema principal do capítulo, enunciado na segunda seção.

\subsection{Fórmulas de $\wp$ e $\zeta$ no quadrado $[0,1,1+i, i]$}

Lema 2.1.1 No paralelogramo fundamental periódico $F$ mais simétrico possível o quadrado de vértices $[0,1,1+i, i]$, as funções $\wp(z)$ e $\zeta(z)$ são dadas explicitamente por:

$$
\wp(z)=\frac{1}{z^{2}}+\sum_{\substack{(m, n) \in \mathbb{Z}^{2} \\(m, n) \neq(0,0)}}\left\{\frac{1}{(z-m-n i)^{2}}-\frac{1}{(m+n i)^{2}}\right\}
$$

$e$

$$
\zeta(z)=\frac{1}{z}+\sum_{\substack{(m, n) \in \mathbf{Z}^{2} \\(m, n) \neq(0,0)}}\left\{\frac{1}{(z-m-n i)}+\frac{1}{(m+n i)}+\frac{z}{(m+n i)^{2}}\right\}
$$

Valem as seguintes igualdades: 
(i) $\wp(1 / 2)+\wp(i / 2)=e_{1}+e_{3}=0 \Rightarrow e_{1}=-e_{3} \in \mathbb{R}$

(ii) $e_{2}=g_{3}=0$

(iii) $g_{2}=4 e_{1}^{2}>0$

(iv) $\wp^{\prime}(z)^{2}=4 \wp(z)\left(\wp^{2}(z)-e_{1}^{2}\right)$

$(v) \wp^{\prime \prime}(z)=6 \wp^{2}(z)-\frac{1}{2} g_{2}$.

\section{Demonstração:}

Para provar o ítem $(i)$, vamos calcular $\wp(1 / 2)$ e $\wp(i / 2)$. Primeiro

$$
\begin{aligned}
\rho(1 / 2) & =\frac{1}{(1 / 2)^{2}}+\sum_{w \neq 0}\left\{\frac{1}{(1 / 2-m-n i)^{2}}-\frac{1}{(m+n i)^{2}}\right\} \\
& =4+\sum_{w \neq 0}\left\{\frac{4}{(1-2(m+n i))^{2}}-\frac{1}{(m+n i)^{2}}\right\}
\end{aligned}
$$

Agora,

$$
\begin{aligned}
\rho(i / 2) & =\frac{1}{(i / 2)^{2}}+\sum_{w \neq 0}\left\{\frac{1}{(i / 2-(m+n i))^{2}}-\frac{1}{(m+n i)^{2}}\right\} \\
& =-4+\sum_{w \neq 0}\left\{\frac{4}{(i-2(m+n i))^{2}}-\frac{1}{(m+n i)^{2}}\right\} \\
& =-4+\sum_{w \neq 0}\left\{\frac{4}{i^{2}(1-2(-i m+n))^{2}}-\frac{1}{i^{2}(-i m+n)^{2}}\right\} \\
& =-4+\sum_{w \neq 0}\left\{\frac{-4}{(1-2(-i m+n))^{2}}+\frac{1}{(-i m+n)^{2}}\right\} \\
& =-\left(4+\sum_{w \neq 0}\left\{\frac{1}{(1-2(-i m+n))^{2}}-\frac{1}{(-i m+n)^{2}}\right\}\right)
\end{aligned}
$$

cuja soma que aqui aparece, difere da soma de $\wp(1 / 2)$ somente na ordem de seus termos. Como estas séries são absolutemente convergentes, fazendo um rearranjo dos termos, $w^{\prime}=-i m+n$ para $w=m+i n$, não alteramos o valor da soma.

Segue que $\wp(i / 2)=-\wp(1 / 2), \log \wp \wp(1 / 2)+\wp(i / 2)=e_{1}+e_{3}=0$. 
(ii) Pelo ítem (i) do corolário 1.4.13, $e_{1}+e_{2}+e_{3}=0$, então usando $(i)$ temos $e_{2}=0$. Como $g_{3} / 4=e_{1} \cdot e_{2} \cdot \epsilon_{3}$. então $g_{3}=0$.

(iii) Do ítem (ii) do corolário 1.4.13, temos $e_{1} \cdot e_{2}+e_{2} \cdot e_{3}+e_{1} \cdot e_{3}=-g_{2} / 4$, usando (i), $e_{3}=-e_{1},-e_{1}^{2}=-g_{2} / 4 \Rightarrow g_{2}=4 e_{1}^{2}>0$.

(iv) Vimos que

$$
\begin{aligned}
\wp^{\prime 2}(z) & =4\left(\wp(z)-e_{1}\right)\left(\wp(z)-e_{2}\right)\left(\wp(z)-e_{3}\right) \\
& =4\left(\wp(z)-e_{1}\right)\left(\wp(z)-e_{3}\right) \wp(z) \\
& =4\left(\wp(z)-e_{1}\right)\left(\wp(z)+e_{1}\right) \wp(z)=4\left(\rho^{2}(z)-e_{1}{ }^{2}\right) \wp(z) \\
& =4 \wp(z)\left(\wp^{2}(z)-g_{2} / 4\right)=\wp(z)\left(4 \wp^{2}(z)-g_{2}\right)
\end{aligned}
$$

(v) De 1.13 , temos

$$
\rho^{\prime \prime}(z)=\frac{6}{z^{4}}+6 G_{4}+60 G_{6} z^{2}+\ldots
$$

e de 1.12 , segue

$$
\wp(z)=\frac{1}{z^{2}}+3 G_{4} z^{2}+5 G_{6} z^{4}+\ldots
$$

Logo,

$$
6 \jmath^{2}-\frac{1}{2} g_{2}=\frac{6}{z^{4}}+6 G_{4}+60 G_{6} z^{2}+\ldots
$$

Lema 2.1.2 Para todo $z \in F$ como no lema 2.1.1, temos:

(i) $\wp\left(z-\frac{1}{2}\right)-\wp\left(z-\frac{i}{2}\right)-2 . e_{1}=16 \cdot e_{1}^{3} \cdot \frac{\wp(z)}{\varrho^{\prime}(z)}$

(ii) $i \zeta(i z)=\zeta(z)$

(iii) $\zeta(1 / 2)=i \zeta(i / 2)=\pi / 2$

(iv) $\zeta\left(\frac{1+i}{2}\right)=\frac{(1-i) \pi}{2}$.

\section{Demonstração:}

Para provar $(i)$, usaremos a fórmula da adição para $\wp(z)$,

$$
\wp\left(z+z_{0}\right)+\wp(z)+\wp\left(z_{0}\right)=\frac{1}{4}\left[\frac{\wp^{\prime}(z)-\wp^{\prime}\left(z_{0}\right)}{\wp(z)-\wp\left(z_{0}\right)}\right]^{2} .
$$


Substituindo $z_{0}=-1 / 2$ em 2.1 , temos

$$
\begin{gathered}
\wp\left(z-\frac{1}{2}\right)+\wp(z)+\wp\left(-\frac{1}{2}\right)=\frac{1}{4}\left[\frac{\wp^{\prime}(z)-\wp^{\prime}\left(-\frac{1}{2}\right)}{\wp(z)-\wp\left(-\frac{1}{2}\right)}\right]^{2} . \\
\wp\left(z-\frac{1}{2}\right)+\wp(z)+e_{1}=\frac{1}{4}\left[\frac{\wp^{\prime}(z)}{\wp(z)-e_{1}}\right]^{2} \\
\wp\left(z-\frac{1}{2}\right)+\wp(z)+e_{1}=\frac{\wp^{\prime 2}(z)}{4\left(\wp(z)-e_{1}\right)^{2}}
\end{gathered}
$$

Pelo ítem (iv) do Lema 2.1.1, temos

$$
\begin{aligned}
& \wp\left(z-\frac{1}{2}\right)+\wp(z)+e_{1}=4 \wp(z) \frac{\left(\wp^{2}(z)-e_{1}^{2}\right)}{4\left(\wp(z)-e_{1}\right)^{2}} \\
& \wp\left(z-\frac{1}{2}\right)+\wp(z)+e_{1}=\wp(z) \frac{\wp(z)+e_{1}}{\wp(z)-e_{1}}
\end{aligned}
$$

Donde segue,

$$
\wp\left(z-\frac{1}{2}\right)=\frac{\wp(z) e_{1}+e_{1}^{2}}{\wp(z)-e_{1}}
$$

Agora substituindo $z_{0}=-i / 2$ em 2.1,

$$
\begin{aligned}
& \wp(z-i / 2)=\frac{1}{4}\left[\frac{\wp^{\prime}(z)-\wp^{\prime}(-i / 2)}{\wp(z)-\wp(-i / 2)}\right]^{2}-\wp(z)-\wp(-i / 2) \\
&=\frac{\wp^{\prime}(z)^{2}}{4\left(\wp(z)-e_{3}\right)^{2}}-\wp(z)-e_{3} \\
&=\frac{4 \wp(z)\left(\wp(z)+e_{1}\right)\left(\wp(z)-e_{1}\right)}{4\left(\wp(z)+e_{1}\right)^{2}}-\left(\wp(z)+e_{3}\right) \\
&=\frac{\wp(z)\left(\wp(z)-e_{1}\right)-\left(\wp^{2}(z)-e_{1}^{2}\right)}{\wp(z)+e_{1}} \\
&= \frac{\wp^{2}(z)-\wp(z) e_{1}-\wp^{2}(z)+e_{1}^{2}}{\wp(z)+e_{1}}=\frac{e_{1}{ }^{2}-\wp(z) e_{1}}{\wp(z)+e_{1}} \\
& \wp\left(z-\frac{i}{2}\right)=\frac{e_{1}{ }^{2}-\wp(z) e_{1}}{\wp(z)+e_{1}}
\end{aligned}
$$

Então, de 2.2 e 2.3 , temos

$$
\wp\left(z-\frac{1}{2}\right)-\wp\left(z-\frac{i}{2}\right)-2 e_{1}=\frac{\wp(z) e_{1}+e_{1}{ }^{2}}{\wp(z)-e_{1}}+\frac{\wp(z) e_{1}-e_{1}{ }^{2}}{\wp(z)+e_{1}}-2 e_{1}
$$




$$
\begin{aligned}
& =\frac{e_{1}\left(\wp(z)+e_{1}\right)^{2}+e_{1}\left(\wp(z)-e_{1}\right)^{2}-2 e_{1}\left(\wp^{2}(z)-e_{1}{ }^{2}\right)}{\left(\wp(z)-e_{1}\right)\left(\wp(z)+e_{1}\right)} \\
& =\frac{2 e_{1} \wp^{2}(z)+2 e_{1}{ }^{3}-2 e_{1} \wp^{2}(z)+2 e_{1}{ }^{3}}{\wp^{2}(z)-e_{1}{ }^{2}}=\frac{4 e_{1}{ }^{3}}{\wp^{2}(z)-e_{1}{ }^{2}} \\
& =\frac{4 e_{1}{ }^{3}}{\frac{\wp^{\prime}(z)}{4 \wp(z)}}=16 e_{1}{ }^{3} \frac{\wp(z)}{\wp^{\prime 2}(z)}
\end{aligned}
$$

(ii) De

$$
\zeta(z)=\frac{1}{z}+\sum_{w \neq 0}\left\{\frac{1}{z-w}+\frac{1}{w}+\frac{z}{w^{2}}\right\}
$$

Obtemos,

$$
\begin{gathered}
\zeta(i z)=\frac{1}{i z}+\sum_{w \neq 0}\left\{\frac{1}{i z-w}+\frac{1}{w}+\frac{i z}{w^{2}}\right\} \\
\zeta(i z)=\frac{-i}{z}+\sum_{w \neq 0}\left\{\frac{-i}{z-(-i w)}-\frac{i}{-i w}+\frac{(-i)^{2} \cdot i z}{(-i)^{2} \cdot w^{2}}\right\} \\
\zeta(i z)=\frac{-i}{z}+\sum_{w \neq 0}\left\{\frac{-i}{z-(-i w)}-\frac{i}{-i w}+\frac{-i z}{(-i w)^{2}}\right\} \\
\zeta(i z)=-i\left(\frac{1}{z}+\sum_{w \neq 0}\left\{\frac{1}{z-(-i w)}+\frac{1}{-i w}+\frac{z}{(-i w)^{2}}\right\}\right)
\end{gathered}
$$

Por um rearranjo nos termos da soma em $-i w$ para $w$, temos que $\zeta(i z)=-i \zeta(z)$, $\log i \zeta(i z)=\zeta(z)$.

(iii) Como $\eta_{1}=\zeta\left(w_{1} / 2\right)$ e $\eta_{2}=\zeta\left(w_{2} / 2\right)$, fazendo $z=1 / 2$ em (ii), $i \zeta(i / 2)=\zeta(1 / 2)$ e sabendo que $\eta_{1} w_{2}-\eta_{2} w_{1}=\pi i$, e como $w_{1}=1$ e $w_{2}=i$, temos que

$\zeta\left(\frac{1}{2}\right) \cdot i-\zeta\left(\frac{i}{2}\right) \cdot 1=\pi i \Rightarrow i^{2} \zeta\left(\frac{i}{2}\right)-\zeta\left(\frac{i}{2}\right)=\pi i \Rightarrow-2 \zeta\left(\frac{i}{2}\right)=\pi i \Rightarrow i \zeta\left(\frac{i}{2}\right)=\frac{\pi}{2}$

Portanto,

$$
\zeta\left(\frac{1}{2}\right)=i \zeta\left(\frac{i}{2}\right)=\frac{\pi}{2}
$$

(iv) Como,

$$
\zeta\left(z+\frac{w_{1}}{2}\right)=\zeta\left(z-\frac{w_{1}}{2}\right)+2 \zeta\left(\frac{w_{1}}{2}\right) \quad \mathrm{e}
$$




$$
\zeta\left(z+\frac{w_{2}}{2}\right)=\zeta\left(z-\frac{w_{2}}{2}\right)+2 \zeta\left(\frac{w_{2}}{2}\right) .
$$

Pondo, $z=\frac{w_{2}}{2}$ e $z=\frac{w_{1}}{2}$, respectivamente, na primeira e segunda equação acima. Temos:

$$
\zeta\left(\frac{w_{2}}{2}+\frac{w_{1}}{2}\right)=\zeta\left(\frac{w_{2}}{2}\right)+\zeta\left(\frac{w_{1}}{2}\right) \Rightarrow \zeta\left(\frac{1+i}{2}\right)=\zeta\left(\frac{1}{2}\right)+\zeta\left(\frac{i}{2}\right)=\frac{(1-i) \pi}{2} .
$$

\subsection{Exemplo de Chen-Gackstätter de gênero 1}

Do teorema 1.1.4 segue que qualquer superfície de Riemann compacta de gênero 1 , pode ser representada, a menos de equivalência conforme, por $\mathbb{C} / L(1, \tau)$ onde $\tau \in F M$ que denotaremos por $\bar{R}_{(1, \tau)}$. Quando $\tau=i$, o paralelogramo fundamental, $F$, é simplesmente o quadrado de vértices $[0,1,1+i, i]$, portanto, a superfície de Riemann mais simétrica de gênero 1 é $\bar{R}_{(1, i)}$.

A idéia da construção que será feita no teorema abaixo, consiste em construir uma superfície mínima $S$, completa, com curvatura total finita, conformemente equivalente a uma superfície de Riemann compacta menos um ponto, $\bar{R}_{(1, i)} \backslash\{0\}$. Para tal usaremos a teoria das funções elípticas abordada no capítulo 1 e o teorema 1.5.21.

Quando o valor absoluto da curvatura total de uma superfície é grande, sua geometria se torna difícil. Logo, é interessante construir exemplos com $|C(S)|$ tão pequeno quanto possível para gênero $p$ fixo.

A fórmula,

$$
C(S)=2 \pi\left(2-2 p-N-\sum_{\nu=1}^{N} k_{\nu}\right)
$$

do teorema 1.5.19, nos dá algumas informações: 


\begin{tabular}{|c|c|c|c|c|c|}
\hline$C(S)$ & $p$ & $N$ & $k_{\nu}$ & Classificação & Existência \\
\hline \multirow{2}{*}{$-4 \pi$} & \multirow{2}{*}{0} & 1 & $k_{1}=3$ & superfície de Enneper & \multirow{2}{*}{ somente essas } \\
\hline & & 2 & $k_{1}=k_{2}=1$ & Catenóide & \\
\hline \multirow{2}{*}{$-8 \pi$} & \multirow{2}{*}{1} & 1 & $k_{1}=3$ & tipo Enneper com uma alça & existe \\
\hline & & 2 & $k_{1}=k_{2}=1$ & tipo Catenóide com uma alça & não existe \\
\hline \multirow{2}{*}{$-12 \pi$} & \multirow{2}{*}{2} & 1 & $k_{1}=3$ & tipo Enneper com duas alças & existe \\
\hline & & 2 & $k_{1}=k_{2}=1$ & tipo Catenóide com duas alças & não existe \\
\hline$\vdots$ & $\vdots$ & & $\vdots$ & $\vdots$ & \\
\hline \multirow{2}{*}{$-4(p+1) \pi$} & \multirow{2}{*}{$p$} & 1 & $k_{1}=3$ & tipo Enneper com $p$ alças & existe \\
\hline & & 2 & $k_{1}=k_{2}=1$ & tipo Catenóide com $p$ alças & não existe \\
\hline
\end{tabular}

\section{Observações:}

(1) Osserman em [19], classificou as superfícies, Catenóide e Enneper, como as únicas superfícies mínimas completas orientadas de curvatura total $-4 \pi$.

(2) Em [16], Francisco J. López classificou totalmente as superfícies mínimas completas orientadas, com curvatura total $-8 \pi$. Mais precisamente se $S$ tem as propriedades acima, então $S$ é uma das seguintes superfícies: Um plano, um Catenóide, a superfície de Enneper, as superfícies descritas em [16] nos teoremas 3, 4, 5 e 6 (superfícies mínimas de gênero 0 e curvatura total $-8 \pi$ ) ou a Superfície de ChenGackstätter dada no teorema 2.2.1 abaixo.

(3) Ainda em [16], López provou que a superfície de Chen-Gackstätter dada no teorema 2.2.1 abaixo, é a única superfície mínima completa, orientada, de gênero 1 e com curvatura total $-8 \pi$.

(4) No capítulo 3 deste trabalho, trataremos do segundo exemplo de Chen-Gackstätter, que é uma superfície mínima de gênero 2, completa, orientada com curvatura total $-12 \pi$, com um fim do tipo Enneper. López, Martín e Rodríguez em [17] provaram que esta superfície é única.

(5) Richard M. Schoen, provou em [22] nas proposições 1 e teorema 3, que se $S$ é uma superfície mínima completa do $\mathbb{R}^{3}$, com curvatura total finita e com 2 fins mergulhados, então $S$ é o catenóide, ou um par de planos paralelos. 
(6) É possível construir um exemplo do tipo Enneper com mais de duas alças, imergindo em $\mathbb{R}^{3}$ uma superfície de Riemann hiperelíptica menos um ponto?.

No caso $p=3$ a resposta é afirmativa e a solução do problema se encontra em [6], e para $p>3$ ver [25].

(7) Seria interessante construir uma superfície mínima completa mergulhada de gênero 1.

Celso Costa mostrou em [3] que existe uma constante $C=2 e_{1} \sqrt{2 \pi}$ tal que a escolha para o par $(g, \eta)$, com $g=\frac{C}{\wp^{\prime}}$ e $\eta=\wp d z$ é a representação de Weierstrass de uma superfícies mínima completa $S$ de gênero 1 com curvatura total $C(S)=-12 \pi \mathrm{em}$ $\mathbb{R}^{3}$, com três fins mergulhados. Hoffman e Meeks em [12] deram uma prova analítica de que tal superfície é mergulhada .

Teorema 2.2.1 (Chen-Gackstätter 1) Existe uma superfície mínima completa $S=X\left(R_{(1, i)}\right)$ com gênero $p=1$, curvatura total finita $C(S)=-8 \pi$, um fim de ordem 3, a qual é uma superfície de tipo Enneper com uma alça, onde:

$$
\left\{\begin{array}{l}
R_{(1, i)}=\bar{R}_{(1, i)} \backslash\{0\} \\
g(z)=A \frac{\wp^{\prime}(z)}{\wp(z)}, \quad A=\sqrt{\frac{3 \pi}{2 g_{2}}} \\
\eta(z)=2 \wp(z) d z
\end{array}\right.
$$

\section{Demonstração:}

Precisamos provar que o par $(g, \eta)$ é a representação de Weierstrass de uma imersão,

$$
X=\left(x_{1}, x_{2}, x_{3}\right): R_{(1, i)}=\bar{R}_{(1, i)} \backslash\{0\} \longrightarrow \mathbb{R}^{3}
$$

com as propriedades exigidas. Para isso mostraremos que o par $(g, \eta)$ satisfaz as condições $\mathbf{c}_{1}, \mathrm{c}_{2}, \mathrm{c}_{3}$ e $\mathrm{c}_{4}$ do Teorema 1.5.21

\section{Demonstração de $c_{1}$ :}

Pela definição de $\wp(z)$ já vimos que seus polos de ordem 2 pertencem ao reticulado $L(1, i)$. Porém, em $R_{(1, i)}=\bar{R}_{(1, i)} \backslash\{0\}, \wp(z)$ é holomorfa em todos os pontos, pois 
neste caso estão fora as singularidades: $0,1,1+i, i$. Logo, $\eta=2 \wp(z) d z$ é holomorfa em $R_{(1, i)}$. Veja figura 2.1

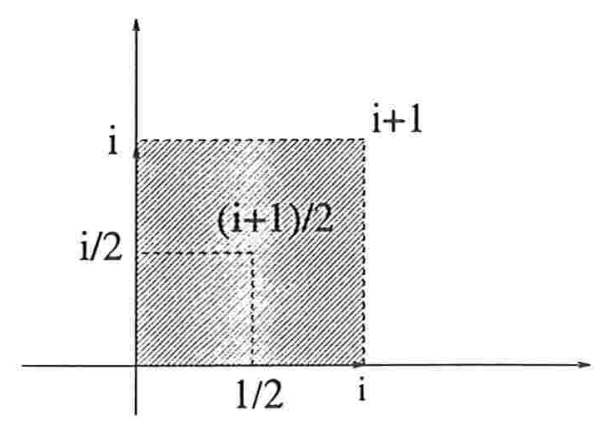

Figura 2.1

Agora do lema (2.1.1) item (ii), temos que $\wp\left(\frac{1+i}{2}\right)=e_{2}=0$, e então $z_{0}=(1+i) / 2$ é um zero duplo de $\rho$, já que $\wp^{\prime}\left(\frac{1+i}{2}\right)=e_{2}=0$. Como a soma das ordens dos zeros menos a soma das ordens dos polos é igual a zero, e o único polo em $\bar{R}_{(1, i)} \backslash\{0\}$ é o zero, concluímos que $z_{0}=(1+i) / 2$ é o único zero duplo de $\eta=2 \wp(z) d z$ em $R_{(1, i)}$. Por outro lado, $g=A \frac{\rho^{\prime}(z)}{\rho(z)}$ é uma função elíptica em $L(1, i)$ e tem um polo simples em $R_{(1, i)}$, pois em $z_{0}=(1+i) / 2, \wp^{\prime}$ assume um zero simples e $\wp$ um zero duplo. Portanto $z_{0}$ é um polo simples de $g$. Segue que $c_{1}$ é satisfeita.

Antes de mostrar $c_{2}$, observemos a seguinte tabela:

\begin{tabular}{|c|c|c|c|c|}
\hline$z$ & 0 & $\frac{1+i}{2}$ & $\frac{1}{2}$ & $\frac{i}{2}$ \\
\hline$\eta=2 \wp(z) d z$ & $\infty^{2}$ & $0^{2}$ & $2 e_{1}$ & $-2 e_{1}$ \\
\hline$g=A \frac{\rho^{\prime}}{\rho}$ & $\infty$ & $\infty$ & 0 & 0 \\
\hline $\mathrm{S}$ & $\begin{array}{c}\text { fim de ordem } \\
k_{1}=3\end{array}$ & $\begin{array}{c}\text { ponto } \\
\text { regular }\end{array}$ & $\begin{array}{c}\text { ponto } \\
\text { regular }\end{array}$ & $\begin{array}{c}\text { ponto } \\
\text { regular }\end{array}$ \\
\hline
\end{tabular}

Em $z=0, \wp$ tem um polo de ordem 2, então $\eta$ tem um polo de ordem 2 e $g$ tem um polo simples, pois $\wp^{\prime}$ tem um polo de ordem 3 . E é fácil ver que $g$ e $\eta$ tem os valores dados na quarta e quinta coluna da tabela.

Observamos que a função meromorfa $g: R_{(1, i)} \longrightarrow \mathbb{C}$, estende-se holomorficamente para $g: \bar{R}_{(1, i)} \longrightarrow \overline{\mathbb{C}}_{w}$, e o seu grau é dois 2 . Para provar isto basta usar 
o fato de que toda função elíptica de grau 2, assume qualquer valor duas vezes no paralelogramo fundamental e que o grau de uma função elíptica é a soma das ordens de seus polos (ou zeros) em $\bar{R}_{(1, i)}$.

Observe na tabela acima, que os polos simples de $g$ são $z=0$ e $z=\frac{1+i}{2}$. Logo $\operatorname{grau}(g)=2$ e $\mathrm{C}(\mathrm{S})=-4 \pi \cdot \operatorname{grau}(g)=-8 \pi$.

Da fórmula de Jorge-Meeks,

$$
C(S)=2 \pi\left(\chi-\sum_{\nu=1}^{N} k_{\nu}\right)
$$

para $N=1, p=1$ e $\gamma=2-2 p-N=-1$, temos

$$
-8 \pi=2 \pi\left(-1-k_{1}\right) \text { e então, } k_{1}=3 .
$$

Logo o fim nào é mergulhado.

\section{Demonstração de $c_{2}$ :}

Precisamos mostrar que se $\alpha:[0,1] \longrightarrow \mathbb{C}$ é uma curva fechada em $R_{(1, i)}$ e não trivial na homologia de $\bar{R}_{(1, i)}$ (i.e. $\alpha$ não é homotópica a zero), então $\operatorname{Re}\left\{\int_{\alpha} \phi_{j}\right\}=0$, $\forall j=1,2,3$.

Usando as fórmulas de Weierstrass;

$$
\left\{\begin{array}{l}
\phi_{1}=\frac{1}{2}\left(1-g^{2}\right) \eta \\
\phi_{2}=\frac{i}{2}\left(1+g^{2}\right) \eta \\
\phi_{3}=g \eta
\end{array}\right.
$$

$\operatorname{com} \eta=2 \wp d z$ e $g=A \frac{\varsigma^{\prime}}{\wp}$, podemos expressar $\phi_{1}, \phi_{1}, \phi_{3}$ em termos de $\wp, \wp^{\prime}, \wp^{\prime \prime}$ e $g_{2}$.

$$
\phi_{1}=\frac{1}{2} 2 \wp\left(1-\left(A \frac{\wp^{\prime}}{\wp}\right)^{2}\right) d z=\wp\left(1-A^{2} \frac{\wp^{\prime 2}}{\wp^{2}}\right) d z
$$

Pelos ítems $(i v)$ e $(v)$ do lema (2.1.1), $\wp^{2}=4 \wp\left(\wp^{2}-e_{1}^{2}\right), \wp^{\prime \prime}=6 \wp^{2}-\frac{1}{2} g_{2}$.

$$
\begin{aligned}
& \phi_{1}=\wp\left(1-A^{2} \frac{4 \wp\left(\wp^{2}-e_{1}^{2}\right)}{\wp^{2}}\right) d z=\wp\left(1-4 A^{2} \frac{\wp^{2}-e_{1}^{2}}{\wp}\right) d z \\
& \phi_{1}=\left(\wp-4 A^{2}\left(\wp^{2}-e_{1}^{2}\right)\right) d z, \quad \text { mas } \wp^{2}=\frac{\wp^{\prime \prime}}{6}+\frac{1}{12} g_{2} \quad \text { e } \quad e_{1}{ }^{2}=\frac{1}{4} g_{2}
\end{aligned}
$$




$$
\begin{aligned}
& \phi_{1}=\left(\wp-4 A^{2}\left(\frac{\rho^{\prime \prime}}{6}+\frac{1}{12} g_{2}-\frac{1}{4} g_{2}\right)\right) d z=\left(\wp-\frac{2}{3} A^{2} \wp^{\prime \prime}+\frac{2}{3} A^{2} g_{2}\right) d z \\
& \phi_{2}=\frac{i}{2} 2 \wp\left(1+A^{2} \frac{\rho^{\prime 2}}{\wp^{2}}\right) d z=i \wp\left(1+A^{2} 4 \frac{\wp\left(\wp^{2}-\frac{1}{4} g_{2}\right)}{\wp^{2}}\right) d z \\
& \phi_{2}=\left(i \rho+i A^{2} 4\left(\wp^{2}-\frac{1}{4} g_{2}\right)\right) d z=\left(i \wp+i A^{2} 4\left(\frac{\rho^{\prime \prime}}{6}+\frac{1}{12} g_{2}-\frac{1}{4} g_{2}\right)\right) d z \\
& \phi_{2}=\left(i \rho+4 i A^{2} \frac{\rho^{\prime \prime}}{6}-i \frac{8}{12} g_{2} A^{2}\right) d z=\left(i \wp+\frac{2 i}{3} A^{2} \rho^{\prime \prime}-\frac{2 i}{3} A^{2} g_{2}\right) d z \\
& \phi_{3}=g \eta=2 \wp A \frac{\rho^{\prime}}{\rho^{\prime}} d z=2 A \wp^{\prime} d z
\end{aligned}
$$

Sejam a. 3: $[0.1] \longrightarrow \mathbb{C}$ curvas, definidas por: $\alpha(t)=\frac{i}{2}+t, \quad t \in[0,1] \mathrm{e}$ $\beta(t)=\frac{1}{2}+i t, t \in[0.1]$, cujas imagens pela projeção estão em $\bar{R}_{(1, i)}$. São duas curvas fechadas em $R_{(1, i)}$ e nào triviais na homologia de $\bar{R}_{(1, i)}$ veja figura 2.2 . Já que $\bar{R}_{(1, i)}$ é homeomorfo a $S^{1} \times S^{1}$ e como o grupo fundamental $\pi_{1}\left(S^{1} \times S^{1}\right)=\pi_{1}\left(S^{1}\right) \oplus \pi_{1}\left(S^{1}\right)=$ $\mathbb{Z} \oplus \mathbb{Z}$, temos $\pi_{1}\left(\bar{R}_{(1, i)}\right)=\mathbb{Z} \oplus \mathbb{Z}$, pois $\pi_{1}$ é um invariante topológico. Além disso, $H_{1}\left(\bar{R}_{(1, i)}\right)=\pi_{1}\left(\bar{R}_{(1, i)}\right) /[$,$] , onde [,] é o comutador de \pi_{1}$ que neste caso é abeliano. Logo, $H_{1}\left(\bar{R}_{(1, i)}\right)=\pi_{1}\left(\bar{R}_{(1, i)}\right)=\mathbb{Z} \oplus \mathbb{Z}$. Portanto, toda curva fechada $\gamma$ em $\mathbb{R}_{(1, i)}$ é gerado por $\{\alpha, 3\}$. Então, o período de $\gamma$ fica completamente determinado calculando os períodos de $\alpha$ e $\beta$. pois

$$
\int_{\gamma} \phi_{j}(z)=m \int_{\alpha} \phi_{j}(z)+n \int_{\beta} \phi_{j}(z), \text { onde } j=1,2,3 \text { e } m, n \in \mathbb{Z}
$$
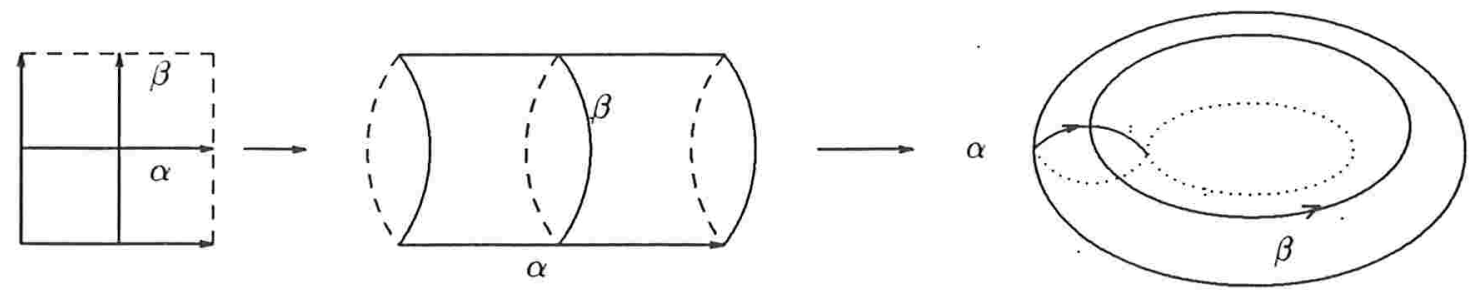

\section{Figura 2.2}


Vamos calcular agora o primeiro perído de $\phi_{1}$ em $R_{(1, i)}$

$$
\begin{aligned}
\int_{\alpha} \phi_{1}(z) & =\int_{\alpha}\left(\wp-\frac{2}{3} A^{2} \wp^{\prime \prime}+\frac{2}{3} A^{2} g_{2}\right) d z \\
\int_{\alpha} \phi_{1}(z) & =\int_{\alpha} \wp(z) d z-\frac{2}{3} A^{2} \int_{\alpha} \wp^{\prime \prime}(z) d z+\frac{2}{3} A^{2} g_{2} \int_{\alpha} d z \\
& =[-\zeta(z)]_{\alpha(0)}^{\alpha(1)}-\frac{2}{3} A^{2}\left[\wp^{\prime}(z)\right]_{\alpha(0)}^{\alpha(1)}+\frac{2}{3} A^{2} g_{2}[z]_{\alpha(0)}^{\alpha(1)} \\
& =-[\zeta(z)]_{\frac{i}{2}}^{\frac{i}{2}+1}-\frac{2}{3} A^{2}\left[\wp^{\prime}(z)\right]_{\frac{i}{2}}^{\frac{i}{2}+1}+\frac{2}{3} A^{2} g_{2}[z]_{\frac{i}{2}}^{\frac{i}{2}+1}
\end{aligned}
$$

Então,

$$
\int_{\alpha} \phi_{1}=-\left(\zeta\left(\frac{i}{2}+1\right)-\zeta\left(\frac{i}{2}\right)\right)-\frac{2}{3} A^{2}\left(\wp^{\prime}\left(\frac{i}{2}+1\right)-\wp^{\prime}\left(\frac{i}{2}\right)\right)+\frac{2}{3} A^{2} g_{2}\left(\frac{i}{2}+1-\frac{i}{2}\right) \text {. }
$$

Como, $\wp^{\prime}(i / 2+1)=\wp^{\prime}(i / 2)=0$, então:

$$
\int_{\alpha} \phi_{1}(z)=\zeta\left(\frac{i}{2}\right)-\zeta\left(\frac{i}{2}+1\right)+\frac{2}{3} A^{2} g_{2}
$$

Usando a fórmula:

$$
\begin{gathered}
\zeta\left(z+w_{1}\right)=\zeta(z)+2 \zeta\left(\frac{w_{1}}{2}\right), \text { para } z=\frac{i}{2} \text { e } w_{1}=1, \\
\text { segue, } \quad \zeta\left(\frac{i}{2}\right)-\zeta\left(\frac{i}{2}+1\right)=-2 \zeta\left(\frac{1}{2}\right) .
\end{gathered}
$$

Agora usando o ítem (iii) do lema 2.1.2, temos

$$
\zeta\left(\frac{i}{2}\right)-\zeta\left(\frac{i}{2}+1\right)=-2 \pi / 2=-\pi .
$$

Logo,

$$
\int_{\alpha} \phi_{1}(z)=-\pi+\frac{2}{3} A^{2} g_{2}
$$

A parte real do primeiro período se anula se, e só se,

$$
\operatorname{Re}\left\{\int_{\alpha} \phi_{1}\right\}=0 \Leftrightarrow-\pi+\frac{2}{3}\left(a^{2}-b^{2}\right) g_{2}=0, \quad \text { onde } A=a+i b, a, b \in \mathbb{R} .
$$

Logo,

porque, $g_{2}>0$

$$
a^{2}-b^{2}=\frac{3 \pi}{2 g_{2}}>0
$$


Cálculo do segundo período de $\phi_{1}$ :

$$
\begin{gathered}
\int_{\beta} \phi_{1}(z)=-[\zeta(z)]_{\beta(0)}^{\beta(1)}-\frac{2}{3} A^{2}\left[\wp^{\prime}(z)\right]_{\beta(0)}^{\beta(1)}+\frac{2}{3} A^{2} g_{2}[z]_{\beta(0)}^{\beta(1)} \\
=-[\zeta(z)]_{\frac{1}{2}}^{\frac{1}{2}+i}-\frac{2}{3} A^{2}\left[\wp^{\prime}(z)\right]_{\frac{1}{2}}^{\frac{1}{2}+i}+\frac{2}{3} A^{2} g_{2}[z]_{\frac{1}{2}}^{\frac{1}{2}+i} \\
\int_{\beta} \phi_{1}=-\zeta\left(\frac{1}{2}+i\right)+\zeta\left(\frac{1}{2}\right)-\frac{2}{3} A^{2}\left(\wp^{\prime}\left(\frac{1}{2}+i\right)-\wp^{\prime}\left(\frac{1}{2}\right)\right)+\frac{2}{3} A^{2} g_{2}\left(\frac{1}{2}+i-\frac{1}{2}\right) \\
=-\zeta\left(\frac{1}{2}+i\right)+\zeta\left(\frac{1}{2}\right)-\frac{2}{3} A^{2}\left(\wp^{\prime}\left(\frac{1}{2}\right)-\wp^{\prime}\left(\frac{1}{2}\right)\right)+\frac{2}{3} A^{2} g_{2} . i \\
=-\zeta\left(\frac{1}{2}+i\right)+\zeta\left(\frac{1}{2}\right)+\frac{2}{3} A^{2} g_{2} \cdot i
\end{gathered}
$$

Usando a fórmula $\zeta\left(z+w_{2}\right)=\zeta(z)+2 \zeta\left(\frac{w_{2}}{2}\right)$, e fazendo $z=\frac{1}{2}$ e $w_{2}=i$, temos

$$
\zeta\left(\frac{1}{2}+i\right)=\zeta\left(\frac{1}{2}\right)+2 . \zeta\left(\frac{i}{2}\right) \Rightarrow \zeta\left(\frac{1}{2}\right)-\zeta\left(\frac{1}{2}+i\right)=-2 . \zeta\left(\frac{i}{2}\right) .
$$

Pelo ítem (ii) e (iii) do lema 2, segue-se $\zeta\left(\frac{1}{2}\right)-\zeta\left(\frac{1}{2}+i\right)=2 i \cdot \frac{\pi}{2}=\pi \cdot i$ Portanto,

$$
\begin{gathered}
\int_{\beta} \phi_{1}(z)=\pi \cdot i+\frac{2}{3} i\left(a^{2}-b^{2}+2 a b i\right) g_{2}=\left(\pi+\frac{2}{3}\left(a^{2}-b^{2}\right) g_{2}\right) i-\frac{4}{3} a b g_{2} \\
\operatorname{Re}\left\{\int_{\beta} \phi_{1}\right\} \Leftrightarrow-\frac{4}{3} a \cdot b \cdot g_{2}=0 \Leftrightarrow a \cdot b=0
\end{gathered}
$$

Se $a=0$, então da equação (2.4) temos $-b^{2}=3 \pi / 2 g_{2}>0$, absurdo.

Logo $b=0$, entào $A=a$ e $A= \pm \sqrt{3 \pi / 2 g_{2}}$.

Similarmente podemos calcular os períodos de $\phi_{2}$ :

$$
\begin{aligned}
\int_{\alpha} \phi_{2} & =\int_{\alpha}\left(i \wp+\frac{2}{3} i A^{2} \wp^{\prime \prime}-\frac{2}{3} i A^{2} g_{2}\right) d z \\
& =-i[\zeta(z)]_{\frac{i}{2}}^{\frac{1}{2}+1}+\frac{2}{3} i A^{2}\left[\wp^{\prime}\right]_{\frac{i}{2}}^{\frac{i}{2}+1}-\frac{2}{3} i A^{2} g_{2}[z]_{\frac{i}{2}}^{\frac{i}{2}+1} \\
& =-i\left(\zeta\left(\frac{i}{2}+1\right)-\zeta\left(\frac{i}{2}\right)\right)-\frac{2}{3} i A^{2} g_{2}
\end{aligned}
$$




$$
\begin{aligned}
\int_{\alpha} \phi_{2} & =i\left(\zeta\left(\frac{i}{2}\right)-\zeta\left(\frac{i}{2}+1\right)\right)-\frac{2}{3} i A^{2} g_{2}=-2 i \zeta\left(\frac{1}{2}\right)-\frac{2}{3} i A^{2} g_{2} \\
& =-2 i \frac{\pi}{2}-\frac{2}{3} i A^{2} g_{2}=-\pi i-\frac{2}{3} i \cdot \frac{3 \pi}{2 g_{2}} \cdot g_{2}=-2 \pi i \\
\int_{\beta} \phi_{2} & =-i\left(\zeta\left(\frac{1}{2}+i\right)-\zeta\left(\frac{1}{2}\right)\right)-\frac{2}{3} i A^{2} g_{2} i \\
& =i\left(\zeta\left(\frac{1}{2}\right)-\zeta\left(\frac{1}{2}+i\right)\right)+\pi=i(\pi i)+\pi=0
\end{aligned}
$$

Portanto,

$$
\operatorname{Re}\left\{\int_{\alpha} \phi_{2}\right\}=\operatorname{Re}\left\{\int_{\beta} \phi_{2}\right\}=0
$$

Por último, calculamos os períodos de $\phi_{3}$ :

$$
\begin{gathered}
\int_{\alpha} \phi_{3}=\int_{\alpha} 2 A \wp^{\prime}(z) d z=2 A \int_{\alpha} \wp^{\prime}(z) d z=\left.2 A \wp(z)\right|_{\alpha(0)} ^{\alpha(1)}=2 A[\wp(z)]_{\frac{i}{2}}^{\frac{i}{2}+1} \\
=2 A\left[\wp\left(\frac{i}{2}+1\right)-\wp\left(\frac{i}{2}\right)\right]=2 A\left(\wp\left(\frac{i}{2}\right)-\wp\left(\frac{i}{2}\right)\right)=0 \\
\int_{\beta} \phi_{3}=2 A[\wp(z)]_{\frac{1}{2}}^{\frac{1}{2}+i}=2 A\left(\wp\left(\frac{1}{2}+i\right)-\wp\left(\frac{1}{2}\right)\right)=2 A\left(\wp\left(\frac{1}{2}\right)-\wp\left(\frac{1}{2}\right)\right)=0
\end{gathered}
$$

Usei aqui o fato de $\wp$ ser elíptica com períodos primitivos $w_{1}=1$ e $w_{1}=i$.

Portanto,

$$
\operatorname{Re}\left\{\int_{\alpha} \phi_{3} d z\right\}=\operatorname{Re}\left\{\int_{\beta} \phi_{3} d z\right\}=0
$$

$\mathrm{O}$ que prova $\mathrm{c}_{2}$.

\section{Demonstração de $c_{3}$ :}

Para demonstrar $c_{3}$, precisamos mostrar que o resíduo de $\phi_{j}$ em $P_{1}=0$ é real. Em símbolos $\operatorname{Res}\left(\phi_{j} ; 0\right) \in \mathbb{R} \forall j=1,2,3$, ou equivalentemente:

$$
\operatorname{Res}(g \eta ; 0)=\operatorname{Res}(g f d z ; 0)=\operatorname{Res}\left(\phi_{3} ; 0\right) \in \mathbb{R} \text { e } \overline{\operatorname{Res}(f d z ; 0)}+\operatorname{Res}\left(g^{2} f d z ; 0\right)=0 .
$$


Sendo $\eta=f d z=2 \wp(z) d z$, temos

$$
\operatorname{Res}(g f d z ; 0)=\operatorname{Res}\left(2 A \wp^{\prime}(z) d z ; 0\right)=2 A \operatorname{Res}\left(\wp^{\prime}(z) d z ; 0\right) .
$$

Mas, $\wp^{\prime}(z)$ é uma função elíptica, logo a soma de seus resíduos em $\bar{R}_{(1, i)}$ é igual a zero. Como o único polo de $f d z, g f d z$ e $g^{2} f d z$ em $\bar{R}(1, i)$ é o zero, temos

$$
\operatorname{Res}(g f d z ; 0)=0 \in \mathbb{R} .
$$

Pelo mesmo argumento, segue

$$
\operatorname{Res}(f d z ; 0)=2 \operatorname{Res}(\wp(z) d z ; 0)=0 \Rightarrow \overline{\operatorname{Res}(f d z ; 0)}=\overline{0}=0 .
$$

Por último, usando o ítem (iv) do lema 2.1.2

$$
g^{2} f d z=2 \wp(z) \frac{A^{2} \wp^{\prime 2}(z)}{\wp^{2}(z)} d z=2 A^{2} \frac{\wp^{\prime 2}(z)}{\wp(z)} d z=8.4^{2} \frac{\wp(z)}{\wp(z)}\left(\wp^{2}(z)-e_{1}^{2}\right) d z
$$

$g^{2} f d z=8 A^{2}\left(\varsigma^{2}(z)-e_{1}{ }^{2}\right) d z$. Logo, $g^{2} f$ é uma função elíptica e, $\operatorname{Res}\left(g^{2} f d z ; 0\right)=0$, pelo argumento acima. Portanto, temos $\mathbf{c}_{3}$.

\section{Demonstração de $c_{4}$ :}

Queremos mostrar que toda curva $\gamma:[0,1) \longrightarrow \mathbb{C}$ divergente em $R_{(1, i)}$, possui comprimento infinito. Feito isso a imersão $X: R_{(1, i)} \longrightarrow \mathbb{R}^{3}$ que defini $S$ é completa. Seja $d s=\frac{1}{2}|f|\left(1+|g|^{2}\right)|d z|=\lambda|d z|$ a métrica induzida por $X$. Vamos mostrar que

$$
\operatorname{comp}(\gamma)=\int_{\gamma} d s=\int_{\gamma} \frac{1}{2}|f|\left(1+|g|^{2}\right)|d z|=\int_{\gamma} \lambda|d z|=\infty .
$$

A fim de que $\gamma$ seja divergente em $R_{(1, i)}$, então $\lim _{t \rightarrow 1^{-}} \gamma(t)=0$. Agora como $f$ tem um polo de ordem 2 em $z=0$ e $g$ tem um polo simples em $z=0$, então $f\left(1+g^{2}\right)$ tem um polo de ordem $4 \mathrm{em} z=0$. Portanto na vizinhança $B_{\epsilon}(0)$ de zero, esta função pode ser escrita como, $\frac{1}{z^{4}} h(z)$, onde $h(z)$ é holomorfa em $B_{\epsilon}(0)$ e $h(0) \neq 0$. Mas, os zeros de uma função holomorfa são isolados, então existe $\epsilon_{1}<\epsilon$, de modo que $h(z) \neq 0, \quad \forall z \in B_{\epsilon_{1}}(0)$. É possível encontrar uma constante $M>0$ tal que $|h(z)| \geq M, \quad \forall z \in B_{\epsilon_{1}}(0)$. Consideremos $\left.\gamma\right|_{\left[t_{i}, 1\right)} \subset B_{\epsilon_{1}}(0), \quad t_{i}>0$, em outras palavras é a parte de $\gamma$ que cai dentro de $B_{\varepsilon_{1}}(0)$ 


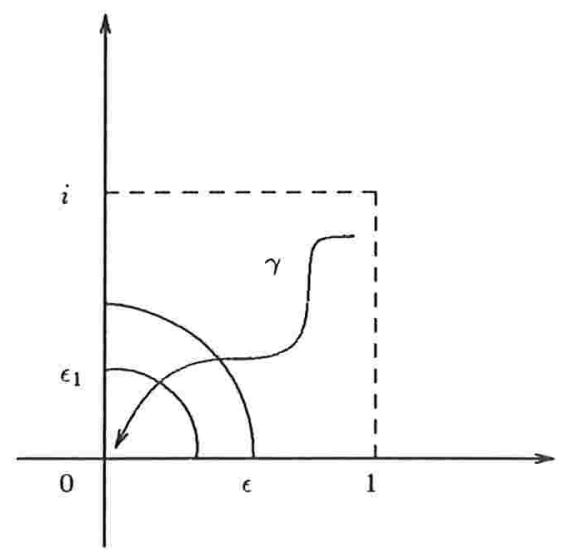

\section{Figura 2.3}

Obviamente,

$$
\begin{aligned}
& \operatorname{comp}(\gamma)>\operatorname{comp}\left(\left.\gamma\right|_{\left[t_{t}, 1\right)}\right)=\int_{\left.\gamma\right|_{\left(t_{i}, 1\right)}} \frac{1}{2}|f|\left(1+|g|^{2}\right)|d z| \geq \int_{\gamma \mid{ }_{\left(t_{i}, 1\right)}}\left|\frac{1}{2} f\left(1+g^{2}\right)\right||d z| \\
& \operatorname{comp}(\gamma) \geq \int_{\left.\gamma\right|_{\left(t_{i}, 1\right)}} \frac{1}{2|z|^{4}} \cdot|h(z)||d z| \\
& \operatorname{comp}(\gamma) \geq M \int_{\left.\gamma\right|_{\left(t_{i}, 1\right)}} \frac{1}{2|z|^{4}}|d z| \geq \frac{M}{2}\left|\int_{\gamma_{\left(t_{i}, 1\right)}} \frac{d z}{z^{4}}\right| .
\end{aligned}
$$

Seja $t_{0} \in\left[t_{i}, 1\right)$ o primeiro valor de $t$, tal que $\gamma\left(t_{0}\right) \in B_{\epsilon_{1}}$, escrevemos $\gamma\left(t_{0}\right)=\zeta_{0}$ e $t_{i} \rightarrow 1^{-}, \gamma\left(t_{i}\right)=\zeta$

$$
\begin{aligned}
& \operatorname{comp}(\gamma) \geq \frac{M}{2}\left|\lim _{\zeta \rightarrow 0} \int_{\zeta_{0}}^{\zeta} \frac{1}{z^{4}} d z\right|=\frac{M}{2}\left|\lim _{\zeta \rightarrow 0}\left[-\frac{1}{3 z^{3}}\right]_{\zeta_{0}}^{\zeta}\right| \\
& \operatorname{comp}(\gamma) \geq \frac{M}{6}\left|\lim _{\zeta \rightarrow 0}\left(\frac{1}{\zeta_{0}^{3}}-\frac{1}{\zeta^{3}}\right)\right| \rightarrow \infty
\end{aligned}
$$


Em [21] K. Sato esboça o gráfico da superfície descrita no teorema acima, e usando o software mathematica 3.0 ver [10] construímos esse gráfico.

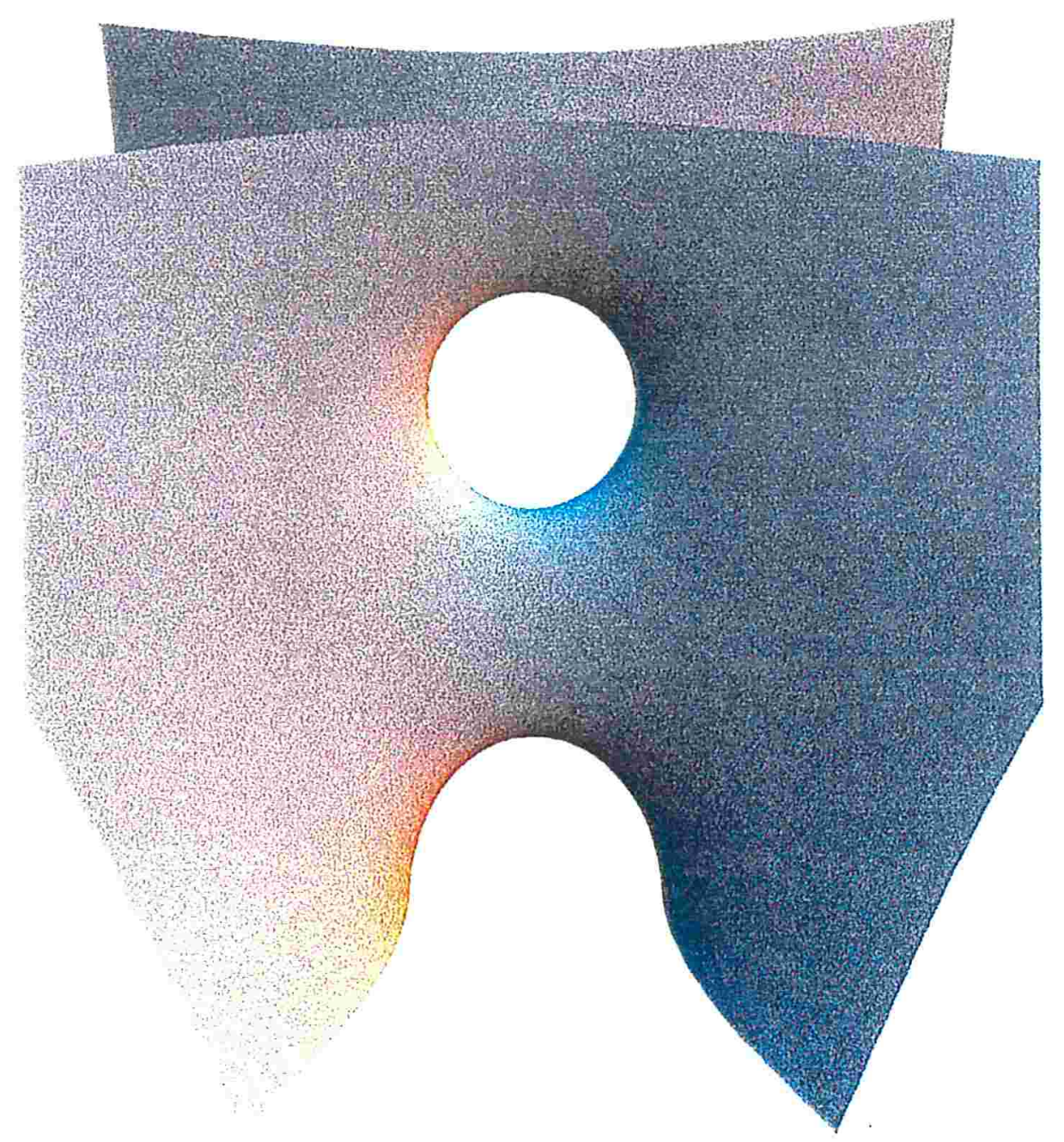




\section{Capítulo 3}

\section{Superfície Mínima de \\ Chen-Gackstätter de Gênero 2}

Neste capítulo faremos inicialmente uma adaptação à linguagem hiperelíptica da representação de Weierstrass exibida no capítulo 2. E usando a teoria de superfícies de Riemann de funções algébricas, demonstraremos que existe uma superfície mínima completa de gênero 2 e curvatura total finita $-12 \pi$ do $\mathbb{R}^{3}$

\subsection{Adaptação à Linguagem Hiperelíptica do Exem- plo de gênero 1}

Para determinar uma superfície mínima com gênero $p>1$ é natural iniciar com uma superfície de Riemann hiperelíptica $\bar{R}$, a qual é realizada por uma função algébrica. Para ter uma idéia melhor do que faremos é interessante adaptar o teorema 2.2 .1 à essa linguagem, veja figura 3.1 e as considerações a seguir. 


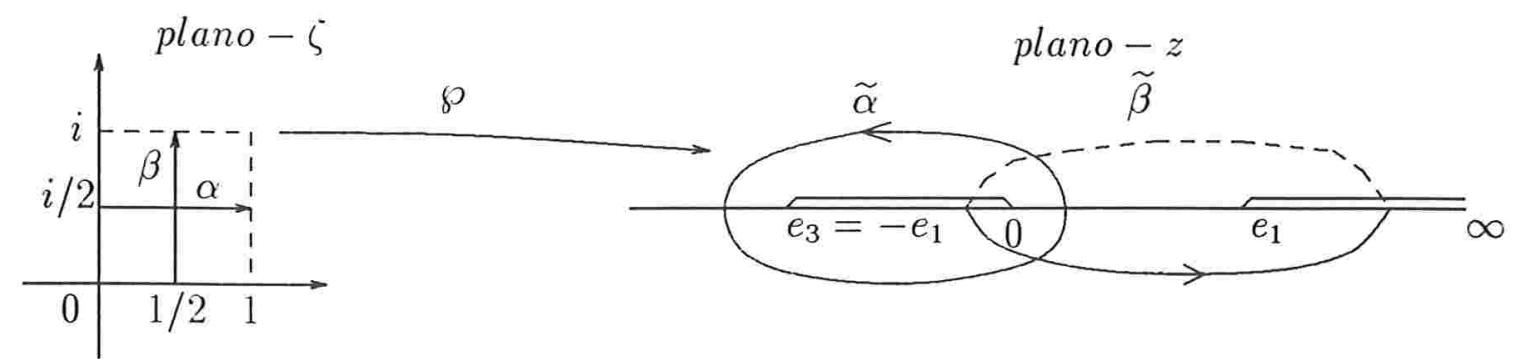

Figura 3.1

onde a parte pontilhada de $\widetilde{\beta}$, significa que pertence a folha II da superfície de Riemann compacta $\bar{R}$, a qual é realizada pela função algébrica

$$
w(z):=2 \sqrt{z\left(z-e_{1}\right)\left(z+e_{2}\right)} .
$$

Da equação diferencial de $\wp$ dada em 1.15 , temos que $\wp^{\prime}(\zeta)$, satisfaz a equação algébrica,

$$
\left.[w(\wp(\zeta))]^{2}=4 \wp(\zeta)\left(\wp(\zeta)-e_{1}\right)\left(\wp(\zeta)+e_{1}\right)\right)
$$

Pondo, $z=\rho(\zeta), d z=\rho^{\prime}(\zeta) d \zeta$, de 3.1 , segue

$$
w^{2}(z)=4 z\left(z-e_{1}\right)\left(z+e_{1}\right) .
$$

Logo, $d \zeta=\frac{1}{\rho^{\prime}(\zeta)} d z=\frac{d z}{w(z)}$, e portanto, temos $g(\zeta)$ e $\eta(\zeta)=f(\zeta) d \zeta$ como função de $w(z)$.

$$
\begin{gathered}
g(\zeta)=A \cdot \frac{\wp^{\prime}(\zeta)}{\wp(\zeta)} \quad \text { corresponde a } \quad \tilde{g}(z)=A \cdot \frac{2 \sqrt{z\left(z^{2}-e_{1}^{2}\right)}}{z} \\
\eta(\zeta)=2 \wp(\zeta) d \zeta \quad \text { corresponde a } \quad \tilde{\eta}(z)=\frac{z \cdot d z}{\sqrt{z\left(z^{2}-e_{1}^{2}\right)}}
\end{gathered}
$$

onde $\widetilde{\eta}(z)$ é uma diferencial abeliana sobre $\bar{R} ; \bar{R}$ é homeomorfo ao toro, e $\{\tilde{\alpha}, \tilde{\beta}\}$ forma uma base para sua homologia, veja figura 3.2 abaixo. 

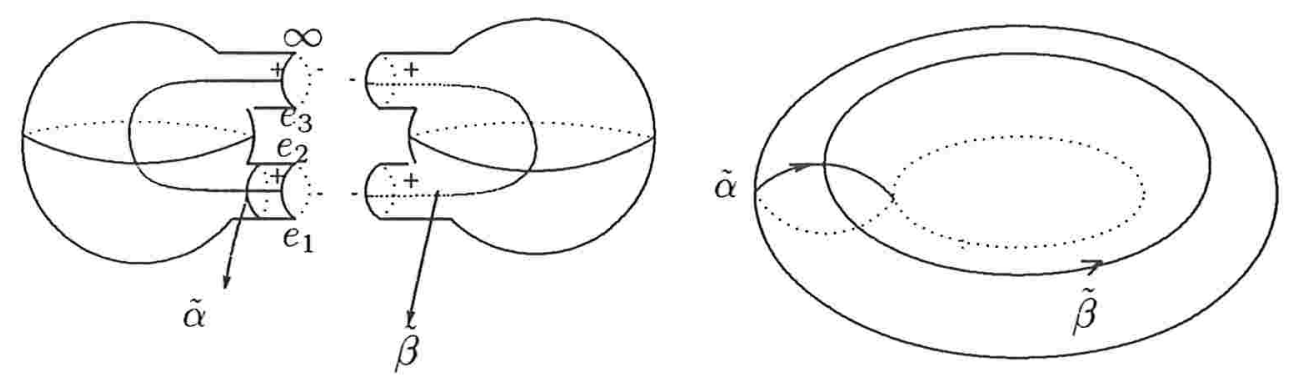

Figura 3.2

\subsection{Exemplo de Chen-Gackstätter de gênero 2}

O exemplo de Chen-Gackstätter de uma superfície mínima completa em $\mathbb{R}^{3}$, de gênero 2 , consiste na imersão em $\mathbb{R}^{3}$ de uma superfície de Riemann compacta, da funçào algébrica: $w(z)=\sqrt{z\left(z^{2}-a^{2}\right)\left(z^{2}-b^{2}\right)}$, onde $a, b \in \mathbb{R}, \quad 0<a<b$, menos um ponto. Mais precisamente, temos:

Teorema 3.2.1 Existe uma superfície mínima completa $S$ do $\mathbb{R}^{3}$ com gênero 2 , curvatura total finita $C(S)=-12 \pi$ e um único fim de ordem 3. Esta superfície é do tipo Enneper com duas alças. Ela é a imersão da superfície de Riemann $\bar{R}$, da função algébrica,

$$
w(z):=\sqrt{z\left(z^{2}-a^{2}\right)\left(z^{2}-b^{2}\right)}
$$

menos um ponto de ramificação correspondente ao infinito. A representação de Weierstrass de $S$ é dada por:

$$
\left\{\begin{array}{l}
g(z)=B \frac{w(z)}{\left(z^{2}-a^{2}\right)} \\
\eta(z)=\frac{\left(z^{2}-a^{2}\right)}{w(z)} d z
\end{array}\right.
$$

onde $a, b$ e $B$ são constantes reais convenientes satisfazendo, $0<a<b$ e $0<B$. 
Antes de começar a demonstração do teorema acima, precisamos de alguns resultados obtidos nos lemas a seguir.

Seja $\bar{R}$ a superfície de Riemann da função algébrica:

$$
w(z)=\left[z\left(z^{2}-a^{2}\right)^{k}\left(z^{2}-b^{2}\right)\right]^{\frac{1}{k+1}}, \quad \text { onde } a, b \in \mathbb{R}, 0<a<b, \text { e } k \in \mathbb{Z}_{+}
$$

Temos o lema:

Lema 3.2.2 Sejam $\phi_{1}(z)$ e $\phi_{2}(z)$ diferenciais meromorfas sobre uma superfície de Riemann compacta $\bar{R}$, definidas por:

$$
\begin{aligned}
& \phi_{1}(z):=\frac{1}{2}\left\{\frac{\left[z\left(z^{2}-a^{2}\right)^{k}\left(z^{2}-b^{2}\right)\right]^{\frac{1}{k+1}}}{z\left(z^{2}-b^{2}\right)}-B^{2} \frac{z\left(z^{2}-b^{2}\right)}{\left[z\left(z^{2}-a^{2}\right)^{k}\left(z^{2}-b^{2}\right)\right]^{\frac{1}{k+1}}}\right\} d z \\
& \phi_{2}(z):=\frac{i}{2}\left\{\frac{\left[z\left(z^{2}-a^{2}\right)^{k}\left(z^{2}-b^{2}\right)\right]^{\frac{1}{k+1}}}{z\left(z^{2}-b^{2}\right)}+B^{2} \frac{z\left(z^{2}-b^{2}\right)}{\left[z\left(z^{2}-a^{2}\right)^{k}\left(z^{2}-b^{2}\right)\right]^{\frac{1}{k+1}}}\right\} d z
\end{aligned}
$$

onde $a, b$ e $B$ são constantes reais convenientes satisfazendo, $0<a<b$ e $0<B$. Então valem as seguintes igualdades:

(i) $\int_{c_{1}} \phi_{1}(z)=\int_{c_{1}} \phi_{2}(z)=0, \forall z \in c_{1}: z=\varepsilon e^{i t}, t \in[0,2 \pi]$, quando $\varepsilon \rightarrow 0$

(ii) $\int_{c_{2}} \phi_{1}(z)=\int_{c_{2}} \phi_{2}(z)=0, \forall z \in c_{2}: z=a+\varepsilon e^{i t}, t \in[-\pi, \pi]$, quando $\varepsilon \rightarrow 0$

(iii) $\int_{c_{3}} \phi_{1}(z)=\int_{c_{3}} \phi_{2}(z)=0, \forall z \in c_{3}: z=-b+\varepsilon e^{i t}, t \in[0,2 \pi]$, quando $\varepsilon \rightarrow 0$

(iv) $\int_{c_{4}} \phi_{1}(z)=\int_{c_{4}} \phi_{2}(z)=0, \forall z \in c_{4}: z=-a+\varepsilon e^{i t}, t \in[-\pi, \pi]$, quando $\varepsilon \rightarrow 0$

(v) $\int_{\Gamma_{1}} \phi_{1}(z)=\int_{\Gamma_{1}} \phi_{2}(z)=0, \forall z \in \Gamma_{1}: z=a+\varepsilon e^{i t}, t \in[-\pi, 3 \pi]$, quando $\varepsilon \rightarrow 0$

(vi) $\int_{\Gamma_{2}} \phi_{1}(z)=\int_{\Gamma_{2}} \phi_{2}(z)=0, \forall z \in \Gamma_{2}: z=b+\varepsilon e^{i t}, t \in[0,4 \pi]$, quando $\varepsilon \rightarrow 0$

(vii) $\int_{\Gamma_{3}} \phi_{1}(z)=\int_{\Gamma_{3}} \phi_{2}(z)=0, \forall z \in \Gamma_{3}: z=-a+\varepsilon e^{i t}, t \in[-\pi, 3 \pi]$, quando $\varepsilon \rightarrow 0$ 
(viii) $\int_{\Gamma_{4}} \phi_{1}(z)=\int_{\Gamma_{4}} \dot{o}_{2}(z)=0, \forall z \in \Gamma_{4}: z=\varepsilon e^{i t}, t \in[0,4 \pi]$, quando $\varepsilon \rightarrow 0$

Onde as curvas $c_{1}, c_{2}, c_{3}$ e $c_{4}$ pertencem somente a uma das $j$ folhas

$\mathbb{C} \backslash\{[-b,-a] \cup[0, a] \cup[b, \infty)\}, j=1,2, \ldots, k+1$, de $w(z)=\left[z\left(z^{2}-a^{2}\right)^{k}\left(z^{2}-b^{2}\right)\right]^{\frac{1}{k+1}}$, com $k$ inteiro positivo. usadas na construção de $\bar{R}$, e o argumento de cada ramo de $w(z)$ na parte superior dos cortes das folhas $j$, é $\frac{2 j \pi}{k+1}$. Já as curvas $\Gamma_{1}, \Gamma_{2}, \Gamma_{3}$ e $\Gamma_{4}$ passam por duas das $k+1$ folhas de $w(z)$.

\section{Demonstração:}

Provaremos que $\left|\int \phi_{1}(z)\right| \rightarrow 0$ e $\left|\int \phi_{2}(z)\right| \rightarrow 0$, para todo $z$ nas condições dos oito ítens acima. De um modo geral, para cada ramo de $w(z)$, valem as desigualdades:

$$
\begin{aligned}
\left|\int \phi_{1}(z)\right| & \leq \frac{1}{2} \int\left|\frac{e^{\frac{2 \jmath \pi i}{k+1}}\left[z\left(z^{2}-a^{2}\right)^{k}\left(z^{2}-b^{2}\right)\right]^{\frac{1}{k+1}}}{z\left(z^{2}-b^{2}\right)}\right||d z|+ \\
& +\frac{B^{2}}{2} \int\left|\frac{z\left(z^{2}-b^{2}\right)}{e^{\frac{2 \pi \pi i}{k+1}}\left[z\left(z^{2}-a^{2}\right)^{k}\left(z^{2}-b^{2}\right)\right]^{\frac{1}{k+1}}}\right||d z| \leq \\
& \leq \frac{1}{2} \int\left[\frac{\left|z^{2}-a^{2}\right|}{|z|\left(\left|z^{2}-b^{2}\right|\right)}\right]^{\frac{k}{k+1}}|d z|+\frac{B^{2}}{2} \int\left[\frac{|z|\left(\left|z^{2}-b^{2}\right|\right)}{\left(\left|z^{2}-a^{2}\right|\right)}\right]^{\frac{k}{k+1}}|d z|
\end{aligned}
$$

Como $0<a<b$,

$$
\left|\int \phi_{1}(z)\right| \leq \frac{1}{2} \int\left[\frac{|z+a||z-a|}{|z|\left(\left.\left|a^{2}-\right| z\right|^{2} \mid\right)}\right]^{\frac{k}{k+1}}|d z|+\frac{B^{2}}{2} \int\left[\frac{|z|\left(|z|^{2}+b^{2}\right)}{\left(\left.\left|a^{2}-\right| z\right|^{2} \mid\right)}\right]^{\frac{k}{k+1}}|d z|
$$

(i) Seja $\varepsilon \leq a / 2$ e $c_{1}=\varepsilon e^{i t}, t \in[0,2 \pi]$, como na figura 3.3 abaixo,

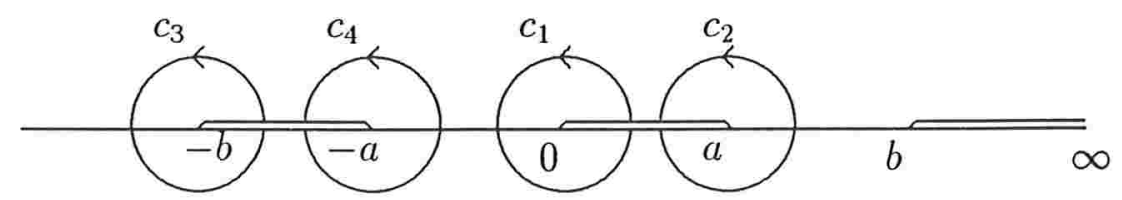

Figura 3.3 
Logo,

$$
\begin{aligned}
& \left|\int_{c_{1}} \phi_{1}(z)\right| \leq \frac{1}{2} \int_{c_{1}}\left[\frac{|z+a||z-a|}{|z|\left(a^{2}-|z|^{2}\right)}\right]^{\frac{k}{k+1}}|d z|+\frac{B^{2}}{2} \int_{c_{1}}\left[\frac{|z|\left(|z|^{2}+b^{2}\right)}{\left(a^{2}-|z|^{2}\right)}\right]^{\frac{k}{k+1}}|d z| \\
& \left|\int_{c_{1}} \phi_{1}(z)\right| \leq \frac{1}{2} \int_{c_{1}}\left[\frac{|z|+a}{|z|(a-|z|)}\right]^{\frac{k}{k+1}}|d z|+\frac{B^{2}}{2} \int_{c_{1}}\left[\frac{|z|\left(|z|^{2}+b^{2}\right)}{\left(a^{2}-|z|^{2}\right)}\right]^{\frac{k}{k+1}}|d z|
\end{aligned}
$$

Substituindo acima $|z|=\varepsilon$ e $|d z|=\varepsilon d t$, temos

$$
\begin{aligned}
& \left|\int_{c_{1}} \phi_{1}(z)\right| \leq \frac{1}{2}\left[\frac{\varepsilon+a}{\varepsilon(a-\varepsilon)}\right]^{\frac{k}{k+1}} \int_{0}^{2 \pi} \varepsilon d t+\frac{B^{2}}{2}\left[\frac{\varepsilon\left(\varepsilon^{2}+b^{2}\right)}{\left(a^{2}-\varepsilon^{2}\right)}\right]^{\frac{k}{k+1}} \int_{0}^{2 \pi} \varepsilon d t \quad \text { Assim, } \\
& \left|\int_{c_{1}} \phi_{1}(z)\right| \leq\left\{\left[\frac{\varepsilon+a}{(a-\varepsilon)}\right]^{\frac{k}{k+1}} \varepsilon^{\frac{1}{k+1}}+B^{2}\left[\frac{\left(\varepsilon^{2}+b^{2}\right)}{\left(a^{2}-\varepsilon^{2}\right)}\right]^{\frac{k}{k+1}} \varepsilon^{\frac{2 k+1}{k+1}}\right\} \pi
\end{aligned}
$$

Portanto, fazendo $\varepsilon \rightarrow 0$, implica $\left|\int \phi_{1}(z)\right| \rightarrow 0$. Também $\left|\int \phi_{2}(z)\right| \rightarrow 0$. para todo $z \in c_{1}$ e $\varepsilon \rightarrow 0$, já que vale novamente a desigualdade 3.3 , com $\phi_{2}$ no lugar de $\phi_{1}$. (ii) Sejam $\varepsilon \leq \frac{b-a}{2},|z-a|=\varepsilon$ e $c_{2}: z=a+\varepsilon e^{i t}, t \in[-\pi, \pi]$. Além disso, valem as desigulades:

$$
\begin{aligned}
& |z+a|=|z-a+2 a| \geq 2 a-|z-a| \quad \text { e }|z+a| \leq 2 a+|z-a| \\
& |z+b|=|z-a+(b+a)| \geq(b+a)-|z-a| \text { e }|z+b| \leq(b+a)+|z-a| \\
& |z-b|=|z-a-(b-a)| \geq(b-a)-|z-a| \text { e }|z-b| \leq(b-a)+|z-a| \\
& |z|=|z-a+a| \geq a-|z-a| \text { e }|z| \leq a+|z-a|
\end{aligned}
$$

Usando-as na inequação 3.3 temos,

$$
\begin{aligned}
\left|\int_{c_{2}} \phi_{1}(z)\right| \leq & \frac{1}{2} \int_{c_{2}}\left[\frac{|z-a|(2 a+|z-a|)}{(a-|z-a|)((b+a)-|z-a|)((b-a)-|z-a|)}\right]^{\frac{k}{k+1}}|d z|+ \\
& +\frac{B^{2}}{2} \int_{c_{2}}\left[\frac{(|z-a|+a)(|z-a|+(b+a))(|z-a|+(b-a))}{(2 a-|z-a|)|z-a|}\right]^{\frac{k}{k+1}}|d z|
\end{aligned}
$$




$$
\begin{aligned}
\left|\int_{c_{2}} \phi_{1}(z)\right| \leq & \frac{1}{2}\left[\frac{\varepsilon(2 a+\varepsilon)}{(a-\varepsilon)((b+a)-\varepsilon)((b-a)-\varepsilon)}\right]^{\frac{k}{k+1}} \varepsilon \int_{-\pi}^{\pi} d t+ \\
& +\frac{B^{2}}{2}\left[\frac{(\varepsilon+a)(\varepsilon+(b+a))(\varepsilon+(b-a))}{(2 a-\varepsilon) \varepsilon}\right]^{\frac{k}{k+1}} \varepsilon \int_{-\pi}^{\pi} d t \\
& \left|\int_{c_{2}} \phi_{1}(z)\right| \leq\left\{\left[\frac{(2 a+\varepsilon)}{(a-\varepsilon)((b+a)-\varepsilon)((b-a)-\varepsilon)}\right]^{\frac{k}{k+1}} \varepsilon^{\frac{2 k+1}{k+1}}+\right. \\
& \left.+B^{2}\left[\frac{(\varepsilon+a)(\varepsilon+(b+a))(\varepsilon+(b-a))}{(2 a-\varepsilon)}\right]^{\frac{k}{k+1}} \varepsilon^{\frac{1}{k+1}}\right\} \pi
\end{aligned}
$$

Agora fazendo, $\varepsilon \rightarrow 0$ na expressão acima resulta, $\left|\int_{c_{2}} \phi_{1}(z)\right| \rightarrow 0$, assim como $\left|\int_{c_{2}} \phi_{2}(z)\right| \rightarrow 0$, com $\varepsilon \rightarrow 0$, usando os mesmo tipo de cálculo. A demonstração dos ítens (iii) e (iv) é análoga a do ítem (ii).

(v) Seja $\Gamma_{1}=\Gamma_{1}^{j} \cup \Gamma_{1}^{j-1}$, onde $\Gamma_{1}^{j}$ (linha cheia) e $\Gamma_{1}^{j-1}$ (linha pontilhada) são as partes de $\Gamma_{1}$ que pertencem respectivamente às folhas $j$ e $j-1$, indicadas na figura 3.4 abaixo.

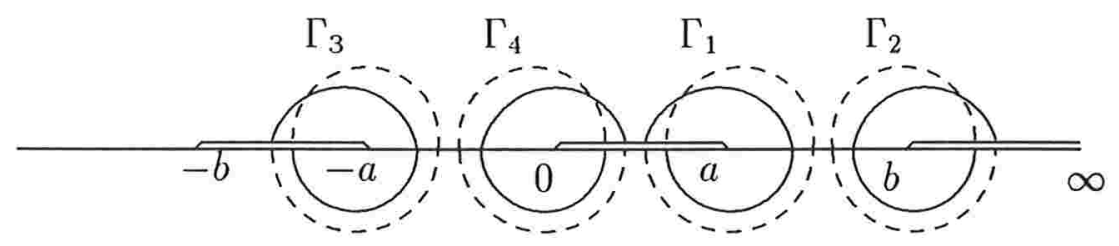

Figura 3.4

Então,

$$
\begin{gathered}
\int_{\Gamma_{1}} \phi_{1}(z)=\int_{\Gamma_{1}^{j}} \phi_{1}(z)+\int_{\Gamma_{1}^{j-1}} \phi_{1}(z) \mathrm{e} \\
\int_{\Gamma_{1}} \phi_{1}(z)=\frac{1}{2} \int_{\Gamma_{1}^{j}} \frac{e^{\frac{2 \jmath \pi i}{k+1}}\left[z\left(z^{2}-a^{2}\right)^{k}\left(z^{2}-b^{2}\right)\right]^{\frac{1}{k+1}}}{z\left(z^{2}-b^{2}\right)} d z-
\end{gathered}
$$




$$
\begin{aligned}
- & \frac{B^{2}}{2} \int_{\Gamma_{1}^{j}} \frac{z\left(z^{2}-b^{2}\right)}{e^{\frac{2 j \pi i}{k+1}}\left[z\left(z^{2}-a^{2}\right)^{k}\left(z^{2}-b^{2}\right)\right]^{\frac{1}{k+1}}} d z+ \\
+ & \frac{1}{2} \int_{\Gamma_{1}^{j-1}} \frac{e^{\frac{2(j-1) \pi i}{k+1}}\left[z\left(z^{2}-a^{2}\right)^{k}\left(z^{2}-b^{2}\right)\right]^{\frac{1}{k+1}}}{z\left(z^{2}-b^{2}\right)} d z- \\
- & \frac{B^{2}}{2} \int_{\Gamma_{1}^{j-1}} \frac{z\left(z^{2}-b^{2}\right)}{e^{\frac{2(j-1) \pi i}{k+1}}\left[z\left(z^{2}-a^{2}\right)^{k}\left(z^{2}-b^{2}\right)\right]^{\frac{1}{k+1}}} d z \\
\left|\int_{\Gamma_{1}} \phi_{1}(z)\right| & \leq \frac{1}{2} \int_{\Gamma_{1}^{j}}\left|\frac{\left[z\left(z^{2}-a^{2}\right)^{k}\left(z^{2}-b^{2}\right)\right]^{\frac{1}{k+1}}}{z\left(z^{2}-b^{2}\right)}\right||d z|+ \\
& +\frac{B^{2}}{2} \int_{\Gamma_{1}^{j}}\left|\frac{z\left(z^{2}-b^{2}\right)}{\left[z\left(z^{2}-a^{2}\right)^{k}\left(z^{2}-b^{2}\right)\right]^{\frac{1}{k+1}}}\right||d z|+ \\
& +\frac{1}{2} \int_{\Gamma_{1}^{j-1}}\left|\frac{\left[z\left(z^{2}-a^{2}\right)^{k}\left(z^{2}-b^{2}\right)\right]^{\frac{1}{k+1}}}{z\left(z^{2}-b^{2}\right)}\right||d z|+ \\
& +\frac{B^{2}}{2} \int_{\Gamma_{1}^{j-1}}\left|\frac{z\left(z^{2}-b^{2}\right)}{\left[z\left(z^{2}-a^{2}\right)^{k}\left(z^{2}-b^{2}\right)\right]^{\frac{1}{k+1}}}\right||d z|
\end{aligned}
$$

Pela inequação 3.3 e ítem (ii), usadas em 3.4 temos $\left|\int_{\Gamma_{1}} \phi_{1}(z)\right| \rightarrow 0$, quando $\varepsilon \rightarrow 0$. E com um cálculo semelhante se prova os ítens (vi), (vii) e (viii).

\section{Observações:}

(1) Note que quando $k=1$, então $\bar{R}$ é uma superfície de Riemann compacta de gênero 2 , hiperelíptica e $j=1,2$, isto é, temos duas folhas, $\mathbb{C} \backslash\{[-b,-a] \cup[0, a] \cup[b, \infty)\}$, da função algébrica $w(z)=\sqrt{z\left(z^{2}-a^{2}\right)\left(z^{2}-b^{2}\right)}$.

(2) Ainda quando $k=1$, as expressões de $\phi_{1}$ e $\phi_{2}$ são dadas por:

$$
\begin{aligned}
& \phi_{1}(z)=\frac{1}{2}\left[\frac{\left(z^{2}-a^{2}\right)-B^{2} z\left(z^{2}-b^{2}\right)}{\sqrt{z\left(z^{2}-a^{2}\right)\left(z^{2}-b^{2}\right)}}\right] d z \\
& \phi_{2}(z)=\frac{i}{2}\left[\frac{\left(z^{2}-a^{2}\right)+B^{2} z\left(z^{2}-b^{2}\right)}{\sqrt{z\left(z^{2}-a^{2}\right)\left(z^{2}-b^{2}\right)}}\right] d z
\end{aligned}
$$


Lema 3.2.3 Seja $1<b<\infty$, então valem as seguintes desigualdades:

(i) $\quad \frac{1+x}{(b+x)(b-x)} \leq \frac{2}{b(b-1)}$, se $0 \leq x \leq 1$

(ii) $\quad \frac{(b-1+x)(b+1-x)}{(2-x)} \geq \frac{b(b-1)}{2}$, se $0 \leq x \leq 1$

(iii) $\quad \frac{(1+x)(b+1+x)}{2+x} \leq 2 b$, se $0 \leq x \leq b-1$

(iv) $\frac{b+1-x}{(b-x)(2 b-x)} \geq \frac{1}{2 b}$, se $0 \leq x \leq b-1$

\section{Demonstração:}

(i) Basta observar que se $0 \leq x \leq 1$, então $(1+x) \leq 2,(b+x) \geq b$ e $(b-x) \geq b-1$

(ii) Notemos que, $(2-x) \leq 2,(b-1+x) \geq(b-1)$ e $(b+1-x) \geq b$

(iii) Usando o mesmo tipo de argumento dos ítens anteriores, segue

$$
\frac{(1+x)(b+1+x)}{2+x} \leq \frac{(1+x)(b+1+x)}{1+x}=b+1+x \leq 2 b
$$

(iv) Segue de

$$
\frac{b+1-x}{(b-x)(2 b-x)} \geq \frac{b-x}{(b-x)(2 b-x)}=\frac{1}{(2 b-x)} \geq \frac{1}{2 b}
$$

Lema 3.2.4 Sejam $1<b<\infty$ e $\varepsilon \leq 0,21$, então valem as seguintes desigualdades:
(i) $\frac{1+x}{(1+\varepsilon+x)} \geq 0,81, \quad \forall 0 \leq x \leq 1 \quad$ e $\quad \forall 0<\varepsilon \leq \frac{19}{81} \approx 0,23$
(ii) $\frac{2+\varepsilon-x}{2-x} \leq 1,21, \quad \forall 0 \leq x \leq 1, \quad \forall 0<\varepsilon \leq 0,21$
(iii) $\quad \frac{(1+x)(2+\varepsilon+x)}{2+x} \geq 1$, se $0 \leq x \leq \varepsilon$
(iv) $\frac{2+\varepsilon-x}{(1+\varepsilon-x)(2+2 \varepsilon-x)} \leq 1, \quad$ se $0 \leq x \leq \varepsilon$
(v) $\quad \frac{0,9}{\sqrt{1+\varepsilon-x}} \geq(1,1) \sqrt{\varepsilon+x}, \quad \forall 0 \leq x \leq 1 \quad$ e $\quad \forall 0<\varepsilon \leq 0,21$ 


\section{Demonstração:}

(i) Vale a inequação $\frac{1+x}{(1+\varepsilon+x)} \geq 0,81 \Leftrightarrow \frac{(1+\varepsilon+x)}{1+x} \leq \frac{1}{0,81}$, mas $\frac{(1+\varepsilon+x)}{1+x}=\frac{1+x}{1+x}+\frac{\varepsilon}{1+x}=$ $1+\frac{\varepsilon}{1+x} \leq 1+\varepsilon$, pois $(1+x) \geq 1$. Logo, $(1+\varepsilon) \leq \frac{1}{0,81} \Leftrightarrow \varepsilon \leq \frac{19}{81} \approx 0,23$ implica $\frac{(1+\varepsilon+x)}{1+x} \leq \frac{1}{0,81}$.

(ii) Similarmente ao ítem anterior, podemos facilmente provar $\frac{2+\varepsilon-x}{(2-x)} \leq 1,21 \Leftrightarrow 0 \leq$ $\varepsilon \leq 0,21$.

(iii) É óbvio, basta observar que $1+x \geq 1,2+\varepsilon+x \geq 2+\varepsilon$ e $2+x \leq 2+\varepsilon$.

(iv) De fato, $\quad \frac{2+\varepsilon-x}{(1+\varepsilon-x)(2+2 \varepsilon-x)} \leq \frac{2+2 \varepsilon-x}{(1+\varepsilon-x)(2+2 \varepsilon-x)}=\frac{1}{(1+\varepsilon-x)} \leq 1$.

(v) Vale, $\frac{0,9}{\sqrt{1+\varepsilon-x}} \geq(1,1) \sqrt{\varepsilon+x} \Leftrightarrow 1,21(\varepsilon+x) \leq \frac{0,81}{1+\varepsilon-x} \Leftrightarrow(1+\varepsilon-x)(\varepsilon+x) \leq \frac{81}{121}$ como, $(1+\varepsilon-x)(\varepsilon+x)=(\varepsilon+x)+(\varepsilon+x)(\varepsilon-x)=(\varepsilon+x)+\varepsilon^{2}-x^{2}=\varepsilon^{2}+\varepsilon+\left(x-x^{2}\right) \Rightarrow$ $(1+\varepsilon-x)(\varepsilon+x) \leq \varepsilon^{2}+\varepsilon+\frac{1}{4}$, pois o máximo de $x-x^{2}, \quad \forall x \in[0,1]$ é $\frac{1}{4}$.

Logo reduzimos o problema em determinar valores de $\varepsilon$ que satisfaçam a inequação $\varepsilon^{2}+\varepsilon+\frac{1}{4} \leq \frac{81}{121}$. Esses valores para $\varepsilon$ estão no intervalo $(0,0,318]$. Portanto, $(v)$ vale $\forall \varepsilon \in(0,0,21]$ e $x \in[0,1]$
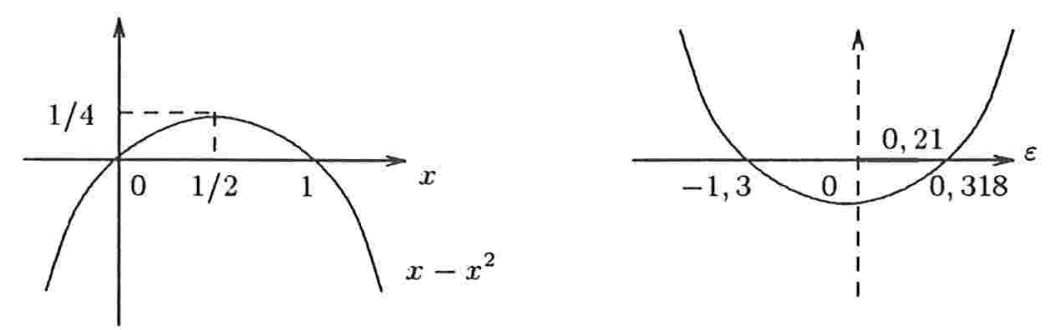

Figura 3.5

Abaixo começamos a demonstração do teorema 3.2.1

\section{Demonstração:}

Consideremos primeiramente as duas folhas usadas para construir a superfície de Riemann concreta $\bar{R}$ de $w(z)$, veja figura 3.6 . 

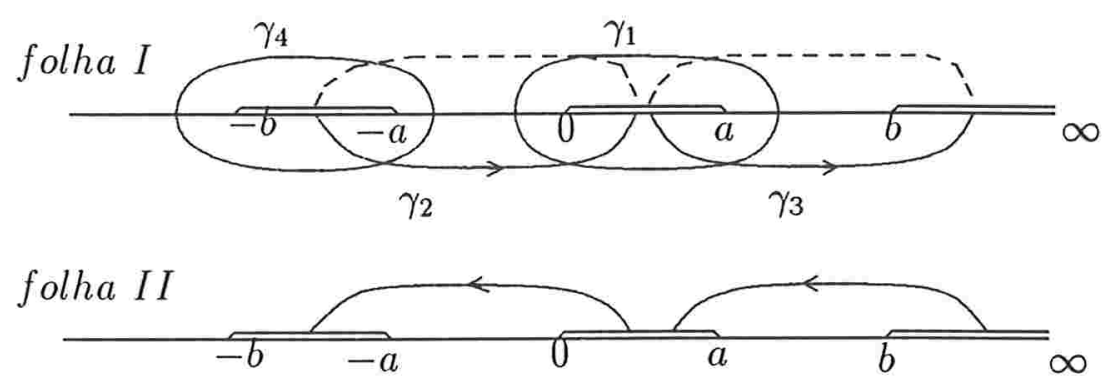

Figura 3.6

Homeomorficamente as figuras, 3.7, que se seguem, exprimem esquematicamente a situação acima.
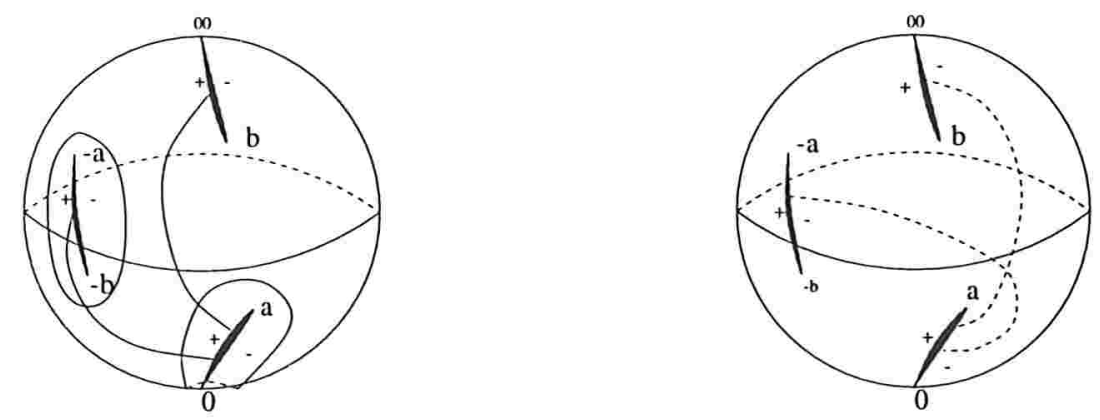

\section{Figura 3.7}

A superfície de Riemann de $w(z)$, é obtida, colando-se em cruz os bordos dos cortes indicados na figura 3.6. Homeomorficamente fazemos isso sobre as esferas cortadas $\mathbb{C} \cup \infty$, rotacionando de modo que o bordo + cole com o - como na figura 3.8 


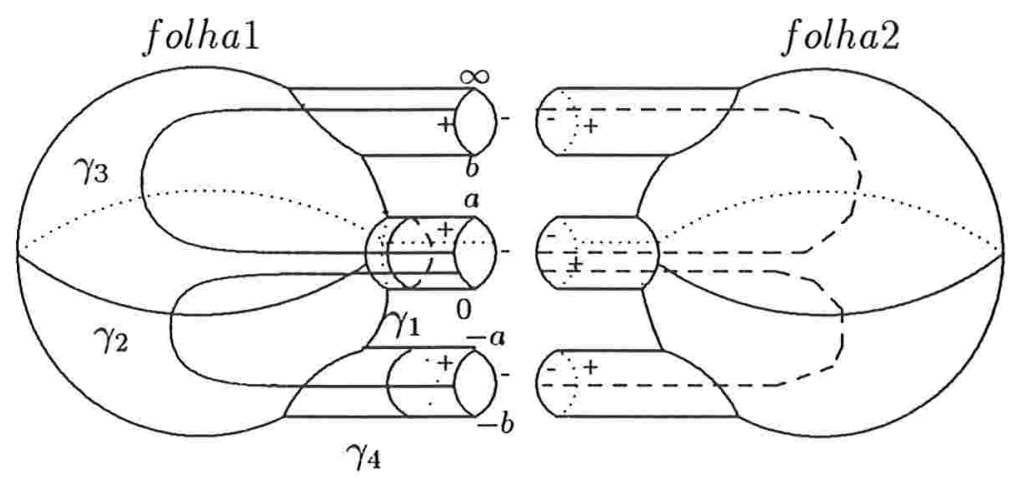

Figura 3.8

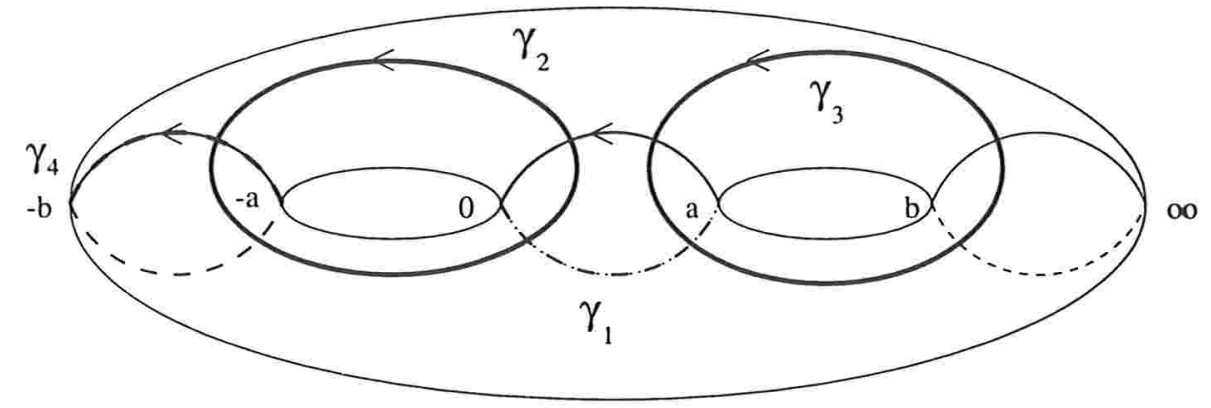

Figura 3.9

As curvas indicadas por $\gamma_{1}, \gamma_{2}, \gamma_{3}$ e $\gamma_{4}$ nas figuras 3.6 e 3.9 , formam uma base para a homologia de $\bar{R}$, que tem gênero $p=2$.

Demonstração de $c_{1}$ :

No que segue analisaremos o comportamento de $g$ e $\eta$ nas vizinhanças dos pontos de ramificação; $-b,-a, 0, a, b$ e $z=\infty$ de $w(z)$. Seja $P_{0}$ um ponto regular da superfície de Riemann $\bar{R}, z: \bar{R} \longrightarrow \mathbb{C} \cup\{\infty\}$ um sistema de coordenadas tal que $z\left(P_{0}\right)=z_{0} \in \mathbb{C} \cup\{\infty\}$ e $z_{0} \neq-b,-a, 0, a, b$ e $\infty$ graficamente a situação é descrita na figura 3.10 


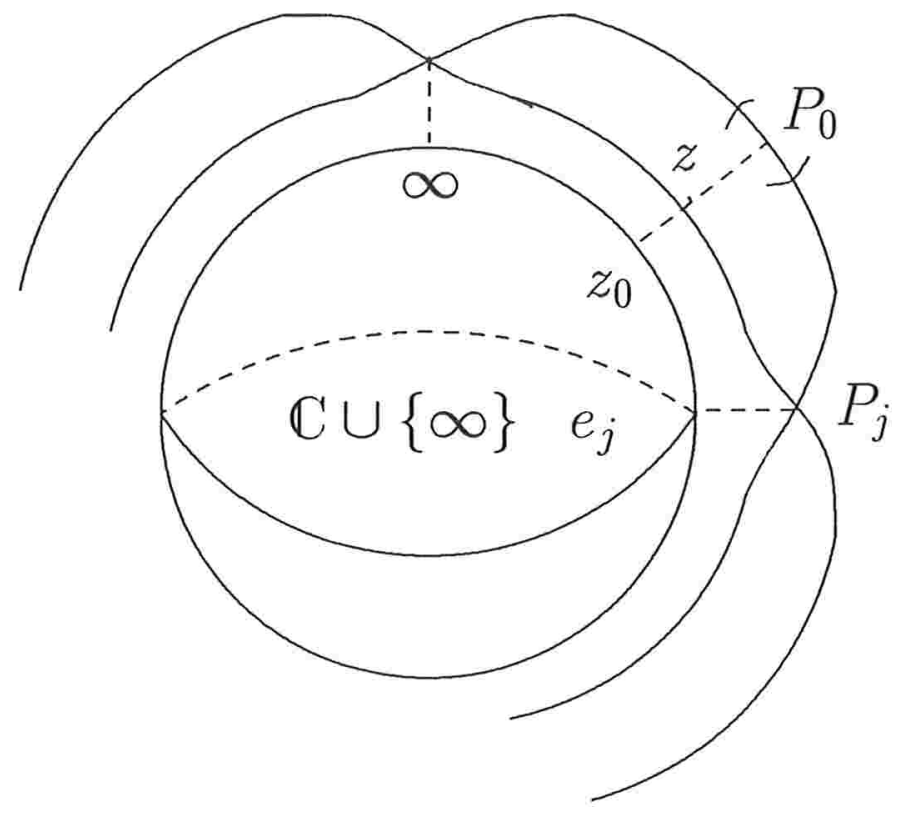

Figura 3.10

Então, neste caso podemos escolher como coordenadas locais $\zeta=z-z_{0}$. Logo:

$$
\tilde{g}(\zeta)=g\left(\zeta+z_{0}\right)=B \cdot \frac{\sqrt{\left(\zeta+z_{0}\right)\left(\zeta+z_{0}-a\right)\left(\zeta+z_{0}+a\right)\left(\zeta+z_{0}-b\right)\left(\zeta+z_{0}+b\right)}}{\left(\zeta+z_{0}-a\right)\left(\zeta+z_{0}+a\right)}
$$

Na vizinhança de $\zeta=0$, que corresponde a $z=z_{0}, \widetilde{g}(\zeta)$ é holomorfa.

$$
\tilde{\eta}(\zeta)=\eta\left(\zeta+z_{0}\right)=\frac{\left(\zeta+z_{0}-a\right)\left(\zeta+z_{0}+a\right)}{\sqrt{\left(\zeta+z_{0}\right)\left(\zeta+z_{0}-a\right)\left(\zeta+z_{0}+a\right)\left(\zeta+z_{0}-b\right)\left(\zeta+z_{0}+b\right)}} d \zeta
$$

também na vizinhança de $\zeta=0 \quad \tilde{\eta}(\zeta)$ é holomorfa. Portanto, $g$ e $\eta$ o são em $z_{0}$.

Se $z\left(P_{j}\right)=e_{j}$, onde $e_{j}$ é um dos pontos de ramificação, distinto do ponto de ramificação correspondente ao infinito, a coordenada local é $\zeta=\sqrt{z-e_{j}}$. Examinemos o caso em que $e_{j}=-b$. Temos, $z=\zeta^{2}-b, d z=2 \zeta d \zeta$,

$$
\widetilde{g}(\zeta)=B \cdot \frac{\sqrt{\left(\zeta^{2}-b\right)\left(\zeta^{2}-b-a\right)\left(\zeta^{2}-b+a\right)\left(\zeta^{2}-2 b\right) \zeta^{2}}}{\left(\zeta^{2}-b-a\right)\left(\zeta^{2}-b+a\right)}=
$$




$$
=\zeta \cdot B \cdot \frac{\sqrt{\left(\zeta^{2}-b\right)\left(\zeta^{2}-b-a\right)\left(\zeta^{2}-b+a\right)\left(\zeta^{2}-2 b\right)}}{\left(\zeta^{2}-b-a\right)\left(\zeta^{2}-b+a\right)} .
$$

Logo $\tilde{g}(\zeta)$, em $\zeta=0$, tem um zero simples, isto é, $g(\zeta)$ tem um zero simples em $-b$, pois $0<a<b$. Na vizinhança do mesmo ponto vejamos o que acontece $\operatorname{com} \eta(z)$,

$$
\begin{aligned}
\tilde{\eta}(\zeta) & =\frac{\left(\zeta^{2}-b-a\right)\left(\zeta^{2}-b+a\right)}{\sqrt{\left(\zeta^{2}-b\right)\left(\zeta^{2}-b-a\right)\left(\zeta^{2}-b+a\right)\left(\zeta^{2}-2 b\right) \zeta^{2}}} 2 \zeta d \zeta= \\
& =2 \frac{\left(\zeta^{2}-b-a\right)\left(\zeta^{2}-b+a\right)}{\sqrt{\left(\zeta^{2}-b\right)\left(\zeta^{2}-b-a\right)\left(\zeta^{2}-b+a\right)\left(\zeta^{2}-2 b\right)}} d \zeta,
\end{aligned}
$$

que é holomorfa em $\zeta=0$.

Se $e_{j}=-a$, então $z=\zeta^{2}-a$ e $d z=2 \zeta d \zeta$. Temos,

$$
\begin{aligned}
\tilde{g}(\zeta) & =B \cdot \frac{\sqrt{\left(\zeta^{2}-a\right)\left(\zeta^{2}-2 a\right) \zeta^{2}\left(\zeta^{2}-a-b\right)\left(\zeta^{2}-a+b\right)}}{\left(\zeta^{2}-2 a\right) \zeta^{2}}= \\
& =\frac{1}{\zeta} B \frac{\sqrt{\left(\zeta^{2}-a\right)\left(\zeta^{2}-2 a\right)\left(\zeta^{2}-a-b\right)\left(\zeta^{2}-a+b\right)}}{\left(\zeta^{2}-2 a\right)}, \quad \text { e } \\
\tilde{\eta}(\zeta) & =\frac{\left(\zeta^{2}-2 a\right) \zeta^{2}}{\sqrt{\left(\zeta^{2}-a\right)\left(\zeta^{2}-2 a\right) \zeta^{2}\left(\zeta^{2}-a-b\right)\left(\zeta^{2}-a+b\right)}} 2 \zeta d \zeta= \\
& =\zeta^{2} \cdot \frac{2\left(\zeta^{2}-2 a\right)}{\sqrt{\left(\zeta^{2}-a\right)\left(\zeta^{2}-2 a\right)\left(\zeta^{2}-a-b\right)\left(\zeta^{2}-a+b\right)}} d \zeta
\end{aligned}
$$

Então, na vizinhança de $\zeta=0 \tilde{g}(\zeta)$ e $\tilde{\eta}(\zeta)$ têm respectivamente um polo simples e um zero de ordem 2. Logo $g(z)$ tem a um polo simples em $z=-a$ e $\eta(z)$ um zero duplo nesse ponto.

Se $e_{j}=0$, então $z=\zeta^{2}$ e $d z=2 \zeta d \zeta$, logo na vizinhança de $\zeta=0$,

$$
\begin{aligned}
\tilde{g}(\zeta) & =B \cdot \frac{\sqrt{\zeta^{2}\left(\zeta^{2}-a\right)\left(\zeta^{2}+a\right)\left(\zeta^{2}-b\right)\left(\zeta^{2}+b\right)}}{\left(\zeta^{2}-a\right)\left(\zeta^{2}+a\right)}= \\
& =\zeta \cdot B \frac{\sqrt{\left(\zeta^{2}-a\right)\left(\zeta^{2}+a\right)\left(\zeta^{2}-b\right)\left(\zeta^{2}+b\right)}}{\left(\zeta^{2}-a\right)\left(\zeta^{2}+a\right)}, \quad \mathrm{e}
\end{aligned}
$$




$$
\begin{aligned}
\tilde{\eta}(\zeta) & =\frac{\left(\zeta^{2}-a\right)\left(\zeta^{2}+a\right)}{\sqrt{\zeta^{2}\left(\zeta^{2}-a\right)\left(\zeta^{2}+a\right)\left(\zeta^{2}-b\right)\left(\zeta^{2}+b\right)}} 2 \zeta d \zeta= \\
& =2 \frac{\left(\zeta^{2}-a\right)\left(\zeta^{2}+a\right)}{\sqrt{\left(\zeta^{2}-a\right)\left(\zeta^{2}+a\right)\left(\zeta^{2}-b\right)\left(\zeta^{2}+b\right)}} d \zeta,
\end{aligned}
$$

Logo, $\zeta=0$ é um zero simples de $\widetilde{g}(\zeta)$ e um ponto regular de $\widetilde{\eta}$, por conseguinte $z=0$ é um zero de $g(z)$ e um ponto regular para $\eta(z)$.

No caso $e_{j}=a$, seja $\zeta=\sqrt{z-a}$, então $d z=2 \zeta d \zeta$, analogamente ao caso $e_{j}=-a$, temos

$$
\begin{gathered}
\widetilde{g}(\zeta)=\frac{1}{\zeta} \cdot \frac{B \sqrt{\left(\zeta^{2}+a\right)\left(\zeta^{2}+2 a\right)\left(\zeta^{2}+a-b\right)\left(\zeta^{2}+a+b\right)}}{\left(\zeta^{2}+2 a\right)} \mathrm{e} \\
\widetilde{\eta}(\zeta)=\zeta^{2} \frac{2\left(\zeta^{2}+2 a\right)}{\sqrt{\left(\zeta^{2}+a\right)\left(\zeta^{2}+2 a\right)\left(\zeta^{2}+a-b\right)\left(\zeta^{2}+a+b\right)}} d \zeta .
\end{gathered}
$$

Como $0<a<b$, temos que em $z=a, g(z)$ tem um polo simples e $\eta(z)$ tem um zero de ordem 2 .

Se $e_{j}=b$, os cálculos são semelhantes ao caso em que $e_{j}=-b$. Então em $z=b$, $g(z)$ tem um zero simples e $\eta(z)$ é regular nesse ponto.

Por último analisemos qual o comportamento de $g(z)$ e $\eta(z)$ na vizinhança de $z=\infty$. A coordenada local do ponto de ramifização $e_{j}=\infty$ é $\zeta=\frac{1}{\sqrt{z}}$. Então: $d z=\frac{-2}{\zeta^{3}} d \zeta$ e $z=\frac{1}{\zeta^{2}}$. E portanto,

$$
\begin{aligned}
\tilde{g}(\zeta) & =\frac{B \sqrt{\frac{1}{\zeta^{2}}\left(\frac{1}{\zeta^{2}}-a\right)\left(\frac{1}{\zeta^{2}}+a\right)\left(\frac{1}{\zeta^{2}}-b\right)\left(\frac{1}{\zeta^{2}}+b\right)}}{\left(\frac{1}{\zeta^{2}}-a\right)\left(\frac{1}{\zeta^{2}}+a\right)}= \\
& =\frac{1}{\zeta} \frac{B \sqrt{\left(1-\zeta^{2} a\right)\left(1+\zeta^{2} a\right)\left(1-\zeta^{2} b\right)\left(1+\zeta^{2} b\right)}}{\left(1-\zeta^{2} a\right)\left(1+\zeta^{2} a\right)}, \quad \text { e } \\
\widetilde{\eta}(\zeta) & =\zeta^{-4} \frac{\left(1-\zeta^{2} a\right)\left(1+\zeta^{2} a\right)}{\zeta^{-5} \sqrt{\left(1-\zeta^{2} a\right)\left(1+\zeta^{2} a\right)\left(1-\zeta^{2} b\right)\left(1+\zeta^{2} b\right)}}-2 \zeta^{-3} d \zeta= \\
& =\frac{1}{\zeta^{2}} \frac{-2\left(1-\zeta^{2} a\right)\left(1+\zeta^{2} a\right)}{\sqrt{\left(1-\zeta^{2} a\right)\left(1+\zeta^{2} a\right)\left(1-\zeta^{2} b\right)\left(1+\zeta^{2} b\right)}} d \zeta .
\end{aligned}
$$


Logo em $\zeta=0, \widetilde{g}(\zeta)$ tem um polo simples e $\widetilde{\eta}(\zeta)$ tem um polo de ordem 2. E, por conseguinte, $z=0$ é um polo simples de $g(z)$ e um polo duplo de $\eta(z)$. Portanto, se exitir a tal superfície $S$ este ponto é um "candidato" a fim. Os resultados acima estão resumidos na tabela a seguir.

\begin{tabular}{|c|c|c|c|c|c|c|}
\hline$z$ & $-b$ & $-a$ & 0 & $a$ & $b$ & $\infty$ \\
\hline$g(z)$ & 0 & $\infty$ & 0 & $\infty$ & 0 & $\infty$ \\
\hline$\eta(z)$ & $*$ & $0^{2}$ & $*$ & $0^{2}$ & $*$ & $\infty^{2}$ \\
\hline $\mathrm{S}$ & $\begin{array}{c}\text { ponto } \\
\text { regular }\end{array}$ & $\begin{array}{c}\text { ponto } \\
\text { regular }\end{array}$ & $\begin{array}{c}\text { ponto } \\
\text { regular }\end{array}$ & $\begin{array}{c}\text { ponto } \\
\text { regular }\end{array}$ & $\begin{array}{c}\text { ponto } \\
\text { regular }\end{array}$ & $\begin{array}{c}\text { fim de ordem } \\
k_{1}=3\end{array}$ \\
\hline
\end{tabular}

Se existe a superfíce mínima $S$, isto é, se as condições $\mathbf{c}_{\mathbf{1}}, \mathbf{c}_{2}, \mathbf{c}_{\mathbf{3}}$ e $\mathbf{c}_{\mathbf{4}}$ do teorema 1.5.21 são satisfeitas, então $S$ será conformemente equivalente à $\bar{R} \backslash\{\infty\}$. Assim sendo, teremos $p=2, N=1$ e $\operatorname{grau}(g)=3$. Logo $C(S)=-4 \pi \cdot \operatorname{grau}(g)=-12 \pi$. Segue-se da fórmula de Jorge-Meeks-Gackstätter, dada em 1.5.19, que:

$-12 \pi=2 \pi\left(\chi(R)-k_{1}\right)=2 \pi\left(2-2 p-N-k_{1}\right)=2 \pi\left(2-2.2-1-k_{1}\right)=2 \pi\left(-3-k_{1}\right) \Rightarrow k_{1}=3$

Logo o fim não é mergulhado.

Da tabela acima, é fácil ver que $\eta(z)$ é holomorfa em $R=\bar{R} \backslash\{\infty\}$, e nos pontos em que $g$ tem polo simples, $\eta(z)$ tem zero de ordem 2 , portanto a condição $\mathrm{c}_{1}$ é satisfeita.

\section{Demonstração de $c_{2}$ :}

Para calcular os períodos, precisamos analisar doze integrais:

$$
\int_{\gamma_{\nu}} \phi_{j}(z), j=1,2,3 \quad \text { e } \quad \nu=1,2,3,4
$$

onde $\gamma_{1}, \gamma_{2}, \gamma_{3}$ e $\gamma_{4}$ são as curvas fechadas $\gamma_{\nu}:[0,1) \longrightarrow \mathbb{C}$ descritas na figura 3.6

Escrevendo, explicitamente, as expressões de $\phi_{1}, \phi_{2}$ e $\phi_{3}$ temos: 


$$
\begin{aligned}
\phi_{1}(z) & =\frac{1}{2} f\left(1-g^{2}\right) d z=\frac{1}{2} \frac{\left(z^{2}-a^{2}\right)}{w(z)}\left(1-\frac{B^{2} w(z)^{2}}{\left(z^{2}-a^{2}\right)^{2}}\right) d z \\
& =\frac{1}{2} \frac{\left(z^{2}-a^{2}\right)}{w(z)}\left(1-\frac{B^{2} z\left(z^{2}-b^{2}\right)}{\left(z^{2}-a^{2}\right)}\right) d z \\
& =\frac{1}{2}\left[\frac{\left(z^{2}-a^{2}\right)-B^{2} z\left(z^{2}-b^{2}\right)}{w(z)}\right] d z \\
\emptyset_{1}(z)= & \frac{1}{2}\left[\frac{\left(z^{2}-a^{2}\right)-B^{2} z\left(z^{2}-b^{2}\right)}{\left.\sqrt{z\left(z^{2}-a^{2}\right)\left(z^{2}-b^{2}\right)}\right] d z}\right. \\
\oint_{2}(z)= & \frac{i}{2} f\left(1+g^{2}\right) d z=\frac{1}{2} \frac{\left(z^{2}-a^{2}\right)}{w(z)}\left(1+\frac{B^{2} w(z)^{2}}{\left(z^{2}-a^{2}\right)^{2}}\right) d z \\
= & \frac{i}{2} \frac{\left(z^{2}-a^{2}\right)}{w(z)}\left(1+\frac{B^{2} z\left(z^{2}-b^{2}\right)}{\left(z^{2}-a^{2}\right)}\right) d z \\
= & \frac{i}{2}\left[\frac{\left(z^{2}-a^{2}\right)+B^{2} z\left(z^{2}-b^{2}\right)}{w(z)}\right] d z \\
\phi_{2}(z)= & \frac{i}{2}\left[\frac{\left(z^{2}-a^{2}\right)+B^{2} z\left(z^{2}-b^{2}\right)}{\left.\sqrt{z\left(z^{2}-a^{2}\right)\left(z^{2}-b^{2}\right)}\right]} d z\right. \\
\emptyset_{3}(z)= & g f d z=\left[\frac{\left(z^{2}-a^{2}\right)}{w(z)} \cdot \frac{B w(z)}{\left(z^{2}-a^{2}\right)}\right] d z=B d z
\end{aligned}
$$

Obviamente. nào existe problema para anular a parte real do período de $\phi_{3}$, já que

$$
\int_{\gamma_{\nu}} \phi_{3}(z)=\int_{\gamma_{\nu}} B d z=B[z]_{\gamma_{\nu}(0)}^{\gamma_{\nu}(1)}=B\left(\gamma_{\nu}(1)-\gamma_{\nu}(0)\right)=0
$$

Se $z=x+i y, x, y \in \mathbb{R}$, então a terceira coordenada da imersão $X: R \longrightarrow \mathbb{R}^{3}$ se existir é $x_{3}=B x$. Restam "apenas" oito períodos a serem analisados; o primeiro é $\int_{\gamma_{1}} \phi_{1}(z)$. Vale ressaltar que as curvas da figura 3.6 são homotópicas as da figura abaixo. Os períodos calculados naquelas curvas são os mesmos sobre as curvas da figura 3.11 e 3.12 indicadas abaixo. 


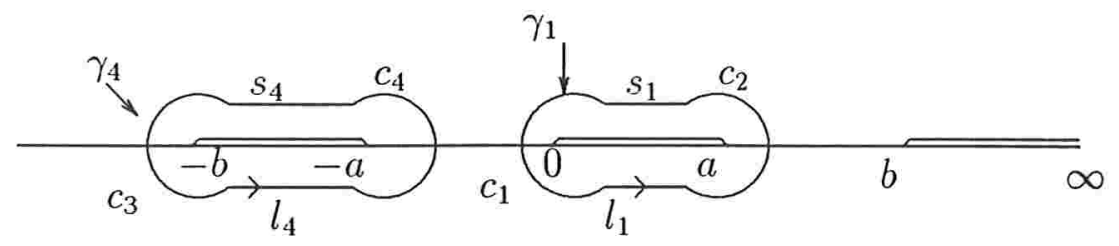

Figura 3.11

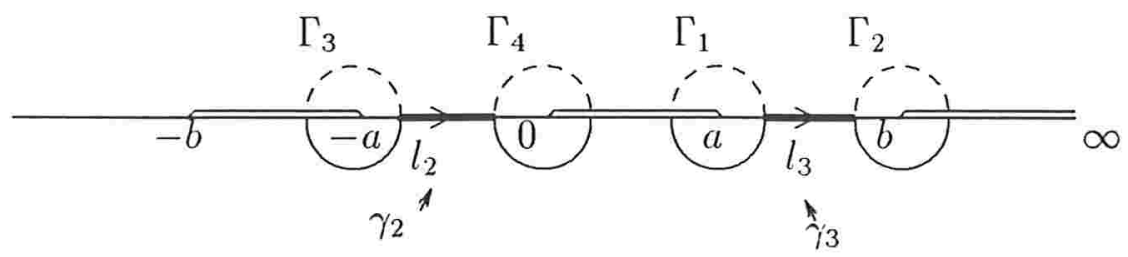

Figura 3.12

Onde, $c_{1}: z=\Sigma e^{i t}, t \in[0,2 \pi], c_{2}: z=a+\varepsilon e^{i t}, t \in[-\pi, \pi], c_{3}: z=-b+\varepsilon e^{i t}, t \in$ $[0,2 \pi], c_{4}: z=-a+\varepsilon e^{i t}, t \in[-\pi, \pi], l_{2}:-a+\varepsilon \leq x \leq-\varepsilon$ e $l_{3}: a+\varepsilon \leq x \leq b-\varepsilon$. E $\Gamma_{1}, \Gamma_{2}, \Gamma_{3}, \Gamma_{4}$ são semelhantes a $c_{1}, c_{2}, c_{3}$ e $c_{4}$, só que passam pelas duas folhas de $w(z)$. Então,

$$
\int_{\gamma_{1}} \phi_{1}(z)=\int_{c_{1}} \phi_{1}(z)+\int_{l_{1}} \phi_{1}(x)+\int_{c_{2}} \phi_{1}(z)+\int_{s_{1}} \phi_{1}(x)
$$

Quando $\varepsilon \rightarrow 0$, pelo lema 3.2.2 ítem i) e ii) temos que

$$
\operatorname{Re}\left\{\int_{\gamma_{1}} \phi_{1}(z)\right\}=\int_{\gamma_{1}} \phi_{1}(z)=\int_{l_{1}} \phi_{1}(x)+\int_{s_{1}} \phi_{1}(x)
$$

Escolhendo um ramo de $w(z)=\sqrt{z\left(z^{2}-a^{2}\right)\left(z^{2}-b^{2}\right)}$ definida sobre $\mathbb{C} \backslash\{[-b,-a] \cup[0, a] \cup[a, \infty)\}$, pertencente a uma das $j=1,2$ folhas da superfície de Riemann compacta $\bar{R}$, de modo que, quando $w(z)$ esteja na parte superior dos cortes das folhas $j=1,2, \arg (w(z))=\pi j$. Por exemplo, na parte superior do corte $[0, a]$, $w(z)=e^{j \pi i} \sqrt{z\left(z^{2}-a^{2}\right)\left(z^{2}-b^{2}\right)}$. Portanto, 


$$
\begin{aligned}
\int_{\gamma_{1}} \phi_{1}(z) & =\frac{1}{2} \int_{0}^{a}\left[\frac{\left(x^{2}-a^{2}\right)-B^{2} x\left(x^{2}-b^{2}\right)}{\sqrt{x\left(x^{2}-a^{2}\right)\left(x^{2}-b^{2}\right)}}\right] d x+\frac{1}{2} \int_{a}^{0}\left[\frac{\left(x^{2}-a^{2}\right)-B^{2} x\left(x^{2}-b^{2}\right)}{e^{\pi i} \sqrt{x\left(x^{2}-a^{2}\right)\left(x^{2}-b^{2}\right)}}\right] d x \\
\int_{\gamma_{1}} \phi_{1}(z) & =\frac{1}{2} \int_{0}^{a}\left[\frac{\left(x^{2}-a^{2}\right)-B^{2} x\left(x^{2}-b^{2}\right)}{\sqrt{x\left(x^{2}-a^{2}\right)\left(x^{2}-b^{2}\right)}}\right] d x+\frac{1}{2} \int_{0}^{a}\left[\frac{\left(x^{2}-a^{2}\right)-B^{2} x\left(x^{2}-b^{2}\right)}{\sqrt{x\left(x^{2}-a^{2}\right)\left(x^{2}-b^{2}\right)}}\right] d x \\
\int_{\gamma_{1}} \phi_{1}(z) & =\int_{0}^{a} \frac{\left(x^{2}-a^{2}\right)}{\sqrt{x\left(x^{2}-a^{2}\right)\left(x^{2}-b^{2}\right)}} d x-B^{2} \int_{0}^{a} \frac{x\left(x^{2}-b^{2}\right)}{\sqrt{x\left(x^{2}-a^{2}\right)\left(x^{2}-b^{2}\right)}} d x \\
\int_{\gamma_{1}} \phi_{1}(z) & =-\int_{0}^{a} \frac{\left(a^{2}-x^{2}\right)}{\sqrt{x\left(a^{2}-x^{2}\right)\left(b^{2}-x^{2}\right)}} d x+B^{2} \int_{0}^{a} \frac{x\left(b^{2}-x^{2}\right)}{\sqrt{x\left(a^{2}-x^{2}\right)\left(b^{2}-x^{2}\right)}} d x
\end{aligned}
$$

Pondo,

$$
\begin{aligned}
& F_{1}=F_{1}(a, b):=\int_{0}^{a} \frac{\left(a^{2}-x^{2}\right)}{\sqrt{x\left(a^{2}-x^{2}\right)\left(b^{2}-x^{2}\right)}} d x, \\
& F_{2}=F_{2}(a, b):=\int_{0}^{a} \frac{x\left(b^{2}-x^{2}\right)}{\sqrt{x\left(a^{2}-x^{2}\right)\left(b^{2}-x^{2}\right)}} d x,
\end{aligned}
$$

temos,

$$
\int_{\gamma_{1}} \phi_{1}(z)=-F_{1}+B^{2} F_{2} \in \mathbb{R}
$$

Observemos que $F_{1}>0$ e $F_{2}>0 \quad \forall x \in[0, a]$. Mostraremos, agora, que ambas integrais impróprias $F_{1}$ e $F_{2}$ convergem para um número finito.

$$
\begin{aligned}
& F_{1}=F_{1}(a, b)=\int_{0}^{a} \frac{\left(a^{2}-x^{2}\right)}{\sqrt{x\left(a^{2}-x^{2}\right)\left(b^{2}-x^{2}\right)}} d x \leq \sqrt{\frac{2 a}{\left(b^{2}-a^{2}\right)}} \int_{0}^{a} \sqrt{\frac{a-x}{x}} d x \\
& F_{1} \leq \sqrt{\frac{2 a}{\left(b^{2}-a^{2}\right)}} \sqrt{a} \int_{0}^{a} \frac{1}{\sqrt{x}} d x=2 a \sqrt{\frac{2 a}{\left(b^{2}-a^{2}\right)}}
\end{aligned}
$$

$F_{2}=F_{2}(a, b)=\int_{0}^{a} \frac{x\left(b^{2}-x^{2}\right)}{\sqrt{x\left(a^{2}-x^{2}\right)\left(b^{2}-x^{2}\right)}} d x \leq \sqrt{b(b+a)} \int_{0}^{a} \frac{1}{\sqrt{a-x}} d x=2 \sqrt{a b(b+a)}$

Agora em $\gamma_{2}$ temos,

$$
\int_{\gamma_{2}} \phi_{1}(z)=\int_{\Gamma_{3}} \phi_{1}(z)+\int_{l_{2}} \phi_{1}(x)+\int_{\Gamma_{4}} \phi_{1}(z)+\int_{l_{2}^{-}} \phi_{1}(x)
$$


Quando $\varepsilon \rightarrow 0$, pelo lema 3.2.2 ítem (vii) e (viii) temos que

$$
\int_{\gamma_{2}} \phi_{1}(z)=\int_{l_{2}} \phi_{1}(x)+\int_{l_{2}^{-}} \phi_{1}(x)
$$

$$
\begin{aligned}
\int_{\gamma_{2}} \phi_{1}(z) & =\frac{1}{2} \int_{-a}^{0}\left[\frac{\left(x^{2}-a^{2}\right)-B^{2} x\left(x^{2}-b^{2}\right)}{\sqrt{x\left(x^{2}-a^{2}\right)\left(x^{2}-b^{2}\right)}}\right] d x+\frac{1}{2} \int_{0}^{-a}\left[\frac{\left(x^{2}-a^{2}\right)-B^{2} x\left(x^{2}-b^{2}\right)}{e^{\pi i} \sqrt{x\left(x^{2}-a^{2}\right)\left(x^{2}-b^{2}\right)}}\right] d x \\
& =-\frac{1}{2} \int_{0}^{-a} \frac{\left(x^{2}-a^{2}\right)-B^{2} x\left(x^{2}-b^{2}\right)}{\sqrt{x\left(x^{2}-a^{2}\right)\left(x^{2}-b^{2}\right)}} d x-\frac{1}{2} \int_{0}^{-a}\left[\frac{\left(x^{2}-a^{2}\right)-B^{2} x\left(x^{2}-b^{2}\right)}{\sqrt{x\left(x^{2}-a^{2}\right)\left(x^{2}-b^{2}\right)}}\right] d x \\
& =\frac{1}{2} \int_{0}^{a} \frac{\left(x^{2}-a^{2}\right)+B^{2} x\left(x^{2}-b^{2}\right)}{\sqrt{-x\left(x^{2}-a^{2}\right)\left(x^{2}-b^{2}\right)}} d x+\frac{1}{2} \int_{0}^{a} \frac{\left(x^{2}-a^{2}\right)+B^{2} x\left(x^{2}-b^{2}\right)}{\sqrt{-x\left(x^{2}-a^{2}\right)\left(x^{2}-b^{2}\right)}} d x \\
& =-\frac{i}{2} \int_{0}^{a} \frac{\left(x^{2}-a^{2}\right)+B^{2} x\left(x^{2}-b^{2}\right)}{\sqrt{x\left(x^{2}-a^{2}\right)\left(x^{2}-b^{2}\right)}} d x-\frac{i}{2} \int_{0}^{a} \frac{\left(x^{2}-a^{2}\right)+B^{2} x\left(x^{2}-b^{2}\right)}{\sqrt{x\left(x^{2}-a^{2}\right)\left(x^{2}-b^{2}\right)}} d x \\
& =i \int_{0}^{a} \frac{\left(a^{2}-x^{2}\right)}{\sqrt{x\left(a^{2}-x^{2}\right)\left(b^{2}-x^{2}\right)}} d x+i B^{2} \int_{0}^{a} \frac{x\left(b^{2}-x^{2}\right)}{\sqrt{x\left(a^{2}-x^{2}\right)\left(b^{2}-x^{2}\right)}} d x \\
\int_{\gamma_{2}} \phi_{1}(z) & =i F_{1}+i B^{2} F_{2} \in \mathbb{C}
\end{aligned}
$$

Agora vamos calcular o período,

$$
\int_{\gamma_{1}} \phi_{2}(z)=\int_{c_{1}} \phi_{2}(z)+\int_{l_{1}} \phi_{2}(x)+\int_{c_{2}} \phi_{2}(z)+\int_{s_{1}} \phi_{2}(x)
$$

Análogo ao caso $\int_{\gamma_{1}} \phi_{1}(z)$, segue pelo ítem $(i)$ e (ii) do lema 3.2 .2 que

$$
\int_{\gamma_{1}} \phi_{2}(z)=\int_{l_{1}} \phi_{2}(x)+\int_{s_{1}} \phi_{2}(x)
$$

Logo,

$$
\int_{\gamma_{1}} \phi_{2}(z)=\frac{i}{2} \int_{0}^{a}\left[\frac{\left(x^{2}-a^{2}\right)+B^{2} x\left(x^{2}-b^{2}\right)}{\sqrt{x\left(x^{2}-a^{2}\right)\left(x^{2}-b^{2}\right)}}\right] d x+\frac{i}{2} \int_{a}^{0}\left[\frac{\left(x^{2}-a^{2}\right)+B^{2} x\left(x^{2}-b^{2}\right)}{e^{\pi i} \sqrt{x\left(x^{2}-a^{2}\right)\left(x^{2}-b^{2}\right)}}\right] d x
$$




$$
\begin{aligned}
& =\frac{i}{2} \int_{0}^{a}\left[\frac{\left(x^{2}-a^{2}\right)+B^{2} x\left(x^{2}-b^{2}\right)}{\sqrt{x\left(x^{2}-a^{2}\right)\left(x^{2}-b^{2}\right)}}\right] d x+\frac{i}{2} \int_{0}^{a}\left[\frac{\left(x^{2}-a^{2}\right)+B^{2} x\left(x^{2}-b^{2}\right)}{\sqrt{x\left(x^{2}-a^{2}\right)\left(x^{2}-b^{2}\right)}}\right] d x \\
& =i \int_{0}^{a} \frac{\left(x^{2}-a^{2}\right)}{\sqrt{x\left(x^{2}-a^{2}\right)\left(x^{2}-b^{2}\right)}} d x+i B^{2} \int_{0}^{a} \frac{x\left(x^{2}-b^{2}\right)}{\sqrt{x\left(x^{2}-a^{2}\right)\left(x^{2}-b^{2}\right)}} d x \\
& =-i \int_{0}^{a} \frac{\left(a^{2}-x^{2}\right)}{\sqrt{x\left(a^{2}-x^{2}\right)\left(b^{2}-x^{2}\right)}} d x-i B^{2} \int_{0}^{a} \frac{x\left(b^{2}-x^{2}\right)}{\sqrt{x\left(a^{2}-x^{2}\right)\left(b^{2}-x^{2}\right)}} d x \\
\int_{\gamma_{1}} \phi_{2}(z) & =-i F_{1}-i B^{2} F_{2} \in \mathbb{C}
\end{aligned}
$$

Agora vamos calcular, $\int_{\gamma_{2}} \phi_{2}(z)$, pela figura 3.12 temos,

$$
\int_{\gamma_{2}} \phi_{2}(z)=\int_{\Gamma_{3}} \phi_{2}(z)+\int_{l_{2}} \phi_{2}(x)+\int_{\Gamma_{4}} o_{2}(z)+\int_{l_{2}^{-}} \phi_{2}(x)
$$

e, pelo lema 3.2 .2 ítem (vii) e (viii), segue-se

$$
\begin{gathered}
\int_{\gamma_{2}} \phi_{2}(z)=\int_{l_{2}} \phi_{2}(x)+\int_{l_{2}^{-}} \phi_{2}(x) \\
\int_{\gamma_{2}} \phi_{2}(z)=\frac{i}{2} \int_{-a}^{0}\left[\frac{\left(x^{2}-a^{2}\right)+B^{2} x\left(x^{2}-b^{2}\right)}{\sqrt{x\left(x^{2}-a^{2}\right)\left(x^{2}-b^{2}\right)}}\right] d x+\frac{i}{2} \int_{0}^{-a}\left[\frac{\left(x^{2}-a^{2}\right)+B^{2} x\left(x^{2}-b^{2}\right)}{e^{\pi i} \sqrt{x\left(x^{2}-a^{2}\right)\left(x^{2}-b^{2}\right)}}\right] d x \\
=-\frac{i}{2} \int_{0}^{-a} \frac{\left(x^{2}-a^{2}\right)+B^{2} x\left(x^{2}-b^{2}\right)}{\sqrt{x\left(x^{2}-a^{2}\right)\left(x^{2}-b^{2}\right)}} d x-\frac{i}{2} \int_{0}^{-a} \frac{\left(x^{2}-a^{2}\right)+B^{2} x\left(x^{2}-b^{2}\right)}{\sqrt{x\left(x^{2}-a^{2}\right)\left(x^{2}-b^{2}\right)}} d x \\
=\frac{i}{2} \int_{0}^{a} \frac{\left(x^{2}-a^{2}\right)-B^{2} x\left(x^{2}-b^{2}\right)}{\sqrt{-x\left(x^{2}-a^{2}\right)\left(x^{2}-b^{2}\right)}} d x+\frac{i}{2} \int_{0}^{a} \frac{\left(x^{2}-a^{2}\right)-B^{2} x\left(x^{2}-b^{2}\right)}{\sqrt{-x\left(x^{2}-a^{2}\right)\left(x^{2}-b^{2}\right)}} d x \\
=\frac{1}{2} \int_{0}^{a} \frac{\left(x^{2}-a^{2}\right)-B^{2} x\left(x^{2}-b^{2}\right)}{\sqrt{x\left(x^{2}-a^{2}\right)\left(x^{2}-b^{2}\right)}} d x+\frac{1}{2} \int_{0}^{a} \frac{\left(x^{2}-a^{2}\right)-B^{2} x\left(x^{2}-b^{2}\right)}{\sqrt{x\left(x^{2}-a^{2}\right)\left(x^{2}-b^{2}\right)}} d x \\
=-\int_{0}^{a} \frac{\left(a^{2}-x^{2}\right)}{\sqrt{x\left(a^{2}-x^{2}\right)\left(b^{2}-x^{2}\right)}} d x+B^{2} \int_{0}^{a} \frac{x\left(b^{2}-x^{2}\right)}{\sqrt{x\left(a^{2}-x^{2}\right)\left(b^{2}-x^{2}\right)}} d x \\
\int_{\gamma_{2}} \phi_{2}(z)=-F_{1}+B^{2} F_{2} \in \mathbb{R},
\end{gathered}
$$

Em resumo: 


\begin{tabular}{|c|c|c|}
\hline curvas & $\gamma_{1}$ & $\gamma_{2}$ \\
\hline $\int \phi_{1}(z)$ & $-F_{1}+B^{2} F_{2}$ & $i F_{1}+i B^{2} F_{2}$ \\
\hline $\int \phi_{2}(z)$ & $-i F_{1}-i B^{2} F_{2}$ & $-F_{1}+B^{2} F_{2}$ \\
\hline
\end{tabular}

A fim de encerrar os cálculos dos períodos, sejam $\gamma_{4}$ e $\gamma_{3}$ como nas figuras 3.11 e 3.12. Usando os ítens (iii) e (iv) do lema 3.2.2, temos

$$
\begin{aligned}
\int_{\gamma_{4}} \phi_{1}(z) & =\int_{l_{4}} \phi_{1}(x)+\int_{s_{4}} \phi_{1}(x) \\
& =\frac{1}{2} \int_{-b}^{-a} \frac{x^{2}-a^{2}-B^{2} x\left(x^{2}-b^{2}\right)}{\sqrt{x\left(x^{2}-a^{2}\right)\left(x^{2}-b^{2}\right)}} d x+\frac{1}{2} \int_{-a}^{-b} \frac{x^{2}-a^{2}-B^{2} x\left(x^{2}-b^{2}\right)}{e^{\pi i} \sqrt{x\left(x^{2}-a^{2}\right)\left(x^{2}-b^{2}\right)}} d x \\
& =-\frac{1}{2} \int_{-a}^{-b} \frac{x^{2}-a^{2}-B^{2} x\left(x^{2}-b^{2}\right)}{\sqrt{x\left(x^{2}-a^{2}\right)\left(x^{2}-b^{2}\right)}} d x-\frac{1}{2} \int_{-a}^{-b} \frac{x^{2}-a^{2}-B^{2} x\left(x^{2}-b^{2}\right)}{\sqrt{x\left(x^{2}-a^{2}\right)\left(x^{2}-b^{2}\right)}} d x \\
& =\frac{1}{2} \int_{a}^{b} \frac{x^{2}-a^{2}+B^{2} x\left(x^{2}-b^{2}\right)}{\sqrt{-x\left(x^{2}-a^{2}\right)\left(x^{2}-b^{2}\right)}} d x+\frac{1}{2} \int_{a}^{b} \frac{x^{2}-a^{2}+B^{2} x\left(x^{2}-b^{2}\right)}{\sqrt{-x\left(x^{2}-a^{2}\right)\left(x^{2}-b^{2}\right)}} d x \\
& =\frac{1}{2} \int_{a}^{b} \frac{x^{2}-a^{2}-B^{2} x\left(b^{2}-x^{2}\right)}{\sqrt{x\left(x^{2}-a^{2}\right)\left(b^{2}-x^{2}\right)}} d x+\frac{1}{2} \int_{a}^{b} \frac{x^{2}-a^{2}-B^{2} x\left(x^{2}-b^{2}\right)}{\sqrt{x\left(x^{2}-a^{2}\right)\left(b^{2}-x^{2}\right)}} d x \\
& =\int_{a}^{b} \frac{x^{2}-a^{2}}{\sqrt{x\left(x^{2}-a^{2}\right)\left(b^{2}-x^{2}\right)}} d x-B^{2} \int_{a}^{b} \frac{x\left(b^{2}-x^{2}\right)}{\sqrt{x\left(x^{2}-a^{2}\right)\left(b^{2}-x^{2}\right)}} d x
\end{aligned}
$$

A primeira igualdade é conseqüência do lema 3.2.2; a segunda, da definição do ramo de $w(z)$ e a quarta igualdade de uma simples mudança de variável $(x=-t)$. Introduzimos, agora, novos valores reais positivos:

$$
\begin{gathered}
F_{3}=F_{3}(a, b):=\int_{a}^{b} \frac{\left(x^{2}-a^{2}\right)}{\sqrt{x\left(x^{2}-a^{2}\right)\left(b^{2}-x^{2}\right)}} d x \leq \sqrt{\frac{b-a}{a}} \int_{a}^{b} \sqrt{\frac{1}{b-x}} d x \\
F_{3} \leq \sqrt{\frac{b-a}{a}} \int_{a}^{b} \frac{1}{\sqrt{b-x}} d x=\sqrt{\frac{b-a}{a}} 2 \sqrt{b-a}=\frac{2(b-a)}{\sqrt{a}} \\
F_{3} \geq \sqrt{\frac{a}{b^{2}(b-a)}} \int_{a}^{b} \sqrt{x-a} d x=\frac{2}{3} \sqrt{a}(b-a)>0
\end{gathered}
$$




$$
\begin{aligned}
F_{4}(a, b) & :=\int_{a}^{b} \frac{x\left(b^{2}-x^{2}\right)}{\sqrt{x\left(x^{2}-a^{2}\right)\left(b^{2}-x^{2}\right)}} d x \leq \sqrt{\frac{b^{2}(b-a)}{a}} \int_{a}^{b} \frac{1}{\sqrt{x-a}} d x=\frac{2 \sqrt{a} \cdot b(b-a)}{a} \\
F_{4} & =F_{4}(a, b) \geq \sqrt{\frac{a}{b-a}} \int_{a}^{b} \sqrt{b-x} d x=\sqrt{a}(b-a)>0
\end{aligned}
$$

Então:

$$
\int_{\gamma_{4}} \phi_{1}(z)=F_{3}-B^{2} F_{4} \in \mathbb{R}
$$

Analogamente,

$$
\begin{aligned}
\int_{\gamma_{4}} \phi_{2}(z) & =\int_{l_{4}} \phi_{2}(x)+\int_{s_{4}} \phi_{2}(x) \\
& =\frac{i}{2} \int_{-b}^{-a} \frac{x^{2}-a^{2}+B^{2} x\left(x^{2}-b^{2}\right)}{\sqrt{x\left(x^{2}-a^{2}\right)\left(x^{2}-b^{2}\right)}} d x+\frac{i}{2} \int_{-a}^{-b} \frac{x^{2}-a^{2}+B^{2} x\left(x^{2}-b^{2}\right)}{e^{\pi i} \sqrt{x\left(x^{2}-a^{2}\right)\left(x^{2}-b^{2}\right)}} d x \\
& =-\frac{i}{2} \int_{-a}^{-b} \frac{x^{2}-a^{2}+B^{2} x\left(x^{2}-b^{2}\right)}{\sqrt{x\left(x^{2}-a^{2}\right)\left(x^{2}-b^{2}\right)}} d x-\frac{i}{2} \int_{-a}^{-b} \frac{x^{2}-a^{2}+B^{2} x\left(x^{2}-b^{2}\right)}{\sqrt{x\left(x^{2}-a^{2}\right)\left(x^{2}-b^{2}\right)}} d x \\
& =\frac{i}{2} \int_{a}^{b} \frac{x^{2}-a^{2}-B^{2} x\left(x^{2}-b^{2}\right)}{\sqrt{-x\left(x^{2}-a^{2}\right)\left(x^{2}-b^{2}\right)}} d x+\frac{i}{2} \int_{a}^{b} \frac{x^{2}-a^{2}-B^{2} x\left(x^{2}-b^{2}\right)}{\sqrt{-x\left(x^{2}-a^{2}\right)\left(x^{2}-b^{2}\right)}} d x \\
& =\frac{i}{2} \int_{a}^{b} \frac{x^{2}-a^{2}+B^{2} x\left(b^{2}-x^{2}\right)}{\sqrt{x\left(x^{2}-a^{2}\right)\left(b^{2}-x^{2}\right)}} d x+\frac{i}{2} \int_{a}^{b} \frac{x^{2}-a^{2}+B^{2} x\left(b^{2}-x^{2}\right)}{\sqrt{x\left(x^{2}-a^{2}\right)\left(b^{2}-x^{2}\right)}} d x \\
& =i \int_{a}^{b} \frac{x^{2}-a^{2}}{\sqrt{x\left(x^{2}-a^{2}\right)\left(b^{2}-x^{2}\right)}} d x+i B^{2} \int_{a}^{b} \frac{x\left(b^{2}-x^{2}\right)}{\sqrt{x\left(x^{2}-a^{2}\right)\left(b^{2}-x^{2}\right)}} d x \\
\int_{\gamma_{4}} \phi_{2}(z) & =i F_{3}+i B^{2} F_{4} \in \mathbb{C}
\end{aligned}
$$

Por último falta calcular os períodos $\int_{\gamma_{3}} \phi_{1}(z)$ e $\int_{\gamma_{3}} \phi_{2}(z)$, o faremos sobre a curva homotópica à $\gamma_{3}$, como indicada na figura 3.12. Levando-se em consideração o $\arg (w(z))$ sobre $l_{3}$ e $l_{3}^{-}$, segue dos ítens $(v)$ e $(v i)$ do lema 3.2.2,

$$
\int_{\gamma_{3}} \phi_{1}(z)=\int_{l_{3}} \phi_{1}(x)+\int_{l_{3}^{-}} \phi_{1}(x)
$$




$$
\begin{aligned}
& =\frac{1}{2} \int_{a}^{b} \frac{x^{2}-a^{2}-B^{2} x\left(x^{2}-b^{2}\right)}{\sqrt{x\left(x^{2}-a^{2}\right)\left(x^{2}-b^{2}\right)}} d x+\frac{1}{2} \int_{b}^{a} \frac{x^{2}-a^{2}-B^{2} x\left(x^{2}-b^{2}\right)}{e^{\pi i} \sqrt{x\left(x^{2}-a^{2}\right)\left(x^{2}-b^{2}\right)}} d x \\
& =\int_{a}^{b} \frac{x^{2}-a^{2}}{\sqrt{x\left(x^{2}-a^{2}\right)\left(b^{2}-x^{2}\right)(-1)}} d x+B^{2} \int_{a}^{b} \frac{x\left(b^{2}-x^{2}\right)}{\sqrt{x\left(x^{2}-a^{2}\right)\left(b^{2}-x^{2}\right)(-1)}} d x \\
& =-i \int_{a}^{b} \frac{x^{2}-a^{2}}{\sqrt{x\left(x^{2}-a^{2}\right)\left(b^{2}-x^{2}\right)}} d x-i B^{2} \int_{a}^{b} \frac{x\left(b^{2}-x^{2}\right)}{\sqrt{x\left(x^{2}-a^{2}\right)\left(b^{2}-x^{2}\right)}} d x \\
\int_{\gamma_{3}} \phi_{1}(z) & =-i F_{3}-i B^{2} F_{4} \in \mathbb{C}
\end{aligned}
$$

Assim,

$$
\begin{aligned}
\int_{\gamma_{3}} \phi_{2}(z) & =\int_{l_{3}} \phi_{2}(x)+\int_{l_{3}^{-}} \phi_{2}(x) \\
& =\frac{i}{2} \int_{a}^{b} \frac{x^{2}-a^{2}+B^{2} x\left(x^{2}-b^{2}\right)}{\sqrt{x\left(x^{2}-a^{2}\right)\left(x^{2}-b^{2}\right)}} d x+\frac{i}{2} \int_{b}^{a} \frac{x^{2}-a^{2}+B^{2} x\left(x^{2}-b^{2}\right)}{e^{\pi i} \sqrt{x\left(x^{2}-a^{2}\right)\left(x^{2}-b^{2}\right)}} d x \\
& =i \int_{a}^{b} \frac{x^{2}-a^{2}}{\sqrt{x\left(x^{2}-a^{2}\right)\left(b^{2}-x^{2}\right)(-1)}} d x-i B^{2} \int_{a}^{b} \frac{\left.x x^{2}\right)}{\sqrt{x\left(x^{2}-a^{2}\right)\left(b^{2}-x^{2}\right)(-1)}} d x \\
& =\int_{a}^{b} \frac{x^{2}-a^{2}}{\sqrt{x\left(x^{2}-a^{2}\right)\left(b^{2}-x^{2}\right)}} d x-B^{2} \int_{a}^{b} \frac{x\left(b^{2}-x^{2}\right)}{\sqrt{x\left(x^{2}-a^{2}\right)\left(b^{2}-x^{2}\right)}} d x \\
\int_{\gamma_{3}} \phi_{2}(z) & =F_{3}-B^{2} F_{4} \in \mathbb{R}
\end{aligned}
$$

Resumindo os últimos resultados numa tabela temos:

\begin{tabular}{|c|c|c|}
\hline curvas & $\gamma_{3}$ & $\gamma_{4}$ \\
\hline $\int \phi_{1}(z)$ & $-i F_{3}-i B^{2} F_{4}$ & $F_{3}-B^{2} F_{4}$ \\
\hline $\int \phi_{2}(z)$ & $F_{3}-B^{2} F_{4}$ & $i F_{1}+i B^{2} F_{4}$ \\
\hline
\end{tabular}

Finalmente podemos agrupar os oito períodos numa só tabela (abaixo), permitindonos relacioná-los como descreveremos a seguir.

\begin{tabular}{|c|c|c|c|c|}
\hline curvas & $\gamma_{1}$ & $\gamma_{2}$ & $\gamma_{3}$ & $\gamma_{4}$ \\
\hline $\int \phi_{1}(z)$ & $-F_{1}+B^{2} F_{2}$ & $i F_{1}+i B^{2} F_{2}$ & $-i F_{3}-i B^{2} F_{4}$ & $F_{3}-B^{2} F_{4}$ \\
\hline $\int \phi_{2}(z)$ & $-i F_{1}-i B^{2} F_{2}$ & $-F_{1}+B^{2} F_{2}$ & $F_{3}-B^{2} F_{4}$ & $i F_{1}+i B^{2} F_{4}$ \\
\hline
\end{tabular}


Analisando a tabela acima, é fácil concluir que:

$$
\operatorname{Re}\left\{\int_{\gamma_{\nu}} \phi_{1}(z)\right\}=\operatorname{Re}\left\{\int_{\gamma_{k}} \phi_{2}(z)\right\}=0, \text { se } \nu=2,3 \text { e } k=1,4 \text {. }
$$

Então para condição $\mathrm{c}_{2}$ do teorema 1.5.21 ser satisfeita, basta provar o seguinte,

$$
\operatorname{Re}\left\{\int_{\gamma_{\nu}} \phi_{1}(z)\right\}=\operatorname{Re}\left\{\int_{\gamma_{k}} \phi_{2}(z)\right\}=0, \quad \text { se } \quad \nu=1,4 \quad \text { e } \quad k=2,3
$$

$$
\text { Isto é, } \quad-F_{1}+B^{2} F_{2}=0 \quad \text { e } \quad F_{3}-B^{2} F_{4}=0 \Leftrightarrow F_{1}=B^{2} F_{2} \quad \text { e } \quad F_{3}=B^{2} F_{4}
$$

Mostraremos. agora, a existência de $B$ real para o qual as duas últimas equações valham simultaneamente. Em resumo, devemos determinar $B$ tal que:

$$
\left\{\begin{array}{l}
F_{1} \cdot F_{4}=F_{2} \cdot F_{3} \\
F_{1}=B^{2} F_{2}
\end{array}\right.
$$

Pois, $\quad \frac{F_{1}}{F_{2}}=\frac{F_{3}}{F_{4}}=B^{2}$.

Podemos escolher um número real $\mathrm{B}$ adequado, a fim de que satisfaça sempre a equação $F_{1}=B^{2} F_{2}$ para qualquer valor de $a b$, para isso basta fazer $B=\sqrt{\frac{F_{1}}{F_{2}}}$. Nosso objetivo agora é mostrar que existem números reais $a$ e $b$, de tal modo que valha a primeira equação de 3.7 , isto é,

$$
\frac{F_{1}(a, b)}{F_{2}(a, b)}=\frac{F_{3}(a, b)}{F_{4}(a, b)} \text {. }
$$

Sem perda de generalidade podemos super que $a=1$ e $b>1$ (pois, sendo a transformação de Möbius $T(z):=z-a+1$, temos $T(a)=1$ e $T(b)>1)$. Sejam,

$$
\begin{aligned}
& L(b):=F_{1}(1, b) \cdot F_{4}(1, b)=\int_{0}^{1} \frac{1-x^{2}}{\sqrt{x\left(1-x^{2}\right)\left(b^{2}-x^{2}\right)}} d x \cdot \int_{1}^{b} \frac{x\left(b^{2}-x^{2}\right)}{\sqrt{x\left(x^{2}-1\right)\left(b^{2}-x^{2}\right)}} d x \quad \mathrm{e} \\
& H(b):=F_{2}(1, b) \cdot F_{3}(1, b)=\int_{0}^{1} \frac{x\left(b^{2}-x^{2}\right)}{\sqrt{x\left(1-x^{2}\right)\left(b^{2}-x^{2}\right)}} d x \cdot \int_{1}^{b} \frac{x^{2}-1}{\sqrt{x\left(x^{2}-1\right)\left(b^{2}-x^{2}\right)}} d x
\end{aligned}
$$

Então, o problema consiste em determinar o valor de $b>1$ para o qual $L(b)=H(b)$. Isto será feito com o auxílio do teorema do valor intermediário, mostrando que quando 
$b \rightarrow \infty, L(b) \leq H(b)$ e quando $b \rightarrow 1, L(b) \geq H(b)$. Com esse objetivo façamos a seguinte mudança de variável $y=x-1$ nas integrais:

$$
F_{4}(1, b)=\int_{1}^{b} \frac{x\left(b^{2}-x^{2}\right)}{\sqrt{x\left(x^{2}-1\right)\left(b^{2}-x^{2}\right)}} d x \quad \text { e } \quad F_{3}(1, b)=\int_{1}^{b} \frac{x^{2}-1}{\sqrt{x\left(x^{2}-1\right)\left(b^{2}-x^{2}\right)}} d x
$$

Temos :

$$
\begin{aligned}
& F_{4}=\int_{0}^{b-1} \frac{(y+1)\left(b^{2}-(y+1)^{2}\right)}{\sqrt{(y+1)\left((y+1)^{2}-1\right)\left(b^{2}-(y+1)^{2}\right)}} d y \quad \mathrm{e} \\
& F_{3}=\int_{0}^{b-1} \frac{(y+1)^{2}-1}{\sqrt{(y+1)\left((y+1)^{2}-1\right)\left(b^{2}-(y+1)^{2}\right)}} d y
\end{aligned}
$$

Logo,

$$
\begin{aligned}
& L(b)=\int_{0}^{1} \frac{1-x^{2}}{\sqrt{x\left(1-x^{2}\right)\left(b^{2}-x^{2}\right)}} d x \int_{0}^{b-1} \frac{(x+1)\left(b^{2}-(x+1)^{2}\right)}{\sqrt{(x+1)\left((x+1)^{2}-1\right)\left(b^{2}-(x+1)^{2}\right)}} d x= \\
& =\int_{0}^{1} \sqrt{\frac{\left(1-x^{2}\right)^{2}}{x\left(1-x^{2}\right)\left(b^{2}-x^{2}\right)}} d x \int_{0}^{b-1} \sqrt{\frac{(x+1)^{2}\left(b^{2}-(x+1)^{2}\right)^{2}}{(x+1)\left((x+1)^{2}-1\right)\left(b^{2}-(x+1)^{2}\right)}} d x= \\
& =\int_{0}^{1} \sqrt{\frac{(1+x)}{\left(b^{2}-x^{2}\right)}} \sqrt{\frac{1-x}{x}} d x \int_{0}^{b-1} \sqrt{\frac{(x+1)\left(b^{2}-(x+1)^{2}\right)}{x(x+2)}} d x \\
& L(b)=\int_{0}^{1} \sqrt{\frac{(1+x)}{(b+x)(b-x)}} \sqrt{\frac{1-x}{x}} d x \int_{0}^{b-1} \sqrt{\frac{(x+1)(b+x+1)}{x+2}} \sqrt{\frac{b-1-x}{x}} d x
\end{aligned}
$$

Já que estamos interessados em comparar $H(b)$ com $L(b)$, expressá-lo-emos de modo semelhante. Assim,

$$
\begin{aligned}
H(b) & =\int_{0}^{1} \sqrt{\frac{x^{2}\left(b^{2}-x^{2}\right)^{2}}{x\left(1-x^{2}\right)\left(b^{2}-x^{2}\right)}} d x \cdot \int_{0}^{b-1} \sqrt{\frac{\left((x+1)^{2}-1\right)^{2}}{(x+1)\left((x+1)^{2}-1\right)\left(b^{2}-(x+1)^{2}\right)}} d x= \\
& =\int_{0}^{1} \sqrt{\frac{x\left(b^{2}-x^{2}\right)}{\left(1-x^{2}\right)}} d x \cdot \int_{0}^{b-1} \sqrt{\frac{x(x+2)}{(x+1)\left(b^{2}-(x+1)^{2}\right)}} d x
\end{aligned}
$$

Fazendo duas mudanças de variáveis, $y=x-1$ e $y=x-(b-1)$ respectivamente nas integrais.

$$
\int_{0}^{1} \sqrt{\frac{x\left(b^{2}-x^{2}\right)}{\left(1-x^{2}\right)}} d x \quad \text { e } \quad \int_{0}^{b-1} \sqrt{\frac{x(x+2)}{(x+1)\left(b^{2}-(x+1)^{2}\right)}} d x, \text { temos }
$$




$$
\begin{aligned}
& \int_{0}^{1} \sqrt{\frac{x\left(b^{2}-x^{2}\right)}{\left(1-x^{2}\right)}} d x=\int_{0}^{1} \sqrt{\frac{(b-1+y)(b+1-y)}{2-y}} \cdot \sqrt{\frac{1-y}{y}} d y \quad \mathrm{e} \\
& \int_{0}^{b-1} \sqrt{\frac{x(x+2)}{(x+1)\left(b^{2}-(x+1)^{2}\right)}} d x=\int_{0}^{b-1} \sqrt{\frac{b+1-y}{(b-y)(2 b-y)}} \sqrt{\frac{b-1-y}{y}} d y
\end{aligned}
$$

Portanto,

$$
H(b)=\int_{0}^{1} \sqrt{\frac{(b-1+x)(b+1-x)}{2-x}} \sqrt{\frac{1-x}{x}} d x \cdot \int_{0}^{b-1} \sqrt{\frac{b+1-x}{(b-x)(2 b-x)}} \sqrt{\frac{b-1-x}{x}} d x
$$

Agora pelos ítens (i) (iii) do lema 3.2 .3 e equação 3.8 temos:

$$
\begin{aligned}
& L(b) \leq \int_{0}^{1} \sqrt{\frac{2}{b(b-1)}} \sqrt{\frac{1-x}{x}} d x \cdot \int_{0}^{b-1} \sqrt{2 b} \sqrt{\frac{b-1-x}{x}} d x \\
& L(b) \leq \sqrt{\frac{4}{b-1}}\left\{\int_{0}^{1} \sqrt{\frac{1-x}{x}} d x \cdot \int_{0}^{b-1} \sqrt{\frac{b-1-x}{x}} d x\right\}
\end{aligned}
$$

E pelos ítens (ii) (iv) do lema 3.2 .3 e equação 3.9 segue,

$$
\begin{aligned}
& H(b) \geq \int_{0}^{1} \sqrt{\frac{b(b-1)}{2}} \sqrt{\frac{1-x}{x}} d x . \int_{0}^{b-1} \sqrt{\frac{1}{2 b}} \sqrt{\frac{b-1-x}{x}} d x \\
& H(b) \geq \sqrt{\frac{b-1}{4}}\left\{\int_{0}^{1} \sqrt{\frac{1-x}{x}} d x \cdot \int_{0}^{b-1} \sqrt{\frac{b-1-x}{x}} d x\right\}
\end{aligned}
$$

Então, pelas inequações 3.10 e 3.11 temos:

$$
H(b) \geq \sqrt{\frac{b-1}{4}} \cdot \frac{L(b)}{\sqrt{\frac{4}{b-1}}} \quad \Leftrightarrow \quad H(b) \geq\left(\frac{b-1}{4}\right) L(b)
$$

Concluímos que para $b \rightarrow \infty$, segue $H(b) \geq L(b)$.

Agora para $b=1+\varepsilon, \varepsilon>0, \varepsilon \rightarrow 0$, a função $H(b) \leq L(b)$. De fato, para $\varepsilon$ suficientemente pequeno podemos aplicar o lema 3.2.4. Primeiro usando os ítens $(i)$ e (iii) em 3.8 obtemos,

$$
L(1+\varepsilon)=\int_{0}^{1} \sqrt{\frac{1+x}{1+\varepsilon+x}} \cdot \frac{1}{\sqrt{1+\varepsilon-x}} \sqrt{\frac{1-x}{x}} d x \int_{0}^{\varepsilon} \sqrt{\frac{(1+x)(2+\varepsilon+x)}{2+x}} \sqrt{\frac{\varepsilon-x}{x}} d x
$$




$$
\begin{aligned}
& L(1+\varepsilon) \geq \int_{0}^{1} \sqrt{\frac{0,81}{1+\varepsilon-x}} \sqrt{\frac{1-x}{x}} d x \int_{0}^{\varepsilon} \sqrt{1} \sqrt{\frac{\varepsilon-x}{x}} d x \\
& L(1+\varepsilon) \geq \int_{0}^{1} \frac{0,9}{\sqrt{1+\varepsilon-x}} \sqrt{\frac{1-x}{x}} d x \int_{0}^{\varepsilon} \sqrt{\frac{\varepsilon-x}{x}} d x
\end{aligned}
$$

Por último usando os ítens $(i i)$ e $(i v)$ do lema 3.2 .4 em 3.9 obtemos,

$$
\begin{gathered}
H(1+\varepsilon)=\int_{0}^{1} \sqrt{\frac{2+\varepsilon-x}{2-x}} \sqrt{\varepsilon+x} \sqrt{\frac{1-x}{x}} d x \int_{0}^{\varepsilon} \sqrt{\frac{2+\varepsilon-x}{(1+\varepsilon-x)(2+2 \varepsilon-x)}} \sqrt{\frac{\varepsilon-x}{x}} d x \\
H(1+\varepsilon) \leq \int_{0}^{1} \sqrt{1,21} \sqrt{\varepsilon+x} \cdot \sqrt{\frac{1-x}{x}} d x \int_{0}^{\varepsilon} \sqrt{1} \sqrt{\frac{\varepsilon-x}{x}} d x \\
H(1+\varepsilon) \leq \int_{0}^{1}(1,1) \sqrt{\varepsilon+x} \cdot \sqrt{\frac{1-x}{x}} d x \int_{0}^{\varepsilon} \sqrt{\frac{\varepsilon-x}{x}} d x
\end{gathered}
$$

Para poder comparar 3.12 e 3.13 só precisamos provar $\frac{0,9}{\sqrt{1+\varepsilon-x}} \geq(1,1) \sqrt{\varepsilon+x}$, o que já foi feito no ítem $(v)$ do lema 3.2.4. Logo,

$$
\begin{aligned}
& L(1+\varepsilon) \geq \int_{0}^{1} \frac{0,9}{\sqrt{1+\varepsilon-x}} \sqrt{\frac{1-x}{x}} d x \int_{0}^{\varepsilon} \sqrt{\frac{\varepsilon-x}{x}} d x \\
& H(1+\varepsilon) \leq \int_{0}^{1} \frac{0,9}{\sqrt{1+\varepsilon-x}} \cdot \sqrt{\frac{1-x}{x}} d x \int_{0}^{\varepsilon} \sqrt{\frac{\varepsilon-x}{x}} d x
\end{aligned}
$$

Portanto, $H(b) \leq L(b)$, quando $b \rightarrow 1$.

Agora definimos a função $f(b)=H(b)-L(b), \operatorname{logo} f(b) \geq 0$ quando $b \rightarrow \infty$, então existe $k>1$ tal que para todo $b \geq k, f(b) \geq 0$. E $f(b) \leq 0$ quando $b=1+\varepsilon$ e $\varepsilon \rightarrow 0$. Como $\left.f\right|_{[1+\varepsilon, k]}$ é contínua, pelo teorema do valor intermediário aplicado a $\left.f\right|_{[1+\varepsilon, k]}$, exite um número real $c \in(1+\varepsilon, k)$ tal que $f(c)=0$, isto é, $H(c)=L(c)$. Concluímos então que exite um número $c>1$, para o qual vale,

$$
\frac{F_{1}(1, c)}{F_{2}(1, c)}=\frac{F_{3}(1, c)}{F_{4}(1, c)}
$$

Demonstração de $c_{3}$ :

Aqui verificaremos que realmente os resíduos de $\phi_{j}, j=1,2,3$ no fim $P_{1}=\infty$ são 
reais. É óbvio que $\operatorname{Res}\left(\phi_{3} ; \infty\right)=0$, pois $\phi_{3}=B d z$. Usando a coordenada local $\zeta=\frac{1}{\sqrt{z}}, d z=\frac{-2}{\zeta^{3}} d \zeta$, em $\phi_{1}$ e $\phi_{2}$, temos:

$$
\begin{aligned}
\tilde{\phi}_{1}(\zeta):=\phi_{1}\left(\frac{1}{\zeta}\right) & =\frac{1}{2}\left\{\frac{\left(\frac{1}{\zeta^{4}}-a^{2}\right)-B^{2} \frac{1}{\zeta^{2}}\left(\frac{1}{\zeta^{4}}-b^{2}\right)}{\sqrt{\frac{1}{\zeta^{2}}\left(\frac{1}{\zeta^{4}}-a^{2}\right)\left(\frac{1}{\zeta^{4}}-b^{2}\right)}}\right\} \frac{-2}{\zeta^{3}} d \zeta= \\
& =\frac{-1}{\zeta^{4}}\left\{\frac{\zeta^{2}\left(1-\zeta^{4} a^{2}\right)-B^{2}\left(1-\zeta^{4} b^{2}\right)}{\sqrt{\left(1-\zeta^{4} a^{2}\right)\left(1-\zeta^{4} b^{2}\right)}} d \zeta\right\}
\end{aligned}
$$

Analogamente,

$$
\begin{aligned}
\tilde{\phi}_{2}(\zeta):=\phi_{2}\left(\frac{1}{\zeta}\right) & =\frac{i}{2}\left\{\frac{\left(\frac{1}{\zeta^{4}}-a^{2}\right)+B^{2} \frac{1}{\zeta^{2}}\left(\frac{1}{\zeta^{4}}-b^{2}\right)}{\sqrt{\frac{1}{\zeta^{2}}\left(\frac{1}{\zeta^{4}}-a^{2}\right)\left(\frac{1}{\zeta^{4}}-b^{2}\right)}}\right\} \frac{-2}{\zeta^{3}} d \zeta= \\
& =\frac{-i}{\zeta^{4}}\left\{\frac{\zeta^{2}\left(1-\zeta^{4} a^{2}\right)+B^{2}\left(1-\zeta^{4} b^{2}\right)}{\sqrt{\left(1-\zeta^{4} a^{2}\right)\left(1-\zeta^{4} b^{2}\right)}} d \zeta\right\}
\end{aligned}
$$

Logo, $\phi_{1}$ e $\phi_{2}$ têm um polo de ordem $4 \mathrm{em} z=\infty$, então $\operatorname{Res}\left(\phi_{1} ; \infty\right)=\operatorname{Res}\left(\phi_{2} ; \infty\right)=$ 0 . Portanto, acabamos de mostrar que existe a superfície mínima $S$ com as hipóteses do teorema, exceto a referente à completeza.

\section{Demonstração de $c_{4}$ :}

Seja $\gamma:[0,1) \longrightarrow \mathbb{C}$ uma curva divergente em $\bar{R} \backslash\{\infty\}$, com $\lim _{t \rightarrow 1^{-}}|\gamma(t)|=\infty$. Então,

$$
\operatorname{comp}(\gamma)=\int_{\gamma} \lambda(z)|d z|=\infty, \quad \text { onde } \quad \lambda(z)=\frac{1}{2}|f|\left(1+|g|^{2}\right) .
$$

De fato, como $\phi_{2}(z)$ tem um polo de ordem 4 em $z=\infty$, na vizinhança $V$ de $\zeta=0$, sendo $\zeta=\frac{1}{\sqrt{z}}$, podemos escrever $\tilde{\phi}_{2}(z)=\frac{1}{2 \zeta^{4}} h(\zeta) d \zeta$, onde $h(z)$ é uma função holomorfa e $h(0) \neq 0$ em $V$. Então, existe um número real $M>0$ tal que $|h(z)| \geq M, \quad \forall z \in U \subset V$. Logo para $\tilde{\gamma}(t):=\frac{1}{\gamma(t)}, \quad \lim _{t \rightarrow 1^{-}}|\tilde{\gamma}(t)|=0$ e $\tilde{\lambda}(\zeta)=\lambda\left(\frac{1}{\sqrt{z}}\right)$. Seja $\tilde{\gamma}(0)=\zeta_{0}$ e $\tilde{\gamma}(t)=\zeta$. Como,

$\left|\frac{1}{2 \zeta^{4}} h(\zeta) d \zeta\right|=\left|\tilde{\phi}_{2}(\zeta)\right|=\left|\frac{i}{2} f\left(1+g^{2}\right) d \zeta\right|=\frac{1}{2}|f|\left|1+g^{2}\right||d \zeta| \leq \frac{1}{2}|f|\left(1+|g|^{2}\right)|d \zeta|=\tilde{\lambda}(\zeta)|d \zeta|$

Então, $\quad \operatorname{comp} p(\tilde{\gamma})=\int_{\dot{\gamma}} \tilde{\lambda}(\zeta)|d \zeta| \geq \int_{\tilde{\gamma}}\left|\tilde{\phi}_{2}(\zeta)\right|=\frac{1}{2} \int_{\dot{\gamma}}\left|\frac{1}{\zeta^{4}} h(\zeta) d \zeta\right|=\frac{1}{2} \int_{\tilde{\gamma}}|h(\zeta)|\left|\frac{1}{\zeta^{4}} d \zeta\right|$ 
Assim, $\quad \operatorname{comp}(\tilde{\gamma}) \geq \frac{M}{2} \int_{\dot{\gamma}^{\prime}}\left|\frac{1}{\zeta^{4}} d \dot{\zeta}\right| \geq \frac{M}{2}\left|\int_{\bar{\gamma}} \frac{1}{\zeta^{4}} d \zeta\right|=\frac{M}{6}\left|\lim _{\zeta \rightarrow 0}\left(\frac{1}{\zeta^{3}}-\frac{1}{\zeta_{0}^{3}}\right)\right|=\infty$

Em [21] o matemático K. Sato. esboça o gráfico da superfície acima demonstrada, veja figura abaixo.

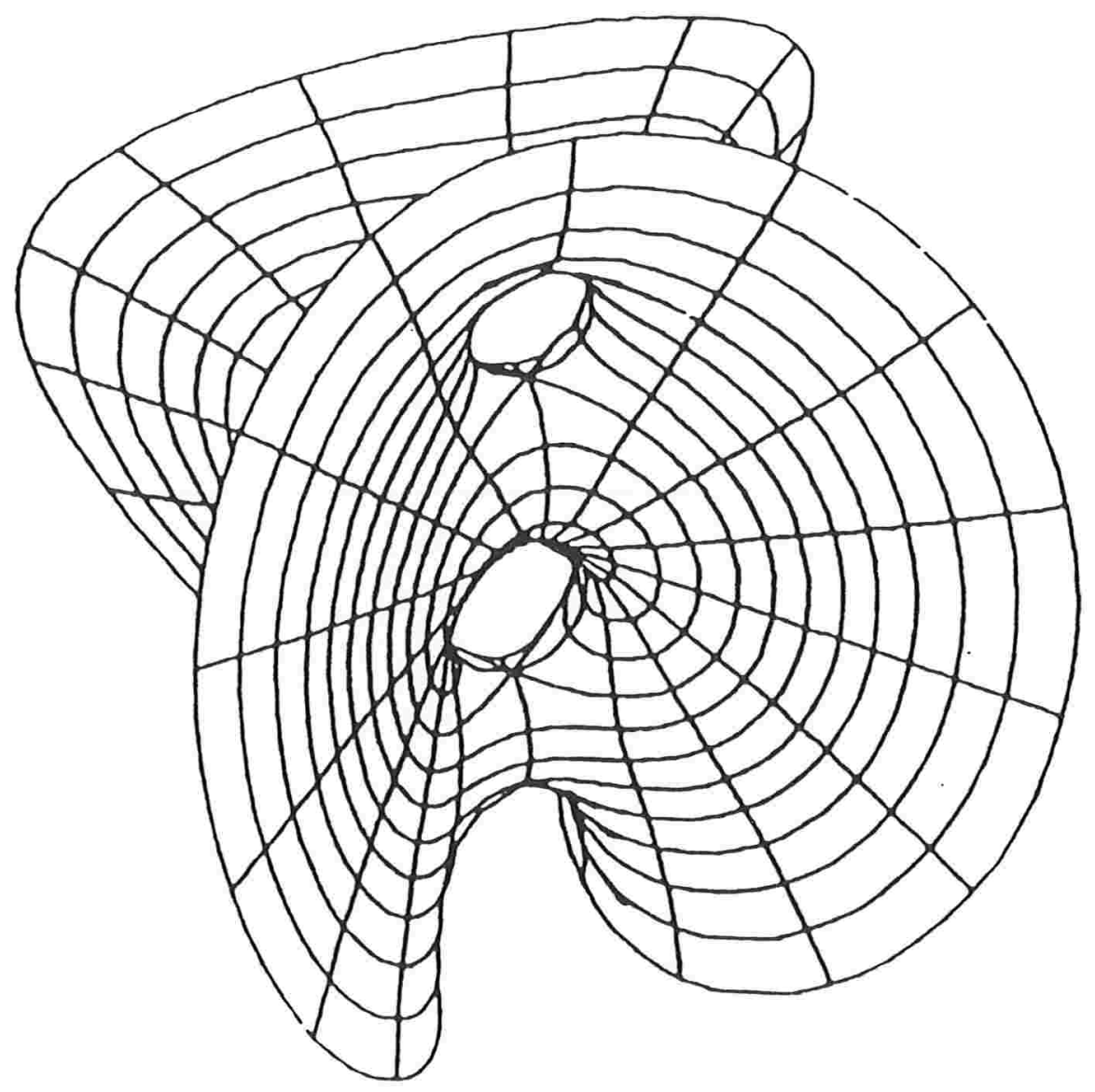




\section{Capítulo 4}

\section{Generalização da Superfície de Chen-Gackstätter de Gênero 1}

No teorema 2.2.1 do capítulo 2 foi demonstrado que existe uma superfície mínima completa do $\mathbb{R}^{3}$ com gênero 1 , curvatura total $-8 \pi$ e um único fim. Além disso, na seção 3.1 do capítulo 3 , fizemos uma adaptação à linguagem hiperelíptica para tal caso, de modo que poderíamos construí-la com:

$$
\left\{\begin{array}{l}
R=\bar{R} \backslash\{\infty\} \\
g(z)=\frac{2 A \sqrt{z\left(z^{2}-e_{1}^{2}\right)}}{z}=\frac{2 A w(z)}{z} \\
\eta(z)=\frac{z d z}{\sqrt{z\left(z^{2}-e_{1}^{2}\right)}}=\frac{z d z}{w(z)}
\end{array}\right.
$$

onde $\bar{R}$ é a superfície de Riemann de $w(z)=2 \sqrt{z\left(z-e_{1}\right)\left(z+e_{1}\right)}$.

Neste capítulo iremos generalizar a superfície descrita no teorema 2.2 .1 para uma família de imersões mínimas completas com curvatura total $-8 k \pi$, gênero $k$ e um fim de ordem $2 k+1$.

Seja $\bar{R}_{k}$ a superfície de Riemann compacta da função algébrica $w(z)$, dada por

$$
w^{k+1}(z)=z^{k}\left(z^{2}-a^{2}\right) \quad k \in \mathbb{Z}_{+}, \quad a \in \mathbb{R}_{+}
$$

Intuitivamente a superfície de Riemann $\bar{R}_{k}$ é obtida cortando a esfera de Riemann $\mathbb{C} \cup \infty$ ao longo de duas curvas ligando $\infty$ a $-a$ e 0 a $a$, e em seguida colando 
convenientemente as $k+1$ cópias destas esferas ao longo dos cortes. A superfície resultante $\bar{R}_{k}$ é então uma esfera com $k$ alças. Isto é facilmente comprovado pela equação dada no teorema 1.2 .13 , já que $\infty,-a, 0, a$ são os pontos de ramificação de ordem $k$ e o número de folhas é $n=k+1$. De 1.5, temos:

$$
4 k=2(k+1+p-1) \Rightarrow 4 k=2(k+p) \Rightarrow p=k
$$

A base para homologia de $\bar{R}_{k}$ é gerada por $2 k$ curvas fechadas não triviais de $\bar{R}_{k}$ Essas curvas serão denotadas por $\gamma_{1}^{j}, \gamma_{2}^{j}, j=1,2, \ldots, k+1$ e o comportamento delas está esquematizado na figura 4.2. A mudança de índice $j$ significa que essas curvas passam por cópias distintas das $k+1$ folhas de $\bar{R}_{k}$.

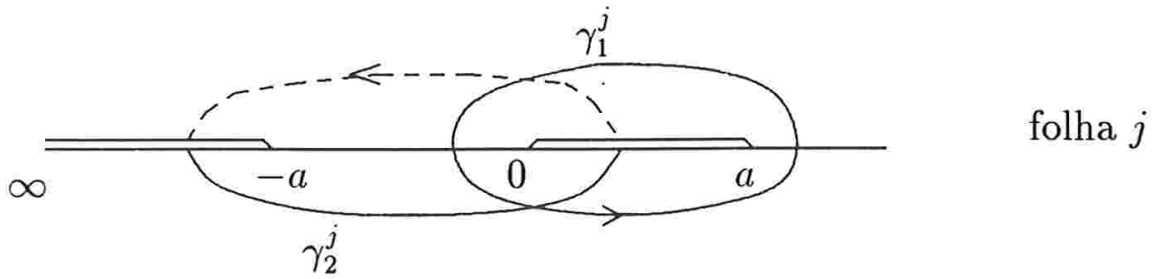

\section{Figura 4.1}

Por exemplo para $k=2$ temos uma superfície de Riemann $\bar{R}_{2}$ construída com três folhas $j=1,2,3$ e $\left\{\gamma_{1}^{1}, \gamma_{1}^{2}, \gamma_{2}^{2}, \gamma_{2}^{3}\right\}$ formam a base de sua homologia. Veja figura 4.3 abaixo.

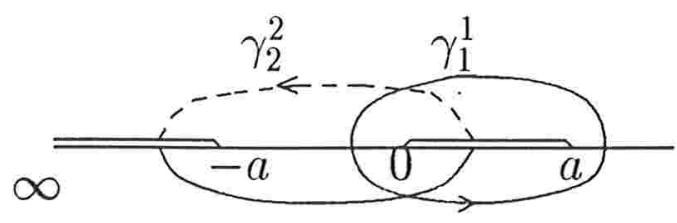

folha 1

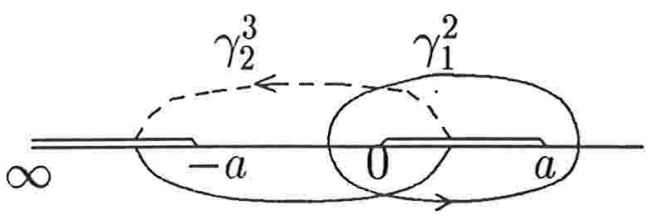

folha 2

Figura 4.2

Topologicamente: 


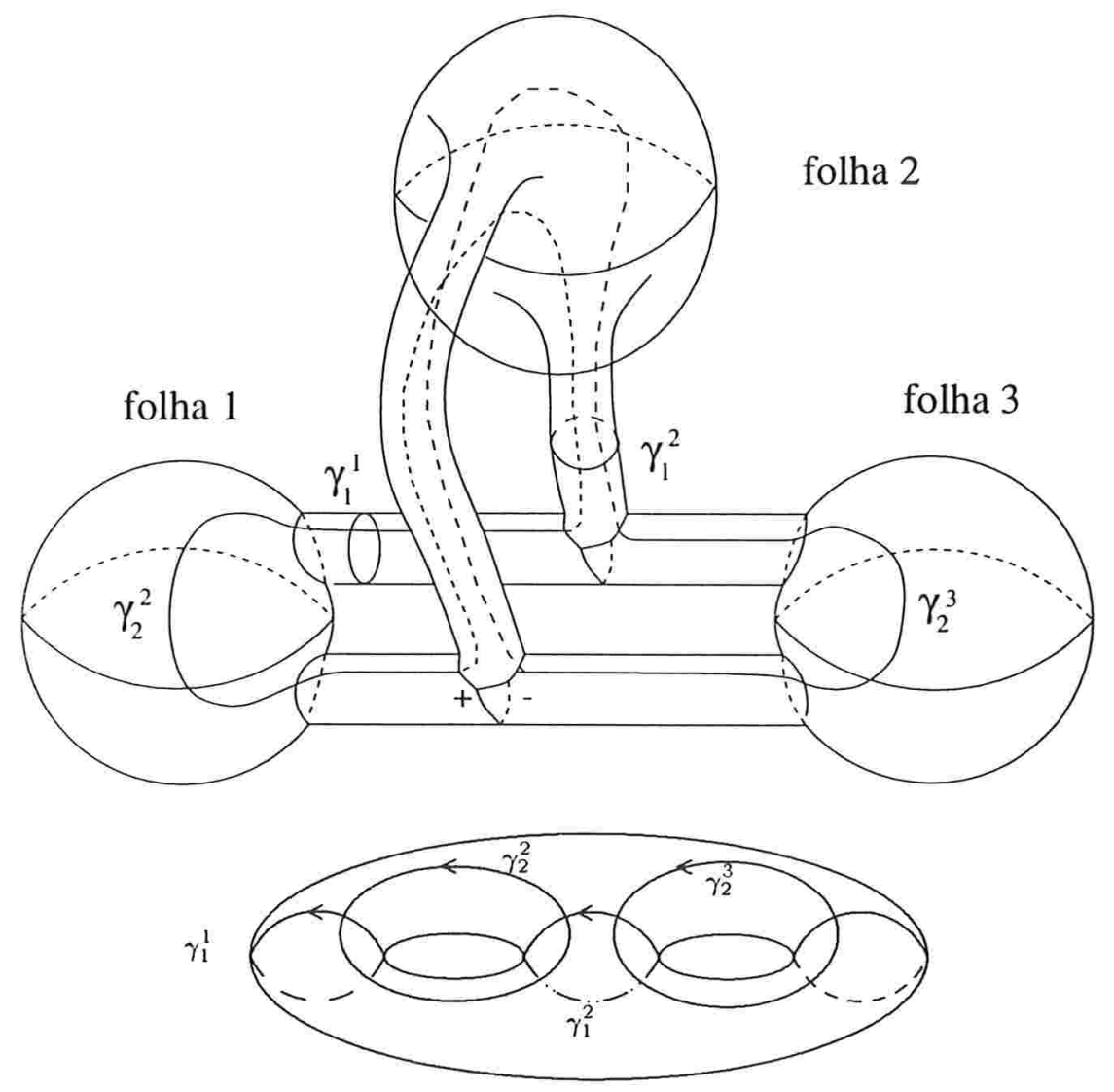

Figura 4.3

Teorema 4.0.5 (H. Wang-J.Kang 1) Existe uma superfície mínima completa $S$ do $\mathbb{R}^{3}$ com gênero $k$, curvatura total finita $C(S)=-8 k \pi$ e um único fim de ordem $2 k+1$. Esta superfície é do tipo Enneper com $k$ alças. Ela é a imersão da superfície de Riemann $\bar{R}_{k}$, da função algébrica,

$$
w(z):=\left[z^{k}\left(z^{2}-a^{2}\right)\right]^{\frac{1}{k+1}}, \quad \text { onde } \quad k \in \mathbb{Z}_{+}, \quad a \in \mathbb{R}_{+},
$$

menos um ponto de ramificação correspondente ao infinito. A representação de Weierstrass de $S$ é dada por:

$$
\begin{aligned}
g(z) & :=B \frac{w^{k}(z)}{z^{k}} \\
\eta(z) & :=\frac{z^{k}}{w^{k}(z)} d z
\end{aligned}
$$


onde $B$ é um número real a ser determinado. Em particular, quando $k=1$ temos o exemplo do teorema 2.2.1.

Para demonstrar este teorema, precisamos do lema:

Lema 4.0.6 Sobre uma superficie de Riemann compacta $\bar{R}_{k}$, consideremos as diferenciais meromorfas $o_{1}$ e $\phi_{2}$ definidas por:

$$
\begin{aligned}
\phi_{1} & :=\frac{1}{2}\left[\frac{w(z)}{z^{2}-a^{2}}-\frac{B^{2}\left(z^{2}-a^{2}\right)}{w(z)}\right] d z \\
\phi_{2} & :=\frac{i}{2}\left[\frac{w(z)}{z^{2}-a^{2}}+\frac{B^{2}\left(z^{2}-a^{2}\right)}{w(z)}\right] d z
\end{aligned}
$$

onde $\bar{R}_{k}$ é a superfície de $w(z)=\left[z^{k}\left(z^{2}-a^{2}\right)\right]^{\frac{1}{k+1}}$, com $a \in \mathbb{R}_{+} \quad e k \in \mathbb{Z}_{+}$. Então valem as seguintes igualdades:

(i) $\int_{c_{1}} \phi_{1}=\int_{c_{1}} o_{2}=0, \quad \forall z \in c_{1}: z=\varepsilon e^{i t}, t \in[0,2 \pi]$, quando $\varepsilon \rightarrow 0$

(ii) $\int_{c_{2}} \phi_{1}=\int_{c_{2}} o_{2}=0, \forall z \in c_{2}: z=a+\varepsilon e^{i t}, t \in[-\pi, \pi]$, quando $\varepsilon \rightarrow 0$

(iii) $\int_{\Gamma_{1}} \phi_{1}=\int_{\Gamma_{1}} o_{2}=0, \forall z \in \Gamma_{1}: z=\varepsilon e^{i t}, t \in[0,4 \pi]$, quando $\varepsilon \rightarrow 0$

(iv) $\int_{\Gamma_{2}} \phi_{1}=\int_{\Gamma_{2}} \phi_{2}=0, \quad \forall z \in \Gamma_{2}: z=-a+\varepsilon e^{i t}, t \in[-\pi, 3 \pi]$, quando $\varepsilon \rightarrow 0$

onde $\varepsilon$ é um real positivo suficientemente pequeno, as curvas $c_{1}$ e $c_{2}$ pertencem a uma das $j$ folhas, $\mathbb{C} \backslash\{(-\infty,-a] \cup[0, a]\}, j=1,2, \ldots, k+1$, de $w(z) ;$ o argumento de cada ramo de $w(z)$ sobre a parte superior dos cortes das folhas é $\frac{2 j \pi}{k+1} ; \Gamma_{1}$ e $\Gamma_{2}$ são curvas que passam por duas das $k+1$ folhas de $w(z)$.

Demonstração:

dem. de (i): Como $|z|=\varepsilon, c_{1}: z=\varepsilon e^{i t}, t \in[0,2 \pi]$, veja figura 4.4.

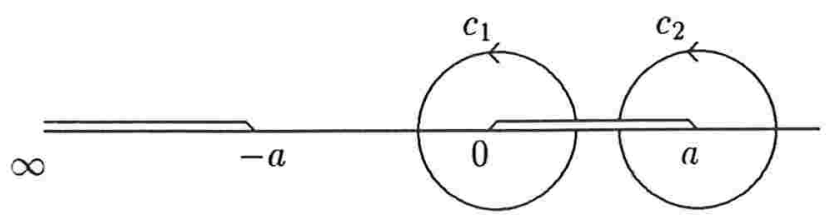




\section{Figura 4.4}

Temos,

$$
\begin{aligned}
\left|\int \phi_{1}(z)\right| \leq \frac{1}{2} \int\left|\frac{e^{\frac{2 \jmath \pi i}{k+1}}\left[z^{k}\left(z^{2}-a^{2}\right)\right]^{\frac{1}{k+1}}}{\left(z^{2}-a^{2}\right)}\right||d z|+\frac{B^{2}}{2} \int\left|\frac{\left(z^{2}-a^{2}\right)}{e^{\frac{2 \jmath \pi i}{k+1}}\left[z^{k}\left(z^{2}-a^{2}\right)\right]^{\frac{1}{k+1}}}\right||d z| \\
\left|\int \phi_{1}(z)\right| \leq \frac{1}{2} \int\left|\frac{\left[z^{k}\left(z^{2}-a^{2}\right)\right]^{\frac{1}{k+1}}}{\left(z^{2}-a^{2}\right)}\right||d z|+\frac{B^{2}}{2} \int\left|\frac{\left(z^{2}-a^{2}\right)}{\left[z^{k}\left(z^{2}-a^{2}\right)\right]^{\frac{1}{k+1}}}\right||d z| \\
\left|\int \phi_{1}(z)\right| \leq \frac{1}{2} \int\left[\frac{|z|}{\left|z^{2}-a^{2}\right|}\right]^{\frac{k}{k+1}}|d z|+\frac{B^{2}}{2} \int\left[\frac{\left|z^{2}-a^{2}\right|}{|z|}\right]^{\frac{k}{k+1}}|d z|
\end{aligned}
$$

Logo,

$$
\begin{aligned}
\left|\int_{c_{1}} \phi_{1}(z)\right| & \leq \frac{1}{2} \int_{C_{1}}\left[\frac{|z|}{a^{2}-\left|z^{2}\right|}\right]^{\frac{k}{k+1}}|d z|+\frac{B^{2}}{2} \int_{C_{1}}\left[\frac{\left|z^{2}\right|+a^{2}}{|z|}\right]^{\frac{k}{k+1}}|d z| \\
& =\frac{1}{2} \int_{0}^{2} \pi\left[\frac{\varepsilon}{a^{2}-\varepsilon^{2}}\right]^{\frac{k}{k+1}} \varepsilon d t+\frac{B^{2}}{2} \int_{0}^{2} \pi\left[\frac{\varepsilon^{2}+a^{2}}{\varepsilon}\right]^{\frac{k}{k+1}} \varepsilon d t \\
& =\frac{1}{2} \varepsilon\left[\frac{\varepsilon}{a^{2}-\varepsilon^{2}}\right]^{\frac{k}{k+1}} \int_{0}^{2 \pi} d t+\frac{B^{2}}{2} \varepsilon\left[\frac{\varepsilon^{2}+a^{2}}{\varepsilon}\right]^{\frac{k}{k+1}} \int_{0}^{2 \pi} d t \\
& =\left\{\varepsilon\left[\frac{\varepsilon}{a^{2}-\varepsilon^{2}}\right]^{\frac{k}{k+1}}+B^{2} \varepsilon^{\frac{1}{k+1}}\left[\varepsilon^{2}+a^{2}\right]^{\frac{k}{k+1}}\right\}
\end{aligned}
$$

Portanto, $\left|\int_{c_{1}} \phi_{1}\right| \rightarrow 0$, quando $\varepsilon \rightarrow 0$. De modo análogo, $\left|\int_{c_{1}} \phi_{2}\right| \rightarrow 0$, quando $\varepsilon \rightarrow 0$.

dem. de (ii): Agora em $c_{2} ;|z-a|=\varepsilon, \quad c_{2}: z=a+\varepsilon e^{i t}, \quad t \in[-\pi, \pi]$. Pela inequação 4.3 , temos

$$
\begin{aligned}
\left|\int \phi_{1}(z)\right| & \leq \frac{1}{2} \int\left[\frac{|z|}{\left|z^{2}-a^{2}\right|}\right]^{\frac{k}{k+1}}|d z|+\frac{B^{2}}{2} \int\left[\frac{\left|z^{2}-a^{2}\right|}{|z|}\right]^{\frac{k}{k+1}}|d z| \\
& =\frac{1}{2} \int\left[\frac{|z|}{|z-a||z+a|}\right]^{\frac{k}{k+1}}|d z|+\frac{B^{2}}{2} \int\left[\frac{|z+a||z-a|}{|z|}\right]^{\frac{k}{k+1}}|d z|
\end{aligned}
$$

Como, $|z+a| \geq 2 a-|z-a|,|z| \geq a-|z-a|,|z| \leq|z-a|+a$ e $|z+a| \leq|z-a|+2 a$ segue,

$$
\left|\int_{c_{2}} \phi_{1}(z)\right| \leq \frac{1}{2} \int_{0}^{2 \pi}\left[\frac{\varepsilon+a}{(2 a-\varepsilon) \varepsilon}\right]^{\frac{k}{k+1}} \varepsilon d t+\frac{B^{2}}{2} \int_{0}^{2 \pi}\left[\frac{(\varepsilon+2 a) \varepsilon}{a-\varepsilon}\right]^{\frac{k}{k+1}} \varepsilon d t
$$




$$
\left|\int_{c_{2}} \phi_{1}(z)\right| \leq\left\{\varepsilon^{\frac{1}{k+1}}\left[\frac{\varepsilon+a}{2 a-\varepsilon}\right]^{\frac{k}{k+1}}+\varepsilon B^{2}\left[\frac{\varepsilon(\varepsilon+2 a)}{a-\varepsilon}\right]^{\frac{k}{k+1}}\right\} \pi
$$

Logo, $\left|\int_{c_{2}} \phi_{1}\right| \rightarrow 0$, quando $\varepsilon \rightarrow 0$. Similarmente $\left|\int_{c_{2}} \phi_{2}\right| \rightarrow 0$, quando $\varepsilon \rightarrow 0$. dem. de (iii): $\operatorname{Em} \Gamma_{1},|z|=\varepsilon, \Gamma_{1}: z=\varepsilon e^{i t}, t \in[0,4 \pi]$, porque $\Gamma_{1}$ é uma curva que passa por duas folhas, como na figura 4.5.

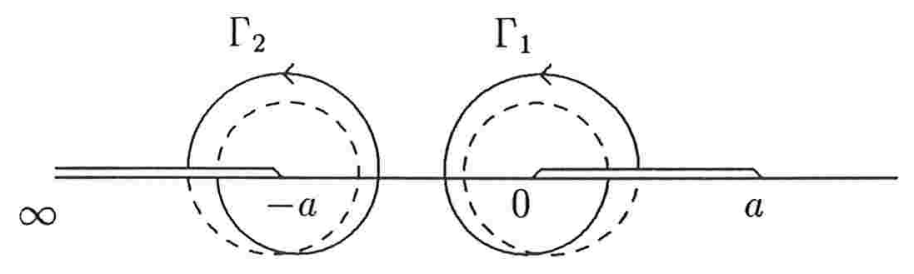

$\Gamma_{i}=\Gamma_{i}^{j} \cup \Gamma_{i}^{j-1}, i=1,2$

$\Gamma_{i}^{j}$ está na folha $j$

$\Gamma_{i}^{j-1}$ está na folha $j-1$

Figura 4.5

$$
\begin{aligned}
\int_{\Gamma_{1}} \phi_{1} & =\int_{\Gamma_{1}^{j}} \phi_{1}+\int_{\Gamma_{1}^{j-1}} \phi_{1} \\
& =\left\{\frac{1}{2} \int_{\Gamma_{1}^{j}} \frac{e^{\frac{2 j \pi i}{k+1}}\left[z^{k}\left(z^{2}-a^{2}\right)\right]^{\frac{1}{k+1}}}{\left(z^{2}-a^{2}\right)} d z+\frac{B^{2}}{2} \int_{\Gamma_{1}^{j}} \frac{\left(z^{2}-a^{2}\right)}{e^{\frac{2 j \pi i}{k+1}}\left[z^{k}\left(z^{2}-a^{2}\right)\right]^{\frac{1}{k+1}}} d z\right\}+ \\
& +\left\{\frac{1}{2} \int_{\Gamma_{1}^{j-1}} \frac{e^{\frac{2(j-1) \pi i}{k+1}}\left[z^{k}\left(z^{2}-a^{2}\right)\right]^{\frac{1}{k+1}}}{\left(z^{2}-a^{2}\right)} d z+\frac{B^{2}}{2} \int_{\Gamma_{1}^{j-1}} \frac{\left(z^{2}-a^{2}\right)}{e^{\frac{2 j \pi i}{k+1}}\left[z^{k}\left(z^{2}-a^{2}\right)\right]^{\frac{1}{k+1}}} d z\right\}
\end{aligned}
$$

Portanto,

$$
\begin{aligned}
\left|\int_{\Gamma_{1}} \phi_{1}\right| & \leq \int_{\Gamma_{1}^{j}}\left|\frac{\left[z^{k}\left(z^{2}-a^{2}\right)\right]^{\frac{1}{k+1}}}{\left(z^{2}-a^{2}\right)}\right||d z|+\frac{B^{2}}{2} \int_{\Gamma_{1}^{j}}\left|\frac{\left(z^{2}-a^{2}\right)}{\left[z^{k}\left(z^{2}-a^{2}\right)\right]^{\frac{1}{k+1}}}\right||d z|+ \\
& +\int_{\Gamma_{1}^{j-1}}\left|\frac{\left[z^{k}\left(z^{2}-a^{2}\right)\right]^{\frac{1}{k+1}}}{\left(z^{2}-a^{2}\right)}\right||d z|+\frac{B^{2}}{2} \int_{\Gamma_{1}^{j-1}}\left|\frac{\left(z^{2}-a^{2}\right)}{\left[z^{k}\left(z^{2}-a^{2}\right)\right]^{\frac{1}{k+1}}}\right||d z|+
\end{aligned}
$$

Como em $\Gamma_{1}^{j}, \quad|z|=\varepsilon$, com $z=\varepsilon e^{i t}, t \in[0,2 \pi]$ e em $\Gamma_{1}^{j-1},|z|=\varepsilon$, com $z=\varepsilon e^{i t}$, $t \in[2 \pi, 4 \pi]$ segue do ítem $(i)$ que $\left|\int_{\Gamma_{1}} \phi_{1}(z)\right| \rightarrow 0$, quando $\varepsilon \rightarrow 0$. 
dem de (iv): Com a mesma idéia de (iii); em $\Gamma_{2},|z+a|=\varepsilon$, e $z=-a+\varepsilon e^{i t}$, $t \in[-\pi, 3 \pi]$. De modo que a parte de $\Gamma_{2}$ pertencente a folha $j$ é $\Gamma_{2}^{j}:|z+a|=\varepsilon$ sendo $z=-a+\varepsilon e^{i t}, t \in[\pi, 3 \pi]$, como na figura 4.5 .

Assim,

$$
\begin{aligned}
\int_{\Gamma_{2}} \phi_{1} & =\left\{\frac{1}{2} \int_{\Gamma_{2}^{j}} \frac{e^{\frac{2 j \pi i}{k+1}}\left[z^{k}\left(z^{2}-a^{2}\right)\right]^{\frac{1}{k+1}}}{\left(z^{2}-a^{2}\right)} d z+\frac{B^{2}}{2} \int_{\Gamma_{2}^{j}} \frac{\left(z^{2}-a^{2}\right)}{e^{\frac{2 j \pi i}{k+1}}\left[z^{k}\left(z^{2}-a^{2}\right)\right]^{\frac{1}{k+1}}} d z\right\}+ \\
& +\left\{\frac{1}{2} \int_{\Gamma_{2}^{j-1}} \frac{e^{\frac{2(j-1) \pi i}{k+1}}\left[z^{k}\left(z^{2}-a^{2}\right)\right]^{\frac{1}{k+1}}}{\left(z^{2}-a^{2}\right)} d z+\frac{B^{2}}{2} \int_{\Gamma_{2}^{j-1}} \frac{\left(z^{2}-a^{2}\right)}{e^{\frac{2 j \pi i}{k+1}}\left[z^{k}\left(z^{2}-a^{2}\right)\right]^{\frac{1}{k+1}}} d z\right\}
\end{aligned}
$$

Portanto,

$$
\begin{aligned}
\left|\int_{\Gamma_{2}} \phi_{1}\right| & \leq \frac{1}{2} \int_{\Gamma_{2}^{j}}\left[\frac{|z|}{|z-a||z+a|}\right]^{\frac{k}{k+1}}|d z|+\frac{B^{2}}{2} \int_{\Gamma_{2}^{j}}\left[\frac{|z-a||z+a|}{|z|}\right]^{\frac{k}{k+1}}|d z|+ \\
& +\frac{1}{2} \int_{\Gamma_{2}^{j-1}}\left[\frac{|z|}{|z-a||z+a|}\right]^{\frac{k}{k+1}}|d z|+\frac{B^{2}}{2} \int_{\Gamma_{2}^{j-1}}\left[\frac{|z+a||z-a|}{|z|}\right]^{\frac{k}{k+1}}|d z| \\
\left|\int_{\Gamma_{2}} \phi_{1}\right| & \leq\left\{\left[\frac{\varepsilon+a}{\varepsilon(2 a-\varepsilon)}\right]^{\frac{k}{k+1}} \varepsilon+\varepsilon B^{2}\left[\frac{\varepsilon(\varepsilon+2 a)}{a-\varepsilon}\right]^{\frac{k}{k+1}}\right\} 2 \pi= \\
& =\left\{\left[\frac{\varepsilon+a}{2 a-\varepsilon}\right]^{\frac{k}{k+1}} \varepsilon^{\frac{1}{k+1}}+\varepsilon^{\frac{2 k+2}{k+1}} B^{2}\left[\frac{(\varepsilon+2 a)}{a-\varepsilon}\right]^{\frac{k}{k+1}}\right\} 2 \pi
\end{aligned}
$$

Então, $\left|\int_{\Gamma_{2}} \phi_{1}\right| \rightarrow 0$, quando $\varepsilon \rightarrow 0$. Análogamente $\left|\int_{\Gamma_{2}} \phi_{2}\right| \rightarrow 0$, quando $\varepsilon \rightarrow 0$.

Demonstração: Afirmamos que a superfície $S$, descrita no teorema 4.0.5, é obtida imergindo $R_{k}:=\bar{R}_{k} \backslash\{\infty\}$ em $\mathbb{R}^{3}$, tendo:

$$
\begin{aligned}
& g(z):=B \frac{w^{k}(z)}{z^{k}} \\
& \eta(z):=\frac{z^{k}}{w^{k}(z)} d z
\end{aligned}
$$

como representação de Weierstrass, onde $B$ uma constante real a ser determinada.

Analisaremos primeiramente o comportamento de $g$ e $\eta$, nos pontos de ramificação de $w(z): \infty,-a, 0$ e $a$. Seja $z: \bar{R}_{k} \longrightarrow \mathbb{C} \cup\{\infty\}$ um sistema de coordenadas, tal que $z\left(P_{i}\right)=e_{i}$, onde $e_{i}$ é um dos pontos de ramificação distinto do infinito. A coordenada 
local neste caso é $\zeta=\left(z-e_{i}\right)^{\frac{1}{k+1}}$. É fácil ver que das equações 4.1 e 4.4 temos:

$$
\begin{aligned}
& g(z)=\frac{B\left(z^{2}-a^{2}\right)}{\left[z^{k}\left(z^{2}-a^{2}\right)\right]^{\frac{1}{k+1}}} \\
& \eta(z)=\frac{\left[z^{k}\left(z^{2}-a^{2}\right)\right]^{\frac{1}{k+1}}}{\left(z^{2}-a^{2}\right)} d z
\end{aligned}
$$

Se $e_{i}=-a$, então $\zeta=(z+a)^{\frac{1}{k+1}}, z=\zeta^{k+1}-a$ e $d z=(k+1) \zeta^{k} d \zeta$. Logo,

$\tilde{g}(\zeta)=\frac{B \zeta^{k+1}\left(\zeta^{k+1}-2 a\right)}{\left[\left(\zeta^{k+1}-a\right)^{k}\left(\zeta^{k+1}-2 a\right) \zeta^{k+1}\right]^{\frac{1}{k+1}}}=\zeta^{k} \frac{B\left(\zeta^{k+1}-2 a\right)}{\left[\left(\zeta^{k+1}-a\right)^{k}\left(\zeta^{k+1}-2 a\right)\right]^{\frac{1}{k+1}}}$

$\tilde{\eta}(\zeta)=\frac{1}{\zeta^{k}} \frac{\left[\left(\zeta^{k+1}-a\right)^{k}\left(\zeta^{k+1}-2 a\right)\right]^{\frac{1}{k+1}}}{\zeta^{k+1}-2 a}(k+1) \zeta^{k} d \zeta=\frac{\left[\left(\zeta^{k+1}-a\right)^{k}\left(\zeta^{k+1}-2 a\right)\right]^{\frac{1}{k+1}}}{\zeta^{k+1}-2 a}(k+1) d \zeta$

Em $\zeta=0$, temos que $\tilde{g}(\zeta)$ tem um zero de ordem $k$ e $\tilde{\eta}(\zeta)$ é regular. O mesmo vale para $e_{i}=a$.

Se $e_{i}=0$, então $\zeta=z^{\frac{1}{k+1}}, z=\zeta^{k+1}$ e $d z=(k+1) \zeta^{k} d \zeta$. Logo,

$\tilde{g}(\zeta)=\frac{\left(\zeta^{k+1}-a\right)\left(\zeta^{k+1}+a\right)}{\left[\zeta^{k(k+1)}\left(\zeta^{k+1}-a\right)\left(\zeta^{k+1}+a\right)\right]^{\frac{1}{k+1}}}=\frac{1}{\zeta^{k}} \frac{\left(\zeta^{k+1}-a\right)\left(\zeta^{k+1}+a\right)}{\left[\left(\zeta^{k+1}-a\right)\left(\zeta^{k+1}+a\right)\right]^{\frac{1}{k+1}}}$

$\tilde{\eta}(\zeta)=\zeta^{k} \frac{\left[\left(\zeta^{k+1}-a\right)\left(\zeta^{k+1}+a\right)\right]^{\frac{1}{k+1}}}{\left(\zeta^{k+1}-a\right)\left(\zeta^{k+1}+a\right)}(k+1) \zeta^{k} d \zeta=\zeta^{2 k} \frac{\left[\left(\zeta^{k+1}-a\right)\left(\zeta^{k+1}+a\right)\right]^{\frac{1}{k+1}}}{\left(\zeta^{k+1}-a\right)\left(\zeta^{k+1}+a\right)}(k+1) d \zeta$

Portanto, $g$ tem um polo se ordem $k$ e $\eta$ tem um zero de ordem $2 k$ em $z=0$.

Agora, se $z\left(P_{i}\right)=\infty$, a coordenada local é $\zeta=\frac{1}{z^{\frac{1}{k+1}}}, z=\frac{1}{\zeta^{k+1}}$ e $d z=\frac{-(k+1)}{\zeta^{k+2}} d \zeta$. Logo,

$$
\begin{aligned}
\tilde{g}(\zeta) & =\frac{\left(\frac{1}{\zeta^{k+1}}-a\right)\left(\frac{1}{\zeta^{k+1}}+a\right)}{\left[\frac{1}{\zeta^{k(k+1)}}\left(\frac{1}{\zeta^{k+1}}-a\right)\left(\frac{1}{\zeta^{k+1}}+a\right)\right]^{\frac{1}{k+1}}}=\frac{\zeta^{-2(k+1)}\left(1-\zeta^{k+1} a\right)\left(1+\zeta^{k+1} a\right)}{\zeta^{-(k+2)}\left[\left(1-\zeta^{k+1} a\right)\left(1+\zeta^{k+1} a\right)\right]^{\frac{1}{k+1}}} \\
& =\frac{1}{\zeta^{k}} \frac{\left(1-\zeta^{k+1} a\right)\left(1+\zeta^{k+1} a\right)}{\left[\left(1-\zeta^{k+1} a\right)\left(1+\zeta^{k+1} a\right)\right]^{\frac{1}{k+1}}} ; \\
\tilde{\eta}(\zeta) & =\zeta^{k} \frac{\left[\left(1-\zeta^{k+1} a\right)\left(1+\zeta^{k+1} a\right)\right]^{\frac{1}{k+1}}}{\left(1-\zeta^{k+1} a\right)\left(1+\zeta^{k+1} a\right)} \cdot \frac{-(k+1)}{\zeta^{k+2}} d \zeta
\end{aligned}
$$




$$
=\frac{1}{\zeta^{2}} \frac{-(k+1)\left[\left(1-\zeta^{k+1} a\right)\left(1+\zeta^{k+1} a\right)\right]^{\frac{1}{k+1}}}{\left(1-\zeta^{k+1} a\right)\left(1+\zeta^{k+1} a\right)} d \zeta
$$

Logo em $\zeta=0 \quad \tilde{g}$ tem um polo de ordem $k$ e $\tilde{\eta}$ tem um polo de ordem 2. De onde segue que, em $z=\infty, g$ tem um polo de ordem $k$ e $\eta$ tem um polo de ordem 2. É fácil ver que se $z\left(P_{i}\right) \neq e_{i}$ e $\infty$, então $g$ e $\eta$ são regulares. Resumindo os cálculos acima numa única tabela, segue que

\begin{tabular}{|c|c|c|c|c|}
\hline$z$ & $-a$ & 0 & $a$ & $\infty$ \\
\hline$g(z)$ & $0^{k}$ & $\infty^{k}$ & $0^{k}$ & $\infty^{k}$ \\
\hline$\eta(z)$ & $*$ & $0^{2 k}$ & $*$ & $\infty^{2}$ \\
\hline $\mathrm{S}$ & $\begin{array}{c}\text { ponto } \\
\text { regular }\end{array}$ & $\begin{array}{c}\text { ponto } \\
\text { regular }\end{array}$ & $\begin{array}{c}\text { ponto } \\
\text { regular }\end{array}$ & $\begin{array}{c}\text { fim de ordem } \\
k_{1}=2 k+1\end{array}$ \\
\hline
\end{tabular}

Da tabela acima, temos que: $\eta$ é holomorfa em $\bar{R}_{k} \backslash\{\infty\}=R_{k}$, e nos pontos em que $g$ tem polo de ordem $k, \eta$ tem zero de ordem $2 k$. Portanto, a condição $\mathbf{c}_{1}$ do teorema 1.5.21 é satisfeita.

\section{Observação:}

Do corolário $1.2 .9, \operatorname{gr}[(\eta)]=2 p-2=2 k-2$. Então, $\mathcal{Z}-\mathcal{P}=2 k-2$ deveria ser $2 k-2$, o que acontece na tabela acima.

Se a superfície $S$ existir, então $S$ será conformemente equivalente a $\bar{R}_{k} \backslash\{\infty\}$, e então teremos $p=k, N=1, \operatorname{grau}(g)=2 k$ e $C(S)=-8 k \pi$.

Usando-se a fórmula de Jorge-Meeks-Gackstäter, temos

$$
-8 k \pi=2 \pi\left(2-2 k-1-k_{1}\right)=2 \pi\left(1-2 k-k_{1}\right) \Rightarrow k_{1}=2 k+1
$$

Quando $k=1, C(S)=-8 \pi$ e $k_{1}=3$, como no teorema 2.2.1.

Da equação 4.5 , obtemos facilmente

$$
\begin{aligned}
& \phi_{1}(z)=\frac{1}{2}\left[\frac{w(z)}{z^{2}-a^{2}}-B^{2} \frac{z^{2}-a^{2}}{w(z)}\right] d z \\
& \phi_{2}(z)=\frac{i}{2}\left[\frac{w(z)}{z^{2}-a^{2}}+B^{2} \frac{z^{2}-a^{2}}{w(z)}\right] d z \\
& \phi_{3}(z)=B d z
\end{aligned}
$$

Demonstração de $c_{2}$ :

Para provar esta condição do teorema 1.5.21, é suficiente mostrar que para qualquer 
uma das curvas descritas na figura 4.2,

$$
\operatorname{Re}\left\{\int_{\gamma_{i}^{j}} \phi_{1}\right\}=\operatorname{Re}\left\{\int_{\gamma_{i}^{j}} \phi_{2}\right\}=0, \quad i=1,2 \text { e } j=1,2, \ldots, k+1
$$

Já que $\int_{\gamma_{i}^{j}} \phi_{3}=0$.

Explicitamente os períodos de $\phi_{1}$ e $\phi_{2}$, são:

$$
\begin{aligned}
\int_{\gamma_{i}^{3}} \phi_{1} & =\frac{1}{2} \int_{\gamma_{i}^{j}} \frac{w(z)}{z^{2}-a^{2}} d z-\frac{B^{2}}{2} \int_{\gamma_{i}^{j}} \frac{z^{2}-a^{2}}{w(z)} d z \\
\int_{\gamma_{i}^{3}} \phi_{2} & =\frac{i}{2} \int_{\gamma_{i}^{j}} \frac{w(z)}{z^{2}-a^{2}} d z+\frac{i B^{2}}{2} \int_{\gamma_{i}^{j}} \frac{z^{2}-a^{2}}{w(z)} d z
\end{aligned}
$$

Seja $\omega(z)$ o ramo de $w(z)=\left[z^{k}\left(z^{2}-a^{2}\right)\right]^{\frac{1}{k+1}}$ definido sobre $\mathbb{C} \backslash\{(\infty,-a] \cup[0, a]\}$ (como na figura 2) com valores na $j$-folha de $\bar{R}_{k}$, escolhido da seguinte maneira, quando $\omega(z)$ estiver na parte superior do corte da $j$-ésima folha, temos $\arg (\omega(z))=$ $\frac{2 j \pi}{k+1}$. Em particular, sobre a parte superior de $[0, a]$ figura 4.2 ,

$$
\omega(z)=e^{\frac{2 \jmath \pi i}{k+1}}\left[x^{k}\left(x^{2}-a^{2}\right)\right]^{\frac{1}{k+1}}
$$

As curvas $\gamma_{1}^{j}, \gamma_{2}^{j}$ descritas na figura 4.2, são homotópicas as da figura 4.6 abaixo. $\mathrm{E}$ as integrais calculadas sobre aquelas curvas são então iguais as integrais calculadas sobre estas curvas da figura 4.6.

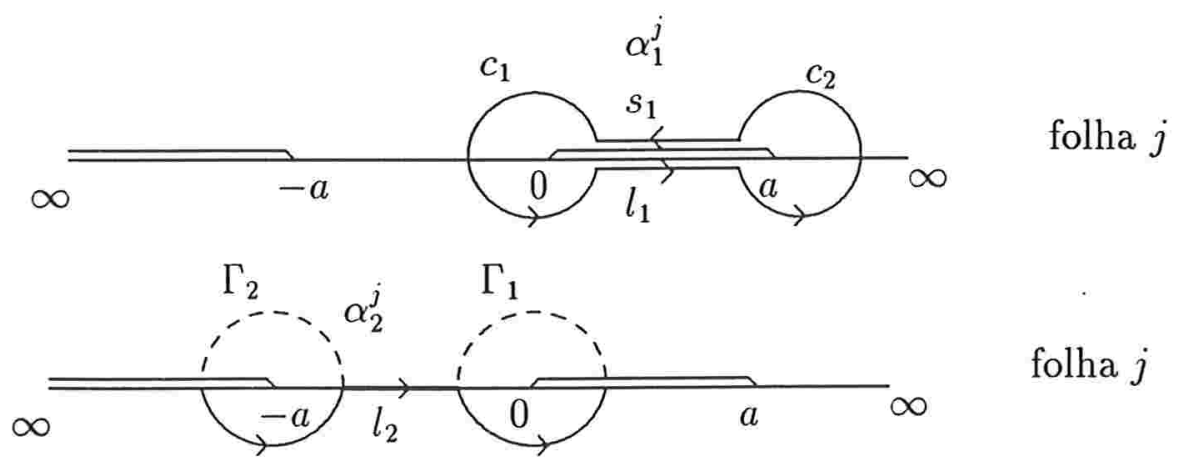

Figura 4.6 
Vamos agora calcular os períodos de $\phi_{1}$ e $\phi_{2}$.

$$
\int_{\gamma_{1}^{3}} \phi_{1}(z)=\int_{\alpha_{1}^{j}} \phi_{1}(z)=\int_{c_{1}} \phi_{1}(z) \int_{l_{1}} \phi_{1}(x)+\int_{c_{2}} \phi_{1}(z)+\int_{s_{1}} \phi_{1}(x)
$$

Pelos ítems (i) e (ii) do lema 4.0.6, temos

$$
\begin{aligned}
& \int_{\gamma_{1}^{j}} \phi_{1}(z)=\int_{l_{1}} \phi_{1}(x)+\int_{s_{1}} \phi_{1}(x) \\
& =\frac{1}{2} \int_{0}^{a} \frac{e^{\frac{2(j-1) \pi i}{k+1}}\left[x^{k}\left(x^{2}-a^{2}\right)\right]^{\frac{1}{k+1}}}{x^{2}-a^{2}} d x-\frac{B^{2}}{2} \int_{0}^{a} \frac{x^{2}-a^{2}}{e^{\frac{2(j-1) \pi i}{k+1}}\left[x^{k}\left(x^{2}-a^{2}\right)\right]^{\frac{1}{k+1}}} d x \\
& +\frac{1}{2} \int_{a}^{0} \frac{e^{\frac{2 j \pi i}{k+1}}\left[x^{k}\left(x^{2}-a^{2}\right)\right]^{\frac{1}{k+1}}}{x^{2}-a^{2}} d x-\frac{B^{2}}{2} \int_{a}^{0} \frac{x^{2}-a^{2}}{e^{\frac{2 \jmath \pi i}{k+1}}\left[x^{k}\left(x^{2}-a^{2}\right)\right]^{\frac{1}{k+1}}} d x \\
& =\frac{1}{2}\left(e^{\frac{2(\jmath-1) \pi i}{k+1}}-e^{\frac{2 \jmath \pi i}{k+1}}\right) \int_{0}^{a} \frac{\left[x^{k}\left(x^{2}-a^{2}\right)\right]^{\frac{1}{k+1}}}{x^{2}-a^{2}} d x- \\
& -\frac{B^{2}}{2}\left(e^{\frac{-2(j-1) \pi i}{k+1}}-e^{\frac{-2 j \pi i}{k+1}}\right) \int_{0}^{a} \frac{x^{2}-a^{2}}{\left[x^{k}\left(x^{2}-a^{2}\right)\right]^{\frac{1}{k+1}}} d x \\
& =\frac{1}{2}\left(e^{\frac{2(j-1) \pi i}{k+1}}-e^{\frac{2 j \pi i}{k+1}}\right) \int_{0}^{a} \frac{\left[x^{k}\left(a^{2}-x^{2}\right) e^{\pi i}\right]^{\frac{1}{k+1}}}{-\left(a^{2}-x^{2}\right)} d x- \\
& -\frac{B^{2}}{2}\left(e^{\frac{-2(j-1) \pi i}{k+1}}-e^{\frac{-2 j \pi i}{k+1}}\right) \int_{0}^{a} \frac{-\left(a^{2}-x^{2}\right)}{\left[x^{k}\left(a^{2}-x^{2}\right) e^{\pi i}\right]^{\frac{1}{k+1}}} d x \\
& =\frac{1}{2} e^{\frac{\pi i}{k+1}}\left(-e^{\frac{2(j-1) \pi i}{k+1}}+e^{\frac{2 j \pi i}{k+1}}\right) \int_{0}^{a}\left[\frac{x}{a^{2}-x^{2}}\right]^{\frac{k}{k+1}} d x- \\
& -\frac{B^{2}}{2} e^{\frac{-\pi i}{k+1}}\left(-e^{\frac{-2(\jmath-1) \pi i}{k+1}}+e^{\frac{-2 \jmath \pi i}{k+1}}\right) \int_{0}^{a}\left[\frac{a^{2}-x^{2}}{x}\right]^{\frac{k}{k+1}} d x \\
& \int_{\gamma_{1}^{\prime}} \phi_{1}(z)=\frac{1}{2} e^{\frac{2 \jmath \pi i}{k+1}}\left(e^{\frac{\pi i}{k+1}}-e^{\frac{-\pi i}{k+1}}\right) \int_{0}^{a}\left[\frac{x}{a^{2}-x^{2}}\right]^{\frac{k}{k+1}} d x- \\
& -\frac{B^{2}}{2} e^{\frac{-2 \jmath \pi i}{k+1}}\left(e^{\frac{-\pi i}{k+1}}-e^{\frac{\pi i}{k+1}}\right) \int_{0}^{a}\left[\frac{a^{2}-x^{2}}{x}\right]^{\frac{k}{k+1}} d x
\end{aligned}
$$

Pondo, $C_{j}:=e^{\frac{2 \jmath \pi i}{k+1}}\left(e^{\frac{\pi i}{k+1}}-e^{\frac{-\pi i}{k+1}}\right)$,

$$
F(a)=F:=\int_{0}^{a}\left[\frac{x}{a^{2}-x^{2}}\right]^{\frac{k}{k+1}} d x \quad \text { e } \quad G(a)=G:=\int_{0}^{a}\left[\frac{a^{2}-x^{2}}{x}\right]^{\frac{k}{k+1}} d x
$$


Temos:

$$
\int_{\gamma_{1}^{j}} \phi_{1}(z)=\frac{1}{2}\left(C_{j} F-B^{2} \overline{C_{j}} G\right)
$$

Similarmente, levando-se em consideração a equação 4.8 e os ítems (i) e (ii) do lema 4.0.6, temos.

$$
\int_{\gamma_{1}^{J}} \phi_{2}(z)=\frac{i}{2}\left(C_{j} F+B^{2} \overline{C_{j}} G\right)=\frac{1}{2}\left(i C_{j} F-B^{2}\left(\overline{i C_{j}}\right) G\right)
$$

Agora, calculemos $\int_{\gamma_{2}^{\prime}} \phi_{1}(z)$. Temos (ver figura 4.6):

$$
\int_{\gamma_{2}^{J}} \phi_{1}(z)=\int_{\alpha_{2}^{J}} \phi_{1}(z)=\int_{\Gamma_{1}} \phi_{1}(z)+\int_{l_{2}-} \phi_{1}(x)+\int_{\Gamma_{2}} \phi_{1}(z)+\int_{l_{2}} \phi_{1}(x)
$$

Segue então imediatamente dos ítems (iii) e (iv) do lema 4.0.6 que:

$$
\int_{\Gamma_{1}} \phi_{1}(z)=\int_{\Gamma_{2}} \phi_{1}(z)=0
$$

Logo,

$$
\begin{aligned}
\int_{\gamma_{2}^{3}} \phi_{1}(z) & =\int_{l_{2}^{-}} \phi_{1}(x)+\int_{l_{2}} \phi_{1}(x)= \\
& =\frac{1}{2} \int_{0}^{-a} \frac{e^{\frac{2 j \pi i}{k+1}}\left[x^{k}\left(x^{2}-a^{2}\right)\right]^{\frac{1}{k+1}}}{x^{2}-a^{2}} d x-\frac{B^{2}}{2} \int_{0}^{-a} \frac{x^{2}-a^{2}}{e^{\frac{2 j \pi i}{k+1}}\left[x^{k}\left(x^{2}-a^{2}\right)\right]^{\frac{1}{k+1}}} d x \\
& +\frac{1}{2} \int_{-a}^{0} \frac{e^{\frac{2(j-1) \pi i}{k+1}}\left[x^{k}\left(x^{2}-a^{2}\right)\right]^{\frac{1}{k+1}}}{x^{2}-a^{2}} d x-\frac{B^{2}}{2} \int_{-a}^{0} \frac{x^{2}-a^{2}}{e^{\frac{2(j-1) \pi i}{k+1}}\left[x^{k}\left(x^{2}-a^{2}\right)\right]^{\frac{1}{k+1}}} d x
\end{aligned}
$$

Fazendo a mudança de variável $x=-y$ e invertendo os limites de integração, obtemos:

$$
\begin{aligned}
\int_{\gamma_{2}^{j}} \phi_{1}(z) & =\frac{1}{2} e^{\frac{2 \jmath \pi i}{k+1}} \int_{0}^{a} \frac{\left[(-1)^{k+1} x^{k}\left(a^{2}-x^{2}\right)\right]^{\frac{1}{k+1}}}{-\left(a^{2}-x^{2}\right)}(-d x)- \\
& -\frac{B^{2}}{2} e^{\frac{-2 \jmath \pi i}{k+1}} \int_{0}^{a} \frac{-\left(a^{2}-x^{2}\right)}{\left[(-1)^{k+1} x^{k}\left(a^{2}-x^{2}\right)\right]}(-d x)- \\
& -\frac{1}{2} e^{\frac{2(\jmath-1) \pi i}{k+1}} \int_{0}^{a} \frac{\left[(-1)^{k+1} x^{k}\left(a^{2}-x^{2}\right)\right]^{\frac{1}{k+1}}}{-\left(a^{2}-x^{2}\right)}(-d x)+
\end{aligned}
$$




$$
\begin{aligned}
&+\frac{B^{2}}{2} e^{\frac{-2(\jmath-1) \pi i}{k+1}} \int_{0}^{a} \frac{-\left(a^{2}-x^{2}\right)}{\left[(-1)^{k+1} x^{k}\left(a^{2}-x^{2}\right)\right]}(-d x) \\
& \int_{\gamma_{2}^{\prime}} \phi_{1}(z)=-\frac{1}{2} e^{\frac{2 \jmath \pi i}{k+1}} \int_{0}^{a} \frac{\left[x^{k}\left(a^{2}-x^{2}\right)\right]^{\frac{1}{k+1}}}{a^{2}-x^{2}} d x+e^{\frac{2(\jmath-1) \pi i}{k+1}} \int_{0}^{a} \frac{\left[x^{k}\left(a^{2}-x^{2}\right)\right]^{\frac{1}{k+1}}}{a^{2}-x^{2}} d x \\
&+\frac{B^{2}}{2} e^{\frac{-2 \jmath \pi i}{k+1}} \int_{0}^{a} \frac{a^{2}-x^{2}}{\left[x^{k}\left(a^{2}-x^{2}\right)\right]} d x-\frac{B^{2}}{2} e^{\frac{-2(\jmath-1) \pi i}{k+1}} \int_{0}^{a} \frac{a^{2}-x^{2}}{\left[x^{k}\left(a^{2}-x^{2}\right)\right]} d x \\
&=-\frac{e^{\frac{-\pi i}{k+1}}}{2}\left(e^{\frac{(2 \jmath+1) \pi i}{k+1}}-e^{\frac{(2 \jmath-1) \pi i}{k+1}}\right) \int_{0}^{a} \frac{\left[x^{k}\left(a^{2}-x^{2}\right)\right]^{\frac{1}{k+1}}}{a^{2}-x^{2}} d x+ \\
&+\frac{B^{2}}{2} e^{\frac{\pi i}{k+1}}\left(e^{\frac{-(2 j+1) \pi i}{k+1}}-e^{\frac{-(2 j-1) \pi i}{k+1}}\right) \int_{0}^{a} \frac{a^{2}-x^{2}}{\left[x^{k}\left(a^{2}-x^{2}\right)\right]} d x \\
&=\frac{e^{\frac{-\pi i}{k+1}}}{2}\left\{e^{\frac{2 \jmath \pi i}{k+1}}\left(e^{\frac{\pi i}{k+1}}-e^{\frac{-\pi i}{k+1}}\right)\right\} \int_{0}^{a}\left[\frac{x}{a^{2}-x^{2}}\right]^{\frac{k}{k+1}} d x \\
&+\frac{B^{2}}{2} e^{\frac{\pi i}{k+1}}\left\{e^{\frac{-2 \jmath \pi i}{k+1}}\left(e^{\frac{-\pi i}{k+1}}-e^{\frac{\pi i}{k+1}}\right)\right\} \int_{0}^{a}\left[\frac{a^{2}-x^{2}}{x}\right] \\
&=-\frac{1}{2} e^{\frac{-\pi i}{k+1}} C_{j} F+\frac{B^{2}}{2} e^{\frac{\pi i}{k+1}} \overline{C_{j}} G \\
& \int_{\gamma_{2}^{\prime}} \phi_{1}(z)-\frac{1}{2}\left\{\left(e^{\frac{-\pi i}{k+1}} C_{j}\right) F-B^{2}\left(e^{\frac{-\pi i}{k+1}} C_{j}\right) G\right. \\
& \int^{k}
\end{aligned}
$$

\begin{tabular}{|c|c|c|}
\hline curvas & $\gamma_{1}^{j}$ & $\gamma_{2}^{j}$ \\
\hline $\int \phi_{1}(z)$ & $\frac{1}{2}\left(C_{j} F-B^{2} \overline{C_{j}} G\right)$ & $-\frac{1}{2}\left[\left(C_{j} e^{\frac{-\pi i}{k+1}}\right) F-B^{2}\left(C_{j} e^{\frac{-\pi i}{k+1}}\right) G\right]$ \\
\hline $\int \phi_{2}(z)$ & $\frac{1}{2}\left[\left(i C_{j}\right) F-B^{2}\left(\overline{i C_{j}}\right) G\right]$ & $-\frac{1}{2}\left[\left(i C_{j} e^{\frac{-\pi i}{k+1}}\right) F-B^{2}\left(i C_{j} e^{\frac{-\pi i}{k+1}}\right) G\right]$ \\
\hline
\end{tabular}

Além disso, pela equação 4.8 e os ítems (iii) e (iv) do lema 4.0.6 e com cálculos iguais aos acima, tem-se:

$$
\int_{\gamma_{2}^{\prime}} \phi_{2}(z)=-\frac{1}{2}\left\{\left(i e^{\frac{-\pi i}{k+1}} C_{j}\right) F-B^{2}\left(\overline{i e^{\frac{-\pi t}{k+1}} C_{j}}\right) G\right\} .
$$

O que encerra os cálculos dos períodos.

O próximo passo é provar que a parte real de cada período acima é igual a zero, para uma escolha conveniente da constante $B$.

$$
\begin{aligned}
\operatorname{Re}\left\{\int_{\gamma_{1}^{3}} \phi_{1}\right\} & =\frac{1}{2}\left\{\frac{\left(C_{j} F-B^{2} \overline{C_{j}} G\right)+\left(\overline{C_{j} F-B^{2} \overline{C_{j}} G}\right)}{2}\right\} \\
& =\frac{1}{4}\left\{\left(C_{j}+\overline{C_{j}}\right)\left(F-B^{2} G\right)\right\}=0 \Leftrightarrow C_{j}+\overline{C_{j}}=0 \text { ou } F-B^{2} G=0
\end{aligned}
$$


Agora se $C_{j}+\overline{C_{j}}=0$, então $C_{j}$ é puramente imaginário, logo $\int_{\gamma_{1}^{\prime}} \phi_{1}$ também o é. Por outro lado $F-B^{2} G=0 \Leftrightarrow B={ }_{-}^{+} \sqrt{F / G}$. A constante $B=-\sqrt{F / G}$ nos dá apenas uma superfície refletida da obtida considerando $B=\sqrt{F / G}$. Resta verificar que este valor para $B$ é o mesmo para todos os períodos e que esse valor realmente existe. De fato,

$$
\begin{aligned}
\operatorname{Re}\left\{\int_{\gamma_{2}^{\prime}} \phi_{1}\right\} & =-\frac{1}{4}\left\{\left(C_{j} e^{\frac{-\pi i}{k+1}}\right) F-B^{2}\left(\overline{C_{j} e^{\frac{-\pi i}{k+1}}}\right) G+\left(\overline{C_{j} e^{\frac{-\pi i}{k+1}}}\right) F-B^{2}\left(C_{j} e^{\frac{-\pi i}{k+1}}\right) G\right\} \\
& =\frac{1}{4}\left\{\left[\left(C_{j} e^{\frac{-\pi i}{k+1}}\right)+\left(\overline{C_{j} e^{\frac{-\pi i}{k+1}}}\right)\right]\left(F-B^{2} G\right)\right\}
\end{aligned}
$$

Logo,

$$
\operatorname{Re}\left\{\int_{\gamma_{2}^{\prime}} \rho_{1}\right\}=0, \quad \text { se } B=\sqrt{F / G} \text { ou }\left(C_{j} e^{\frac{-\pi i}{k+1}}\right)+\left(\overline{C_{j} e^{\frac{-\pi i}{k+1}}}\right)=0 .
$$

Se $\left(C_{j} e^{\frac{-\pi i}{k+1}}\right)+\left(\overline{C_{j} e^{\frac{-\pi i}{k+1}}}\right)=0$, então o complexo $\alpha=C_{j} e^{\frac{-\pi i}{k+1}}$ é puramente imaginário, e

$$
\int_{\gamma_{2}^{\prime}} \phi_{1}=-\frac{1}{2}\left(\alpha F-B^{2} \bar{\alpha} G\right)=-\frac{1}{2}\left(\alpha F-B^{2} \alpha G\right)=-\frac{1}{2} \alpha\left(F+B^{2} G\right) \in \mathbb{C}
$$

Portanto, $\operatorname{Re}\left\{\int_{\gamma_{2}^{\prime}} \phi_{1}\right\}=0$.

Analogamente,

$$
\begin{aligned}
\operatorname{Re}\left\{\int_{\gamma_{1}^{\prime}} \phi_{2}\right\} & =\frac{1}{4}\left\{\left[\left(i C_{j}+\left(\overline{C_{j}}\right)\right]\left(F-B^{2} G\right)\right\}\right. \\
\left\{\int_{\gamma_{2}^{\prime}} \phi_{2}\right\} & =-\frac{1}{4}\left\{\left[\left(i C_{j} e^{\frac{-\pi i}{k+1}}\right)+\left(\overline{i C_{j} e^{\frac{-\pi i}{k+1}}}\right)\right]\left(F-B^{2} G\right)\right\}
\end{aligned}
$$

Portanto, $\operatorname{Re}\left\{\int_{\gamma_{1}^{\prime}} \phi_{2}\right\}=\operatorname{Re}\left\{\int_{\gamma_{2}^{\prime}} \phi_{2}\right\}=0$, se $B=\sqrt{F / G}$. Observe que não podemos ter: $\quad C_{j}+\overline{C_{j}}=0, \quad\left(i C_{j}\right)+\left(\overline{i C_{j}}\right)=0, \quad\left(C_{j} e^{\frac{-\pi i}{k+1}}\right)+\left(\overline{C_{j} e^{\frac{-\pi i}{k+1}}}\right)=0 \quad \mathrm{e}$ $\left(i C_{j} e^{\frac{-\pi i}{k+1}}\right)+\left(\overline{i C_{j} e^{\frac{-\pi i}{k+1}}}\right)=0$, válidos simultaneamente. Por exemplo, se $C_{j}+\overline{C_{j}}=0$, temos que $\operatorname{Re}\left\{C_{j}\right\}=0$; e se $\left(i C_{j}\right)+\overline{\left(i C_{j}\right)}=0$, temos que $\operatorname{Re}\left\{i C_{j}\right\}=-\operatorname{Im}\left\{C_{j}\right\}=0$. Portanto, se as duas condições são satisfeitas $C_{j}=0, \forall j$, o que é uma contradição. Logo, devemos ter $F-B^{2} G=0$. 
Por último provaremos que, $F>0$ e $G>0$ e são finitos. Seja $0 \leq x \leq a$ e $0<a<\infty$, então é fácil ver que:

$$
\frac{x}{2 a^{2}} \leq \frac{x}{a^{2}-x^{2}} \leq \frac{1}{a-x} \quad \text { e } \quad a-x \leq \frac{a^{2}-x^{2}}{x} \leq \frac{2 a^{2}}{x}
$$

Logo, integrando temos:

$$
\begin{aligned}
& 0<\frac{k+1}{(2 k+1) 2^{\frac{k}{k+1}}} a^{\frac{1}{k+1}} \leq F \leq(k+1) a^{\frac{1}{k+1}} \quad \text { e } \\
& 0<\frac{k+1}{(2 k+1)} a^{\frac{2 k+1}{k+1}} \leq G \leq(k+1) 2^{\frac{k}{k+1}} a^{\frac{2 k+1}{k+1}}
\end{aligned}
$$

$\mathrm{O}$ que prova $\mathrm{c}_{2}$.

\section{Demonstração de $c_{3}$}

Usando coordenadas locais $\zeta=\frac{1}{z^{\frac{1}{k+1}}}$, temos $z=\frac{1}{\zeta^{k+1}}$ e $d z=-\frac{(k+1)}{\zeta^{k+2}} d \zeta$ e as equações 4.6 e 4.7 podemos escrever,

$$
\begin{aligned}
& \tilde{\phi}_{1}(\zeta)=\phi_{1}\left(\frac{1}{\zeta^{k+1}}\right)=\frac{1}{\zeta^{2 k+2}} h(\zeta) \\
& \tilde{\phi}_{2}(\zeta)=\frac{i}{\zeta^{2 k+2}} \tilde{h}(\zeta)
\end{aligned}
$$

onde $h$ e $\tilde{h}$ são funções analíticas numa vizinhança de $\zeta=0$ e $h(0) \neq 0$ e $\tilde{h}(0) \neq 0$. Portanto em $\zeta=0, \tilde{\phi}_{1} \tilde{\phi}_{2}$ tem um polo de ordem $2 k+2$, assim $\phi_{1}$ e $\phi_{2}$ têm um polo de ordem $2 k+2$ em $z=\infty$ que é o fim da superfície. Disto concluímos que,

$$
\operatorname{Res}\left(\phi_{1} ; \infty\right)=\operatorname{Res}\left(\phi_{2} ; \infty\right)=\operatorname{Res}\left(\phi_{3} ; \infty\right)=0 .
$$

\section{Demonstração de $\mathbf{c}_{4}$}

Já vimos na demonstração $\mathbf{c}_{\boldsymbol{4}}$ do teorema 3.2 .1 , que $\left|\tilde{\phi}_{2}(\zeta)\right| \leq \tilde{\lambda}(\zeta)|d \zeta|$, logo da equação 4.9 , segue que a superfície também é completa. 
Quando $k=2$ no teorema 4.0.5, conforme as figuras 4.2 e 4.3 , o gráfico da superfície mínima em $\mathbb{R}^{3}$ de gênero 2 , curvatura total $-16 \pi$ e um único fim do tipo Enneper é dada em [21] como na figura abaixo.

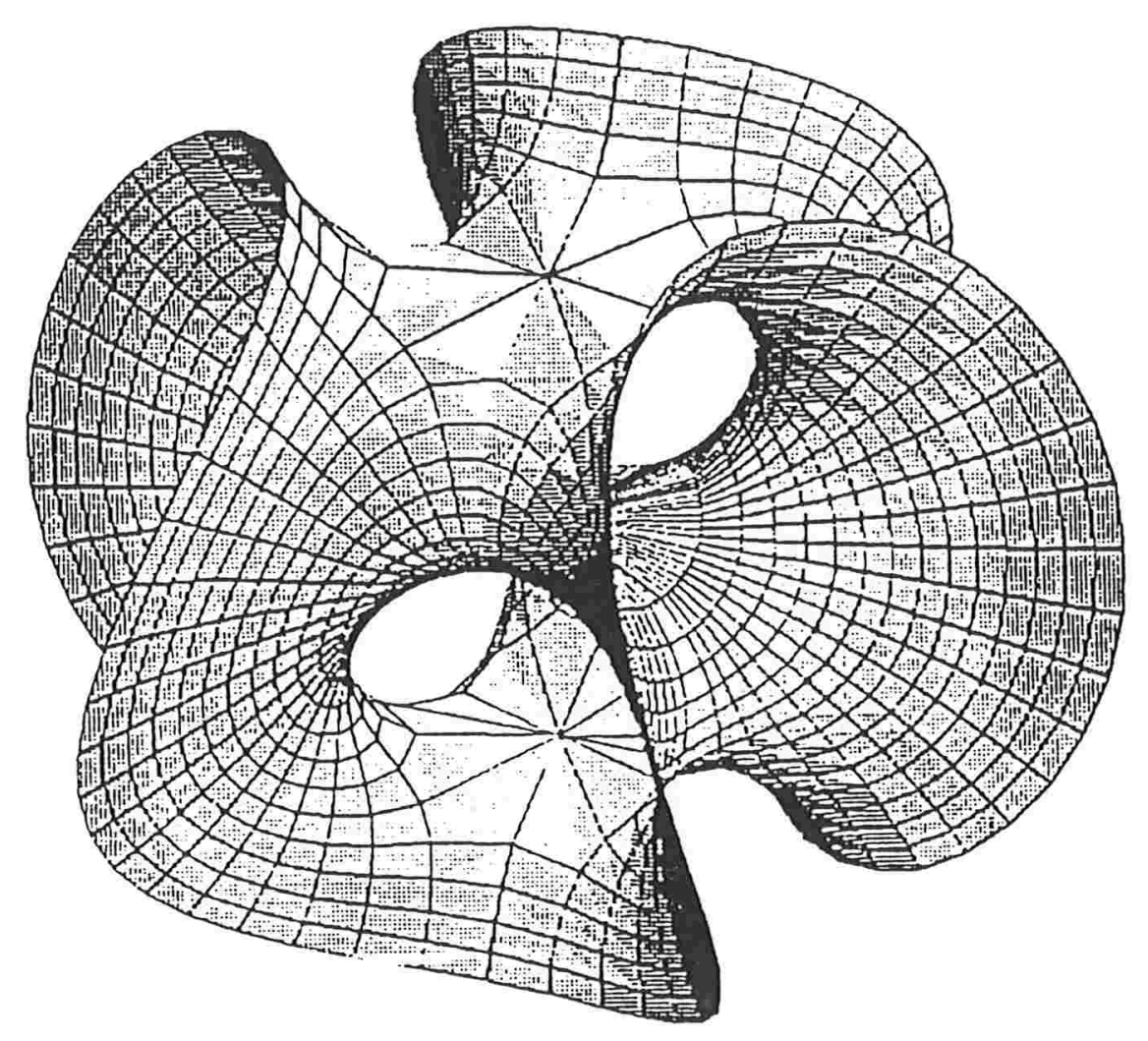




\section{Capítulo 5}

\section{Generalização da Superfície de Chen-Gackstätter de Gênero 2}

Neste capítulo o teorema principal generaliza a superfície de gênero 2 e curvatura total $-12 \pi$ descrita no teorema 3.2 .1 do capítulo 3 para uma família de imersões mínimas completas com curvatura total $-12 k \pi$, gênero $2 k$ e um fim de ordem $2 k+1$.

Seja $\bar{R}_{2 k}$ a superfície de Riemann compacta da função algébrica $w(z)$, dada por

$$
w(z)^{k+1}=z\left(z^{2}-a^{2}\right)^{k}\left(z^{2}-b^{2}\right), k \in \mathbb{Z}_{+},
$$

onde $a$ e $b$ são constantes reais satisfazendo, $0<a<b$ e $k$ é um inteiro positivo maior ou igual a 1 . Um modo topológico de construir a superfície $\bar{R}_{2 k}$, é colar de modo conveniente as $k+1$ cópias de $\mathbb{C} \cup\{\infty\}$ ao longo dos cortes feitos ligando $-b$ à $-a \quad 0$ à $a$ e $b$ à $\infty$, como foi feito na figura 1.9. A superfície resultante, $\bar{R}_{2 k}$, é homeomorfa a esfera com $2 k$ alças, isto é, tem gênero $2 k$. De fato, pela equação de Riemann, do teorema 1.2.13, $V=2(n+p-1)$. Como os pontos de ramificação $-b,-a, 0, a, b$ e $\infty$ são todos de ordem $k$ e o número de folhas usadas na construção de $\bar{R}_{2 k}$ é $n=k+1$ então,

$$
6 k=2(k+1+p-1)=2(k+p) \Rightarrow p=2 k
$$

A base para a homologia de $\bar{R}_{2 k}$ é formada por $4 k$ curvas fechadas não triviais sobre 
$\bar{R}_{2 k}$. Essas curvas serão denotadas por $\gamma_{1}^{j}, \gamma_{2}^{j}, \gamma_{3}^{j}$ e $\gamma_{4}^{j}, j=1,2, \ldots, k+1$, indicadas na figura 5.1 abaixo. A mudança de índice $j$, nos diz que as curvas acima estão em cópias diferentes dentre as $k+1$ folhas de $w(z)$. Observemos que quando $k=1$ essas curvas são as mesmas da figura 3.6 do teorema 3.2.1 do capítulo 3 .

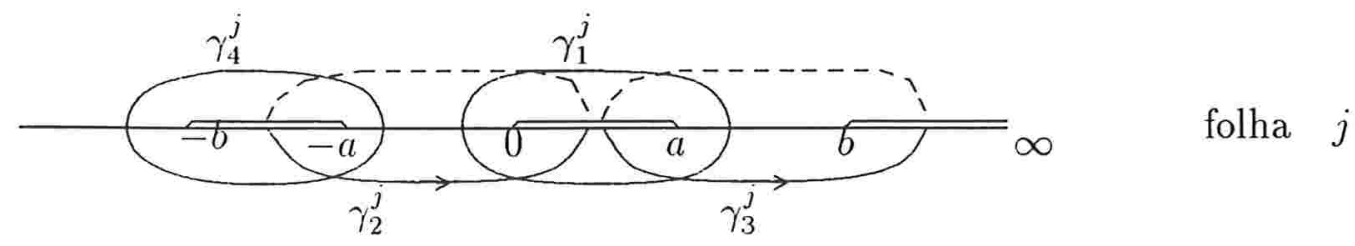

\section{Figura 5.1}

Por exemplo para $k=2$, temos três folhas do tipo $\mathbb{C} \backslash\{[-b,-a] \cup[0, a] \cup[b, \infty)\}$, e oito curvas formando a base para homologia de $\bar{R}_{2 k}$, indicadas nas figuras abaixo.
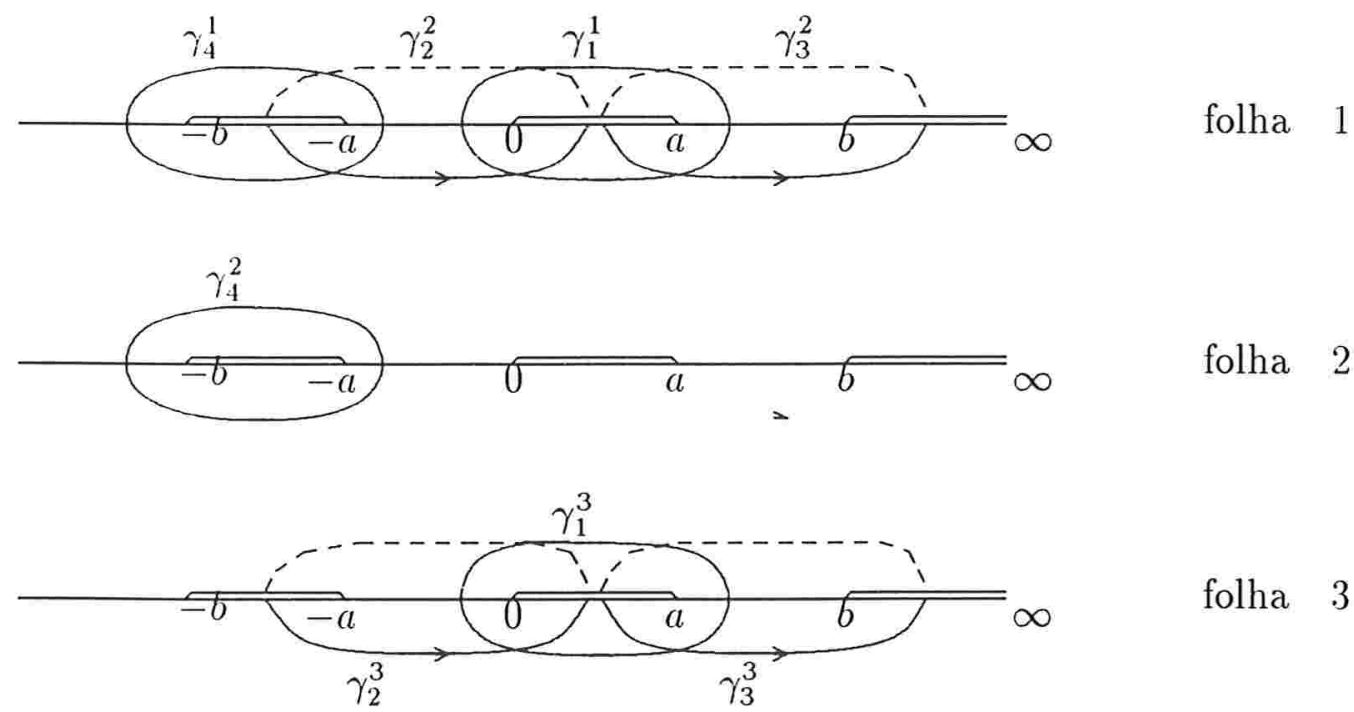

Figura 5.2 

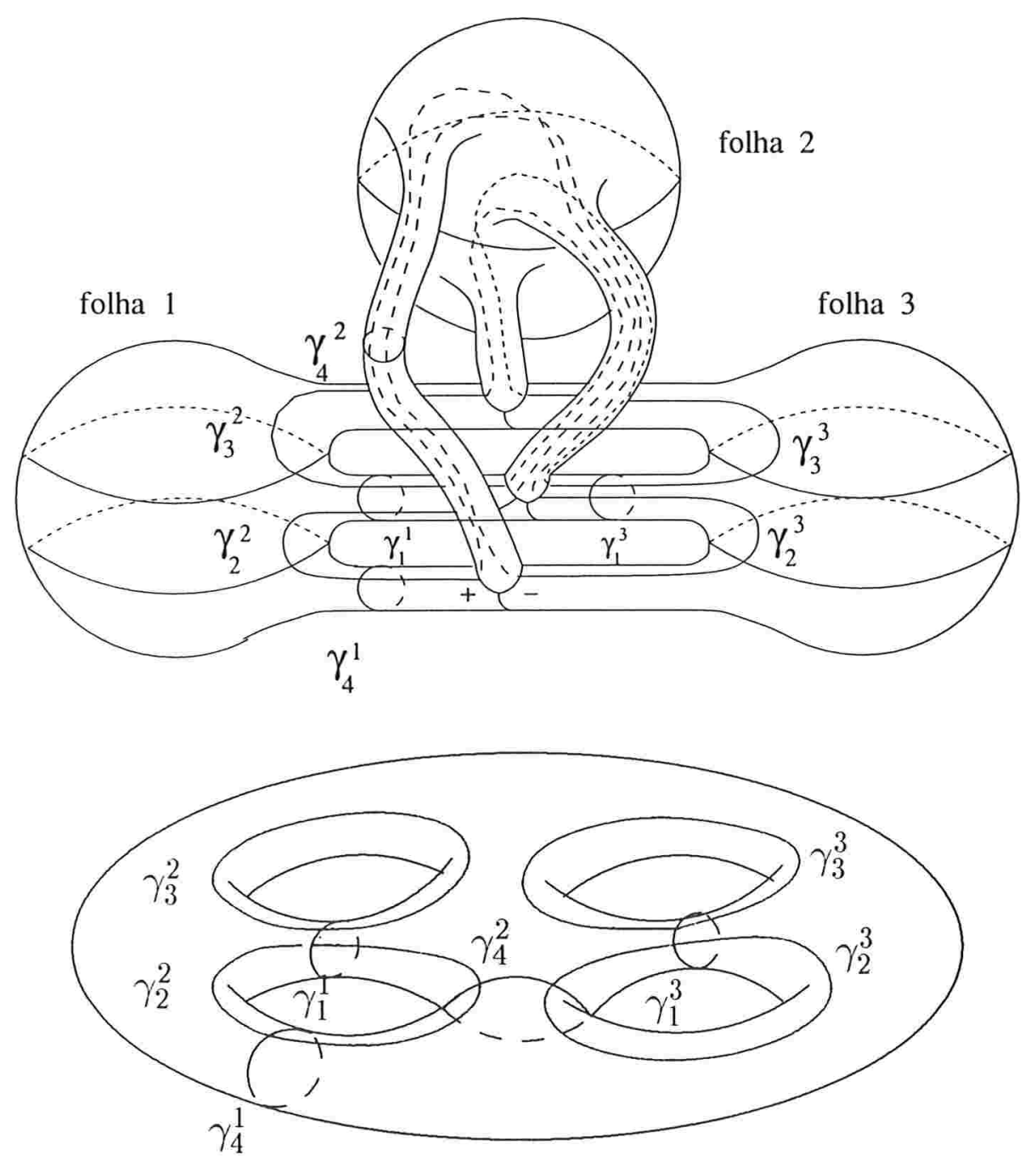

\section{Figura 5.3}

Teorema 5.0.7 (H. Wang-J.Kang 2) Existe uma superfície mínima completa $S$ do $\mathbb{R}^{3}$ com gênero $2 k$, curvatura total finita $C(S)=-12 k \pi$ e um único fim de ordem $2 k+1$. Esta superfície é do tipo Enneper com $2 k$ alças. Ela é a imersão da superfície de Riemann $\bar{R}_{2 k}$, da função algébrica,

$$
w(z):=\left[z\left(z^{2}-a^{2}\right)^{k}\left(z^{2}-b^{2}\right)\right]^{\frac{1}{k+1}}, \quad \text { onde } \quad k \in \mathbb{Z}_{+}, \quad 0<a<b, \quad a, b \in \mathbb{R},
$$


menos um ponto de ramificação correspondente ao infinito. A representação de Weierstrass de $S$ é dada por:

$$
\left\{\begin{array}{l}
g(z):=\frac{B w^{k}(z)}{\left(z^{2}-a^{2}\right)^{k}} \\
\eta(z):=\frac{\left(z^{2}-a^{2}\right)^{k}}{w^{k}(z)} d z
\end{array}\right.
$$

onde $B$ é um número real a ser determinado. Em particular, quando $k=1$ temos $o$ exemplo do teorema 3.2.1.

\section{Demonstração:}

O par $(g, \eta)$, dado em 5.2 , candidato a representação de Weierstrass da superfície $S$, é dado explicitamente por:

$$
\begin{aligned}
g(z) & =\frac{B z\left(z^{2}-b^{2}\right)}{\left[z\left(z^{2}-a^{2}\right)^{k}\left(z^{2}-b^{2}\right)\right]^{\frac{1}{k+1}}} \\
\eta(z) & =\frac{\left[z\left(z^{2}-a^{2}\right)^{k}\left(z^{2}-b^{2}\right)\right]^{\frac{1}{k+1}}}{z\left(z^{2}-b^{2}\right)} d z
\end{aligned}
$$

Logo, substituindo 5.3 nas expressões de $\phi_{1}, \phi_{2}$ e $\phi_{3}$ segue-se,

$$
\begin{aligned}
& \phi_{1}(z)=\frac{1}{2}\left\{\frac{\left[z\left(z^{2}-a^{2}\right)^{k}\left(z^{2}-b^{2}\right)\right]^{\frac{1}{k+1}}}{z\left(z^{2}-b^{2}\right)}-B^{2} \frac{z\left(z^{2}-b^{2}\right)}{\left[z\left(z^{2}-a^{2}\right)^{k}\left(z^{2}-b^{2}\right)\right]^{\frac{1}{k+1}}}\right\} d z \\
& \phi_{2}(z)=\frac{i}{2}\left\{\frac{\left[z\left(z^{2}-a^{2}\right)^{k}\left(z^{2}-b^{2}\right)\right]^{\frac{1}{k+1}}}{z\left(z^{2}-b^{2}\right)}+B^{2} \frac{z\left(z^{2}-b^{2}\right)}{\left[z\left(z^{2}-a^{2}\right)^{k}\left(z^{2}-b^{2}\right)\right]^{\frac{1}{k+1}}}\right\} d z \\
& \phi_{3}(z)=B d z
\end{aligned}
$$

Agora, examinaremos o comportamento do par $(g, \eta)$, em seus zeros e polos. Seja $z: \bar{R}_{2 k} \longrightarrow \mathbb{C} \cup \infty$ tal que $z\left(P_{i}\right)=e_{i}$, onde $e_{i}$ é um ponto de ramificação de $w(z)$ distinto do $\infty$, isto é, $-b,-a, 0, a$ e $b$. Para estes pontos consideremos a coordenada local $\zeta=\left[z-e_{i}\right]^{\frac{1}{k+1}}$. Então, $z=\zeta^{k+1}+e_{i}$ e $d z=(k+1) \zeta^{k} d \zeta$. 
Se $e_{i}=-b$ temos,

$$
\begin{aligned}
\tilde{g}(\zeta) & =\frac{B\left(\zeta^{k+1}-b\right)\left(\zeta^{k+1}-2 b\right) \zeta^{k+1}}{\left[\left(\zeta^{k+1}-b\right)\left(\left(\zeta^{k+1}-b\right)^{2}-a^{2}\right)^{k}\left(\zeta^{k+1}-2 b\right) \zeta^{k+1}\right]^{\frac{1}{k+1}}} \\
& =\zeta^{k} \frac{B\left(\zeta^{k+1}-b\right)\left(\zeta^{k+1}-2 b\right)}{\left[\left(\zeta^{k+1}-b\right)\left(\left(\zeta^{k+1}-b\right)^{2}-a^{2}\right)^{k}\left(\zeta^{k+1}-2 b\right)\right]^{\frac{1}{k+1}}}
\end{aligned}
$$

Assim,

$$
\begin{aligned}
\tilde{\eta}(\zeta) & =\frac{1}{\zeta^{k}} \frac{\left[\left(\zeta^{k+1}-b\right)\left(\left(\zeta^{k+1}-b\right)^{2}-a^{2}\right)^{k}\left(\zeta^{k+1}-2 b\right)\right]^{\frac{1}{k+1}}}{\left(\zeta^{k+1}-b\right)\left(\zeta^{k+1}-2 b\right)}(k+1) \zeta^{k} d \zeta \\
& =\frac{(k+1)\left[\left(\zeta^{k+1}-b\right)\left(\left(\zeta^{k+1}-b\right)^{2}-a^{2}\right)^{k}\left(\zeta^{k+1}-2 b\right)\right]^{\frac{1}{k+1}}}{\left(\zeta^{k+1}-b\right)\left(\zeta^{k+1}-2 b\right)} d \zeta,
\end{aligned}
$$

logo, em $\zeta=0, \quad \tilde{g}$ e $\tilde{\eta}$ têm, respectivamente, um zero de ordem $k$ e um ponto regular, o mesmo valendo para $g$ e $\eta$ em $z=-b$. O mesmo ocorre quando $z=b$.

Se $\epsilon_{i}=-a$, então $z=\zeta^{k+1}-a$ e $d z=(k+1) \zeta^{k} d \zeta$. Assim:

$$
\begin{aligned}
\tilde{g}(\zeta) & =\frac{B\left(\zeta^{k+1}-a\right)\left(\left(\zeta^{k+1}-a\right)^{2}-b^{2}\right)}{\left[\left(\zeta^{k+1}-a\right)\left(\zeta^{k+1}-2 a\right)^{k} \zeta^{k(k+1)}\left(\left(\zeta^{k+1}-a\right)^{2}-b^{2}\right)\right]^{\frac{1}{k+1}}} \\
& =\frac{1}{\zeta^{k}} \frac{B\left(\zeta^{k+1}-a\right)\left(\left(\zeta^{k+1}-a\right)^{2}-b^{2}\right)}{\left[\left(\zeta^{k+1}-a\right)\left(\zeta^{k+1}-2 a\right)^{k}\left(\left(\zeta^{k+1}-a\right)^{2}-b^{2}\right)\right]^{\frac{1}{k+1}}} \\
\tilde{\eta}(\zeta) & =\frac{\left[\left(\zeta^{k+1}-a\right)\left(\zeta^{k+1}-2 a\right)^{k} \zeta^{k(k+1)}\left(\left(\zeta^{k+1}-a\right)^{2}-b^{2}\right)\right]^{\frac{1}{k+1}}}{\left(\zeta^{k+1}-a\right)\left(\left(\zeta^{k+1}-a\right)^{2}-b^{2}\right)}(k+1) \zeta^{k} d \zeta \\
& =\zeta^{-2 k} \frac{(k+1)\left[\left(\zeta^{k+1}-a\right)\left(\zeta^{k+1}-2 a\right)^{k}\left(\left(\zeta^{k+1}-a\right)^{2}-b^{2}\right)\right]^{\frac{1}{k+1}}}{\left(\zeta^{k+1}-a\right)\left(\left(\zeta^{k+1}-a\right)^{2}-b^{2}\right)} d \zeta
\end{aligned}
$$

Portanto, $\grave{g}$ e $\tilde{\eta}$ têm em $\zeta=0$, respectivamente, um polo de ordem $k$ e um zero de ordem $2 k$. Logo em $z=-a, g$ tem um polo de ordem $k$ e $\eta$ tem um zero de ordem $2 k$. A mesma conclusão se pode tirar quando $z=a$.

Por último se $e_{i}=0$ temos,

$$
\begin{aligned}
\tilde{g}(\zeta) & =\frac{B \zeta^{k+1}\left(\zeta^{2(k+1)}-b^{2}\right)}{\left[\zeta^{k+1}\left(\zeta^{2(k+1)}-a^{2}\right)^{k}\left(\zeta^{2(k+1)}-b^{2}\right)\right]^{\frac{1}{k+1}}} \\
& =\zeta^{k} \frac{B\left(\zeta^{2(k+1)}-b^{2}\right)}{\left[\left(\zeta^{2(k+1)}-a^{2}\right)^{k}\left(\zeta^{2(k+1)}-b^{2}\right)\right]^{\frac{1}{k+1}}}
\end{aligned}
$$




$$
\begin{aligned}
\tilde{\eta}(\zeta) & =\frac{\zeta\left[\left(\zeta^{2(k+1)}-a^{2}\right)^{k}\left(\zeta^{2(k+1)}-b^{2}\right)\right]^{\frac{1}{k+1}}}{\zeta^{k+1}\left(\zeta^{2(k+1)}-b^{2}\right)}(k+1) \zeta^{k} d \zeta \\
& =\frac{(k+1)\left[\left(\zeta^{2(k+1)}-a^{2}\right)^{k}\left(\zeta^{2(k+1)}-b^{2}\right)\right]^{\frac{1}{k+1}}}{\left(\zeta^{2(k+1)}-b^{2}\right)} d \zeta
\end{aligned}
$$

Então em $z=0, g$ e $\eta$ têm, respectivamente, um zero de ordem $k$ e um ponto regular.

Finalmente no último ponto de ramificação que falta, $z\left(P_{i}\right)=\infty$, a coordenada local a ser considerada é $\zeta=\frac{1}{z^{\frac{1}{k+1}}}$, então $z=\frac{1}{\zeta^{k+1}}$ e $d z=\frac{-(k+1) d \zeta}{\zeta^{k+1}}$. Assim,

$$
\begin{aligned}
& \tilde{g}(\zeta)=\frac{B \zeta^{-(k+1)}\left(\zeta^{-2(k+1)}-b^{2}\right)}{\left[\zeta^{-(k+1)}\left(\zeta^{-2(k+1)}-a^{2}\right)^{k}\left(\zeta^{-2(k+1)}-b^{2}\right)\right]^{\frac{1}{k+1}}} \\
& =\frac{B \zeta^{-(k+1)} \zeta^{-2(k+1)}\left(1-\zeta^{2(k+1)} b^{2}\right)}{\left[\zeta^{-(k+1)} \zeta^{-2 k(k+1)}\left(1-\zeta^{2(k+1)} a^{2}\right)^{k} \zeta^{-2(k+1)}\left(1-\zeta^{2(k+1)} b^{2}\right)\right]^{\frac{1}{k+1}}} \\
& =\frac{B \zeta^{-3(k+1)}\left(1-\zeta^{2(k+1)} b^{2}\right)}{\zeta^{-(2 k+3)}\left[\left(1-\zeta^{2(k+1)} a^{2}\right)^{k}\left(1-\zeta^{2(k+1)} b^{2}\right)\right]^{\frac{1}{k+1}}} \\
& =\frac{1}{\zeta^{k}} \frac{B\left(1-\zeta^{2(k+1)} b^{2}\right)}{\left[\left(1-\zeta^{2(k+1)} a^{2}\right)^{k}\left(1-\zeta^{2(k+1)} b^{2}\right)\right]^{\frac{1}{k+1}}}
\end{aligned}
$$

Segue, imediatamente que:

$$
\begin{aligned}
\tilde{g}(\zeta) & =\zeta^{k} \frac{\left[\left(1-\zeta^{2(k+1)} a^{2}\right)^{k}\left(1-\zeta^{2(k+1)} b^{2}\right)\right]^{\frac{1}{k+1}}}{\left(1-\zeta^{2(k+1)} b^{2}\right)}\left(-(k+1) \zeta^{-(k+2)}\right) d \zeta \\
& =\frac{1}{\zeta^{2}} \frac{-(k+1)\left[\left(1-\zeta^{2(k+1)} a^{2}\right)^{k}\left(1-\zeta^{2(k+1)} b^{2}\right)\right]^{\frac{1}{k+1}}}{\left(1-\zeta^{2(k+1)} b^{2}\right)} d \zeta
\end{aligned}
$$

Em $z=\infty, g$ e $\eta$ têm, respectivamente, um polo de ordem $k$ e um polo de ordem 2. Alem disso, é óbvio que $g$ e $\eta$ são regulares em todo $z \neq e_{i}$ e $\infty$. A tabela abaixo contém resumidamente os calculos feitos acima. 


\begin{tabular}{|c|c|c|c|c|c|c|}
\hline$z$ & $-b$ & $-a$ & 0 & $a$ & $b$ & $\infty$ \\
\hline$g(z)$ & $0^{k}$ & $\infty^{k}$ & $0^{k}$ & $\infty^{k}$ & $0^{k}$ & $\infty^{k}$ \\
\hline$\eta(z)$ & $*$ & $0^{2 k}$ & $*$ & $0^{2 k}$ & $*$ & $\infty^{2}$ \\
\hline $\mathrm{S}$ & $\begin{array}{c}\text { ponto } \\
\text { regular }\end{array}$ & $\begin{array}{c}\text { ponto } \\
\text { regular }\end{array}$ & $\begin{array}{c}\text { ponto } \\
\text { regular }\end{array}$ & $\begin{array}{c}\text { ponto } \\
\text { regular }\end{array}$ & $\begin{array}{c}\text { ponto } \\
\text { regular }\end{array}$ & $\begin{array}{c}\text { fim de ordem } \\
k_{1}=2 k+1\end{array}$ \\
\hline
\end{tabular}

Concluímos que $\eta$ é holomorfa em $\bar{R}_{2 k} \backslash\{\infty\}=R_{2 k}$, e nos pontos em que $g$ tem polos de ordem $k, \eta$ tem zeros de ordem $2 k$, e a condição $\mathbf{c}_{\boldsymbol{1}}$ do teorema 1.5.21 é satisfeita. Observe também que da tabela acima o número de zeros menos o número de polos de $\eta$, contando com a multiplicidade, é $\mathcal{Z}-\mathcal{P}=4 k-2$. Por outro lado, pelo corolário 1.2.9, $\operatorname{gr}[(\eta)]=2 p-2=4 k-2$, e temos uma boa escolha para $\eta$.

Se de fato a superfície $S$ existir, então ela será conformemente equivalente a $\bar{R}_{2 k} \backslash\{\infty\}$, terá gênero $2 k$, um fim em $z\left(P_{1}\right)=\infty$ e $\operatorname{grau}(g)=3 k$, pois pela tabela acima existem três valores distintos que são levados num mesmo ponto $k$ vezes. Assim, a curvatura total será

$$
C(S)=-4 \pi \cdot \operatorname{grau}(g)=-12 k \pi
$$

e pela fórmula dada em 1.5 temos:

$-12 k \pi=2 \pi\left(2-2 p-N-k_{1}\right)=2 \pi\left(2-4 k-1-k_{1}\right)=2 \pi\left(1-4 k-k_{1}\right) \Rightarrow k_{1}=2 k+1$,

que será a ordem do único fim da superfície, caso exista, o qual, obviamente, não será mergulhado.

Daqui para a frente o objetivo é provar que $\phi_{1}, \phi_{2}$ e $\phi_{3}$ não têm períodos reais sobre $\bar{R}_{2 k} \backslash\{\infty\}$. Tais períodos devem ser calculados sobre as curvas fechadas em $\bar{R}_{2 k} \backslash\{\infty\}$ e não triviais na homologia de $\bar{R}_{2 k}$, indicadas na figura 5.1, já que todas as outras curvas fechadas serão combinação linear destas. É imediato verificar que $\operatorname{Re}\left\{\int_{\gamma_{i}^{\prime}} \phi_{3}\right\}=0$, para todo $j=1,2, \ldots, k+1$ e $i=1,2,3,4$.

\section{Demonstração de $c_{2}$ :}


Para demonstrar $\mathbf{c}_{2}$ precisamos somente provar que:

$$
\operatorname{Re}\left\{\int_{\gamma_{i}^{\prime}} \phi_{1}\right\}=\operatorname{Re}\left\{\int_{\gamma_{i}^{j}} \phi_{2}\right\}=0, \quad \forall j=1,2, \ldots, k+1, \quad i=1,2,3,4 .
$$

De 5.4 segue,

$$
\begin{aligned}
\int_{\gamma_{i}^{3}} \phi_{1}(z) & =\frac{1}{2} \int_{\gamma_{i}^{3}} \frac{\left[z\left(z^{2}-a^{2}\right)^{k}\left(z^{2}-b^{2}\right)\right]^{\frac{1}{k+1}}}{z\left(z^{2}-b^{2}\right)} d z-\frac{B^{2}}{2} \int_{\gamma_{i}^{j}} \frac{z\left(z^{2}-b^{2}\right)}{\left[z\left(z^{2}-a^{2}\right)^{k}\left(z^{2}-b^{2}\right)\right]^{\frac{1}{k+1}}} d z \\
\int_{\gamma_{i}^{j}} \phi_{2}(z) & =\frac{i}{2} \int_{\gamma_{i}^{3}} \frac{\left[z\left(z^{2}-a^{2}\right)^{k}\left(z^{2}-b^{2}\right)\right]^{\frac{1}{k+1}}}{z\left(z^{2}-b^{2}\right)} d z+\frac{i B^{2}}{2} \int_{\gamma_{i}^{j}} \frac{z\left(z^{2}-b^{2}\right)}{\left[z\left(z^{2}-a^{2}\right)^{k}\left(z^{2}-b^{2}\right)\right]^{\frac{1}{k+1}}} d z
\end{aligned}
$$

Consideremos $\omega(z)$ o ramo de $w(z)=\left[z\left(z^{2}-a^{2}\right)^{k}\left(z^{2}-b^{2}\right)\right]^{\frac{1}{k+1}}$ definido sobre $\mathbb{C} \backslash\{[-b,-a] \cup[0, a] \cup[b, \infty)\}$ que é por exemplo uma das $j=1,2, \ldots, k+1$ folhas usadas na construção de $\bar{R}_{2 k}$. Suponha que quando $\omega(z)$ esteja na parte superior da linha do corte de cada ramo de $w(z)$, temos $\arg (\omega)=\frac{2 j \pi i}{k+1}$. Por exemplo, na parte superior de $[-b,-a], \omega(z):=e^{\frac{2 \jmath \pi i}{k+1}}\left[x\left(x^{2}-a^{2}\right)\left(x^{2}-b^{2}\right)\right]^{\frac{1}{k+1}}$. Os períodos de $\phi_{1}$ e $\phi_{2}$ sobre as curvas $\gamma_{1}^{j}, \gamma_{2}^{j}, \gamma_{3}^{j}$ e $\gamma_{4}^{j}$, indicadas na figura 5.1, são os mesmos sobre as curvas indicadas na figura 5.4 abaixo.
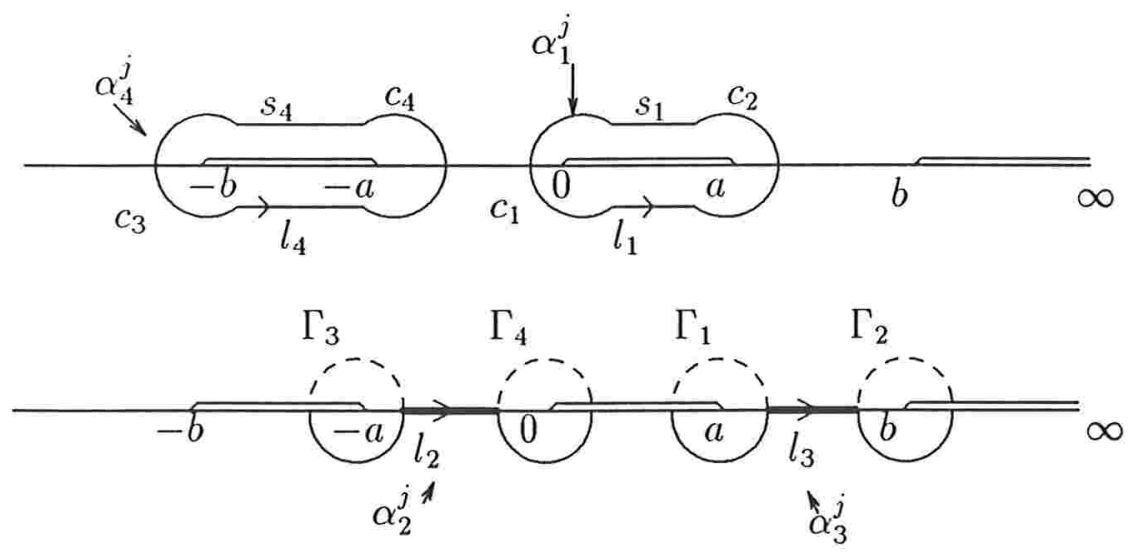

Figura 5.4

Passemos agora aos cálculos dos períodos. O primeiro será, $\int_{\gamma_{1}^{j}} \phi_{1}$ que pela figura 5.4 acima é igual a,

$$
\int_{\gamma_{1}^{3}} \phi_{1}(z)=\int_{\alpha_{1}^{3}} \phi_{1}(z)=\int_{c_{1}} \phi_{1}(z)+\int_{l_{1}} \phi_{1}(x)+\int_{c_{2}} \phi_{1}(z)+\int_{s_{1}} \phi_{1}(x)
$$


Pelos ítens $(i)$ e $($ ii $)$ do lema 3.2.2, temos que $\int_{c_{1}} \phi_{1}=\int_{c_{2}} \phi_{1}=0$, e então:

$$
\int_{\gamma_{1}^{j}} \phi_{1}(z)=\int_{\alpha_{1}^{j}} \phi_{1}(z)=\int_{l_{1}} \phi_{1}(x)+\int_{s_{1}} \phi_{1}(x)
$$

Assim de 5.5, temos

$$
\begin{aligned}
& \int_{\gamma_{1}^{\prime}} \phi_{1}(z)=\frac{1}{2} \int_{0}^{a} \frac{e^{\frac{2(\jmath-1) \pi i}{k+1}}\left[x\left(x^{2}-a^{2}\right)^{k}\left(x^{2}-b^{2}\right)\right]^{\frac{1}{k+1}}}{x\left(x^{2}-b^{2}\right)} d x- \\
& -\frac{B^{2}}{2} \int_{0}^{a} \frac{x\left(x^{2}-b^{2}\right)}{e^{\frac{2(j-1) \pi i}{k+1}}\left[x\left(x^{2}-a^{2}\right)^{k}\left(x^{2}-b^{2}\right)\right]^{\frac{1}{k+1}}} d x+ \\
& +\frac{1}{2} \int_{a}^{0} \frac{e^{\frac{2 j \pi i}{k+1}}\left[x\left(x^{2}-a^{2}\right)^{k}\left(x^{2}-b^{2}\right)\right]^{\frac{1}{k+1}}}{x\left(x^{2}-b^{2}\right)} d x- \\
& -\frac{B^{2}}{2} \int_{a}^{0} \frac{x\left(x^{2}-b^{2}\right)}{e^{\frac{2 j \pi i}{k+1}}\left[x\left(x^{2}-a^{2}\right)^{k}\left(x^{2}-b^{2}\right)\right]^{\frac{1}{k+1}}} d x \\
& \int_{\gamma_{1}^{\prime}} \phi_{1}(z)=\frac{1}{2} e^{\frac{2(\jmath-1) \pi i}{k+1}} \int_{0}^{a} \frac{\left[x\left(a^{2}-x^{2}\right)^{k}\left(b^{2}-x^{2}\right)(-1)^{k+1}\right]^{\frac{1}{k+1}}}{-x\left(b^{2}-x^{2}\right)} d x- \\
& -\frac{1}{2} e^{\frac{2 j \pi i}{k+1}} \int_{0}^{a} \frac{\left[x\left(a^{2}-x^{2}\right)^{k}\left(b^{2}-x^{2}\right)(-1)^{k+1}\right]^{\frac{1}{k+1}}}{-x\left(b^{2}-x^{2}\right)} d x- \\
& -\frac{B^{2}}{2} e^{\frac{-2(j-1) \pi i}{k+1}} \int_{0}^{a} \frac{-x\left(b^{2}-x^{2}\right)}{\left[x\left(a^{2}-x^{2}\right)^{k}\left(b^{2}-x^{2}\right)(-1)^{k+1}\right]^{\frac{1}{k+1}}} d x+ \\
& +\frac{B^{2}}{2} e^{\frac{-2 \jmath \pi i}{k+1}} \int_{0}^{a} \frac{-x\left(b^{2}-x^{2}\right)}{\left[x\left(a^{2}-x^{2}\right)^{k}\left(b^{2}-x^{2}\right)(-1)^{k+1}\right]^{\frac{1}{k+1}}} d x \\
& \int_{\gamma_{1}^{\prime}} \phi_{1}(z)=\frac{1}{2} e^{\frac{2 \jmath \pi i}{k+1}}\left(e^{\frac{-2 \pi i}{k+1}}-1\right) \int_{0}^{a}\left[\frac{a^{2}-x^{2}}{x\left(b^{2}-x^{2}\right)}\right]^{\frac{k}{k+1}} d x- \\
& -\frac{B^{2}}{2} e^{\frac{-2 \jmath \pi i}{k+1}}\left(e^{\frac{2 \pi i}{k+1}}-1\right) \int_{0}^{a}\left[\frac{x\left(b^{2}-x^{2}\right)}{a^{2}-x^{2}}\right]^{\frac{k}{k+1}} d x
\end{aligned}
$$

Pondo: 


$$
\begin{aligned}
F_{1}(a, b)=F_{1} & :=\int_{0}^{a}\left[\frac{a^{2}-x^{2}}{x\left(b^{2}-x^{2}\right)}\right]^{\frac{k}{k+1}} d x \\
G_{1}(a, b)=G_{1} & :=\int_{0}^{a}\left[\frac{x\left(b^{2}-x^{2}\right)}{a^{2}-x^{2}}\right]^{\frac{k}{k+1}} d x \quad \mathrm{e} \\
C_{j} & :=e^{\frac{2 \jmath \pi i}{k+1}}\left(e^{\frac{-2 \pi i}{k+1}}-1\right)
\end{aligned}
$$

E, substituindo acima obtemos,

$$
\int_{\gamma_{1}^{J}} \phi_{1}(z)=\frac{1}{2}\left(C_{j} F_{1}-B^{2} \bar{C}_{j} G_{1}\right)
$$

Agora para calcular

$$
\int_{\gamma_{1}^{3}} \phi_{2}(z)=\int_{\alpha_{1}^{3}} \phi_{2}(z)=\int_{c_{1}} \phi_{2}(z)+\int_{l_{1}} \phi_{2}(x)+\int_{c_{2}} \phi_{2}(z)+\int_{s_{1}} \phi_{2}(x),
$$

de novo usamos os ítens $(i)$ ( $i i)$ do lema 3.2 .2 , nos quais $\int_{c_{1}} \phi_{2}=\int_{c_{2}} \phi_{2}=0$, e a equação 5.7, a menos de sinal e fator de multiplicação. Então:

$$
\int_{\gamma_{1}^{\prime}} o_{2}(z)=\frac{i}{2}\left(C_{j} F_{1}+B^{2} \overline{C_{j}} G_{1}\right)=\frac{1}{2}\left[\left(i C_{j}\right) F_{1}-B^{2}\left(\overline{i C_{j}}\right) G_{1}\right]
$$

Cálculo dos períodos de $\phi_{1}$ e $\phi_{2}$ sobre $\gamma_{2}^{j}$ :

$$
\begin{aligned}
& \int_{\gamma_{2}^{\prime}} \phi_{1}(z)=\int_{\alpha_{2}^{\prime}} \phi_{1}(z)=\int_{\Gamma_{3}} \phi_{1}(z)+\int_{l_{2}} \phi_{1}(x)+\int_{\Gamma_{4}} \phi_{1}(z)+\int_{l_{2}^{-}} \phi_{1}(x) \quad \mathrm{e} \\
& \int_{\gamma_{2}^{\prime}} \phi_{2}(z)=\int_{\alpha_{2}^{J}} \phi_{2}(z)=\int_{\Gamma_{3}} \phi_{2}(z)+\int_{l_{2}} \phi_{2}(x)+\int_{\Gamma_{4}} \phi_{2}(z)+\int_{l_{2}^{-}} \phi_{2}(x)
\end{aligned}
$$

Pelo lema 3.2 .2 ítens (vii) (viii),

$$
\begin{aligned}
& \int_{\gamma_{2}^{\prime}} \phi_{1}(z)=\int_{l_{2}} \phi_{1}(x)+\int_{l_{2}^{-}} \phi_{1}(x)= \\
& =\frac{1}{2} \int_{-a}^{0} \frac{e^{\frac{2(\jmath-1) \pi i}{k+1}}\left[x\left(x^{2}-a^{2}\right)^{k}\left(x^{2}-b^{2}\right)\right]^{\frac{1}{k+1}}}{x\left(x^{2}-b^{2}\right)} d x- \\
& -\frac{B^{2}}{2} \int_{-a}^{0} \frac{x\left(x^{2}-b^{2}\right)}{e^{\frac{2(\jmath-1) \pi i}{k+1}}\left[x\left(x^{2}-a^{2}\right)^{k}\left(x^{2}-b^{2}\right)\right]^{\frac{1}{k+1}}} d x+
\end{aligned}
$$




$$
\begin{aligned}
& +\frac{1}{2} \int_{0}^{-a} \frac{e^{\frac{2 j \pi i}{k+1}}\left[x\left(x^{2}-a^{2}\right)^{k}\left(x^{2}-b^{2}\right)\right]^{\frac{1}{k+1}}}{x\left(x^{2}-b^{2}\right)} d x- \\
& -\frac{B^{2}}{2} \int_{0}^{-a} \frac{x\left(x^{2}-b^{2}\right)}{e^{\frac{2 j \pi i}{k+1}}\left[x\left(x^{2}-a^{2}\right)^{k}\left(x^{2}-b^{2}\right)\right]^{\frac{1}{k+1}}} d x
\end{aligned}
$$

Fazendo a mudança de variável $x=-y$, invertendo os limites de integração, colocando sinais em evidência nos fatores $\left(x^{2}-a^{2}\right)$ e $\left(x^{2}-b^{2}\right)$, com intuito de controlar o sinal da integral, e escrevendo $(-1)^{k+2}=e^{(k+2) \pi i},(-1)^{\frac{(k+2) \pi i}{k+1}}=-e^{\frac{\pi i}{k+1}}$, temos:

$$
\begin{aligned}
\int_{\gamma_{2}^{j}} \phi_{1}(z) & =-\frac{1}{2} e^{\frac{2(j-1) \pi i}{k+1}} e^{\frac{\pi i}{k+1}} \int_{0}^{a} \frac{\left[x\left(a^{2}-x^{2}\right)^{k}\left(b^{2}-x^{2}\right)\right]^{\frac{1}{k+1}}}{x\left(b^{2}-x^{2}\right)} d x+ \\
& +\frac{1}{2} e^{\frac{2 j \pi i}{k+1}} e^{\frac{\pi i}{k+1}} \int_{0}^{a} \frac{\left[x\left(a^{2}-x^{2}\right)^{k}\left(b^{2}-x^{2}\right)\right]^{\frac{1}{k+1}}}{x\left(b^{2}-x^{2}\right)} d x+ \\
& +\frac{B^{2}}{2} e^{\frac{-2(j-1) \pi i}{k+1}} e^{\frac{-\pi i}{k+1}} \int_{0}^{a} \frac{x\left(b^{2}-x^{2}\right)}{\left[x\left(a^{2}-x^{2}\right)^{k}\left(b^{2}-x^{2}\right)\right]^{\frac{1}{k+1}}} d x \\
& -\frac{B^{2}}{2} e^{\frac{-2 j \pi i}{k+1}} e^{\frac{-\pi i}{k+1}} \int_{0}^{a} \frac{x\left(b^{2}-x^{2}\right)}{\left[x\left(a^{2}-x^{2}\right)^{k}\left(b^{2}-x^{2}\right)\right]^{\frac{1}{k+1}}} d x \\
\int_{\gamma_{2}^{3}} \phi_{1}(z) & =-\frac{1}{2} e^{\frac{\pi i}{k+1}}\left[e^{\frac{2 j \pi i}{k+1}}\left(e^{\frac{-2 \pi i}{k+1}}-1\right)\right] \int_{0}^{a}\left[\frac{a^{2}-x^{2}}{x\left(b^{2}-x^{2}\right)}\right]^{\frac{k}{k+1}} d x- \\
& +\frac{B^{2}}{2} e^{\frac{-\pi i}{k+1}}\left[e^{\frac{-2 j \pi i}{k+1}}\left(e^{\frac{2 \pi i}{k+1}}-1\right)\right] \int_{0}^{a}\left[\frac{x\left(b^{2}-x^{2}\right)}{a^{2}-x^{2}}\right]^{\frac{k}{k+1}} d x
\end{aligned}
$$

Portanto,

$$
\int_{\gamma_{2}^{3}} \phi_{1}(z)=-\frac{1}{2}\left[\left(C_{j} e^{\frac{\pi i}{k+1}}\right) F_{1}-B^{2} \overline{\left(C_{j} e^{\frac{\pi i}{k+1}}\right)} G_{1}\right]
$$

Do mesmo modo:

$$
\begin{aligned}
\int_{\gamma_{2}^{\prime}} \phi_{2}(z) & =-\frac{i}{2}\left[\left(C_{j} e^{\frac{\pi i}{k+1}}\right) F_{1}+B^{2} \overline{\left(C_{j} e^{\frac{\pi i}{k+1}}\right)} G_{1}\right] \\
\int_{\gamma_{2}^{\prime}} \phi_{2}(z) & =-\frac{1}{2}\left[\left(i C_{j} e^{\frac{\pi i}{k+1}}\right) F_{1}-B^{2} \overline{\left(i C_{j} e^{\frac{\pi i}{k+1}}\right)} G_{1}\right]
\end{aligned}
$$

Os períodos de $\phi_{1}$ e $\phi_{2}$ sobre $\gamma_{3}^{j}$, usando os ítens $(v)$ (vi) do lema 3.2.2 são:

$$
\int_{\gamma_{3}^{3}} \phi_{1}(z)=\int_{l_{3}} \phi_{1}(x)+\int_{l_{3}^{-}} \phi_{1}(x) \quad \text { e } \int_{\gamma_{3}^{j}} \phi_{2}(z)=\int_{l_{3}} \phi_{2}(x)+\int_{l_{3}^{-}} \phi_{2}(x)
$$


Então,

$$
\begin{aligned}
\int_{\gamma_{3}^{3}} \phi_{1}(z) & =\frac{1}{2} \int_{a}^{b} \frac{e^{\frac{2(\jmath-1) \pi i}{k+1}}\left[x\left(x^{2}-a^{2}\right)^{k}\left(x^{2}-b^{2}\right)\right]^{\frac{1}{k+1}}}{x\left(x^{2}-b^{2}\right)} d x- \\
& -\frac{B^{2}}{2} \int_{a}^{b} \frac{x\left(x^{2}-b^{2}\right)}{e^{\frac{2(\jmath-1) \pi i}{k+1}}\left[x\left(x^{2}-a^{2}\right)^{k}\left(x^{2}-b^{2}\right)\right]^{\frac{1}{k+1}}} d x+ \\
& +\frac{1}{2} \int_{b}^{a} \frac{e^{\frac{2 j \pi i}{k+1}}\left[x\left(x^{2}-a^{2}\right)^{k}\left(x^{2}-b^{2}\right)\right]^{\frac{1}{k+1}}}{x\left(x^{2}-b^{2}\right)} d x- \\
& -\frac{B^{2}}{2} \int_{b}^{a} \frac{x\left(x^{2}-b^{2}\right)}{e^{\frac{2 \jmath \pi t}{k+1}}\left[x\left(x^{2}-a^{2}\right)^{k}\left(x^{2}-b^{2}\right)\right]^{\frac{1}{k+1}}} d x
\end{aligned}
$$

Por um cálculo análogo ao feito em $\int_{\gamma_{2}^{\prime}} \phi_{1}$, temos:

$$
\begin{aligned}
\int_{\gamma_{3}^{3}} \phi_{1}(z) & =-\frac{1}{2} e^{\frac{\pi i}{k+1}}\left[e^{\frac{2 \jmath \pi i}{k+1}}\left(e^{\frac{-2 \pi i}{k+1}}-1\right)\right] \int_{a}^{b}\left[\frac{x^{2}-a^{2}}{x\left(b^{2}-x^{2}\right)}\right]^{\frac{k}{k+1}} d x+ \\
& +\frac{B^{2}}{2} e^{\frac{-\pi i}{k+1}}\left[e^{\frac{-23 \pi i}{k+1}}\left(e^{\frac{2 \pi i}{k+1}}-1\right)\right] \int_{a}^{b}\left[\frac{x\left(b^{2}-x^{2}\right)}{x^{2}-a^{2}}\right]^{\frac{k}{k+1}} d x
\end{aligned}
$$

Logo:

$$
\begin{aligned}
& \int_{\gamma_{3}^{\prime}} \phi_{1}(z)=-\frac{1}{2}\left[\left(C_{j} e^{\frac{\pi i}{k+1}}\right) F_{2}-B^{2} \overline{\left(C_{j} e^{\frac{\pi i}{k+1}}\right)} G_{2}\right] \mathrm{e} \\
& \int_{\gamma_{3}^{\prime}} \phi_{2}(z)=-\frac{1}{2}\left[\left(i C_{j} e^{\frac{\pi i}{k+1}}\right) F_{2}-B^{2} \overline{\left(i C_{j} e^{\frac{\pi i}{k+1}}\right)} G_{2}\right]
\end{aligned}
$$

onde,

$$
\begin{aligned}
F_{2}(a, b)=F_{2} & =\int_{a}^{b}\left[\frac{x^{2}-a^{2}}{x\left(b^{2}-x^{2}\right)}\right]^{\frac{k}{k+1}} d x \quad \mathrm{e} \\
G_{2}(a, b)=G_{2} & =\int_{a}^{b}\left[\frac{x\left(b^{2}-x^{2}\right)}{x^{2}-a^{2}}\right]^{\frac{k}{k+1}} d x
\end{aligned}
$$

são constantes reais positivas. Finalmente,

$$
\int_{\gamma_{4}^{3}} \phi_{1}(z)=\int_{l_{4}} \phi_{1}(x)+\int_{s_{4}} \phi_{1}(x) \quad \text { e } \int_{\gamma_{4}^{3}} \phi_{2}(z)=\int_{l_{4}} \phi_{2}(x)+\int_{s_{4}} \phi_{2}(x)
$$


Logo,

$$
\begin{aligned}
\int_{\gamma_{4}^{\prime}} \phi_{1}(z) & =\frac{1}{2} \int_{-b}^{-a} \frac{e^{\frac{2(j-1) \pi i}{k+1}}\left[x\left(x^{2}-a^{2}\right)^{k}\left(x^{2}-b^{2}\right)\right]^{\frac{1}{k+1}}}{x\left(x^{2}-b^{2}\right)} d x- \\
& -\frac{B^{2}}{2} \int_{-b}^{-a} \frac{x\left(x^{2}-b^{2}\right)}{e^{\frac{2(j-1) \pi i}{k+1}}\left[x\left(x^{2}-a^{2}\right)^{k}\left(x^{2}-b^{2}\right)\right]^{\frac{1}{k+1}}} d x+ \\
& +\frac{1}{2} \int_{-a}^{-b} \frac{e^{\frac{2 j \pi i}{k+1}}\left[x\left(x^{2}-a^{2}\right)^{k}\left(x^{2}-b^{2}\right)\right]^{\frac{1}{k+1}}}{x\left(x^{2}-b^{2}\right)} d x- \\
& -\frac{B^{2}}{2} \int_{-a}^{-b} \frac{x\left(x^{2}-b^{2}\right)}{e^{\frac{2 j \pi i}{k+1}}\left[x\left(x^{2}-a^{2}\right)^{k}\left(x^{2}-b^{2}\right)\right]^{\frac{1}{k+1}}} d x
\end{aligned}
$$

Donde vem,

$$
\begin{aligned}
\int_{\gamma_{4}^{\prime}} \phi_{1}(z) & =\frac{1}{2} e^{\frac{2 j \pi i}{k+1}}\left(e^{\frac{-2 \pi i}{k+1}}-1\right) \int_{a}^{b}\left[\frac{x^{2}-a^{2}}{x\left(b^{2}-x^{2}\right)}\right]^{\frac{k}{k+1}} d x- \\
& -\frac{B^{2}}{2} e^{\frac{-2 \jmath \pi i}{k+1}}\left(e^{\frac{2 \pi i}{k+1}}-1\right) \int_{a}^{b}\left[\frac{x\left(b^{2}-x^{2}\right)}{x^{2}-a^{2}}\right]^{\frac{k}{k+1}} d x .
\end{aligned}
$$

\begin{tabular}{|c|c|c|}
\hline curvas & $\gamma_{3}^{j}$ & $\gamma_{4}^{j}$ \\
\hline $\int \phi_{1}$ & $-\frac{1}{2}\left[\left(C_{j} e^{\frac{\pi i}{k+1}}\right) F_{2}-B^{2}\left(C_{j} e^{\frac{\pi 1}{k+1}}\right) G_{2}\right]$ & $\frac{1}{2}\left(C_{j} F_{2}-B^{2} \overline{C_{j}} G_{2}\right)$ \\
\hline $\int \phi_{2}$ & $-\frac{1}{2}\left[\left(i C_{j} e^{\frac{\pi i}{k+1}}\right) F_{2}-B^{2}\left(i C_{j} e^{\frac{\pi i}{k+1}}\right) G_{2}\right]$ & $\frac{1}{2}\left(i C_{j} F_{2}-B^{2}\left(\overline{i C_{j}}\right) G_{2}\right)$ \\
\hline
\end{tabular}

Portanto,

$$
\begin{aligned}
\int_{\gamma_{4}^{\prime}} \phi_{1}(z) & =\frac{1}{2}\left(C_{j} F_{2}-B^{2} \overline{C_{j}} G_{2}\right) \\
\int_{\gamma_{4}^{3}} \phi_{2}(z) & =\frac{1}{2}\left[\left(i C_{j}\right) F_{2}-B^{2} \overline{\left(i C_{j}\right)} G_{2}\right]
\end{aligned}
$$

Reunindo nas tabelas abaixo todos os períodos obtemos,

\begin{tabular}{|c|c|c|}
\hline curvas & $\gamma_{1}^{j}$ & $\gamma_{2}^{j}$ \\
\hline $\int \phi_{1}$ & $\frac{1}{2}\left(C_{j} F_{1}-B^{2} \overline{C_{j}} G_{1}\right)$ & $-\frac{1}{2}\left[\left(C_{j} e^{\frac{\pi i}{k+1}}\right) F_{1}-B^{2}\left(\overline{C_{j} e^{\frac{\pi i}{k+1}}}\right) G_{1}\right]$ \\
\hline $\int \phi_{2}$ & $\frac{1}{2}\left(\left(i C_{j}\right) F_{1}-B^{2}\left(\overline{i C_{j}}\right) G_{1}\right)$ & $-\frac{1}{2}\left[\left(i C_{j} e^{\frac{\pi i}{k+1}}\right) F_{1}-B^{2}\left(i C_{j} e^{\frac{\pi i}{k+1}}\right) G_{1}\right]$ \\
\hline
\end{tabular}


Precisamos determinar uma única condição, a fim de que todas as partes reais dos períodos das tabelas acima se anulem. Neste sentido,

$$
\begin{aligned}
& \operatorname{Re}\left\{\int_{\gamma_{1}^{\prime}} \phi_{1}\right\}=\frac{1}{4}\left(C_{j}+\overline{C_{j}}\right)\left(F_{1}-B^{2} G_{1}\right), \quad \text { e então, } \\
& \operatorname{Re}\left\{\int_{\gamma_{1}^{3}} \phi_{1}\right\}=0 \quad \Leftrightarrow \quad C_{j}+\overline{C_{j}}=0 \quad \text { ou } \quad F_{1}-B^{2} G_{1}=0
\end{aligned}
$$

Caso $C_{j}+\overline{C_{j}}=0, \quad \forall j$, então $\operatorname{Re}\left\{C_{j}\right\}=0$, logo $C_{j}$ é puramente imaginário. Das tabelas acima é fácil concluir que:

$$
\begin{gathered}
\operatorname{Re}\left\{\int_{\gamma_{1}^{\prime}} \phi_{1}\right\}=0, \quad \text { para todo } \mathrm{j}=1,2, \ldots, k+1 \text { e somente } i=1,4 . \\
\operatorname{Re}\left\{\int_{\gamma_{1}^{\prime}} \phi_{2}\right\}=\frac{1}{4}\left(\left(i C_{j}\right)+\overline{\left(i C_{j}\right)}\right)\left(F_{1}-B^{2} G_{1}\right) .
\end{gathered}
$$

Logo:

$$
\operatorname{Re}\left\{\int_{\gamma_{1}^{\prime}} \phi_{2}\right\}=0 \quad \Leftrightarrow \quad\left(i C_{j}\right)+\overline{\left(i C_{j}\right)}=0 \quad \text { ou } \quad F_{1}-B^{2} G_{1}=0
$$

Se $\left(i C_{j}\right)+\overline{\left(i C_{j}\right)}=0, \quad \forall j, \quad \operatorname{Re}\left\{i C_{j}\right\}=-\operatorname{Im}\left\{C_{j}\right\}=0$. Suponhamos que $F_{1}-B^{2} G_{1} \neq 0$, então $\operatorname{Re}\left\{\int_{\gamma_{1}^{\prime}} \phi_{1}\right\}=\operatorname{Re}\left\{\int_{\gamma_{1}^{\prime}} \phi_{2}\right\}=0$, somente se, respectivamente, $\operatorname{Re}\left\{C_{j}\right\}=\operatorname{Im}\left\{C_{j}\right\}=0$, isto é, $C_{j}=0$, para todo $j=1,2, \ldots, k+1$. O que é uma contradição, isto é, não existe uma única condição para $C_{j}$, de modo que

$$
\operatorname{Re}\left\{\int_{\gamma_{1}^{3}} \phi_{1}\right\}=\operatorname{Re}\left\{\int_{\gamma_{1}^{\prime}} \phi_{2}\right\}=0 \forall j=1,2, \ldots, k+1 \text { e } i=1,2,3,4 .
$$

Calculando as outras partes reais dos periodos restantes chegamos a:

$$
\begin{aligned}
& \operatorname{Re}\left\{\int_{\gamma_{2}^{\prime}} \phi_{1}\right\}=-\frac{1}{4}\left(\left(C_{j} e^{\frac{\pi i}{k+1}}\right)+\overline{\left(C_{j} e^{\frac{\pi i}{k+1}}\right)}\right)\left(F_{1}-B^{2} G_{1}\right) \\
& \operatorname{Re}\left\{\int_{\gamma_{2}^{\prime}} \phi_{2}\right\}=-\frac{1}{4}\left(\left(i C_{j} e^{\frac{\pi i}{k+1}}\right)+\overline{\left(i C_{j} e^{\frac{\pi i}{k+1}}\right)}\right)\left(F_{1}-B^{2} G_{1}\right)
\end{aligned}
$$




$$
\begin{aligned}
& \operatorname{Re}\left\{\int_{\gamma_{3}^{J}} \phi_{1}\right\}=\frac{1}{4}\left(C_{j}+\overline{C_{j}}\right)\left(F_{2}-B^{2} G_{2}\right) \\
& \operatorname{Re}\left\{\int_{\gamma_{3}^{J}} \phi_{2}\right\}=\frac{1}{4}\left(\left(i C_{j}\right)+\overline{\left(i C_{j}\right)}\right)\left(F_{2}-B^{2} G_{2}\right) \\
& \operatorname{Re}\left\{\int_{\gamma_{4}^{J}} \phi_{1}\right\}=-\frac{1}{4}\left(\left(C_{j} e^{\frac{\pi i}{k+1}}\right)+\overline{\left(C_{j} e^{\frac{\pi i}{k+1}}\right)}\right)\left(F_{2}-B^{2} G_{2}\right) \\
& \operatorname{Re}\left\{\int_{\gamma_{4}^{J}} \phi_{2}\right\}=-\frac{1}{4}\left(\left(i C_{j} e^{\frac{\pi i}{k+1}}\right)+\overline{\left(i C_{j} e^{\frac{\pi i}{k+1}}\right)}\right)\left(F_{2}-B^{2} G_{2}\right)
\end{aligned}
$$

Assim, para que os períodos reais sejam nulos, temos que satisfazer às condições:

$$
F_{1}-B^{2} G_{1}=0 \quad \text { e } \quad F_{2}-B^{2} G_{2}=0, \quad \text { ou equivalentemente, } B^{2}=\frac{F_{1}}{G_{1}}=\frac{F_{2}}{G_{2}}
$$

Para concluir que vale $c_{2}$, o problema se reduz em provar que existem constantes reais $a, b$ e $B, \operatorname{com} 0<a<b, 0<B$ tal que valha 5.9.

As integrais $F_{m}$ e $G_{m}, \quad m=1,2$ definidas em 5.6 e 5.8 são constantes reais positivas. Por exemplo, mostremos que: $0<F_{1}<\infty$.

De fato,

$$
\frac{a-x}{b(b+a)} \leq \frac{a^{2}-x^{2}}{x\left(b^{2}-x^{2}\right)} \leq \frac{2 a^{2}}{b(b-a) x}
$$

Assim,

$$
0<\frac{k+1}{2 k+1}\left[\frac{1}{b(b+a)}\right]^{\frac{k}{k+1}} a^{\frac{2 k+1}{k+1}} \leq F_{1} \leq(k+1)\left[\frac{2 a^{2}}{b(b-a)}\right]^{\frac{k}{k+1}} a^{\frac{1}{k+1}}
$$

Lema 5.0.8 Sejam $F_{m}$ e $G_{m} \quad m=1,2$ constantes reais positivas definidas em 5.6 5.8. Para qualquer $k \in \mathbb{Z}_{+}$, existem constantes reais $a, b$ e $B$ satisfazendo, $0<a<b, 0<B$, tais que $B^{2}=\frac{F_{1}}{G_{1}}=\frac{F_{2}}{G_{2}}$.

\section{Demonstração:}

Fixados $a$ e $b$, escolhemos $B=\sqrt{\frac{F_{1}}{G_{1}}}$. Além disso, através de uma transformação de Möbius podemos supor $a=1$. Logo, $F_{m}$ e $G_{m}$ passam a depender somente de $b>1$. Daí, o lema ficará provado se existir $b$ tal que

$$
F_{1}(1, b) G_{2}(1, b)=F_{2}(1, b) G_{1}(1, b)
$$


Agora de 5.6 e 5.8 temos,

$$
\begin{aligned}
& F_{1} G_{2}=\int_{0}^{1}\left[\frac{1-x^{2}}{x\left(b^{2}-x^{2}\right)}\right]^{\frac{k}{k+1}} d x \int_{1}^{b}\left[\frac{x\left(b^{2}-x^{2}\right)}{x^{2}-1}\right]^{\frac{k}{k+1}} d x \\
& F_{2} G_{1}=\int_{1}^{b}\left[\frac{x^{2}-1}{x\left(b^{2}-x^{2}\right)}\right]^{\frac{k}{k+1}} d x \int_{0}^{1}\left[\frac{x\left(b^{2}-x^{2}\right)}{1-x^{2}}\right]^{\frac{k}{k+1}} d x
\end{aligned}
$$

Fazendo a mudança de variável $y=x-1$ em $F_{2}$ e $G_{2}$ obtemos,

$$
\begin{aligned}
& F_{2}=\int_{0}^{b-1}\left[\frac{y(y+2)}{(y+1)\left(b^{2}-(y+1)^{2}\right)}\right]^{\frac{k}{k+1}} d y \\
& G_{2}=\int_{0}^{b-1}\left[\frac{(y+1)\left(b^{2}-(y+1)^{2}\right)}{(y+2) y}\right]^{\frac{k}{k+1}} d y
\end{aligned}
$$

Assim, de 5.10, segue

$$
\begin{aligned}
F_{1} G_{2}= & \int_{0}^{1}\left[\frac{1+x}{(b+x)(b-x)}\right]^{\frac{k}{k+1}}\left[\frac{1-x}{x}\right]^{\frac{k}{k+1}} d x \\
& \cdot \int_{0}^{b-1}\left[\frac{(x+1)(b+x+1)}{x+2}\right]^{\frac{k}{k+1}}\left[\frac{(b-x-1)}{x}\right]^{\frac{k}{k+1}} d x \\
F_{2} G_{1}= & \int_{0}^{b-1}\left[\frac{(x+2) x}{(x+1)\left(b^{2}-(x+1)^{2}\right)}\right]^{\frac{k}{k+1}} d x \int_{0}^{1}\left[\frac{x\left(b^{2}-x^{2}\right)}{1-x^{2}}\right]^{\frac{k}{k+1}} d x
\end{aligned}
$$

Agora fazendo, mais duas mudanças de variáveis $y=x-1$ e $y=x-(b-1)$, respectivamente, nas integrais

$$
\int_{0}^{1}\left[\frac{x\left(b^{2}-x^{2}\right)}{1-x^{2}}\right]^{\frac{k}{k+1}} d x \quad \text { e } \int_{0}^{b-1}\left[\frac{(x+2) x}{(x+1)\left(b^{2}-(x+1)^{2}\right)}\right]^{\frac{k}{k+1}} d x
$$

E, substituindo na segunda equação de 5.11 obtemos,

$$
\begin{aligned}
F_{2} G_{1}= & \int_{0}^{1}\left[\frac{(b-1+x)(b+1-x)}{2-x}\right]^{\frac{k}{k+1}}\left[\frac{1-x}{x}\right]^{\frac{k}{k+1}} d x \\
& \cdot \int_{0}^{b-1}\left[\frac{b+1-x}{(b-x)(2 b-x)}\right]^{\frac{k}{k+1}}\left[\frac{b-1-x}{x}\right]^{\frac{k}{k+1}} d x
\end{aligned}
$$

Pelos ítens (i) (iii) do lema 3.2.3, substituídos na primeira equação de 5.11, segue-se,

$$
F_{1} G_{2} \leq\left(\frac{4}{b-1}\right)^{\frac{k}{k+1}}\left\{\int_{0}^{1}\left[\frac{1-x}{x}\right]^{\frac{k}{k+1}} d x \int_{0}^{b-1}\left[\frac{b-1-x}{x}\right]^{\frac{k}{k+1}} d x\right\}
$$


Ainda pelo lema 3.2.3 ítens (ii) e $(i v)$ substituídos na equação 5.12 temos,

$$
F_{2} G_{1} \geq\left(\frac{b-1}{4}\right)^{\frac{k}{k+1}}\left\{\int_{0}^{1}\left[\frac{1-x}{x}\right]^{\frac{k}{k+1}} d x \int_{0}^{b-1}\left[\frac{b-1-x}{x}\right]^{\frac{k}{k+1}} d x\right\}
$$

Portanto, de 5.13 e 5.14 e para $b \longrightarrow \infty$ obtemos,

$$
F_{1} G_{2} \leq F_{2} G_{1}
$$

Por outro lado, se $b \longrightarrow 1$, e pondo $b=1+\varepsilon, \varepsilon>0$. Segue-se do lema 3.2 .4 ítens (i) e (iii) e da primeira equação de 5.11 ,

$$
F_{1} G_{2} \geq \int_{0}^{1}\left[\frac{0,81}{1+\varepsilon-x}\right]^{\frac{k}{k+1}}\left[\frac{1-x}{x}\right]^{\frac{k}{k+1}} d x \int_{0}^{\varepsilon}\left[\frac{\varepsilon-x}{x}\right]^{\frac{k}{k+1}} d x
$$

Em em 5.12 se usarmos os ítens (ii) (iv) do lema 3.2.4 resulta,

$$
F_{1} G_{2} \leq \int_{0}^{1}[(0,21)(\varepsilon+x)]^{\frac{k}{k+1}}\left[\frac{1-x}{x}\right]^{\frac{k}{k+1}} d x \int_{0}^{\varepsilon}\left[\frac{\varepsilon-x}{x}\right]^{\frac{k}{k+1}} d x
$$

$\mathrm{O}$ ítem $(v)$ do lema 3.2.4 junto com as inequações 5.16 e 5.17 implica que:

$$
F_{1} G_{2} \geq F_{2} G_{1}
$$

Portanto, de 5.15, 5.18 e o teorema do valor intermediário, existe pelo menos um $b \in(1, \infty)$ tal que vale a equação $F_{1} G_{2}=F_{2} G_{1}$ é válida.

Voltando a demonstração do teorema, concluímos que a partir do lema acima não só resolvemos o problema dos períodos como também determinamos o valor de $B$, $B=\sqrt{\frac{F_{1}}{G_{1}}}$.

\section{Demonstração de $c_{3}$ :}

Como a superfície $S$ só tem um fim, no ponto $z\left(P_{1}\right)=\infty$, então o problema local dos períodos equivale a calcular o resíduo de $\phi_{1}, \phi_{2}$ e $\phi_{3}$ em torno de $P_{1}$. Mas, da tabela dos períodos é fácil concluir que nesse ponto $\phi_{1}$ e $\phi_{2}$ têm polos de ordem $2 k+2$. Logo, $\operatorname{Res}\left(\phi_{1} ; \infty\right)=\operatorname{Res}\left(\phi_{2} ; \infty\right)=\operatorname{Res}\left(\phi_{3} ; \infty\right)=0$. Isto prova a condição $\mathbf{c}_{3}$. 


\section{Demonstração de $\mathrm{c}_{4}$ :}

De fato. pondo $\zeta=1 / z$, para verificar que a superfície é completa em termos da métrica $d s^{2}=\lambda^{2}|d \zeta|^{2}$, é suficiente analizar o comportamento da curva $\gamma$ divergente para $\zeta=0$. Sabemos que $(g, \eta)$ têm, respectivamente, polo de ordem $k$ e 2 em $z=\infty$. Logo, é fácil provar que:

$d s=\lambda(\zeta)|d \zeta| \geq \frac{M}{|\zeta|^{2 k+2}}|d \zeta|$ e portanto, $\operatorname{comp}(\gamma)=\int_{\gamma} d s \geq M\left|\lim _{\zeta \rightarrow 0} \int \frac{1}{\zeta^{2 k+2}} d \zeta\right| \longrightarrow \infty$.

O que conclue a demonstração do teorema.

Quando $k=2$ no teorema 5.0.7, conforme figuras 5.2 e 5.3, o gráfico da superfície mínima em $\mathbb{R}^{3}$ de gênero 4 , curvatural total $-24 \pi$ e um único fim do tipo Enneper é dado como em [21] por:

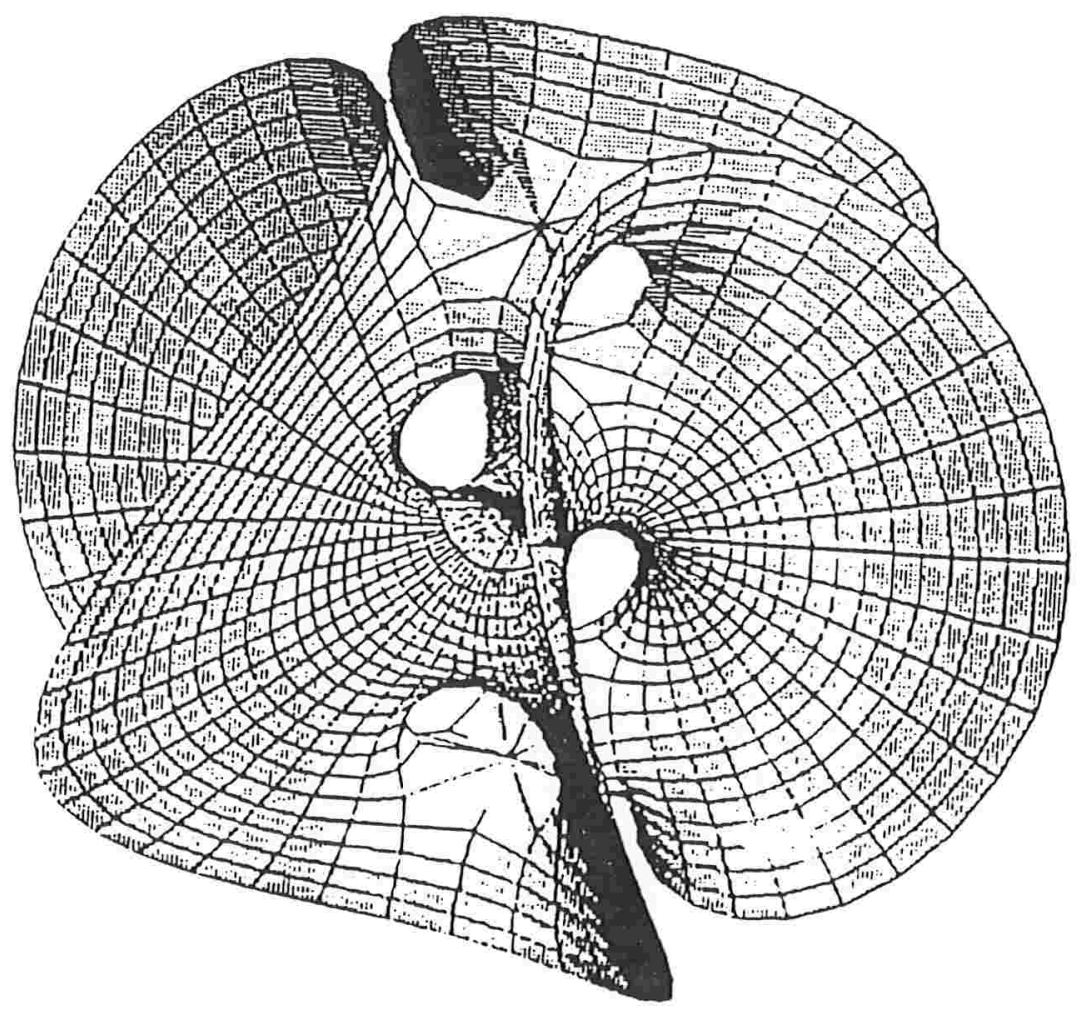




\section{Referências Bibliográficas}

[1] Chen, C.C., Gackstäter, F.: Elliptische und Hyperellitische Funktionen und Vollständige Minimalflächen vom Enneperschen Typ. Math.Ann. 250; 359-369, 1982.

[2] Conway, J.B.: Complex Analysis. Springer-Verlag

[3] Costa, C..J.: Imersões mínimas completas em $\mathbb{R}^{3}$ de gênero um e curvatura total finita. Tese de Doutorado, IMPA, 1982.

[4] Costa, C..J.: Funções Elípticas, Algébricas e Superfícies Mínimas. 18o Colóquio Brasileiro de Matemática, IMPA, 1991.

[5] Dierkes, U., Hildebrandt, S., Küster, A., Wohlrab, O.: Minimal Surfaces I. Springer-Verlag, 1992.

[6] Espírito Santo, N.: Superfícies mínimas completas em $\mathbb{R}^{3}$ com fim do tipo Enneper. Tese de Doutorado, IMPA, 1993.

[7] Farkas, H.M., Kra, Irwin.: Riemann Surfaces. Springer-Verlag, 1980.

[8] Gackstäter, F.: Über Abelsche Minimalfächen. Math. Nachr. 74, 157-165, 1974.

[9] Galvão, M.E.E.L.: Superfícies Mínimas. Programa de Verão, UFBA (versão preliminar). 
[10] Gray, A.: Modern Differential Geometry of Curves and Surfaces with Mathematica. CRC Press, $2^{\text {nd }}$ edition, 1998.

[11] Hoffman, D., Karcher, H.: Complete Embedded Minimal Surfaces of Finite Total Cuvature

[12] Hoffman, D., MeeksIII, W.H.: A complete embedded minimal surface in $\mathbb{R}^{3}$ with genus one and three ends. J. Differential Geom., 21, 109-127, 1985.

[13] Jorge, L.P., Meeks III, W.H.: The topology of complete surfaces of finite total Gaussian curvature. Topology 22, num 2, 203-221, 1983.

[14] Jost, J. : Compact Riemann Surfaces: an introduction to contemporary mathematics. Springer-Verlag, 1997.

[15] Lima, E.L.: Curso de Análise, vol 1. Projeto Euclides, IMPA, 1976.

[16] López, F..J.: The classification os complete minimal surfaces with total curvature greater than $-12 \pi$. Trans. AMS 334, num 1, 49-74, 1992.

[17] López, F.J., Martín, F., Rofríguez, D.: Complete minimal surfaces derived from Chen-Gackstätter genus two example. Pacific Journal of mathematics, 184, No. $2,1998$.

[18] Nevanlinna R., Paatero V.: Introduction to Complex Analysis. Addison-Wesley Publishing Company, 1969.

[19] Osserman, R.: A survey of Minimal Surfaces. Dover Publications, $2^{\text {nd }}$ edition, 1986.

[20] Rodrigues, A.M.: Teoria das Superfícies de Riemann. Faculdade de Filosofia da U. de São Paulo, 1957-1958.

[21] Sato, K.: Construction of higher genus complete minimal surfaces with one end and finite total curvature. titech-math 24, 15-93, 1993. 
[22] Schoen, M. R. : Uniqueness, symmetry, and embeddedness of minimal surfaces. D. Geometry., 18, 791-809, 1983.

[23] Spivak, M. : Comprehensive introduction to differential geometry. Boston, Publish or Perish, 1975, vol. 4.

[24] Springer, G.: Introduction to Riemann Surfaces. Addison-Wesley, 1957.

[25] Thayer, E. C.: Higher genus Chen-Gackstätter surfaces and the Weierstrass representation for infinite genus surfaces. to appear in Jour. of Experimental Mathematics, 4(1) (1995), 11-31.

[26] IVang, H., Kang, J.: A family of complete immersed minimal surfaces with only one end. IMPA, 1991. 


\section{Índice Remissivo}

$L$-equivalente, 6

$\mathbb{C} \cup\{\infty\}, 5$

estrutura

diferenciável, 4

holomorfa. 4

gênero, 50

ordem de ramificação, 9

ponto de ramificação, 9

atlas, 4

biholomorfa, 8

bijeção conforme, 20

comprimento infinito, 67

coordenadas isotérmicas, 40

curva

divergente, 67

divisor, 11

elemento de função, 19

equação de $\wp^{\prime}, 36$

fórmula

da adição, 55

de Riemann, 16
Jorge-Meeks-Gackstätter, 58

fórmulas

de Weierstrass, 62

figura modular, 7

fim, 50

FM, 58

função

$\wp$ de Weierstrass, 31

$\zeta$ de Weierstrass, 31

algébrica, 19

elíptica, 30

grau de $f, 9$

hiperelíptica, 17

multiplicade, 9

multiplicidade $k_{\nu}, 50$

período

primeiro, 64

segundo, 65

quadrado, 53

relação de Legendre, 33

reticulado, 5 
superfície, 41

$$
\begin{aligned}
& \operatorname{com} C(S)=-16 \pi, 115 \\
& \operatorname{com} C(S)=-24 \pi, 133 \\
& \text { de Riemann, } 4 \\
& \text { hiperelíptica, } 28 \\
& \text { mínima, } 42 \\
& \text { mínima completa, } 49
\end{aligned}
$$

teorema

Chen-Gackstätter 1, 60

Chen-Gackstätter 2, 72

H. Wang-J.Kang 2, 118

H. Wang-J.Kang 1, 102

Huber-Osserman, 51

Riemann-Roch, 13

variedade diferenciável, 3 